\title{
Identificação de parâmetros em problemas de advecção-difusão combinando a técnica do operador adjunto e métodos de volumes finitos de alta ordem
}

\author{
Alessandro Alves Santana
}

TESE APRESENTADA

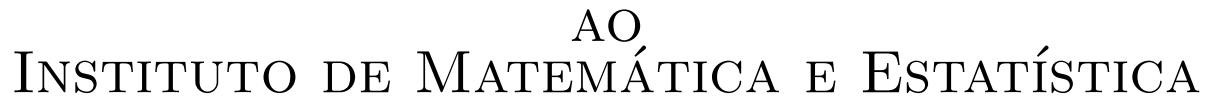

DA

Universidade de São Paulo

PARA

OBTENÇÃA DO TÍTULO

$\mathrm{DE}$

Doutor EM CiênCIAS

Área de Concentração: Matemática Aplicada

Orientador: Prof. Dr. Luis Carlos de Castro Santos

Durante o desenvolvimento deste trabalho o autor recebeu auxílio financeiro da CAPES

São Paulo, novembro de 2007 


\section{Agradecimentos}

Durante o desenvolvimento desse trabalho aconteceram muitas coisas em minha vida. Com muita luta cheguei ao final do presente texto, que é o resultado de tudo o que foi estudado e desenvolvido. O doutorado é o verdadeiro início do pesquisador e o desenvolvimento da ciência é um trabalho infinito.

Agradeço ao meu orientador, professor Dr. Luis Carlos Castro Santos, por toda ajuda, companheirismo e amizade durante a execução desse trabalho. Adquiri com ele uma bagagem grande de conhecimento dentro da computação científica, a qual irei utilizar em toda minha carreira acadêmica.

Ao IME-USP, Instituto de Matemática e Estatística da Universidade de São Paulo, agradeço por ter me permitido participar do programa de doutorado.

Houve uma parte em comum, dentro de dois projetos de doutorado distintos, dirigidos pelo meu orientador, que exigia o estudo e implementação de um método numérico, muito pouco conhecido no Brasil e relativamente recente na literatura, utilizado na resolução de equações diferenciais parciais, o qual é apresentado nessa tese. Assim sendo, esse solver que foi criado é o resultado de um esforço conjunto entre eu e Olga Harumi Saito, a qual agradeço por toda cooperação.

Agradeço aos professores do IME-USP Dr. Saulo Rabello Maciel de Barros e Dr. Alexandre Megiorin Roma. O primeiro pela ajuda ao permitir a utilização de seu computador. O segundo pela ajuda ao permitir a utilização de seus dois servidores obtidos junto a FAPESP em um projeto de pesquisa - processo número 04/13781-1.

À CAPES agradeço pela bolsa de estudos.

Agradeço ao LCCA-USP, Laboratório de Computação Científica Avançada da Universidade de São Paulo, agradeço por permitir a utilização se seus processadores. Ao ICMC-USP, Instituto de Ciências Matemáticas e de Computação da Universidade de São Paulo - Campus de São Carlos, agradeço ao professor Dr. José Alberto Cuminato, o qual foi meu orientador no mestrado, por permitir a utilização do LCAD, Laboratório de Computação de Alto Desempenho, durante o período do doutorado em que precisei residir em São Carlos 
por motivos de problemas em família.

Agradeço a todos os funcionários do IME-USP pelos atendimentos. Agradeço aos meus amigos do IMEUSP pelo companherismo.

Agradeço a direção da FAMAT-UFU, Faculdade de Matemática da Universidade Federal de Uberlândia, instituição na qual atualmente sou professor, pela redução de carga horária para que pudesse ter mais tempo para me dedicar ao doutorado.

À Teodora Pinheiro Figueroa, minha esposa, agradeço pela paciência, tolerância e força durante o doutorado. A José Alves da Silva, meu pai, Marco Túlio Santana Alves, meu irmão, e Charmenie Santana Alves, minha irmã, agradeço por toda vibração positiva nos momentos difícieis. A Meigna Santana da Silva, minha mãe, que embora já não esteja mais entre a gente, a qual agradeço por muito do que sou hoje, tenho certeza que lá de cima ficou torcendo por mim.

Finalmente agradeço a Deus, por me permitir a oportunidade de poder estudar e chegar aonde estou hoje. Por ter me dado forças e ter extendido a mão em todos os momentos de minha vida em que mais precisava de apoio. 


\section{Resumo}

Esse trabalho consiste no estudo de um método de identificação de parâmetros aplicado a equação de advecção-difusão no espaço bidimensional. Essa equação é resolvida utilizando o método dos volumes finitos, sendo empregada métodos de reconstrução de alta ordem em malhas não-estruturadas de triângulos para calcular os fluxos nas faces dos volumes de controle. Como ferramenta de busca dos parâmetros é empregada a técnica baseadas em gradientes, sendo os mesmos calculados utilizando processos baseados em métodos adjuntos. Um algoritmo para o reconhecimento do coeficiente de difusão foi desenvolvido e seu desempenho avaliado para uma série de variações dos valores de controle do algoritmo. 


\section{Abstract}

This work concerns a parameter identification method applied on the advection-diffusion equation in two dimensions. This equation is solved employing the finite volume and high-order reconstruction methods, on unstructured triangle meshes to solve the fluxes across the faces of control volumes. As parameter searching tool is gradient based techniques are applied. The gradients are solved using adjoint methods. An algorithm parameter identification was developed and its performance was evaluated for a series of variations of the control volumes of the algorithm. 


\section{Sumário}

Lista de Figuras . . . . . . . . . . . . . . . . . . . . . . . . . . . . . . . . . . . . . 8

Lista de Tabelas . . . . . . . . . . . . . . . . . . . . . . . . . . . . . . . . . . . . . . . . . . . 10

1 Introdução $\quad 1$

2 Método dos volumes finitos de alta ordem $\quad 11$

2.1 Método dos volumes finitos . . . . . . . . . . . . . . . . . . . . . . 11

2.2 Método de reconstrução de alta ordem . . . . . . . . . . . . . . . . . . . . . . . . . . . . 15

2.2.1 Reconstrução da solução para os volumes de controle do interior . . . . . . . . . . . . 15

2.2.2 Quadratura Gaussiana . . . . . . . . . . . . . . . . . . . . . . 21

2.2.3 Condição de fronteira de Dirichlet . . . . . . . . . . . . . . . . . . . . . . . . . . . . 24

2.2.4 Condições de fronteira de Neumann . . . . . . . . . . . . . . . . . . . . . . . . . . 27

2.3 Condição de fronteira de Robin . . . . . . . . . . . . . . . . . . . . . . . . . . . . . . . . 28

2.4 Resolução da EDP . . . . . . . . . . . . . . . . . . . . . . . . . . . . . . . . . . . . . . . . . . . . . . . . . . . 29

2.5 Validação . . . . . . . . . . . . . . . . . . . . . . . . . . . 32

2.5.1 Teste 1 - Equação de Poisson . . . . . . . . . . . . . . . . . . . . . . . . . . . 34

2.5.2 Teste 2 - canal retangular . . . . . . . . . . . . . . . . . . . . . 37

2.6 O efeito da difusão numérica f . . . . . . . . . . . . . . . . . . . . . . . . . . . . . . . 41

3 Método do operador adjunto para identificação de parâmetros 44

3.1 Definição do problema geral . . . . . . . . . . . . . . . . . . . . . . 44

3.2 Cálculo das sensibilidades . . . . . . . . . . . . . . . . . . . . . . . . . . . . 45

3.3 Obtenção da equação adjunta . . . . . . . . . . . . . . . . . . . . . . . . . . . . . . . . . . . . . 49

3.3.1 Integração da parte advectiva . . . . . . . . . . . . . . . . . . . 50 50 
3.3.2 Integração da parte difusiva . . . . . . . . . . . . . . . . . . . . . . . . 52

3.3.3 Equação adjunta . . . . . . . . . . . . . . . . . . . . . . . . . . . . . 58

3.4 Problema de teste . . . . . . . . . . . . . . . . . . . . . . . . . . . . 61

3.5 Cálculo das integrais . . . . . . . . . . . . . . . . . . . . . . . 63

4 Estudo numérico do problema de identificação de parâmetros 65

4.1 Introdução . . . . . . . . . . . . . . . . . . . . . . . . . . 65

4.2 Descrição do algoritmo . . . . . . . . . . . . . . . . . . . . . . . . . . . . . . . . 66

4.3 Problema teste 1: estimação de dois parâmetros - função linear . . . . . . . . . . . . . . . 73

4.4 Problema teste 2: estimação de três parâmetros - função quadrática . . . . . . . . . . . . . . 85

4.5 Problema teste 3: estimação de quatro parâmetros - função quadrática . . . . . . . . . . . . 91

5 Análise de sensibilidade do processo de identificação de parâmetros 98

5.1 Introdução . . . . . . . . . . . . . . . . . . . . . . . . . . . . . . . . . 98

5.2 Estudo da sensibilidade do primal . . . . . . . . . . . . . . . . . . . . . . . 100

5.3 Estudo da sensibilidade do dual . . . . . . . . . . . . . . . . . . . . . . . . . . . 101

5.4 Estudo da sensibilidade da busca unidimensional . . . . . . . . . . . . . . . . . . . . . 103

6 Conclusões $r \begin{array}{lr}105\end{array}$

$\begin{array}{lr}\text { Bibliografia } & 113\end{array}$ 


\section{Lista de Figuras}

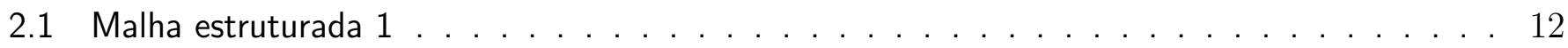

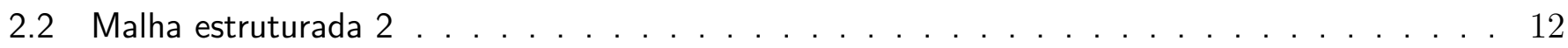

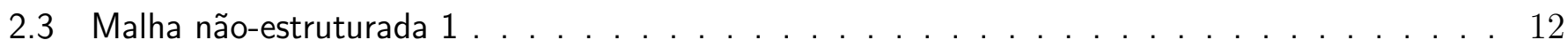

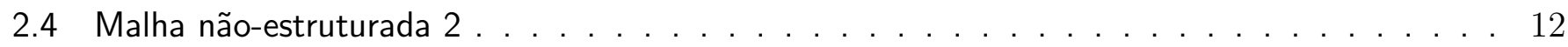

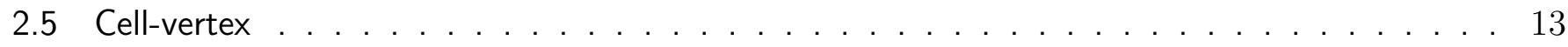

2.6 Cell-centered . . . . . . . . . . . . . . . . . . . . . . . . . . 13

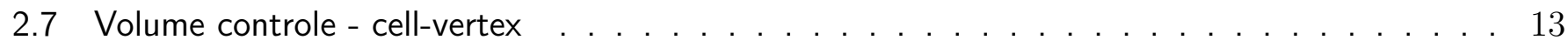

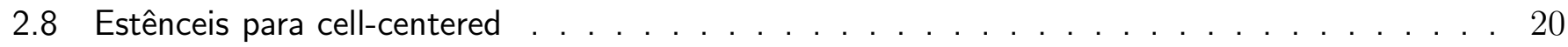

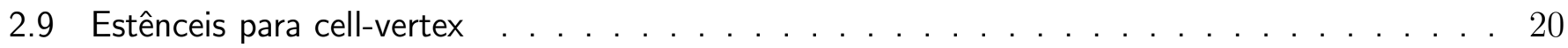

2.10 Ilustração dos pontos de Gauss na fronteira do volume de controle . . . . . . . . . . . . . . 22

2.11 pontos de Gauss - cell-vertex . . . . . . . . . . . . . . . . . . . . 25

2.12 Ilustração para o cálculo da integral de linha dos fluxos . . . . . . . . . . . . . . . 33

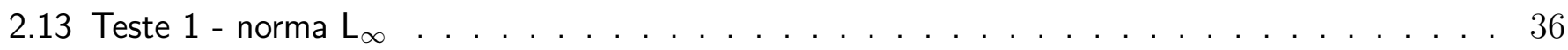

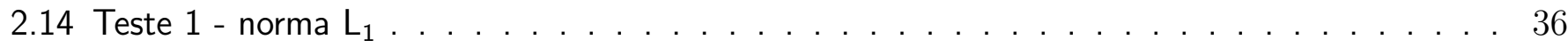

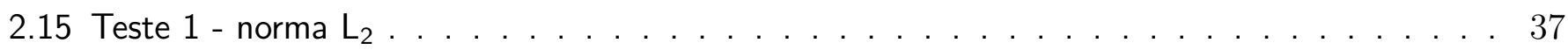

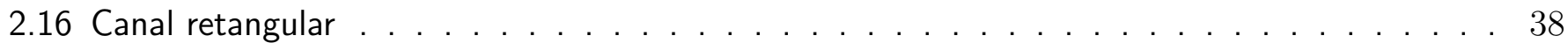

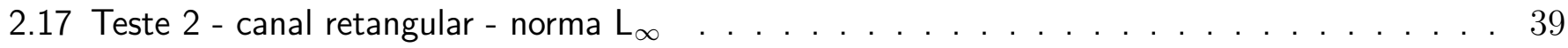

2.18 Teste 2 - canal retangular - norma $L_{1} \ldots \ldots \ldots \ldots \ldots$

2.19 Teste 2 - canal retangular - norma $\mathrm{L}_{2} \ldots \ldots \ldots \ldots \ldots \ldots$

2.20 Efeito da difusão numérica na malha $1 \ldots \ldots \ldots \ldots \ldots$

2.21 Efeito da difusão numérica na malha $3 \ldots \ldots \ldots \ldots \ldots \ldots \ldots$

4.1 Relações de dependência . . . . . . . . . . . . . . . . . . . . . . 66 
4.2 Fluxograma para o problema de identificação de parâmetros utilizando o método dos gradientes conjugados . . . . . . . . . . . . . . . . . . . . . . . 68

4.3 Solução de referência $\phi_{\mathrm{R}} \ldots \ldots \ldots$. . . . . . . . . . . . . . . . . . . . . . . . . . 75

4.4 Gráfico de $k(x, y)=0.1 x+0.1 y+0.05 \ldots \ldots \ldots \ldots \ldots \ldots$. . . . . . . . . . . 75

4.5 Gráfico de $\phi$ inicial . . . . . . . . . . . . . . . . . . . . . . . . . . 76

4.6 Termo fonte do adjunto inicial $\phi_{\mathrm{R}}-\phi \ldots \ldots \ldots \ldots \ldots \ldots$

4.7 Gráfico da solução adjunta inicial $\psi \ldots \ldots$. . . . . . . . . . . . . . . . . . . 76

4.8 Malha 1 - MGC - MRAO - ordem $3 \ldots \ldots$. . . . . . . . . . . . . . . . . . . 84

4.9 Malha 1 - MGC - MRAO - ordem $4 \ldots \ldots$. . . . . . . . . . . . . . 84

4.10 Malha 2 - MGC - MRAO - ordem $3 \ldots \ldots \ldots$. . . . . . . . . . . . . . . . . . . . 84

4.11 Malha 2 - MGC - MRAO - ordem $4 \ldots \ldots \ldots$. . . . . . . . . . . . . . . 84

4.12 Malha 3 - MGC - MRAO - ordem $3 \ldots \ldots$. . . . . . . . . . . . . . . . . . 85

4.13 Malha 3 - MGC - MRAO - ordem $4 \ldots \ldots$. . . . . . . . . . . . . . . . . . . . . . 85

4.14 Gráfico de $\phi_{\mathrm{R}} \ldots \ldots \ldots \ldots$. . . . . . . . . . . . . . . . . . . 86

4.15 Função de referência . . . . . . . . . . . . . . . . . . . . . . . . . . . . . 86

4.16 Termo fonte do dual inicial $\phi_{\mathrm{R}}-\phi \ldots \ldots \ldots$. . . . . . . . . . . . . 86

4.17 Gráfico da solução dual inicial $\psi$. . . . . . . . . . . . . . . . . . . . . . . . . . . . . 86

4.18 Gráfico de $\phi_{\mathrm{R}} \ldots \ldots \ldots \ldots$. . . . . . . . . . . . . . . . . . . . . . . . 92

4.19 Função de referência . . . . . . . . . . . . . . . . . . . . . . . . . . . . . . . . . . . . . . . . . 92

4.20 Termo fonte do dual inicial $\phi_{\mathrm{R}}-\phi \ldots \ldots \ldots$. . . . . . . . . . . . . . . 92

4.21 Gráfico da solução dual inicial $\psi$. . . . . . . . . . . . . . . . . . . . . . . . . . . . . . . . . . 92 


\section{Lista de Tabelas}

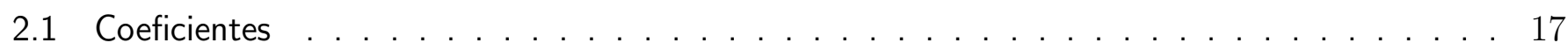

2.2 pontos de Gauss, pesos e normais para reconstrução de segunda ordem . . . . . . . . . . . 23

2.3 pontos de Gauss, pesos e normais para reconstrução de terceira ordem . . . . . . . . . . . 23

2.4 pontos de Gauss, pesos e normais para reconstrução de quarta ordem . . . . . . . . . . . . 23

2.5 Número de pontos de Gauss necessários para cada ordem de reconstrução . . . . . . . . . . . 25

2.6 Teste 1 - Parâmetros das malhas no quadrado . . . . . . . . . . . . . . . . 35

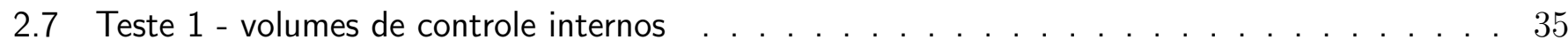

2.8 Teste 2 - Parâmetros das malhas no problema do canal retangular . . . . . . . . . . . . 38

2.9 Teste 2 - canal retangular - volumes de controle internos . . . . . . . . . . . . . . 40

2.10 Tabela com número de vértices e triângulos das malhas de triângulos . . . . . . . . . . . . 42

2.11 Tabela com número de vértices e triângulos das malhas de triângulos . . . . . . . . . . . . 42

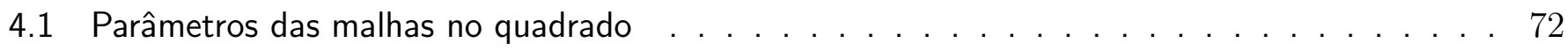

4.2 Teste 1: Descrição das tabelas . . . . . . . . . . . . . . . . . . . . . . . . . 74

4.3 Malha 1: MGC com MRAO de ordem 3 usando método adjunto . . . . . . . . . . . 77

4.4 Malha 1: MGC com MRAO de ordem 3 usando diferenças finitas . . . . . . . . . . . 77

4.5 Malha 2: MGC com MRAO de ordem 3 usando método adjunto . . . . . . . . . . 78

4.6 Malha 2: MGC com MRAO de ordem 3 usando diferenças finitas $\ldots \ldots \ldots \ldots$

4.7 Malha 3: MGC com MRAO de ordem 3 usando método adjunto . . . . . . . . . . . . . 79

4.8 Malha 3: MGC com MRAO de ordem 3 usando diferenças finitas . . . . . . . . . . 80

4.9 Malha 1: MGC com MRAO de ordem 4 usando método adjunto . . . . . . . . . . 81

4.10 Malha 1: MGC com MRAO de ordem 4 usando diferenças finitas . . . . . . . . . . . . . 81

4.11 Malha 2: MGC com MRAO de ordem 4 usando método adjunto $\ldots \ldots \ldots \ldots$. . . . . 82 
4.12 Malha 2: MGC com MRAO de ordem 4 usando diferenças finitas . . . . . . . . . . . . . . 82

4.13 Malha 3: MGC com MRAO de ordem 4 usando método adjunto . . . . . . . . . . . . . . . 83

4.14 Malha 3: MGC com MRAO de ordem 4 usando diferenças finitas . . . . . . . . . . . . . . 83

4.15 Teste 2: Descrição das tabelas . . . . . . . . . . . . . . . . . . . . . . . . . . . . . 87

4.16 Malha 1: MGC com MRAO de ordem 3 usando método adjunto - 3 parâmetros . . . . . . . . 87

4.17 Malha 1: MGC com MRAO de ordem 3 usando diferenças finitas - 3 parâmetros . . . . . . . 88

4.18 Malha 1: MGC com MRAO de ordem 4 usando método adjunto - 3 parâmetros . . . . . . . . 88

4.19 Malha 1: MGC com MRAO de ordem 4 usando diferenças finitas - 3 parâmetros . . . . . . . 88

4.20 Malha 2: MGC com MRAO de ordem 3 usando método adjunto - 3 parâmetros . . . . . . . . 89

4.21 Malha 2: MGC com MRAO de ordem 3 usando diferenças finitas - 3 parâmetros . . . . . . . 89

4.22 Malha 2: MGC com MRAO de ordem 4 usando método adjunto - 3 parâmetros . . . . . . . . 89

4.23 Malha 2: MGC com MRAO de ordem 4 usando diferenças finitas - 3 parâmetros . . . . . . . 90

4.24 Malha 3: MGC com MRAO de ordem 3 usando método adjunto - 3 parâmetros . . . . . . . . 90

4.25 Malha 3: MGC com MRAO de ordem 3 usando diferenças finitas - 3 parâmetros . . . . . . . 90

4.26 Malha 3: MGC com MRAO de ordem 4 usando método adjunto - 3 parâmetros . . . . . . . . 91

4.27 Malha 3: MGC com MRAO de ordem 4 usando diferenças finitas - 3 parâmetros . . . . . . . 91

4.28 Teste 2: Descrição das tabelas . . . . . . . . . . . . . . . . . . . . . . . . . . . 93

4.29 Malha 1: MGC com MRAO de ordem 3 usando método adjunto - 4 parâmetros . . . . . . . . 93

4.30 Malha 1: MGC com MRAO de ordem 3 usando diferenças finitas - 4 parâmetros . . . . . . . 93

4.31 Malha 1: MGC com MRAO de ordem 4 usando método adjunto - 4 parâmetros . . . . . . . . 94

4.32 Malha 1: MGC com MRAO de ordem 4 usando diferenças finitas - 4 parâmetros . . . . . . . 95

4.33 Malha 2: MGC com MRAO de ordem 3 usando método adjunto - 4 parâmetros . . . . . . . . 95

4.34 Malha 2: MGC com MRAO de ordem 3 usando diferenças finitas - 4 parâmetros . . . . . . . 95

4.35 Malha 2: MGC com MRAO de ordem 4 usando método adjunto - 4 parâmetros . . . . . . . . 96

4.36 Malha 2: MGC com MRAO de ordem 4 usando diferenças finitas - 4 parâmetros . . . . . . . 96

4.37 Malha 3: MGC com MRAO de ordem 3 usando método adjunto - 4 parâmetros . . . . . . . . 96

4.38 Malha 3: MGC com MRAO de ordem 3 usando diferenças finitas - 4 parâmetros . . . . . . . 96

4.39 Malha 3: MGC com MRAO de ordem 4 usando método adjunto - 4 parâmetros . . . . . . . . 96

4.40 Malha 3: MGC com MRAO de ordem 4 usando diferenças finitas - 4 parâmetros . . . . . . . 97 
5.1 Fatores base . . . . . . . . . . . . . . . . . . . . . . . . . . . . . 99

5.2 Descrição das tabelas para análise de sensibilidade . . . . . . . . . . . . . . . . . . . . . . 99

5.3 Parâmetros das malhas . . . . . . . . . . . . . . . . . . . . . . . . . . . . . . . . . . . . 99

5.5 Tabela com a sensibilidade dos primais . . . . . . . . . . . . . . . . . . . . . . 100

5.7 Tabela com a sensibilidade dos duais . . . . . . . . . . . . . . . . . . . . . . . . . . 102

5.9 Tabela com a sensibilidade das buscas unidimensionais . . . . . . . . . . . . . . . . . 103 


\section{Introdução}

O desenvolvimento de métodos de resolução numérica para problemas envolvendo estimação de parâmetros é uma das áreas do conhecimento humano em constante atividade. Esse campo de estudo está inserido em uma grande área conhecida como problemas inversos. Tais problemas tem por essência a necessidade de se descobrir uma causa que produziu um determinado efeito. É justamente o contrário dos chamados problemas diretos, onde se estabelece uma causa e deseja-se saber o efeito.

Problemas de estimação de parâmetros aparecem em várias áreas tecnológicas, como por exemplo:

- Em projetos de veículos espaciais [49]. O aquecimento aerodinâmico de veículos espaciais, ao reentrar na atmosfera, é tão grande que a temperatura na superfície da estrutura da nave não pode ser medida diretamente com sensores de temperatura. No estudo desse aquecimento, os sensores são colocados abaixo da referida superfície. Desse modo, o estudo da distribuição de temperatura, bem como análise das propriedades termofísicas do material de revestimento da nave, pode ser feito fazendo uma análise inversa. As medidas de temperatura aferidas pelos sensores são então utilizados para estimar a distribuição de temperatura na estrutura ao redor da aeronave.

- No controle do resfriamento de materiais formados por aço [53]. O aço é formado por ligas metálicas de ferro e carbono, e o seu o resfriamento é feito vaporizando água sobre o referido material aquecido. Para fazer o controle do resfriamento é necessário uma descrição da transferência de calor que irá ocorrer sobre a superfície do material. Em uma das equações que modelam esse problema, existe uma função chamada função de troca de calor, e estimá-la é o que irá tornar possível o controle do resfriamento.

- Na estimação da condutividade térmica e a capacidade térmica de uma amostra de peixes, utilizando o histórico térmico que vai do congelamento até o descongelamento [50]. O peixe sofre uma grande variação de temperatura em todo processo ligado a indústria de pesca, e o conhecimento dos referidos parâmetros torna possível ter um maior controle sobre a qualidade do produto. 
- Na indústria aeronáutica em projetos de otimização de formas aerodinâmicas [35]. Nesses projetos o que se busca são formas que satisfaçam determinados pré-requisitos, como por exemplo, a obtenção de uma forma de asa com baixo coeficiente de arrasto sujeito a um coeficiente de sustentação fixo.

Um problema de identificação de parâmetros, ligado a um fenômeno físico modelado por uma equação diferencial, é comumente formulado como um problema de otimização. Para exemplificar, considere que um determinando fenômeno físico seja modelado pela equação de advecção-difusão

$$
\nabla \cdot(\vec{\omega} \phi)-\nabla \cdot(\kappa \nabla \phi)=0 \quad(x, y) \in \Omega \subset \mathbb{R}^{2}
$$

onde $\vec{\omega}=(u, v) \in \mathbb{R}^{2}$ define um campo de velocidades que transporta uma propriedade $\phi$, sendo $\kappa$ o coeficiente de difusão nesse meio.

As condições de fronteira gerais podem ser dadas por

$$
\begin{cases}\phi=f, & (x, y) \in \partial \Omega_{1} \\ \nabla \phi \cdot n=g, & (x, y) \in \partial \Omega_{2}\end{cases}
$$

sendo $\partial \Omega=\partial \Omega_{1} \cup \partial \Omega_{2}$, ou seja, em uma parte da fronteira do domínio $\partial \Omega_{1}$ o valor de $\phi$ é especificado, e em outra parte $\partial \Omega_{2}$ é o fluxo da propriedade $\phi$ que é especificado.

Considere ainda que o coeficiente de difusão $\kappa$ é uma função variável no domínio da equação e que não se tem conhecimento da forma dessa função. Para estimar a função $\kappa$, considere a mesma definida por

$$
\kappa(x, y)=\sum_{\mathrm{k}=1}^{\mathrm{N}} \beta_{\mathrm{k}} C_{\mathrm{k}}(x, y)
$$

onde $C_{\mathrm{k}}(x, y)$ são funções conhecidas e $\beta_{\mathrm{k}}$ são parâmetros a serem estimados.

Note que a solução $\phi$ da equação de advecção-difusão é função das variáveis espaciais, das componentes $u$ e $v$ do vetor $\vec{\omega}$ e do coeficiente de difusão $\kappa$. Para um escoamento dado, dessas quatro variáveis, apenas $\kappa$ não é conhecida. Considerando-se uma solução de referência $\phi_{\mathrm{R}}$, a qual pode ser obtida experimentalmente ou estabelecida de acordo com os interesses, os parâmetros $\beta_{\mathrm{k}}$ podem ser estimados utilizando como ferramenta um problema de otimização, formulado por 


$$
\min : \mathcal{F}[\kappa]=\iint_{\Omega}\left(\phi-\phi_{\mathrm{R}}\right)^{2} d \Omega
$$

sujeito ao chamado problema primal

$$
\begin{gathered}
\nabla \cdot(\vec{\omega} \phi)-\nabla \cdot(\kappa \nabla \phi)=0 \quad(x, y) \in \Omega \subset \mathbb{R}^{2} \\
\qquad \begin{cases}\phi=f, & (x, y) \in \partial \Omega_{1} \\
\nabla \phi \cdot n=g, & (x, y) \in \partial \Omega_{2}\end{cases}
\end{gathered}
$$

sendo o coeficiente de difusão dado por

$$
\kappa(x, y)=\sum_{\mathrm{k}=1}^{\mathrm{N}} \beta_{\mathrm{k}} C_{\mathrm{k}}(x, y)
$$

Esse é um problema de otimização restrita, onde a restrição é dada pela equação de advecção-difusão com domínio no $\mathbb{R}^{2}$. A resolução do mesmo consiste em estimar os $\mathrm{N}$ coeficientes $\beta_{\mathrm{k}}$ presentes em (1.7) de tal modo que a função objetivo (1.4) seja minimizada e a equação (1.5) seja satisfeita. Os coeficientes estimados serão utilizados para dar uma aproximação para o coeficiente de difusão $\kappa$. Dessa forma, pode-se notar que o problema de estimação de parâmetros está inserido no problema de otimização. Pode-se também notar implícito a meta de se estabelecer uma causa para obter um efeito desejado, isto é, o que se deseja obter é quem deve ser $\kappa$, na forma da função (1.7), que produza um campo de propriedades que fique o mais próximo possível de $\phi_{\mathrm{R}}$. O efeito desejado é $\phi_{\mathrm{R}}$ e a causa $\kappa$. Isso é alcançado quando a função objetivo for minimizada.

No exemplo, o objetivo reside em estimar os coeficientes $\beta_{k}$ que determinam a função coeficiente de difusão $\kappa$, mas se poderia ter uma outra situação no qual a referida função é conhecida e o campo de velocidade não. Nessa caso, poderia se utilizar estratégia semelhante usada para a função $\kappa$ para estimar $u$ e $v$. 
Em se tratando métodos numéricos para abordar problemas de otimização, existem os que são baseados em gradientes. Nessa classe de métodos, são os gradientes que fornecem a direção de maximização ou minimização da função objetivo.

Uma das técnicas mais simples de cálculo de gradientes é a aplicação de fórmulas de diferenças finitas. Considerando-se o problema de otimização exposto anteriormente, envolvendo a equação de advecção-difusão, os gradientes, calculados por diferenças finitas de primeira ordem, por exemplo, são dados por

$$
\delta_{\beta_{\mathrm{k}}} \mathcal{F}=\frac{\mathcal{F}\left[\phi\left(\beta_{\mathrm{k}}+\delta \beta_{\mathrm{k}}\right)\right]-\mathcal{F}\left[\phi\left(\beta_{\mathrm{k}}\right)\right]}{\delta \beta_{k}} \quad \mathrm{k}=1,2, \ldots, \mathrm{N}
$$

Para calculá-los, a equação de advecção-difusão tem que resolvida para cada coeficiente $\beta_{\mathrm{k}}$ perturbado. Logo, a referida equação tem que ser resolvida, e o funcional calculado, $\mathrm{N}$ vezes para determinar todos os gradientes. Com isso tem-se idéia do custo computacional, quando o número parâmetros a serem estimados é grande, ao empregar a técnica de diferenças finitas para avaliar os gradientes.

Os chamados métodos adjuntos formam uma outra classe de métodos para obtenção de gradientes. Essa classe de métodos tem por base o emprego da solução da chamada equação adjunta como ferramenta para calcular os gradientes.

Existe uma classificação dos métodos adjuntos em dois tipos [3, 39]: método adjunto discreto e método adjunto contínuo. A diferença está no processo de obtenção da equação adjunta. Na literatura da área, encontram-se pesquisadores que tem preferência pelo adjunto discreto enquanto outros tem pelo adjunto contínuo. Giles $[14,15,19,21,23,24,25]$ é um dos que tem preferência pela formulação discreta. A partir da referência [15] tem-se uma explanação em linhas gerais sobre o método adjunto discreto, como apresentado a seguir:

\section{Método Adjunto Discreto}

Seja $I_{h}\left(\phi_{h}(\alpha), \alpha\right)$ a função objetivo discretizada e $R_{h}\left(\phi_{h}(\alpha), \alpha\right)=0$ o resíduo discretizado que surge ao aplicar um método de resolução numérica a EDP governante em um domínio $\Omega$. As variáveis $\alpha$ e $\phi$ são, respectivamente, o parâmetro a ser estimado e a solução da propriedade ligada a EDP. A idéia do método adjunto discreto é sumarizado a seguir:

Derivando $R_{h}\left(\phi_{h}(\alpha), \alpha\right)=0$ em relação a $\alpha$, temos 


$$
\frac{\partial R_{h}}{\partial \phi_{h}} \frac{d \phi_{h}}{d \alpha}+\frac{\partial R_{h}}{\partial \alpha}=0 \Rightarrow \frac{d \phi_{h}}{d \alpha}=-\left(\frac{\partial R_{h}}{\partial \phi_{h}}\right)^{-1} \frac{\partial R_{h}}{\partial \alpha}
$$

Derivando $I_{h}\left(\phi_{h}(\alpha), \alpha\right)$ em relação a $\alpha$, temos

$$
\frac{d I_{h}}{d \alpha}=\frac{\partial I_{h}}{\partial \phi_{h}} \frac{d \phi_{h}}{d \alpha}+\frac{\partial I_{h}}{\partial \alpha}
$$

Substituindo (1.9) em (1.10), segue

$$
\frac{d I_{h}}{d \alpha}=\underbrace{-\frac{\partial I_{h}}{\partial \phi_{h}}\left(\frac{\partial R_{h}}{\partial \phi_{h}}\right)^{-1}}_{\psi_{h}^{T}} \frac{\partial R_{h}}{\partial \alpha}+\frac{\partial I_{h}}{\partial \alpha} \Rightarrow \frac{d I_{h}}{d \alpha}=\psi_{h}^{T} \frac{\partial R_{h}}{\partial \alpha}+\frac{\partial I_{h}}{\partial \alpha}
$$

onde $\psi_{h}$ é a solução da equação adjunta

$$
\left(\frac{\partial R_{h}}{\partial \phi_{h}}\right)^{T} \psi_{h}+\left(\frac{\partial I_{h}}{\partial \phi_{h}}\right)^{T}=0
$$

Ao resolver a equação (1.12), o cálculo do gradiente da função $I$ em relação aos parâmetros a serem estimados ficam independentes das derivadas das funções $R$ e $I$ em relação a $\phi$. Em um processo iterativo para estimação de parâmetros, a equação (1.12) tem que ser resolvida uma única vez por iteração e seu resultado substituido em (1.11), para que junto com o cálculo dos demais fatores e parcelas, os gradientes sejam obtidos.

Jameson $[33,34,35,36,37,38]$ é um dos pesquisadores que tem preferência pela formulação contínua, cujas idéias são dadas a seguir:

\section{Método Adjunto Contínuo}

Seja $I(\phi(\alpha), \alpha)$ a função objetivo e $R(\phi(\alpha), \alpha)=0$ as EDPs que governam uma dada propriedade física em um domínio $\Omega$. A idéia do método adjunto contínuo é sumarizado a seguir:

A variação em $I(\phi(\alpha), \alpha)$ devido a uma variação em $\alpha$ é dada por 


$$
d I=\left(\frac{\partial I}{\partial \phi}\right)^{T} d \phi+\left(\frac{\partial I}{\partial \alpha}\right)^{T} d \alpha
$$

A variação em $R(\phi(\alpha), \alpha)$ devido a uma variação em $\alpha$ é dada por

$$
d R=\left[\frac{\partial R}{\partial \phi}\right] d \phi+\left[\frac{\partial R}{\partial \alpha}\right] d \alpha=0
$$

Na abordagem adjunto contínuo a função objetivo é aumentada, onde a restrição, dada pela variação $d R$, é inserida multiplicada por um $\psi^{T}$ chamado multiplicador de Lagrange. Com isso,

$$
\begin{aligned}
d I & =d I+\psi^{T} d R \\
& =\left(\frac{\partial I}{\partial \phi}\right)^{T} d \phi+\left(\frac{\partial I}{\partial \alpha}\right)^{T} d \alpha+\psi^{T}\left[\left(\frac{\partial R}{\partial \phi}\right) d \phi+\left(\frac{\partial R}{\partial \alpha}\right) d \alpha\right] \\
& =\left[\left(\frac{\partial I}{\partial \phi}\right)^{T}+\psi^{T}\left(\frac{\partial R}{\partial \phi}\right)\right] d \phi+\left[\left(\frac{\partial I}{\partial \alpha}\right)^{T}+\psi^{T}\left(\frac{\partial R}{\partial \alpha}\right)\right] d \alpha
\end{aligned}
$$

Agora, escolhendo $\psi$ como solução da equação adjunta contínua

$$
\left(\frac{\partial I}{\partial \phi}\right)^{T}+\psi^{T}\left(\frac{\partial R}{\partial \phi}\right)=0
$$

o primeiro termo é anulado, e daí segue que

$$
d I=\left[\left(\frac{\partial I}{\partial \alpha}\right)^{T}+\psi^{T}\left(\frac{\partial R}{\partial \alpha}\right)\right] d \alpha
$$

e com isso temos a variação $\frac{d I}{d \alpha}$ na função objetivo.

Novamente aqui, assim como na formulação discreta, o cálculo dos gradientes da função objetivo $I$ em relação aos parâmetros a serem estimados ficam independentes das derivadas $R$ e $I$ em relação $\phi$ e a equação adjunta precisa ser resolvida apenas uma única vez por iteração. 
Em ambas as abordagens, tanto o adjunto contínuo quanto o adjunto discreto, a solução da equação adjunta irá fornecer, de uma única vez, a variação da função objetivo em todo domínio. Conceitualmente, a essência das duas estratégias para o cálculo do gradiente função objetivo é a mesma. A diferença é que no método adjunto discreto primeiro discretiza-se a função objetivo e a equação de restrição. Em seguida obtém-se a formulação adjunta. No adjunto contínuo, primeiro obtém-se a equação adjunta e em seguida é feita a discretização desta.

No que tange as equações adjuntas, existe uma longa história no seu uso em teoria do controle [42]. Em dinâmica de fluidos, o pioneiro no uso das equações adjuntas foi Pironneau [51]. Dentre os trabalhos no campo da mecânica dos fluidos computacional, envolvendo as equações de Navier-Stokes e Euler, destacam-se os trabalhos de Jameson $[33,34,35,37,38]$. Nesses trabalhos, o autor tem como objetivo o desenvolvimento de ferramentas, baseadas nos métodos adjuntos, para aplicação em otimização de formas aerodinâmicas. Nesses problemas, os parâmetros a serem estimados são aqueles que definem a forma da aeronave de tal modo que determinadas propriedades aerodinâmicas, como por exemplo, coeficiente de arrasto ou a razão coeficiente de arrasto/sustentação, sejam minimizadas. Além desse campo de aplicação, os métodos adjuntos tem sido empregados em um outro campo conhecido como problemas inversos em tranferência de calor. Orlande [7] apresenta um problema, aplicando o método adjunto, onde o objetivo consiste em estimar simultaneamente a função que determina o coeficiente de difusão e uma outra associada ao termo fonte. Zabaras [55] apresenta um problema onde método adjunto é empregado no projeto de dispositivos de controle de processos de solidificação. Em resumo, existe uma grande quantidade de problemas inversos, encontrados na literatura da área, apresentando situações onde os métodos adjuntos podem ser aplicados no processo de estimação de parâmetros.

Além de sua aplicação em problemas de identificação de parâmetros, quando se utiliza o método adjunto, a solução da equação adjunta pode também ser empregada para aumentar a precisão de funcionais integrais, funcionando como um corretor, conforme pode ser visto nos trabalhos de Giles [22, 20, 17, 16, 18] e Darmofal $[9,10,8,11]$.

Cada uma das formulações apresentam vantagens e desvantagens. No método adjunto discreto, por exemplo, não se tem a necessidade de manipulações analíticas das equações e de suas condições de contorno. Como desvantagem tem a necessidade de manipulação de grandes sistemas esparsos. Na formulação contínua tem-se o potencial aproveitamento do mesmo algoritmo do problema primal. Como desvantagem exige-se 
um tratamento especial para as condições de contorno.

Problemas de identificação de parâmetros envolvendo equações diferenciais exigem métodos de resolução para tais equações. Nesse processo de solução entram temas importantes como o tipo de malha a ser utilizado no domínio do problema e o método de resolução numérica da equação diferencial. Em problemas envolvendo geometrias complexas, malhas não-estruturadas são mais indicadas pela facilidade de adaptação ao domínio. Nesse caso, o método para resolver a equação deve ser um método adequado para trabalhar com o referido tipo de malha. Um método adequado para resolver uma EDP em um domínio com geometria complexa, usando uma malha-não estruturada de triângulos, por exemplo, é o método dos volumes finitos (MVF), o qual trabalha com um princípio importante da física, que é a conservação da média, no volume de controle, da propriedade relativa a função incógnita na equação.

O MVF é classificado em dois tipos: MVF cell-centered e MVF cell-vertex. No primeiro, as propriedades das EDPs são armazenadas nos centróides das células. No segundo, essas propriedades são armazenadas nos vértices da malha. Como em malhas não-estruturadas de triângulos o número de vértices é menor que o número de triângulos, os esquemas de volumes finitos baseados em cell-vertex são uma boa escolha, uma vez que economizam memória.

O MVF é um método que exige a reconstrução da solução nas faces dos volumes de controle para calcular os fluxos. Para tratar isso é necessário uma técnica de reconstrução. No que tange a reconstrução, o emprego de técnicas de alta ordem são convenientes para calcular esses fluxos, pois a ordem elevada proporciona aumento na precisão da solução e também a redução nos efeitos provocados pela difusão numérica. Na literatura existem várias técnicas de reconstrução de alta ordem. ENO [31] é um exemplo de um esquema de reconstrução, essencialmente não-oscilatório, baseado sobre uma técnica especial de interpolação que, quando aplicado em dados suaves por partes, produz resultados com precisão de alta ordem em qualquer lugar onde a função é suave e além disso evita o fenômeno de Gibbs. No presente trabalho, para reconstruir os fluxos nas faces dos volumes de controle, o método escolhido foi um método de reconstrução de alta ordem baseado em mínimos quadrados desenvolvido por Gooch [30, 27, 29] e utilizado por Van Altena [2], trabalho o qual foi uma das fontes no desenvolvimento dessa tese. A técnica de reconstrução apresentada por Gooch teve por base o trabalho de Barth $[5,6]$. Em essência, como será detalhado no segundo capítulo, esse método aproxima a solução em cada volume de controle por um polinômio, e esses por sua vez são utilizados para avaliar os fluxos nas faces dos volumes de controle. 
Existem na literatura trabalhos, envolvendo os métodos adjuntos, onde as equações diferenciais de restrição e as adjuntas são resolvidas utilizando o MVF, tanto em malhas estruturadas como não-estruturadas. Jameson em [36], o qual emprega o método adjunto usando formulação contínua, resolve as equações de NavierStokes utilizando o MVF cell-centered e malhas estruturadas. Além do trabalho citado, ainda vários artigos abordando o assunto [33, 34, 35, 37, 38, 39]. Anderson [4], também usando formulação contínua, resolve as equações de Navier-Stokes utilizando o MVF cell-vertex e malhas não-estruturadas de triângulos. Nadarajah [45], utilizando formulação discreta, resolve as equações de Navier-Stokes usando o MVF cell-centered e malhas estruturadas. Elliot [13], usando formulação contínua, resolve as equações de Navier-Stokes utilizando o MVF/MEF e malhas não-estruturadas.

Contextualizando, o presente texto tem por finalidade apresentar um estudo sobre o problema de identificação de parâmetros. Esses parâmetros são os coeficientes $\beta_{k}, k=1, \ldots, N$, da função coeficiente de difusão

$$
\kappa(x, y)=C_{0}+\sum_{\mathrm{k}=1}^{\mathrm{N}} \beta_{\mathrm{k}} C_{\mathrm{k}}(x, y)
$$

presente equação de advecção-difusão

$$
\nabla \cdot(\vec{\omega} \phi)-\nabla \cdot(\kappa \nabla \phi)=0 \quad(x, y) \in \Omega
$$

onde $\Omega=[0,1] \times[0,1] \subset \mathbb{R}^{2}$ é um quadrado unitário e o campo de velocidades dado por $\vec{\omega}=(1,0)$. As condições fronteira serão especificadas no problema de teste no capítulo 4.

A função objetivo utilizada no processo é dada por

$$
\mathcal{F}[\kappa]=\iint_{\Omega}\left(\phi-\phi_{\mathrm{R}}\right)^{2} d \Omega
$$

onde $\phi_{\mathrm{R}}$ são valores de referência previamente calculados.

Os gradientes são calculados via método adjunto contínuo. Os testes são feitos empregando malhas nãoestruturadas de triângulos. A equação de restrição e sua adjunta são resolvidas usando o MVF cell-vertex. 
Para reconstruir os fluxos nas faces dos volumes de controle, é utilizado um método de reconstrução de alta ordem baseada em mínimos quadrados desenvolvido por Gooch [30].

A equação de advecção-difusão é utilizada como restrição por ser uma equação representativa de fenômenos de transporte e difusão, assim como são as equações de Navier-Stokes. O MVF foi escolhido por funcionar sobre o princípio de conservação da média, uma propriedade importante da física. A motivação pela utilização do MVF baseado em Cell-vertex foi a economia de memória uma vez que o número de vértices é menor que o número de triângulos. Embora o domínio do problema seja um quadrado, uma geometria simples, malhas não-estruturadas de triângulos foram empregadas pela facilidade de adaptação em domínios com geometrias complexas.

O capítulo 2 apresenta os fundamentos do método dos volumes finitos, o método de reconstrução de alta ordem em seus detalhes, o método de resolução de EDPs utilizando o Método de Runge-Kutta multiestágio, bem como a técnica de emprego da reconstrução utilizada dentro deste, e finaliza com testes de validação da ordem. O capítulo 3 apresenta o método do operador adjunto aplicado em problemas de identificação de parâmetros, descrevendo em detalhes o método de obtenção da equação adjunta, usando a formulação contínua, suas condições de fronteira e o cálculo do gradiente. O capítulo 4 apresenta resultados obtidos em testes computacionais na identificação de parâmetros, no caso os coeficientes que definem a função coeficiente de difusão. Esse capítulo apresenta um estudo comparativo entre os resultados obtidos com gradientes calculados via método adjunto e via diferenças finitas. O capítulo 5 apresenta um estudo da sensibilidade no problema de identificação de parâmetros. Esse estudo tem por objetivo avaliar o que ocorre quando se pertubam várias tolerâncias envolvidas nos critérios de parada em quatros instâncias, as quais são pormenorizadas no referido capítulo. Finalizando, o capítulo 6 apresenta conclusões e propostas para trabalhos futuros. 


\section{Método dos volumes finitos de alta ordem}

Esse capítulo apresenta a essência do método dos volumes finitos (MVF) e o método de reconstrução de alta ordem (MRAO) em seus detalhes bem como sua validação. Finaliza-se apresentando o método de resolução numérica da equação de advecção-difusão (EAD2D), EDP modelo desse trabalho, e nesse processo o método de integração dos fluxos advectivos e difusivos.

\subsection{Método dos volumes finitos}

Dada uma equação diferencial parcial (EDP), com suas respectivas condições iniciais e de fronteira, o MVF consiste em:

- Discretização do domínio da equação diferencial e definição dos volumes de controle: Consiste na geração de uma malha, seja estruturada, onde a localização dos nós vizinhos (das células vizinhas) a um dado nó (a uma dada célula) é determinada por variações nos índices em cada direção, conforme figuras 2.1 e 2.2 ,

ou não-estruturada, onde a localização dos nós vizinhos (das células vizinhas) a um dado nó (a uma dada célula) é determinada por uma estrutura de dados, conforme figuras 2.3 e 2.4 .

Com o domínio discretizado em um número finito de nós, dentro do mesmo são definidos um número finito de volumes de controle. Os esquemas baseados em MVF são classificados em dois tipos: cellvertex (figura 2.5) e cell-centered (figura 2.6).

No primeiro tipo as informações da função incógnita são armazenadas nos próprios nós da malha. No segundo tipo, as informações são armazenadas nos centróides das células da malha. O domínio espacial abordado nesse trabalho é bidimensional e as figuras apresentadas ilustram malhas estruturadas e não-estruturadas em um espaço bidimensional, mas o MVF pode ser também aplicado para 


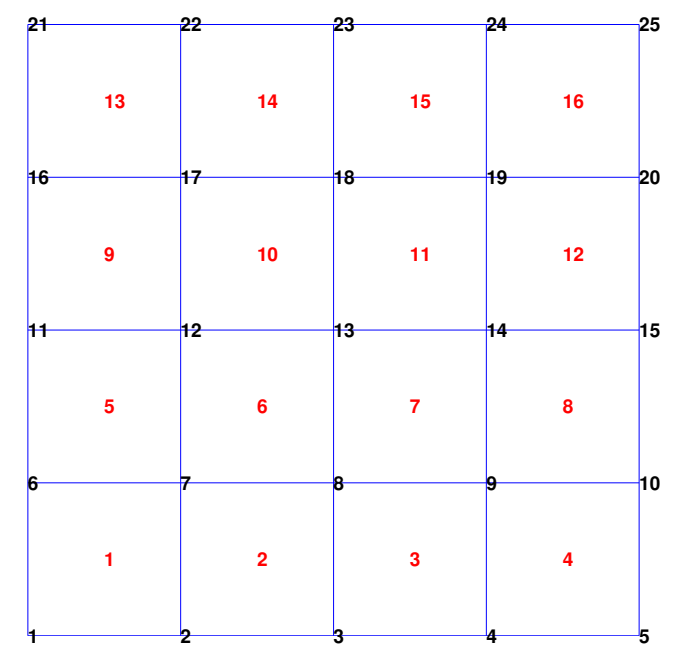

Figura 2.1: Malha estruturada 1

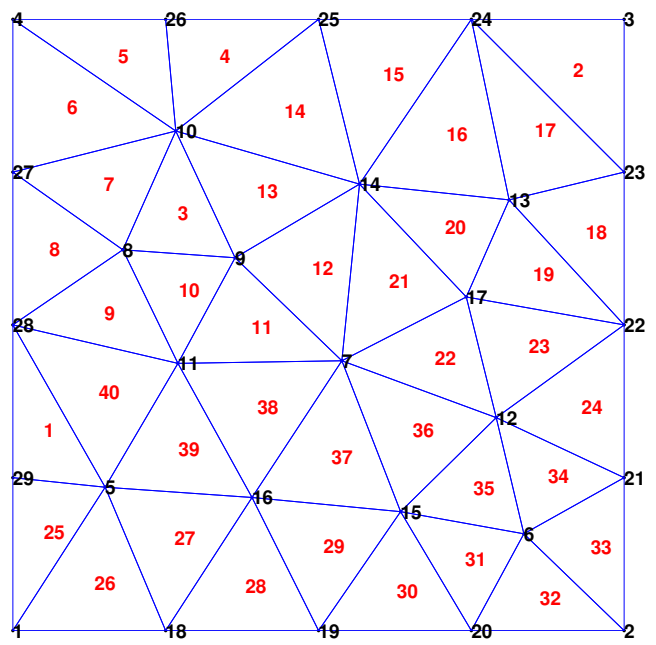

Figura 2.3: Malha não-estruturada 1

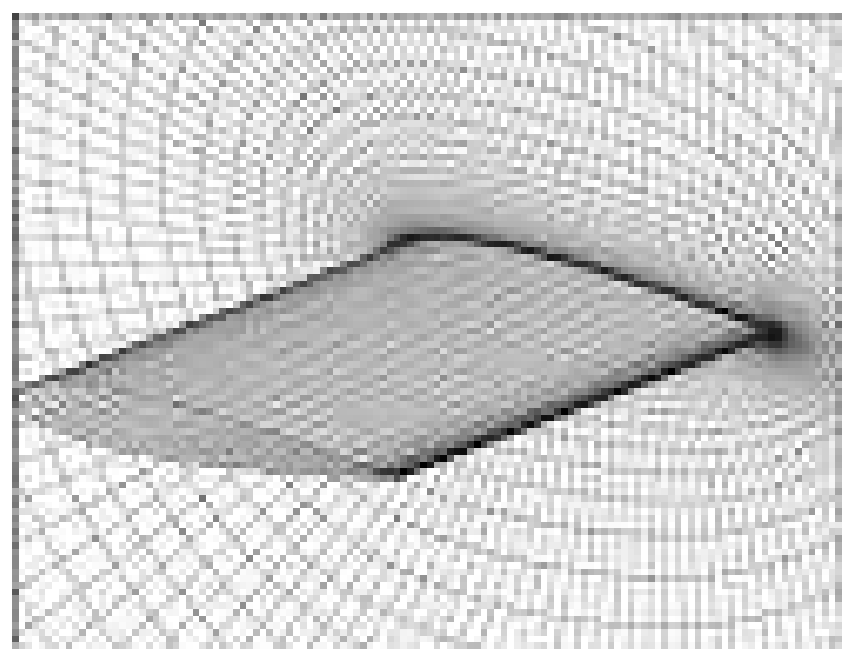

Figura 2.2: Malha estruturada 2

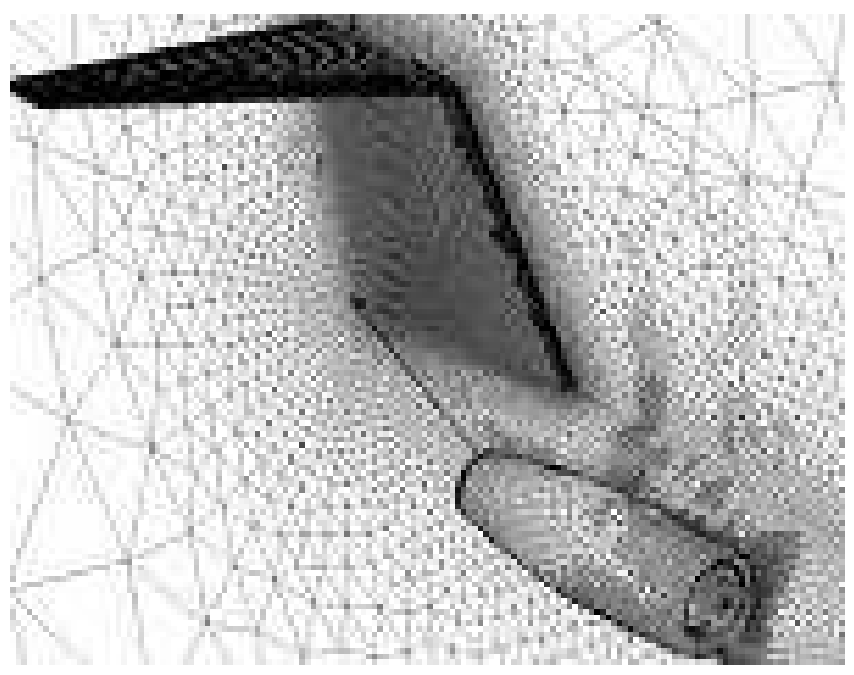

Figura 2.4: Malha não-estruturada 2

domínios tridimensionais e nesse caso os volumes de controle poderiam ser tetraedros, cubos, prismas ou pirâmides.

- Aproximações das integrais: O método dos volumes finitos é baseado em formulação integral das equações diferenciais, sendo que o que geralmente ocorre são integrais de linha, área, superfície e volume. Essas integrais devem ser calculadas em cada volume de controle por alguma técnica de integração numérica. Após a aplicação de alguma dessas técnicas a equação integral se transforma em uma equação algébrica, uma equação discretizada. As regras de quadratura gaussianas são as técnicas 


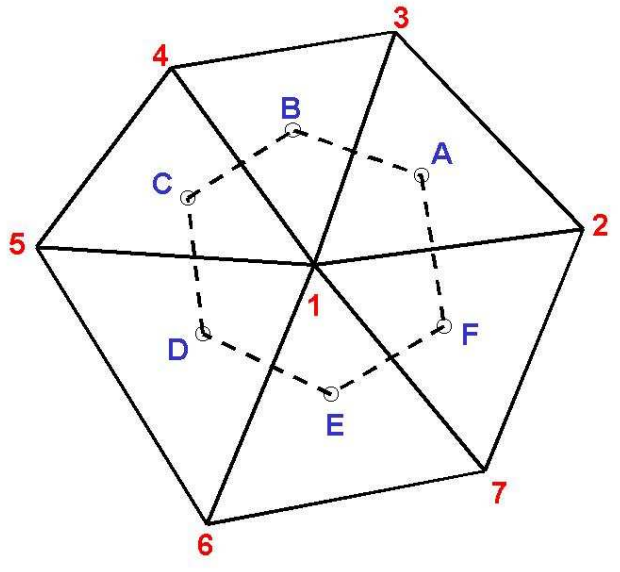

Figura 2.5: Cell-vertex

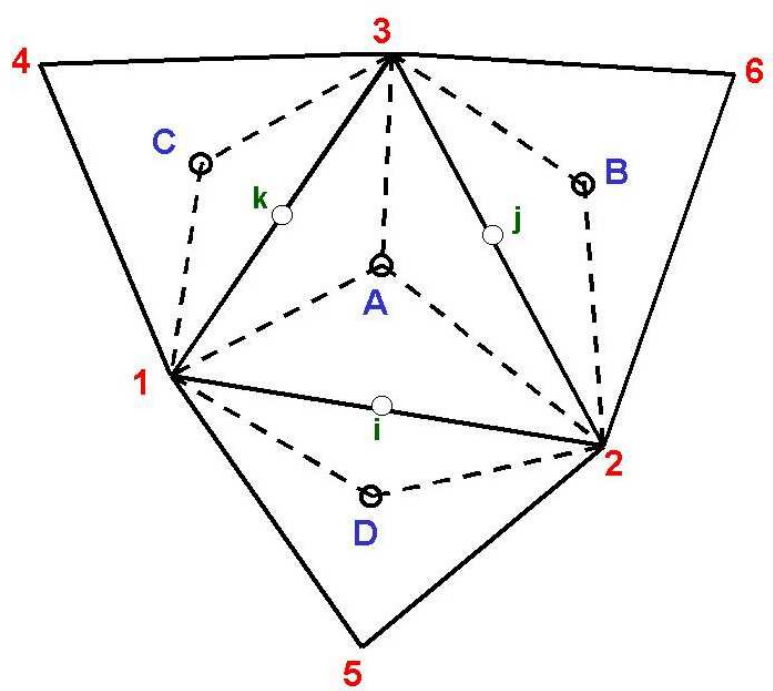

Figura 2.6: Cell-centered

de integração numérica comumente nesse processo por fornecerem boas aproximações com baixo custo computacional. Para exemplificar, considere a figura 2.7.

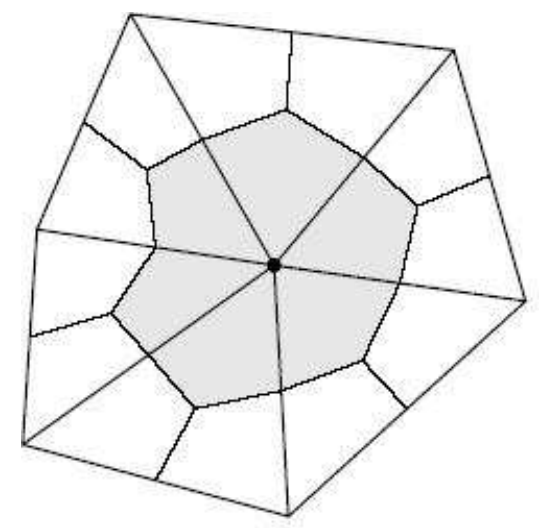

Figura 2.7: Volume controle - cell-vertex

Essa figura apresenta um volume de controle baseado em cell-vertex. Supondo que seja um volume de controle de um domínio onde a equação de Laplace, com função incógnita $\phi$, precisa ser resolvida usando o MVF, tem-se

$$
\nabla^{2} \phi=0
$$


Integrando no volume de controle, segue

$$
\iint_{\Omega} \nabla^{2} \phi d \Omega=0
$$

Usando o teorema da divergência de Gauss,

$$
\oint_{\partial \Omega} \nabla \phi \cdot n d s=0
$$

Com isso tem-se uma formulação integral da equação.

O MVF exige a integração das equações nas faces dos volume de controle. Considerando os dois tipos de esquemas dos métodos de volumes finitos, tanto cell-vertex quanto cell-centered, as integrais dos fluxos precisam ser avaliadas nas faces dos volumes controle antes de aplicar alguma técnica de integração. As aproximações nas faces podem ser obtidas de diversas maneiras, sendo a interpolação baseada em esquemas upwind, para os termos convectivos, e média dos gradientes dos valores nodais para os termos difusivos, uma das técnicas utilizadas. A qualidade das aproximações obtidas pelo MVF dependem basicamente de dois fatores: método de reconstrução da solução nas faces a partir dos valores nodais da função incógnita e do método de integração numérica, o qual utiliza os valores da solução reconstruída nas faces do volume de controle. Em essência, quanto maior a ordem de precisão da reconstrução da solução nas faces e maior for ordem de precisão do método de integração numérica, mais precisa vai ser a solução da equação diferencial nos nós do dominio discretizado. Em problemas reais, como por exemplo, em projetos aerodinâmicos, o domínio discretizado envolve milhões de nós. Com isso tem-se idéia do esforço computacional empregado em problemas dessa natureza. Assim sendo, seja em problemas transientes ou estacionários, encontrar um ponto de equilíbrio que forneça soluções precisas com custo computacional razoável é um desafio constante.

Uma das vantagens do MVF reside na garantia da lei de conservação das propriedades físicas envolvidas. Isso ocorre por que, considerando dois volumes de controle com uma face em comum, o fluxo da propriedade que sai de um volume por essa face é o mesmo que entra no outro. Com isso a média da propriedade é 
preservada. O método dos volumes finitos é adequado a qualquer forma de célula computacional, o que o faz adequado para malhas não-estruturadas. A união dessas duas vantagens faz com que o MVF seja um método conveniente para resolver problemas em mecânica de fluidos envolvendo geometrias complexas e problemas envolvendo transferência de calor e massa. Devido a tudo isso, fato que pode ser observado, o MVF aparece em muitos pacotes computacionais para resolver problemas em mecânica de fluidos computacional.

\subsection{Método de reconstrução de alta ordem}

Considere o valor médio de uma função com valores nos pontos de fronteira e nos nós de uma malha não-estruturada de triângulos dentro de um domínio de interesse. Em essência, o método de reconstrução de alta ordem desenvolvido por Gooch [30], o qual é abordado nesse trabalho, consiste em aproximar a função, em cada volume de controle, por um polinômio baseado na série de Taylor da solução. Para obter esses polinômios, os seus coeficientes, que são derivadas parciais da função, precisam ser determinados. Esses coeficientes são determinados de modo que o valor médio da solução em cada volume de controle seja preservado. Assim sendo, a ordem de precisão da reconstrução vai depender do número de termos da série de Taylor utilizada.

As próximas subseções apresentam detalhamentos no que tange ao método de reconstrução de alta ordem elaborado por Gooch [30]. Começa-se com a reconstrução nos volumes de controle do interior e depois o tratamento da reconstrução na fronteira para cada um dos três tipos dos mesmos (Dirichlet, Neumann e Robin).

\subsubsection{Reconstrução da solução para os volumes de controle do interior}

A idéia do método de reconstrução de alta ordem em questão, independente do MVF ser baseado em cell-vertex ou cell-centered, reside em descrever a função $\phi_{i}$ dentro do volume de controle por uma expansão em Série de Taylor da forma

$$
\begin{aligned}
\phi_{i}^{\mathrm{R}}(x, y)=\left.\phi\right|_{i}+\left.\frac{\partial \phi}{\partial x}\right|_{i}\left(x-x_{i}\right)+\left.\frac{\partial \phi}{\partial y}\right|_{i}\left(y-y_{i}\right)+\left.\frac{1}{2} \frac{\partial^{2} \phi}{\partial x^{2}}\right|_{i}\left(x-x_{i}\right)^{2}+ \\
\left.\quad \frac{\partial^{2} \phi}{\partial x \partial y}\right|_{i}\left(x-x_{i}\right)\left(y-y_{i}\right)+\left.\frac{1}{2} \frac{\partial^{2} \phi}{\partial y^{2}}\right|_{i}\left(y-y_{i}\right)^{2}+\left.\frac{1}{6} \frac{\partial^{3} \phi}{\partial x^{3}}\right|_{i}\left(x-x_{i}\right)^{3}+\ldots
\end{aligned}
$$


onde $\phi_{i}$ é o valor da solução reconstruída e

$$
\left.\frac{\partial^{k+l} \phi}{\partial x^{k} \partial y^{l}}\right|_{i}
$$

são suas derivadas parciais com relação ao ponto $\left(x_{i}, y_{i}\right)$ do volume de controle $i$.

Assim sendo, reconstruir a solução usando essa idéia significa aproximar a solução exata por um polinômio em duas variáveis. Para determinar esses polinômios é necessário obter os coeficientes, que são as aproximações das derivadas parciais presentes na expansão. Esses coeficientes são escolhidos de modo a preservar o valor médio dentro do volume de controle, uma vez que esse é um dos princípios do MVF, e minimizar o erro na representação de soluções suaves, de funções de classe $\mathcal{C}$. Tendo obtido os coeficientes usando esses princípios, a solução reconstruída, ou seja, o polinômio aproximador, pode ser utilizada para calcular aproximações da solução exata, bem como de suas derivadas, em qualquer ponto dentro do volume de controle.

Para que o valor médio $\bar{\phi}_{i}$ da solução $\phi_{i}$ dentro do volume de controle $i$ seja conservado, é necessário que

$$
\frac{1}{\mathrm{~A}_{i}} \iint_{\mathrm{A}_{i}} \phi_{i}^{\mathrm{R}} d \mathrm{~A}=\bar{\phi}_{i}
$$

Substituindo a expansão em Série de Taylor (2.1) em (2.2), tem-se

$$
\frac{1}{\mathrm{~A}_{i}} \iint_{\mathrm{A}_{i}}\left[\left.\phi\right|_{i}+\left.\frac{\partial \phi}{\partial x}\right|_{i}\left(x-x_{i}\right)+\left.\frac{\partial \phi}{\partial y}\right|_{i}\left(y-y_{i}\right)+\left.\frac{1}{2} \frac{\partial^{2} \phi}{\partial x^{2}}\right|_{i}\left(x-x_{i}\right)^{2}+\ldots\right] d \mathrm{~A}=\bar{\phi}_{i}
$$

que pode ser escrito na forma

$$
\left.\phi\right|_{i}+\left.\frac{\partial \phi}{\partial x}\right|_{i} \bar{x}+\left.\frac{\partial \phi}{\partial y}\right|_{i} \bar{y}+\left.\frac{1}{2} \frac{\partial^{2} \phi}{\partial x^{2}}\right|_{i} \overline{x^{2}}+\left.\frac{\partial^{2} \phi}{\partial x \partial y}\right|_{i} \overline{x y}+\left.\frac{1}{2} \frac{\partial^{2} \phi}{\partial y^{2}}\right|_{i} \overline{y^{2}}+\cdots=\bar{\phi}_{i}
$$

onde 


$$
\overline{x^{n} y^{m}}{ }_{i}=\frac{1}{\mathrm{~A}_{i}} \iint_{\mathrm{A}_{i}}\left(x-x_{i}\right)^{n}\left(y-y_{i}\right)^{m} d \mathrm{~A}
$$

O termo (2.4) que aparece em (2.3) é chamado momento e o cálculo de seu valor é obtido por regras de quadratura, as quais serão apresentadas posteriormente. A equação (2.3) é muito importante na abordagem desse método e será utilizada mais adiante.

A precisão da reconstrução da solução depende do número de termos do polinômio (2.1). No que tange a precisão para funções suaves, uma reconstrução é dita ser $k$-exata, ou tem precisão de ordem $k+1$, quando tem-se válido que $P(\vec{x}) \in\left\{x^{m} y^{n}: m+n \leq k\right\}$. Com isso, temos a seguinte equivalência

$$
\phi_{i}^{\mathrm{R}}(\vec{x}) \equiv P(\vec{x})
$$

Para obter uma reconstrução com uma determinada ordem, um certo número de coeficientes, que são as derivadas parciais da expansão (2.1), precisam ser obtidos. Considerando reconstruções de $2^{\underline{a}}$, $3^{\underline{a}}$ e $4^{\mathrm{a}}$ ordens, temos, 3, 6 e 9, respectivamente, coeficientes para serem calculados. A tabela abaixo apresenta esses coeficientes.

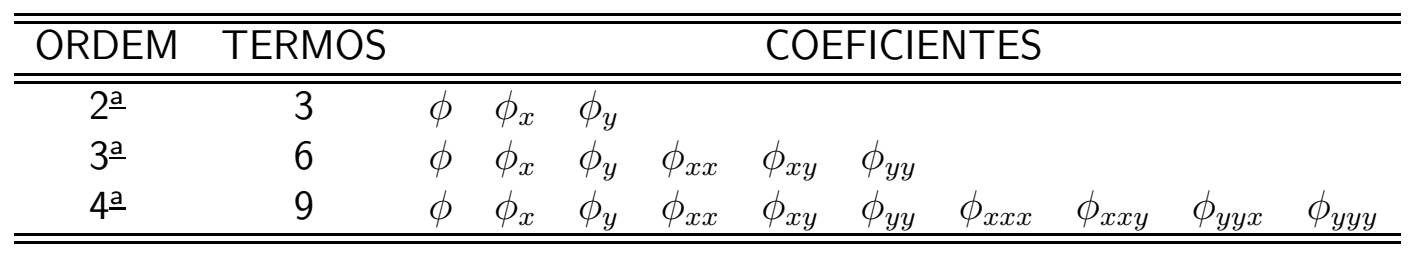

Tabela 2.1: Coeficientes

Os coeficientes são obtidos resolvendo-se um sistema sobredeterminado. No que tange ao método em questão, esse sistema é montado considerando dois princípios:

- A conservação da média $\bar{\phi}_{i}$ tem que ser satisfeita dentro do volume de controle. Logo, para isso acontecer, a equação (2.3) tem que ser uma das equações. Nesse caso é sempre a primeira equação. O motivo disso será comentado mais adiante.

- As outras equações a serem agregadas são obtidas considerando que o valor médio do polinômio de reconstrução $\phi_{i}^{\mathrm{R}}$ do volume de controle $i$ nos volumes de controle $j$ vizinhos sejam preservados. 
Assim sendo, para que o segundo item seja garantido, é necessário então que

$$
\frac{1}{\mathrm{~A}_{j}} \iint_{\mathrm{A}_{j}} \phi_{i}^{\mathrm{R}} d \mathrm{~A}=\bar{\phi}_{j}
$$

Novamente, substituindo (2.1) em (2.5), temos

$$
\begin{gathered}
\frac{1}{\mathrm{~A}_{j}} \iint_{\mathrm{A}_{j}}\left[\left.\phi\right|_{i}+\left.\frac{\partial \phi}{\partial x}\right|_{i}\left(x-x_{i}\right)+\left.\frac{\partial \phi}{\partial y}\right|_{i}\left(y-y_{i}\right)+\left.\frac{1}{2} \frac{\partial^{2} \phi}{\partial x^{2}}\right|_{i}\left(x-x_{i}\right)^{2}+\ldots\right] d \mathrm{~A}=\bar{\phi}_{j} \\
\left.\phi\right|_{i}+\left.\left[\frac{1}{\mathrm{~A}_{j}} \iint_{\mathrm{A}_{j}}\left(x-x_{i}\right) d \mathrm{~A}\right] \frac{\partial \phi}{\partial x}\right|_{i}+\left.\left[\frac{1}{\mathrm{~A}_{j}} \iint_{\mathrm{A}_{j}}\left(y-y_{i}\right) d \mathrm{~A}\right] \frac{\partial \phi}{\partial y}\right|_{i}+\left.\left[\frac{1}{2 \mathrm{~A}_{j}} \iint_{\mathrm{A}_{j}}\left(x-x_{i}\right)^{2} d \mathrm{~A}\right] \frac{\partial^{2} \phi}{\partial x^{2}}\right|_{i}+ \\
{\left.\left[\frac{1}{\mathrm{~A}_{j}} \iint_{\mathrm{A}_{j}}\left(x-x_{i}\right)\left(y-y_{i}\right) d \mathrm{~A}\right] \frac{\partial \phi}{\partial x \partial y}\right|_{i}+\left.\left[\frac{1}{2 \mathrm{~A}_{j}} \iint_{\mathrm{A}_{j}}\left(y-y_{i}\right)^{2} d \mathrm{~A}\right] \frac{\partial^{2} \phi}{\partial y^{2}}\right|_{i}+\cdots=\bar{\phi}_{j}}
\end{gathered}
$$

Substituindo $x-x_{i}$ e $y-y_{i}$, respectivamente, por $\left(x-x_{j}\right)+\left(x_{j}-x_{i}\right)$ e $\left(y-y_{j}\right)+\left(y_{j}-y_{i}\right)$, e depois expandindo em série de Taylor e integrando, segue

$$
\begin{aligned}
& \left.\phi\right|_{i}+\left.\left[\bar{x}_{j}+\left(x_{j}-x_{i}\right)\right] \frac{\partial \phi}{\partial x}\right|_{i}+\left.\left[\bar{y}_{j}+\left(y_{j}-y_{i}\right)\right] \frac{\partial \phi}{\partial y}\right|_{i}+\left.\left[\frac{\overline{x^{2}}{ }_{j}+2 \bar{x}_{j}\left(x_{j}-x_{i}\right)-\left(x_{j}-x_{i}\right)^{2}}{2}\right] \frac{\partial^{2} \phi}{\partial x^{2}}\right|_{i}+ \\
& {\left.\left[\overline{x y}_{j}+\bar{x}_{j}\left(y_{j}-y_{i}\right)+\left(x_{j}-x_{i}\right) \bar{y}_{j}+\left(x_{j}-x_{i}\right)\left(y_{j}-y_{i}\right)\right] \frac{\partial \phi}{\partial x \partial y}\right|_{i}+} \\
& {\left.\left[\frac{\bar{y}_{j}^{2}+2 \bar{y}_{j}\left(y_{j}-y_{i}\right)-\left(y_{j}-y_{i}\right)^{2}}{2}\right] \frac{\partial^{2} \phi}{\partial x^{2}}\right|_{i}+\cdots=\bar{\phi}_{j}}
\end{aligned}
$$

Os termos geométricos na equação tem a seguinte forma geral

$$
{\widehat{x^{n} y^{m}}}_{i j} \equiv \frac{1}{\mathrm{~A}_{j}} \iint_{\mathrm{A}_{j}}\left[\left(x-x_{j}\right)+\left(x_{j}-x_{i}\right)\right]^{n}\left[\left(y-y_{j}\right)+\left(y_{j}-y_{i}\right)\right]^{n} d \mathrm{~A}
$$




$$
{\widehat{x^{n} y^{m}}}_{i j}=\sum_{l=0}^{m} \sum_{k=0}^{n}\left(\begin{array}{l}
m \\
l
\end{array}\right)\left(\begin{array}{l}
n \\
k
\end{array}\right)\left(x_{j}-x_{i}\right)^{k}\left(y j-y_{i}\right)^{l} \overline{x^{n-k} y^{m-l}} j
$$

Utilizando esse resultado, segue

$$
\left.\phi\right|_{i}+\left.\widehat{x}_{i j} \frac{\partial \phi}{\partial x}\right|_{i}+\left.\widehat{y}_{i j} \frac{\partial \phi}{\partial y}\right|_{i}+\left.\widehat{x}^{2} \frac{1}{2} \frac{\partial^{2} \phi}{\partial x^{2}}\right|_{i}+\left.\widehat{x y}_{i j} \frac{\partial \phi}{\partial x \partial y}\right|_{i}+\left.\widehat{y}^{2} i \frac{1}{2} \frac{\partial^{2} \phi}{\partial y^{2}}\right|_{i}+\cdots=\bar{\phi}_{j}
$$

A equação (2.7) representa o valor médio do polinômio de reconstrução $\phi_{i}^{\mathrm{R}}(x, y)$ no volume de controle $j$ vizinho. Assim sendo, usando a equação restrição (2.3) e as equações (2.7) pode-se montar um sistema linear sobredeterminado com a seguinte forma

$$
\left[\begin{array}{ccccccc}
1 & \bar{x}_{i} & \bar{y}_{i} & \bar{x}^{2}{ }_{i} & \overline{x y}_{i} & \bar{y}^{2}{ }_{i} & \cdots \\
\hline w_{i 1} & w_{i 1} \widehat{x}_{i 1} & w_{i 1} \widehat{y}_{i 1} & w_{i 1} \widehat{x}^{2}{ }_{i 1} & w_{i 1} \widehat{x y}_{i 1} & w_{i 1} \widehat{y}^{2}{ }_{i 1} & \cdots \\
w_{i 2} & w_{i 2} \widehat{x}_{i 2} & w_{i 2} \widehat{y}_{i 2} & w_{i 2} \widehat{x}^{2}{ }_{i 2} & w_{i 2} \widehat{x y}_{i 2} & w_{i 2} \widehat{y}^{2}{ }_{i 2} & \cdots \\
w_{i 3} & w_{i 3} \widehat{x}_{i 3} & w_{i 3} \widehat{y}_{i 3} & w_{i 3} \widehat{x}^{2}{ }_{i 3} & w_{i 3} \widehat{x y}_{i 3} & w_{i 3} \widehat{y}^{2}{ }_{i 3} & \cdots \\
\vdots & \vdots & \vdots & \vdots & \vdots & \vdots & \ddots \\
w_{i N} & w_{i N} \widehat{x}_{i N} & w_{i N} \widehat{y}_{i N} & w_{i N} \widehat{x}^{2}{ }_{i N} & w_{i N} \widehat{x y}_{i N} & w_{i N}{\widehat{y^{2}}}_{i N} & \cdots
\end{array}\right]\left[\begin{array}{c}
\phi \\
\phi_{x} \\
\phi_{y} \\
\frac{1}{2} \phi_{x x} \\
\phi_{x y} \\
\frac{1}{2} \phi_{y y} \\
\vdots
\end{array}\right]_{i}=\left[\begin{array}{c}
\bar{\phi}_{i} \\
\hline w_{i 1} \bar{\phi}_{1} \\
w_{i 2} \bar{\phi}_{2} \\
w_{i 3} \bar{\phi}_{3} \\
\vdots \\
w_{i N} \bar{\phi}_{N}
\end{array}\right]_{i}
$$

onde $N$ é o número de volumes de controle vizinhos pertencentes ao estêncil. As figuras 2.8 e 2.9 mostram os estêncieis para reconstrução de ordens 2, 3 e 4 .

A primeira equação tem como finalidade assegurar a conservação da média da propriedade associada a $\phi$ dentro do volume de controle. Os pesos $w_{i j}$ usados no sistema vem de Barth [6] e são dados por

$$
w_{i j}=\frac{1}{\left|\overrightarrow{x_{j}}-\overrightarrow{x_{i}}\right|^{2}}
$$

onde $w_{i j}$ é a distância do vértice $i$ ao vértice $j$ e $\vec{x}_{i}$ é o vetor que define as coordenadas do vértice $i$. tendo por finalidade ponderar pelo inverso da distância as informações na reconstrução da solução. Isso quer dizer que quanto mais longe o volume $j$ estiver do volume de controle $i$, menos influência ele terá na obtenção 


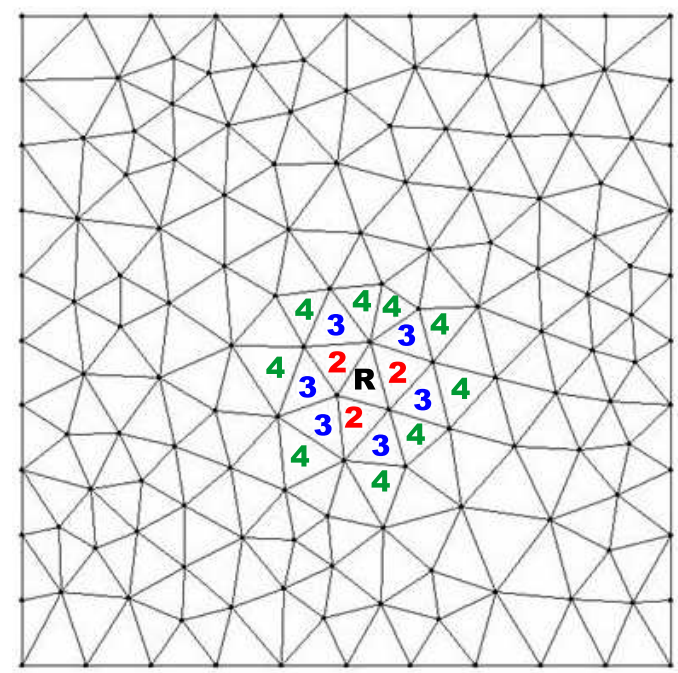

Figura 2.8: Estênceis para cell-centered

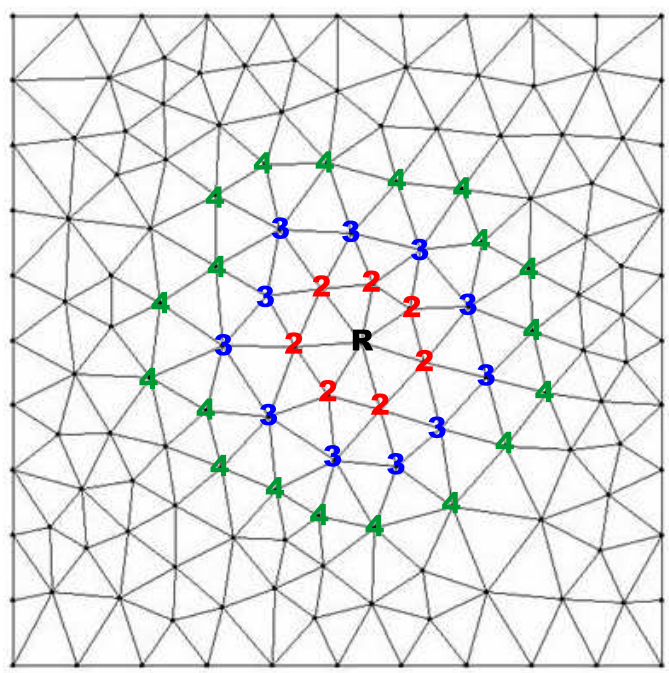

Figura 2.9: Estênceis para cell-vertex

dos coeficientes na reconstrução da solução no volume de controle $i$. Dessa forma o método leva em mais consideração os valores das médias $\bar{\phi}$ nos volumes de controle mais próximos.

A reconstrução é um polinômio baseado em série Taylor, e em se tratando desta série sabe-se que quanto maior for a ordem da expansão maior será a precisão dentro do volume de controle. Assim sendo, quanto maior for a ordem da reconstrução maior será a quantidade de nós vizinhos necessários para obter as derivadas.

Os vizinhos utilizados são os que estão mais próximos a um dado volume de controle, onde a solução será reconstruída, e o número destes que são necessários para realizar a reconstrução é igual ao número de derivadas que precisam ser obtidas na série.

Na prática necessita-se de três vizinhos para reconstrução de segunda ordem, nove para terceira ordem e quatorze para quarta ordem. As figuras 2.8 e 2.9 ilustram os vértices necessários para cada uma das ordens e para cada um dos tipos de MVF. Essas são as quantidades mínimas de nós vizinhos para cada uma das referidas ordens, mas esse número pode ser excedido. Por exemplo, para reconstrução de terceira ordem o número mínimo de nós vizinhos necessários é nove, mas se tomarmos os quinze vizinhos mais próximos o que vai ocorrer é o aumento do número de equações no sistema linear, uma quantidade maior do que o necessário. Dessa forma o sistema fica sobredeterminado e exige portanto um método adequado para esse tipo de sistema. Para contornar esse problema, de reconstruir a solução em cada volume de controle, a saída consiste em se obter uma solução de tal modo que o lado esquerdo de (2.8) forneça um resultado o mais próximo possível ao correspondente resultado do lado direito. Essa idéia de proximidade caracteriza o conceito de se obter uma solução no sentido dos mínimos quadrados. 
Existe uma vantagem ao exceder o número de nós utilizados. Essa quantidade a mais de equações no sistema aumenta o grau de liberdade para o método dos mínimos quadrados e aumenta as possibilidades de se obter uma boa reconstrução. Isso ocorre devido ao aumento do número de informações agregadas na reconstrução da solução para um dado volume de controle. Paga-se um preço em termos de esforço computacional na resolução dos sistemas, mas ganha-se em qualidade.

Considerando a reconstrução nos volumes de controle do interior, Gooch [30] apresenta uma estratégia que tem por finalidade melhorar a qualidade da reconstrução. Essa estratégia consiste em aplicar eliminação gaussiana na primeira coluna do sistema. Feito isso, parte para resolução do sistema por alguma técnica de mínimos quadrados. Gooch adota transformações de Householder para transformar o lado esquerdo do sistema linear em uma matriz triangular superior. Trabalhar com transformações de Householder significa trabalhar com transformações ortogonais e tais operações são inerentemente estáveis [26] e é esse o motivo pelo qual Gooch empregou tal método para tratar a resolução do sistema linear. A fase final, para obter a solução, consiste em aplicar o método da substituição regressiva no sistema triangular.

\subsubsection{Quadratura Gaussiana}

No método dos volumes finitos a aplicação de técnicas de integração numérica é uma constante. Nesse contexto, os métodos de integração numérica baseados em quadratura gaussiana são os métodos utilizados, por ter a vantagem de fornecerem aproximações precisas com um baixo custo computacional.

No método de reconstrução de alta ordem pode-se notar que para calcular os momentos (2.4), e em conseqüência destes, os termos geométricos (2.6), é necessário calcular integrais de polinômios em cada volume de controle. Além disso, o método dos volumes finitos tem-se a necessidade de calcular, em cada volume de controle, a integral dos fluxos advectivos e difusivos. Dependendo do problema tem-se ainda o cálculo das integrais dos termos fonte, também para cada volume de controle. Com isso, percebe-se a importância das técnicas de integração numérica.

Os tipos de integrais que ocorrem no presente trabalho são os seguintes:

- Integrais de linha:

$$
\oint_{\partial \Omega} \phi(x, y) n_{x} d s \quad \oint_{\partial \Omega} \phi(x, y) \nabla \psi(x, y) \cdot n d s
$$




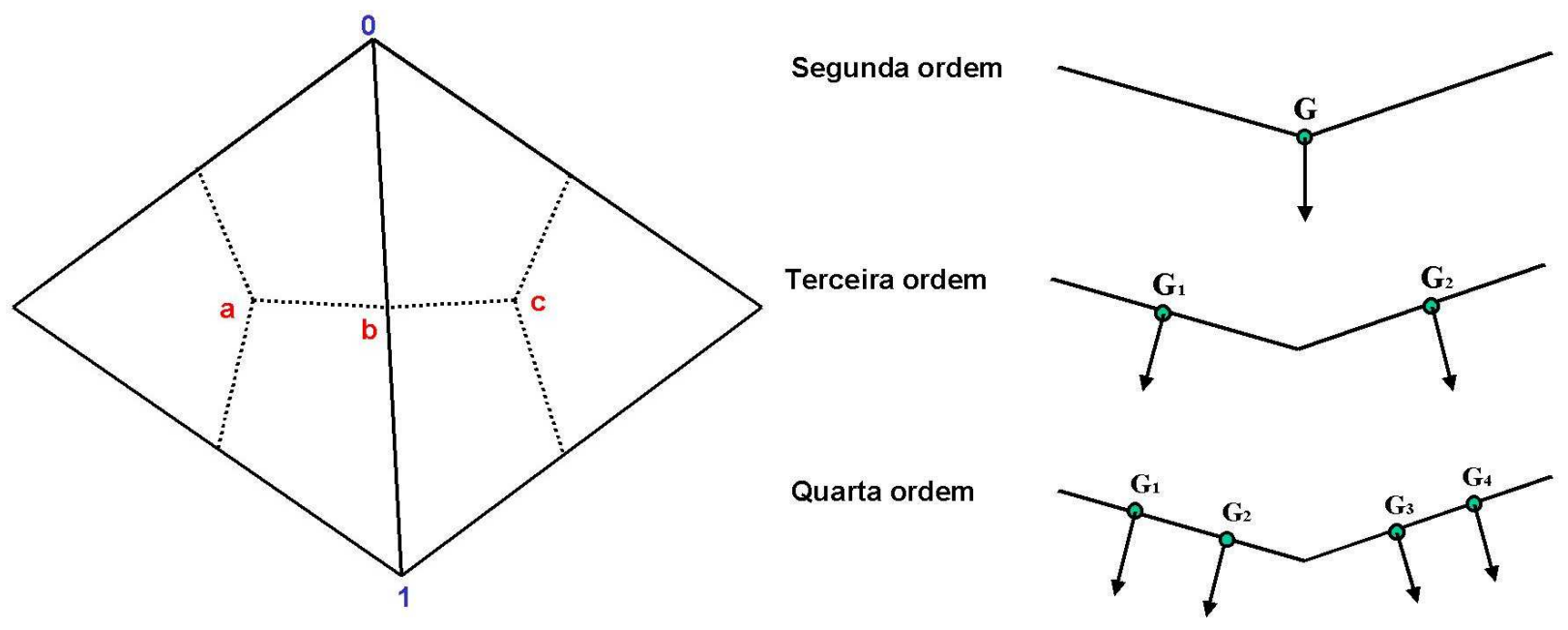

Figura 2.10: Ilustração dos pontos de Gauss na fronteira do volume de controle

- Integrais de área:

$$
\iint_{\Omega} \phi(x, y) d \Omega
$$

As funções $\phi$ e $\psi$ podem ser polinômios ou outros tipos de funções.

Para avaliar as integrais de linha, utiliza-se o método de quadratura gaussiana empregado em Van Altena [2]. Como a reconstrução da função incógnita na EDP é um polinômio, a integração exata deste vai depender do grau do mesmo. Os pontos $\mathrm{G}_{1}, \mathrm{G}_{2}, \mathrm{G}_{3}$ e $\mathrm{G}_{4}$ na figura 2.10 são os pontos de Gauss, nas faces do volume de controle, utilizados para calcular integrais de linha via quadratura gaussiana. Como pode ser observado na referida figura, por exemplo, para reconstrução de quarta ordem são necessários quatro pontos de Gauss. Tem-se ainda que a cada ponto de gauss está associado um peso e uma normal. Todas essas informações são detalhadas mais adiante.

Considerando uma face de um volume de controle, a integração exata do gradiente de um polinômio $\phi$ na mesma é feita pela fórmula

$$
\oint_{\partial \Omega} \nabla \phi(x, y) \cdot n d s=\sum_{k=1}^{\mathrm{NPG}}\left[\phi_{x}\left(x_{g}^{(k)}, y_{g}^{(k)}\right) n_{x}^{(k)}+\phi_{y}\left(x_{g}^{(k)}, y_{g}^{(k)}\right) n_{y}^{(k)}\right] w_{g}^{(k)}
$$

onde NPG são o número de pontos de Gauss. 
Considerando $P_{a}\left(x_{a}, y_{a}\right), P_{b}\left(x_{b}, y_{b}\right)$ e $P_{c}\left(x_{c}, y_{c}\right)$ os pontos da figura em 2.10, segue:

$$
\begin{aligned}
& L_{1}=\left|P_{a}-P_{b}\right|=\sqrt{\left(x_{b}-x_{a}\right)^{2}+\left(y_{b}-y_{a}\right)^{2}} \\
& L_{2}=\left|P_{b}-P_{c}\right|=\sqrt{\left(x_{c}-x_{b}\right)^{2}+\left(y_{c}-y_{b}\right)^{2}}
\end{aligned}
$$

As tabelas 2.2, 2.3 e 2.4 apresentam, respectivamente, para uma dada face de um volume de controle, as coordenadas dos pontos de Gauss, dos pesos e nas componentes das normais.

\begin{tabular}{|c|c|c|c|}
\hline & $P(x, y)$ & $W$ & $\hat{n}$ \\
\hline $\mathrm{G}$ & $\frac{0.5\left[L_{1}\left(P_{a}+P_{b}\right)+L_{2}\left(P_{b}+P_{c}\right)\right]}{L_{1}+L_{2}}$ & $\left|P_{a}-P_{c}\right|$ & $\frac{\left(y_{c}-y_{a}, x_{a}-x_{c}\right)}{W_{g}}$ \\
\hline
\end{tabular}

Tabela 2.2: pontos de Gauss, pesos e normais para reconstrução de segunda ordem

\begin{tabular}{|c|c|c|c|}
\hline & $P(x, y)$ & $W$ & $\hat{n}$ \\
\hline $\mathrm{G}_{1}$ & $0.5\left(P_{a}+P_{b}\right)$ & $L_{1}$ & $L_{1}^{-1}\left(y_{b}-y_{a}, x_{a}-x_{b}\right)$ \\
\hline $\mathrm{G}_{2}$ & $0.5\left(P_{b}+P_{c}\right)$ & $L_{2}$ & $L_{2}^{-1}\left(y_{c}-y_{b}, x_{b}-x_{c}\right)$ \\
\hline
\end{tabular}

Tabela 2.3: pontos de Gauss, pesos e normais para reconstrução de terceira ordem

\begin{tabular}{|c|c|c|c|}
\hline & $P(x, y)$ & $W$ & $\hat{n}$ \\
\hline $\mathrm{G}_{1}$ & $0.5\left(P_{a}+P_{b}\right)+(2 \sqrt{3})^{-1}\left(P_{a}-P_{b}\right)$ & $0.5 L_{1}$ & $L_{1}^{-1}\left(y_{b}-y_{a}, x_{a}-x_{b}\right)$ \\
\hline $\mathrm{G}_{2}$ & $0.5\left(P_{a}+P_{b}\right)-(2 \sqrt{3})^{-1}\left(P_{a}-P_{b}\right)$ & $0.5 L_{2}$ & $L_{2}^{-1}\left(y_{c}-y_{b}, x_{b}-x_{c}\right)$ \\
\hline $\mathrm{G}_{3}$ & $0.5\left(P_{b}+P_{c}\right)+(2 \sqrt{3})^{-1}\left(P_{b}-P_{c}\right)$ & $0.5 L_{1}$ & $L_{1}^{-1}\left(y_{b}-y_{a}, x_{a}-x_{b}\right)$ \\
\hline $\mathrm{G}_{4}$ & $0.5\left(P_{b}+P_{c}\right)-(2 \sqrt{3})^{-1}\left(P_{b}-P_{c}\right)$ & $0.5 L_{2}$ & $L_{2}^{-1}\left(y_{c}-y_{b}, x_{b}-x_{c}\right)$ \\
\hline
\end{tabular}

Tabela 2.4: pontos de Gauss, pesos e normais para reconstrução de quarta ordem

Para calcular integrais de área de triângulos, adotou-se as técnicas de quadratura gaussianas apresentadas por Dunavant [12]. Assim sendo, um dado triângulo, para calcular essas integrais seguiu-se a fórmula

$$
\iint_{\Omega} \phi(x, y) d \Omega=\sum_{k=1}^{\mathrm{NPG}} \mathrm{A}_{\mathrm{T}} w_{k} \phi\left(x_{g}^{(k)} x_{A}+y_{g}^{(k)} x_{B}+z_{g}^{(k)} x_{C}, x_{g}^{(k)} y_{A}+y_{g}^{(k)} y_{B}+z_{g}^{(k)} y_{C}\right)
$$

- $A_{T}$ é a área do triângulo onde se calcula a integral.

- $\left(x_{A}, y_{A}\right),\left(x_{B}, y_{B}\right)$ e $\left(x_{C}, y_{C}\right)$ são as coordenadas dos vértices do triângulo. 
- $w_{g}^{(k)}$ são pesos para os pontos de Gauss.

- $x_{g}^{(k)}, y_{g}^{(k)}$ e $z_{g}^{(k)}$ são parâmetros para determinar os pontos de Gauss a partir das coordenadas dos vértices.

Os pesos e pontos de Gauss podem ser obtidos no mesmo artigo [12].

Exemplos de fórmulas de integração, via quadratura gaussiana, de área de triângulos, obtido utilizando [12] e apresentado em [52], são dadas por

$-\iint_{\Omega} \phi(x, y) d \Omega=\frac{\mathrm{A}}{3} \sum_{k=1}^{3} \phi\left(x_{m}^{(k)}, y_{m}^{(m)}\right)$
- $\iint_{\Omega} \phi(x, y) d \Omega=\frac{\mathrm{A}}{60}\left[3 \sum_{k=1}^{3} \phi\left(x_{v}^{(k)}, y_{v}^{(k)}\right)+8 \sum_{k=1}^{3} \phi\left(x_{m}^{(k)}, y_{m}^{(m)}\right)+27 f\left(x_{c}, y_{c}\right)\right]$

onde $\left(x_{v}^{(k)}, y_{v}^{(k)}\right)$ e $\left(x_{m}^{(k)}, y_{m}^{(k)}\right)$ são, respectivamente, os vértices do triângulo e os pontos médios dos lados do mesmo. O ponto $\left(x_{c}, y_{c}\right)$ é o centróide do triângulo, dado por

$$
x_{c}=\frac{1}{3} \sum_{k=1}^{3} x_{v}^{(k)} \quad y_{c}=\frac{1}{3} \sum_{k=1}^{3} y_{v}^{(k)}
$$

Essas duas últimas fórmulas de integração de área tem precisão, respectivamente, de ordens 2 e 3.

As três seções a seguir detalham o tratamento da reconstrução na fronteira para os três tipos de condições de fronteira: Dirichlet, Neumann e Robin. Em essência, a idéia básica, para as três condições de fronteira citadas, consiste em forçar a reconstrução no contorno adicionando mais restrições na formação do sistema, além a restrição da equação de conservação da média. Tais restrições são adicionadas de tal modo que as reconstruções sejam satisfeitas, bem ajustadas no sentido dos mínimos quadrados, nos pontos de Gauss dos volumes de controle que estão sobre a fronteira. Segue os detalhamentos.

\subsubsection{Condição de fronteira de Dirichlet}

Como comentado, para garantir que a ordem seja preservada no contorno, é adicionado, além da equação de conservação da média, mais restrições na formação do sistema. As equações de restrição adicionais são aplicadas nos pontos de Gauss da fronteira. A quantidade de pontos necessários vai depender da ordem de 
reconstrução. A tabela 2.5, obtida em [2], apresenta a relação entre a ordem de reconstrução e o número de pontos de Gauss. A figura 2.11, obtida também em [2], ilustra o posicionamento dos pontos de Gauss na fronteira, para os volumes de controle cell-vertex.

\begin{tabular}{|c|c|}
\hline ordem & $\mathrm{n}$ - de pontos de Gauss \\
\hline 2 & 3 \\
\hline 3 & 3 \\
\hline 4 & 5 \\
\hline
\end{tabular}

Tabela 2.5: Número de pontos de Gauss necessários para cada ordem de reconstrução

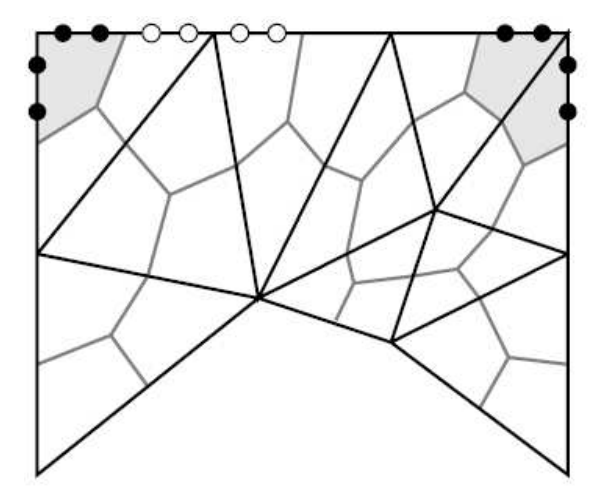

Figura 2.11: pontos de Gauss - cell-vertex

Para ilustrar, segue a apresentação dos sistemas envolvidos nos casos de reconstrução de segunda e terceira ordens.

- Reconstrução de 2a ordem:

$$
\left[\begin{array}{ccc}
1 & \bar{x}_{i} & \bar{y}_{i} \\
1 & \Delta x_{1} & \Delta y_{1} \\
1 & \Delta x_{2} & \Delta y_{2} \\
\hline w_{i 1} & w_{i 1} \widehat{x}_{i 1} & w_{i 1} \widehat{y}_{i 1} \\
w_{i 2} & w_{i 2} \widehat{x}_{i 2} & w_{i 2} \widehat{y}_{i 2} \\
w_{i 3} & w_{i 3} \widehat{x}_{i 3} & w_{i 3} \widehat{y}_{i 3} \\
\vdots & \vdots & \vdots \\
w_{i N} & w_{i N} \widehat{x}_{i N} & w_{i N} \widehat{y}_{i N}
\end{array}\right]\left[\begin{array}{c}
\phi \\
\phi_{x} \\
\phi_{y} \\
\frac{1}{2} \phi_{x x} \\
\phi_{x y} \\
\frac{1}{2} \phi_{y y} \\
\vdots
\end{array}\right]=\left[\begin{array}{c}
\bar{\phi}_{i} \\
u_{1} \\
u_{2} \\
\hline w_{i 1} \bar{\phi}_{1} \\
w_{i 2} \bar{\phi}_{2} \\
w_{i 3} \bar{\phi}_{3} \\
\vdots \\
w_{i N} \bar{\phi}_{N}
\end{array}\right]
$$

- Reconstrução de 3a ordem: 


$$
\left[\begin{array}{cccccc}
1 & \bar{x}_{i} & \bar{y}_{i} & \bar{x}^{2} & \overline{x y}_{i} & \bar{y}^{2} \\
1 & \Delta x_{1} & \Delta y_{1} & \Delta^{2} x_{1} & \Delta x_{1} \Delta y_{1} & \Delta^{2} y_{1} \\
1 & \Delta x_{2} & \Delta y_{2} & \Delta^{2} x_{2} & \Delta x_{2} \Delta y_{2} & \Delta^{2} y_{2} \\
\hline w_{i 1} & w_{i 1} \widehat{x}_{i 1} & w_{i 1} \widehat{y}_{i 1} & w_{i 1} \widehat{x}^{2}{ }_{i 1} & w_{i 1} \widehat{x y}_{i 1} & w_{i 1} \widehat{y}^{2}{ }^{2} \\
w_{i 2} & w_{i 2} \widehat{x}_{i 2} & w_{i 2} \widehat{y}_{i 2} & w_{i 2} \widehat{x}^{2}{ }_{i 2} & w_{i 2} \widehat{x y}_{i 2} & w_{i 2} \widehat{y}^{2}{ }_{i 2} \\
w_{i 3} & w_{i 3} \widehat{x}_{i 3} & w_{i 3} \widehat{y}_{i 3} & w_{i 3} \widehat{x}^{2}{ }_{i 3} & w_{i 3} \widehat{x y}_{i 3} & w_{i 3} \widehat{y}^{2}{ }_{i 3} \\
\vdots & \vdots & \vdots & \vdots & \vdots & \vdots \\
w_{i N} & w_{i N} \widehat{x}_{i N} & w_{i N} \widehat{y}_{i N} & w_{i N} \widehat{x}^{2}{ }_{i N} & w_{i N} \widehat{x y}_{i N} & w_{i N}{\widehat{y^{2}}}_{i N}
\end{array}\right]\left[\begin{array}{c}
\phi \\
\phi_{x} \\
\phi_{y} \\
\frac{1}{2} \phi_{x x} \\
\phi_{x y} \\
\frac{1}{2} \phi_{y y} \\
\vdots
\end{array}\right]=\left[\begin{array}{c}
\bar{\phi}_{i} \\
u_{1} \\
u_{2} \\
\hline w_{i 1} \bar{\phi}_{1} \\
w_{i 2} \bar{\phi}_{2} \\
w_{i 3} \bar{\phi}_{3} \\
\vdots \\
w_{i N} \bar{\phi}_{N}
\end{array}\right]
$$

onde, para um dado volume de controle $i, \Delta^{m} x_{k} \Delta^{n} y_{k}=\left(x_{g}^{(k)}-x_{i}\right)^{m}\left(y_{g}^{(k)}-y_{i}\right)^{n}$ e $u_{k}=u\left(x_{g}^{(k)}, y_{g}^{(k)}\right)$, com $k=1, \ldots, 4$. No vetor dos termos independentes, $u_{1}$ e $u_{2}$ são os valores da solução na fronteira, os quais são conhecidos no caso da condição de Dirichlet.

Reconstrução de quarta de ordem é apenas uma extensão da estrutura de segunda e terceiras ordens, com o acréscimo no número de restrições na fronteira. Para quarta ordem, temos 10 colunas e 5 restrições, sendo 4 dessas ( da segunda a quarta linha do sistema) aplicadas no ponto de gauss. O número de colunas é igual ao número de termos necessários para fazer a reconstrução, uma vez que as soluções dos sistemas fornecem as derivadas necessárias para a aproximação polinomial.

Um tratamento, baseado em eliminação gaussiana, conforme Van Altena [2], também deve ser feito sobre as equações de restrição, incluindo a primeira equação, equação associada a conservação da média. 0 processo de eliminação deve seguir os passos abaixo:

1. Determine o maior coeficiente, em módulo, da $i$-ésima linha e divida todos os coeficiente dessa linha por esse coeficiente, incluindo o lado direito do sistema.

2. Troque a coluna que contém o maior coeficiente com a coluna que contém o pivô. Ao fazer essa troca, as linhas do vetor solução também tem que serem trocadas.

3. Realize a eliminação gaussiana nas linhas abaixo do pivô (da linha $i+1$ até a linha $N$ ).

Essa estratégia tem que ser feita apenas nas linhas de restrição. Feita a eliminação, aplica-se a transformação de Householder e em seguida resolve-se o sistema fazendo substituição regressiva. Essa técnica também deve ser empregada nas fronteiras sob condição de Neumann e Robin. 


\subsubsection{Condições de fronteira de Neumann}

A idéia para condição de fronteiras sob condição de Neumann é análoga ao caso de Dirichlet, com a diferença de que o que deve ser forçado no contorno, é que os polinômios de reconstrução nos pontos de Gauss se ajustem as derivadas da função incógnita nos pontos de Gauss. Novamente, para ilustrar, segue a apresentação dos sistemas envolvido nos casos de reconstrução de segunda e terceira ordens.

- Reconstrução de 2a ordem:

$$
\left[\begin{array}{ccc}
1 & \bar{x}_{i} & \bar{y}_{i} \\
0 & n_{x_{1}} & n_{y_{1}} \\
0 & n_{x_{2}} & n_{y_{2}} \\
\hline w_{i 1} & w_{i 1} \widehat{x}_{i 1} & w_{i 1} \widehat{y}_{i 1} \\
w_{i 2} & w_{i 2} \widehat{x}_{i 2} & w_{i 2} \widehat{y}_{i 2} \\
w_{i 3} & w_{i 3} \widehat{x}_{i 3} & w_{i 3} \widehat{y}_{i 3} \\
\vdots & \vdots & \vdots \\
w_{i N} & w_{i N} \widehat{x}_{i N} & w_{i N} \widehat{y}_{i N}
\end{array}\right]\left[\begin{array}{c}
\phi \\
\phi_{x} \\
\phi_{y} \\
\frac{1}{2} \phi_{x x} \\
\phi_{x y} \\
\frac{1}{2} \phi_{y y} \\
\vdots
\end{array}\right]=\left[\begin{array}{c}
\bar{\phi}_{i} \\
u_{1}^{\prime} \\
u_{2}^{\prime} \\
\hline w_{i 1} \bar{\phi}_{1} \\
w_{i 2} \bar{\phi}_{2} \\
w_{i 3} \bar{\phi}_{3} \\
\vdots \\
w_{i N} \bar{\phi}_{N}
\end{array}\right]
$$

- Reconstrução de 3a ordem:

$$
\left[\begin{array}{cccccc}
1 & \bar{x}_{i} & \bar{y}_{i} & \bar{x}^{2} & \overline{x y}_{i} & \bar{y}^{2} \\
0 & n_{x_{1}} & n_{y_{1}} & 2 n_{x_{1}} \Delta x_{1} & n_{x_{1}} \Delta y_{1}+n_{y_{1}} \Delta x_{1} & 2 n_{y_{1}} \Delta y_{1} \\
0 & n_{x_{2}} & n_{y_{2}} & 2 n_{x_{2}} \Delta x_{2} & n_{x_{2}} \Delta y_{2}+n_{y_{2}} \Delta x_{2} & 2 n_{y_{1}} \Delta y_{2} \\
\hline w_{i 1} & w_{i 1} \widehat{x}_{i 1} & w_{i 1} \widehat{y}_{i 1} & w_{i 1} \widehat{x}^{2}{ }_{i 1} & w_{i 1} \widehat{x y}_{i 1} & w_{i 1} \widehat{y}^{2}{ }_{i 1} \\
w_{i 2} & w_{i 2} \widehat{x}_{i 2} & w_{i 2} \widehat{y}_{i 2} & w_{i 2} \widehat{x}^{2}{ }_{i 2} & w_{i 2} \widehat{x y}_{i 2} & w_{i 2} \widehat{y}^{2}{ }_{i 2} \\
w_{i 3} & w_{i 3} \widehat{x}_{i 3} & w_{i 3} \widehat{y}_{i 3} & w_{i 3} \widehat{x}^{2} & w_{i 3} \widehat{x y}_{i 3} & w_{i 3} \widehat{y}^{2}{ }_{i 3} \\
\vdots & \vdots & \vdots & \vdots & \vdots & \vdots \\
w_{i N} & w_{i N} \widehat{x}_{i N} & w_{i N} \widehat{y}_{i N} & w_{i N} \widehat{x}^{2}{ }_{i N} & w_{i N} \widehat{x y}_{i N} & w_{i N}{\widehat{y^{2}}}_{i N}
\end{array}\right]\left[\begin{array}{c}
\phi \\
\phi_{x} \\
\phi_{y} \\
\frac{1}{2} \phi_{x x} \\
\phi_{x y} \\
\frac{1}{2} \phi_{y y} \\
\vdots
\end{array}\right]=\left[\begin{array}{c}
\bar{\phi}_{i} \\
u_{1}^{\prime} \\
u_{2}^{\prime} \\
\hline w_{i 1} \bar{\phi}_{1} \\
w_{i 2} \bar{\phi}_{2} \\
w_{i 3} \bar{\phi}_{3} \\
\vdots \\
w_{i N} \bar{\phi}_{N}
\end{array}\right]
$$

onde, para um dado volume de controle $i, \Delta^{m} x_{k} \Delta^{n} y_{k}=\left(x_{g}^{(k)}-x_{i}\right)^{m}\left(y_{g}^{(k)}-y_{i}\right)^{n},\left(n_{x_{k}}, n_{y_{k}}\right)$ as normais no ponto de gauss e $u_{k}^{\prime}=\nabla u\left(x_{g}^{(k)}, y_{g}^{(k)}\right)$, com $k=1,2$. No vetor dos termos independentes, $u_{1}^{\prime}$ e $u_{2}^{\prime}$ são os valores das derivadas em relação a normal à fronteira, as quais são conhecidas no caso da condição de Neumann. 
A reconstrução de quarta ordem segue a mesma idéia com relação a condição de fronteira de Dirichlet. Os sistemas nesses casos são sistemas com 10 colunas tendo o acréscimo de 4 restrições (da segunda a quarta linha) nos pontos de Gauss.

\subsection{Condição de fronteira de Robin}

A condição de fronteira de Robin é dada por

$$
\alpha \phi+\beta \nabla \phi \cdot n=g
$$

onde $\alpha$ e $\beta$ são constantes.

A reconstrução para um dado volume de controle $i$ é dado por

$$
\begin{aligned}
& \phi^{\mathrm{R}}(x, y)=\phi+\phi_{x}\left(x-x_{i}\right)+\phi_{y}\left(y-y_{i}\right)+\frac{1}{2} \phi_{x x}\left(x-x_{i}\right)^{2}+ \\
& \phi_{x y}\left(x-x_{i}\right)\left(y-y_{i}\right)+\frac{1}{2} \phi_{y y}\left(y-y_{i}\right)^{2}+\frac{1}{6} \phi_{x x x}\left(x-x_{i}\right)^{3}+\ldots
\end{aligned}
$$

Derivando em relação a $x$ e a $y$,

$$
\left\{\begin{array}{l}
\phi_{x}^{\mathrm{R}}(x, y)=\phi_{x}+\phi_{x x}\left(x-x_{i}\right)+\phi_{x y}\left(y-y_{i}\right)+\frac{1}{2} \phi_{x x x}\left(x-x_{i}\right)^{2}+\ldots \\
\phi_{y}^{\mathrm{R}}(x, y)=\phi_{y}+\phi_{x y}\left(x-x_{i}\right)+\phi_{y y}\left(y-y_{i}\right)+\ldots
\end{array}\right.
$$

Substituindo (2.12) e (2.13) em (2.11), tem-se

$$
\begin{array}{r}
\alpha\left[\phi+\phi_{x}\left(x-x_{i}\right)+\phi_{y}\left(y-y_{i}\right)+\frac{1}{2} \phi_{x x}\left(x-x_{i}\right)^{2}+\phi_{x y}\left(x-x_{i}\right)\left(y-y_{i}\right)+\frac{1}{2} \phi_{y y}\left(y-y_{i}\right)^{2}+\ldots\right]+ \\
\beta\left[\left(\phi_{x}+\phi_{x x}\left(x-x_{i}\right)+\phi_{x y}\left(y-y_{i}\right)+\frac{1}{2} \phi_{x x x}\left(x-x_{i}\right)^{2}+\ldots\right) n_{x}+\right. \\
\left.\left(\phi_{y}+\phi_{x y}\left(x-x_{i}\right)+\phi_{y y}\left(y-y_{i}\right)+\ldots\right) n_{y}\right]=g
\end{array}
$$


A seguir tem-se um exemplo de como ficaria o sistema para o caso de reconstrução de segunda ordem

$$
\left[\begin{array}{ccc}
1 & \bar{x}_{i} & \bar{y}_{i} \\
\alpha & \alpha \Delta x_{1}+\beta n_{x_{1}} & \alpha \Delta y_{1}+\beta n_{y_{1}} \\
\alpha & \alpha \Delta x_{2}+\beta n_{x_{2}} & \alpha \Delta y_{2}+\beta n_{y_{2}} \\
\hline w_{i 1} & w_{i 1} \widehat{x}_{i 1} & w_{i 1} \widehat{y}_{i 1} \\
w_{i 2} & w_{i 2} \widehat{x}_{i 2} & w_{i 2} \widehat{y}_{i 2} \\
w_{i 3} & w_{i 3} \widehat{x}_{i 3} & w_{i 3} \widehat{y}_{i 3} \\
\vdots & \vdots & \vdots \\
w_{i N} & w_{i N} \widehat{x}_{i N} & w_{i N} \widehat{y}_{i N}
\end{array}\right]\left[\begin{array}{c}
\phi \\
\phi_{x} \\
\phi_{y} \\
\frac{1}{2} \phi_{x x} \\
\phi_{x y} \\
\frac{1}{2} \phi_{y y} \\
\vdots
\end{array}\right]=\left[\begin{array}{c}
\bar{\phi}_{i} \\
g_{1} \\
g_{2} \\
\hline w_{i 1} \bar{\phi}_{1} \\
w_{i 2} \bar{\phi}_{2} \\
w_{i 3} \bar{\phi}_{3} \\
\vdots \\
w_{i N} \bar{\phi}_{N}
\end{array}\right]
$$

onde $\Delta^{m} x_{k} \Delta^{n} y_{k}=\left(x_{g}^{(k)}-x_{i}\right)^{m}\left(y_{g}^{(k)}-y_{i}\right)^{n},\left(n_{x_{k}}, n_{y_{k}}\right)$ as normais no ponto de gauss. No vetor dos termos independentes, $g_{1}$ e $g_{2}$ são os valores da combinação $\alpha \phi+\beta \nabla \phi \cdot n$, os quais são conhecidos na fronteira sob condição de Robin.

Novamente, para terceiras e quartas ordens, o método de construção é o mesmo, diferindo apenas no número de colunas e restrições das equações nos pontos de Gauss. Para terceira ordem temos 6 colunas e 3 restrições como no caso de segunda ordem. No caso de quarta ordem, temos 10 colunas e 5 restrições.

\subsection{Resolução da EDP}

Considere a equação de advecção-difusão com termo fonte

$$
\nabla \cdot \vec{\omega} \phi-\nabla \cdot(\kappa \nabla \phi)=f
$$

A equação (2.15) não varia com o tempo e depende apenas de variáveis espaciais $x$ e $y$. Desse modo, se adicionarmos $\phi_{t}$ no primeiro membro de (2.15), a relação de igualdade vai permanecer, uma vez que $\phi_{t}$ é zero.

$$
\frac{\partial \phi}{\partial t}+\nabla \cdot \vec{\omega} \phi-\nabla \cdot(\kappa \nabla \phi)=f
$$

Integrando o termo transiente, os fluxos advectivos e difusivos e o termo fonte em (2.16), em cada volume 
de controle do domínio discretizado, segue

$$
\iint_{\Omega} \frac{\partial \phi}{\partial t} d \Omega+\iint_{\Omega}[\nabla \cdot \vec{\omega} \phi-\nabla \cdot(\kappa \nabla \phi)] d \Omega=\iint_{\Omega} f d \Omega
$$

Aplicando o Teorema da divergência de Gauss,

$$
\iint_{\Omega} \frac{\partial \phi}{\partial t} d \Omega+\oint_{\partial \Omega}[\vec{\omega} \phi-\kappa \nabla \phi] \cdot n d s=\iint_{\Omega} f d \Omega .
$$

O termo transiente pode ser aproximado considerando o seu valor médio no volume de controle, daí

$$
\frac{\partial \bar{\phi}}{\partial t} A_{\Omega}+\oint_{\partial \Omega}[\vec{\omega} \phi-\kappa \nabla \phi] d \Omega=\iint_{\Omega} f d \Omega
$$

Aproximando por diferença progressiva o termo temporal,

$$
\begin{gathered}
\frac{\bar{\phi}^{(n+1)}-\bar{\phi}^{(n)}}{\Delta t} A_{\Omega}+\oint_{\partial \Omega}[\vec{\omega} \phi-\kappa \nabla \phi] d \Omega=\iint_{\Omega} f d \Omega . \\
\bar{\phi}^{(n+1)}=\bar{\phi}^{(n)}-\frac{\alpha_{k} \Delta t}{A_{\Omega}}[\underbrace{\oint_{\partial \Omega}[\vec{\omega} \phi-\kappa \nabla \phi] d \Omega}_{\mathrm{IF}}-\underbrace{\iint_{\Omega} f d \Omega}_{\mathrm{TF}}] \quad k=1,2, \ldots, n p
\end{gathered}
$$

onde $n p$ é o número de estágios e $\alpha_{k}$ são parâmetros de Runge-Kutta.

Perceba que no desenvolvimento a derivada de $\phi$ em relação ao tempo acrescentada tem uma função auxiliar no processo de obtenção da solução numérica. Foi acrescentada com a finalidade de se montar um processo de integração temporal. O método de Runge-Kutta multiestágio é um método de evolução temporal. Assim sendo, por ser um problema pseudotransiente, a integração temporal deverá ser realizada até atingir um estado estacionário. Dessa forma, a partir de uma solução inicial, a solução irá evoluir no tempo, e em cada iteração do processo de integração temporal, a solução será reconstruída com as aproximações nodais 
obtidas em cada volume de controle. Essas reconstruções, que são polinômios, são então utilizadas para calcular as integrais dos fluxos. Os passos são realizados até que, como comentado anteriormente, a solução fique estacionária em cada volume de controle.

Os parâmetros $\alpha_{k}$ em (2.21) são calibrados de acordo com o problema e na literatura existem vários que podem ser utilizados. Em Lindquist [41], temos 4 estágios onde $\alpha_{1}=1 / 4, \alpha_{2}=1 / 3, \alpha_{3}=1 / 2$ e $\alpha_{4}=1$. Em Bijan [44] tem-se $\alpha_{1}=0.11, \alpha_{2}=0.2766, \alpha_{3}=0.5$ e $\alpha_{4}=1$. Em Gooch [28], para 5 estágios tem-se por exemplo $\alpha_{1}=1 / 15, \alpha_{2}=7 / 45, \alpha_{3}=2 / 7, \alpha_{4}=1 / 2$ e $\alpha_{5}=1$. No presente trabalho utilizaram-se esses últimos parâmetros.

O termo IF é a integral dos fluxos, englobando os fluxos advectivos e os fluxos difusivos, e envolve o cálculo de uma integral de linha em torno do volume de controle. O contorno de um dado volume de controle faz comunicação, tem intersecção, com outros volumes de controle. Assim sendo, dado um volume de controle I que tem uma face em comum com um volume de controle J (figura 2.12), para calcular a integral na face ABC, faz-se três considerações:

- Pontos de Gauss: Os pontos de Gauss são utilizados nesse calculo e o número deles necessário depende da ordem do polinômio de reconstrução. Os polinômios são avaliados nos pontos de Gauss que se localizam na face $A B C$ ao realizar a integração.

- Fluxos advectivos: O fluxo advectivo é dado pelo termo $\vec{\omega} \phi$. Para calculá-lo na face ABC leva-se em consideração a direção do escoamento nos pontos de Gauss. Isso caracteriza um esquema upwind e associa propriedades físicas do escoamento com o modelo matemático representado pela equação de advecção-difusão. Nesse trabalho, a direção do escoamento foi obtida tomando por base o sinal produto interno $\omega \cdot n_{g}$, onde $\omega$ é o vetor velocidade de advecção e $n_{g}$ é o vetor normal a face ABC no ponto de Gauss. Se para um dado ponto de gauss o escoamento está na direção de I para J, então o polinômio de reconstrução associado ao volume de controle I é utilizado para avaliar $\phi$ no ponto de gauss na hora de realizar a integração.

- Fluxos difusivos: Os fluxos difusivos nos pontos de Gauss da face ABC são calculados tomando as médias ponderadas pelas áreas dos volumes de controle [2] $\phi_{\mathrm{I}}$ e $\phi_{\mathrm{J}}$ em cada ponto de Gauss.

O termo fonte TF pode ser calculado usando o método (2.10).

Assim sendo, dado um volume de controle I, o cálculo de IF é feito usando a fórmula 


$$
\mathrm{IF}=\sum_{i=1}^{\mathrm{NF}} \sum_{k=1}^{\mathrm{NPG}}\left[\vec{\omega}_{g}^{(k)} \phi^{\mathrm{R}}\left(x_{g}^{(k)}, y_{g}^{(k)}\right)-\kappa\left(x_{g}^{(k)}, y_{g}^{(k)}\right) \nabla \phi_{m}^{\mathrm{R}}\left(x_{g}^{(k)}, y_{g}^{(k)}\right)\right] \cdot n_{g}^{(k)}
$$

onde

- NF e NPG são, recpectivamente, o número de faces do volume de controle e o número de pontos de Gauss em cada face.

- $\left(x_{g}^{(k)}, y_{g}^{(k)}\right)$ são os pontos de Gauss em uma dada face ABC que está na intersecção de um volume de controle I com outro J.

- $n_{g}=\left(n_{x}, n_{y}\right)$ são as componentes das normais nos pontos de Gauss.

- Velocidade de advecção avaliada nos pontos de Gauss: $w_{g}=\left(u\left(x_{g}^{(k)}, y_{g}^{(k)}\right), v\left(x_{g}^{(k)}, y_{g}^{(k)}\right)\right)$

- Média do gradiente na face:

$$
\nabla \phi_{m}^{\mathrm{R}}\left(x_{g}^{(k)}, y_{g}^{(k)}\right)=\frac{\kappa_{\mathbf{l}}\left(x_{g}^{(k)}, y_{g}^{(k)}\right) \nabla_{\mathbf{l}} \phi^{\mathrm{R}}\left(x_{g}^{(k)}, y_{g}^{(k)}\right) \mathrm{AVC}_{\mathbf{I}}+\kappa_{\mathrm{J}}\left(x_{g}^{(k)}, y_{g}^{(k)}\right) \nabla_{\mathrm{J}} \phi^{\mathrm{R}}\left(x_{g}^{(k)}, y_{g}^{(k)}\right) \mathrm{AVC}_{\mathbf{J}}}{\mathrm{AVC}_{\mathbf{I}}+\mathrm{AVC}_{\mathbf{J}}}
$$

onde $A V C_{1}$ e $A V C_{J}$ são as áreas dos volumes de controle I e J, volumes de controle com a face $A B C$ em comum.

\subsection{Validação}

A metodologia para validação do método de reconstrução de alta ordem segue a que foi adotada por Van Altena [2]. Essa metodologia se baseia na análise na ordem de precisão da integral dos fluxos convectivos e difusivos que aparecem na equação de advecção-difusão

$$
\mathrm{R}=\iint_{\Omega}[\nabla \cdot(\vec{\omega} \phi)-\nabla \cdot(\kappa \nabla \phi)] d \Omega
$$

considerando o comportamento sobre três tipos de condições de fronteira: Dirichlet, Neumann e Robin. Nesse processo, uma seqüência de integrais são calculadas, utilizando os polinômios de reconstrução, sobre malhas não-estruturadas que aumentam sucessivamente o grau de refinamento. 


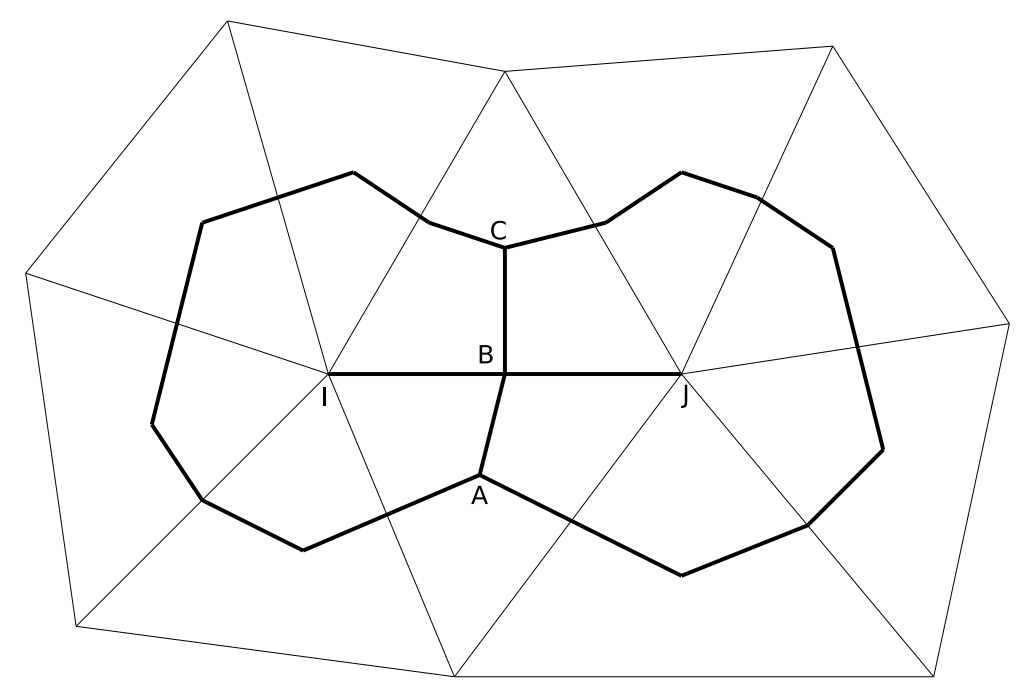

Figura 2.12: Ilustração para o cálculo da integral de linha dos fluxos

Em toda análise de ordem o cálculos de normas são necessárias. Assim sendo, três tipos de normas foram consideradas: $L_{\infty}, L_{1}$ e $L_{2}$. A norma $L_{\infty}$ é simplesmente o maior erro em magnitude (maior erro em valor absoluto) na malha, ou seja, $\mathrm{L}_{\infty}=\max _{1 \leq i \leq \mathrm{N}}\left|E_{i}\right|$ onde $\mathrm{E}_{i}=\left|\mathrm{R}_{\mathrm{ex}}-\mathrm{R}_{\mathrm{ap}}\right|$, sendo $\mathrm{R}_{\mathrm{ex}}$ a integral exata dos fluxos $\mathrm{e}$ $\mathrm{R}_{\mathrm{ap}}$ a integral aproximada. As demais normas são calculadas usando

$$
\mathrm{L}_{k}=\left[\frac{\sum_{i=1}^{\mathrm{N}} \mathrm{A}_{i} \mathrm{E}_{i}}{\sum_{i=1}^{\mathrm{N}} \mathrm{A}_{i}}\right]^{\frac{1}{k}} \quad i=1, \ldots, \mathrm{N}
$$

onde $\mathrm{N}$ é o número de volumes de controle e $\mathrm{A}_{i}$ é a área do volume de controle $i$.

A ordem de precisão é determinada plotando o número de volumes de controle pela norma do erro em um gráfico em escala logarítmica. Feito isso, segue-se uma regressão linear dos pontos no gráfico. O coeficiente angular da reta de ajuste é negativo e o dobro de seu valor em módulo irá fornecer a ordem de precisão.

Durante o desenvolvimento do trabalho, ao aplicar as estratégias de pivoteamento com vias ao aprimoramento da reconstrução, apresentadas por Van Althena [2], em fronteiras retas sob condição de Neumann para reconstrução de segunda e quarta ordem, observa-se um cancelamento de termos. Ao aplicar a estratégia de eliminação gaussiana, por exemplo, para reconstrução de segunda ordem, o sistema linear acabava anulando uma linha inteira. 


$$
\left[\begin{array}{ccc}
1 & \bar{x}_{i} & \bar{y}_{i} \\
0 & n_{x_{1}} & n_{y_{1}} \\
0 & n_{x_{2}} & n_{y_{2}} \\
\hline w_{i 1} & w_{i 1} \widehat{x}_{i 1} & w_{i 1} \widehat{y}_{i 1} \\
w_{i 2} & w_{i 2} \widehat{x}_{i 2} & w_{i 2} \widehat{y}_{i 2} \\
w_{i 3} & w_{i 3} \widehat{x}_{i 3} & w_{i 3} \widehat{y}_{i 3} \\
\vdots & \vdots & \vdots \\
w_{i N} & w_{i N} \widehat{x}_{i N} & w_{i N} \widehat{y}_{i N}
\end{array}\right]\left[\begin{array}{c}
\phi \\
\phi_{x} \\
\phi_{y} \\
\frac{1}{2} \phi_{x x} \\
\phi_{x y} \\
\frac{1}{2} \phi_{y y} \\
\vdots
\end{array}\right]=\left[\begin{array}{c}
\bar{\phi}_{i} \\
u_{1}^{\prime} \\
u_{2}^{\prime} \\
\hline w_{i 1} \bar{\phi}_{1} \\
w_{i 2} \bar{\phi}_{2} \\
w_{i 3} \bar{\phi}_{3} \\
\vdots \\
w_{i N} \bar{\phi}_{N}
\end{array}\right]
$$

Na fronteira de um quadrado unitário ao longo de $x=1$, fronteira reta, os vetores normais $\left(n_{x_{1}}, n_{y_{1}}\right)$, $\left(n_{x_{2}}, n_{y_{2}}\right)$ são unitários e tem a mesma direção e sentido, logo são iguais. Quando se aplica então a eliminação gaussiana, a terceira linha acaba por se anular.

$$
\left[\begin{array}{ccc}
1 & \bar{x}_{i} & \bar{y}_{i} \\
0 & 1 & 0 \\
0 & 0 & 0 \\
\hline 0 & 0 & d_{43} \\
0 & 0 & d_{53} \\
0 & 0 & d_{63} \\
\vdots & \vdots & \vdots \\
0 & 0 & d_{i N}
\end{array}\right]\left[\begin{array}{c}
\phi \\
\phi_{x} \\
\phi_{y} \\
\frac{1}{2} \phi_{x x} \\
\phi_{x y} \\
\frac{1}{2} \phi_{y y} \\
\vdots
\end{array}\right]=\left[\begin{array}{c}
\bar{\phi}_{i} \\
b_{2} \\
b_{3} \\
\hline b_{4} \\
b_{5} \\
b_{6} \\
\vdots \\
b_{N}
\end{array}\right]
$$

E isso ocorre para todo volume de controle na fronteira do quadrado unitário sob condição de Neumann. Em reconstruções de terceira ordem isso não ocorre. Assim sendo, para contornar esse problema, nos casos de reconstrução de segunda e quarta ordem, nas fronteiras sob condição de Neumann, fazia-se reconstrução de terceira ordem.

Para efeito de comparação e avaliação do erro, foi tomada como sendo a integral exata $R_{\text {ex }}$ a integral calculada usando regras de quadratura gaussianas para triângulos apresentadas em [12], com ordem de precisão igual $p=13$.

\subsubsection{Teste 1 - Equação de Poisson}

Este teste de validação da ordem foi feito utilizando a função

$$
\phi=\operatorname{sen}(\pi x) \operatorname{sen}(\pi y)
$$


com domínio em um quadrado unitário $\Omega=[0,1] \times[0,1]$. Em (2.24) foi considerado como velocidade de advecção $\omega=(0,0)$ e coeficiente de difusão $\kappa=1$, que resulta na equação de Poisson $\nabla \cdot(\nabla \phi)$. A forma analítica de $\phi$ foi utilizada como condição de fronteira. Ao substituir a função $\phi$ na equação de advecção-difusão, com os dados apresentados, surge um termo fonte dado por

$$
f=-2 \pi^{2} \operatorname{sen}(\pi x) \operatorname{sen}(\pi y)
$$

Dessa forma, a expressão para R fica dada por

$$
\mathrm{R}=\iint_{\Omega}\left[-\nabla^{2} \phi-f\right] d \Omega
$$

A tabela 2.6 apresenta o número de vértices e triângulos para cada uma das malhas utilizadas nessa validação. As ordens obtidas são dadas na tabela 2.7. Os gráficos 2.13, 2.14 e 2.15 refletem o efeito da ordem de reconstrução no cálculo da integral dos fluxos.

\begin{tabular}{ccc}
\hline \hline MALHA & VÉRTICES & TRIÂNGULOS \\
\hline \hline 1 & 145 & 248 \\
2 & 535 & 988 \\
3 & 2074 & 3986 \\
4 & 8078 & 15834 \\
\hline \hline
\end{tabular}

Tabela 2.6: Teste 1 - Parâmetros das malhas no quadrado

\begin{tabular}{cccc}
\hline \hline ORDEM & $\mathrm{L}_{\infty}$ & $\mathrm{L}_{1}$ & $\mathrm{~L}_{2}$ \\
\hline \hline 2 & 1.55 & 2.08 & 2.04 \\
3 & 2.80 & 3.45 & 3.37 \\
4 & 3.48 & 4.12 & 4.05 \\
\hline \hline
\end{tabular}

Tabela 2.7: Teste 1 - volumes de controle internos 


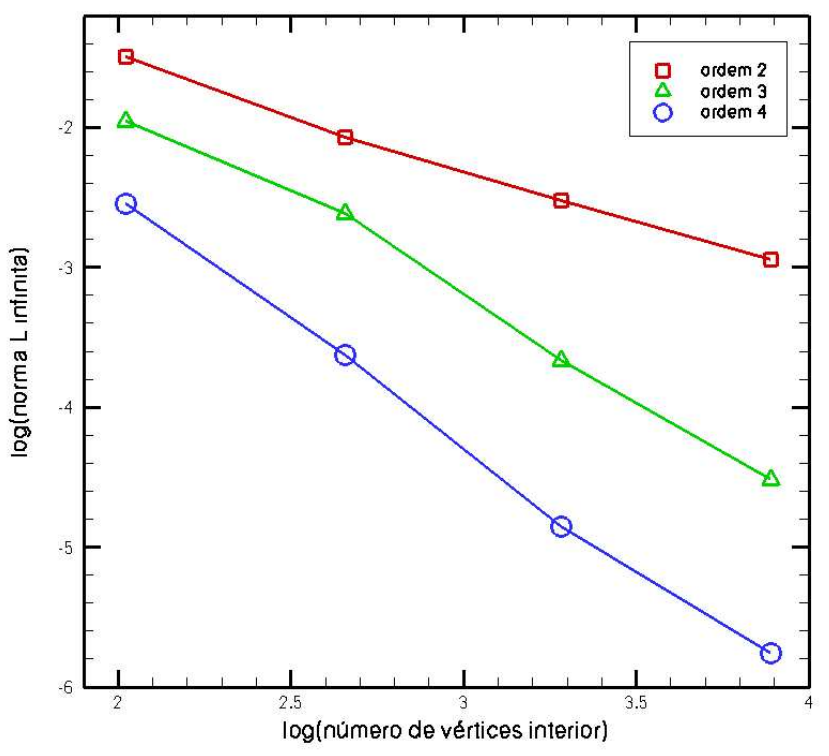

Figura 2.13: Teste 1 - norma $L_{\infty}$

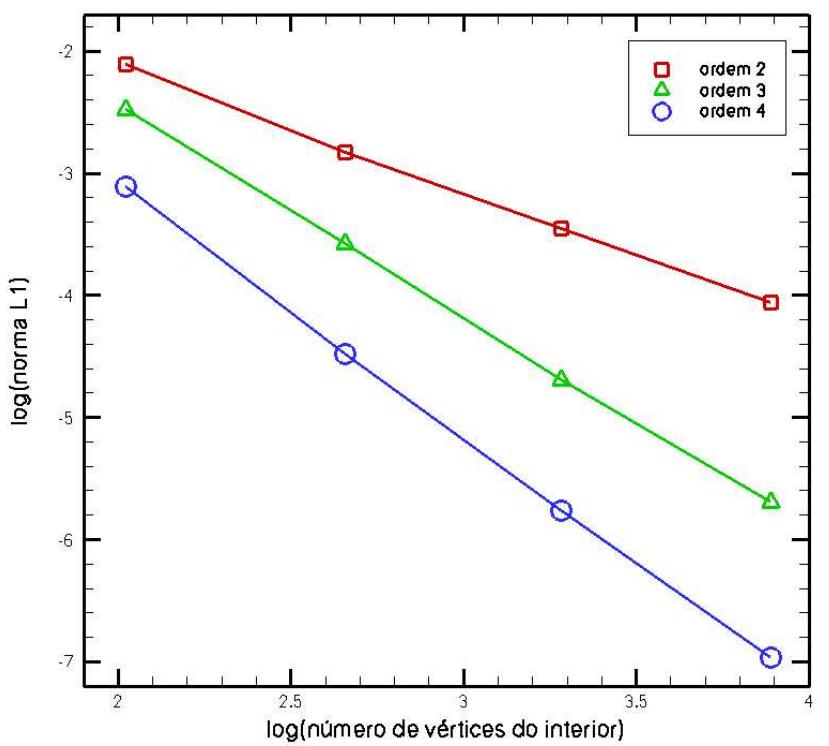

Figura 2.14: Teste 1 - norma $\mathrm{L}_{1}$

A tabela 2.7 mostra que a ordem de precisão da integral dos fluxos reflete a precisão da ordem da reconstrução utilizada. Além disso, os gráficos mostram que o aumento da ordem de reconstrução, como esperado, aumenta a precisão das aproximações. 


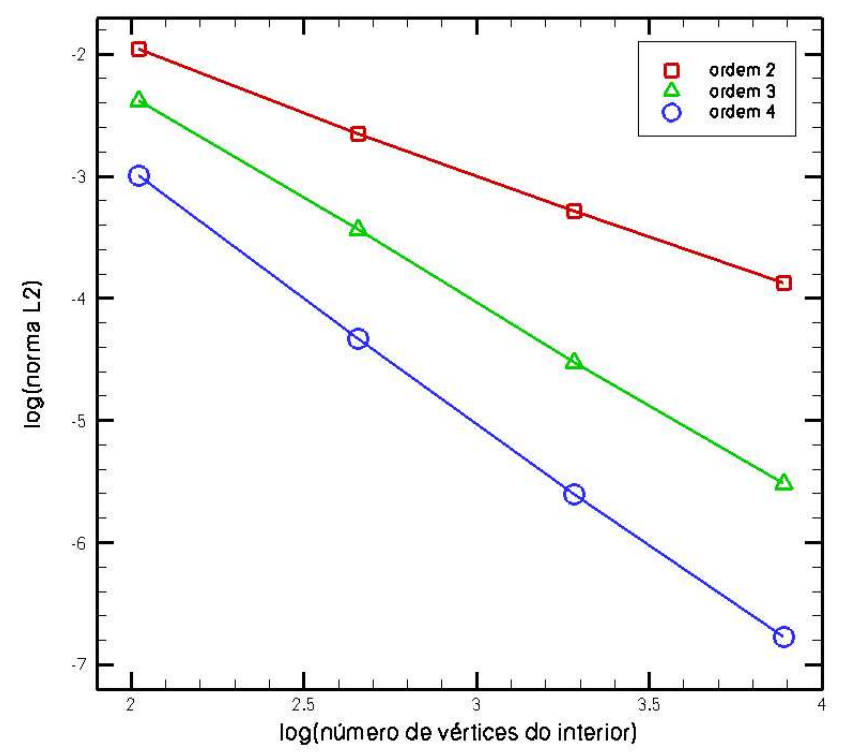

Figura 2.15: Teste 1 - norma $\mathrm{L}_{2}$

\subsubsection{Teste 2 - canal retangular}

O teste de ordem envolvendo fronteiras sob condição de Dirichlet e Neumann é apresentado em Gooch [30]. É um problema advecção-difusão em um canal retangular, o qual é dado por

$$
\nabla \cdot(\vec{\omega} \phi)-\nabla \cdot(\kappa \nabla \phi)=0 \quad(x, y) \in[0,3] \times[0,1]
$$

onde $\vec{\omega}=(u, v)=(1,0)$ e $\kappa=0.01$ (esse coeficiente de difusão equivale a $1 /$ Re em um escoamento viscoso), tendo condições de fronteira definidas por $\phi(x, 0)=\phi(x, 1)=0, \phi(0, y)=0$ e $\nabla \phi(3, y) \cdot n=0$.

A figura 2.16 ilustra o domínio do problema. Em essência, esse problema ilustra uma fenômeno físico no qual uma propriedade é tranportada na fronteira em $x=0$ na direção do vetor $\vec{w}=(1,0)$

A solução exata desse problema é dada por

$$
\begin{gathered}
\phi(x, y)=\operatorname{sen}(\pi y)\left[\frac{r_{2} e^{\left(r_{1} x+3 r_{2}\right)}-r_{1} e^{\left(r_{2} x+3 r_{1}\right)}}{r_{2} e^{3 r_{2}}-r_{1} e^{3 r_{1}}}\right] \\
r_{1,2}=\frac{1}{2} \pm \sqrt{\frac{1}{4}+\pi^{2}}
\end{gathered}
$$




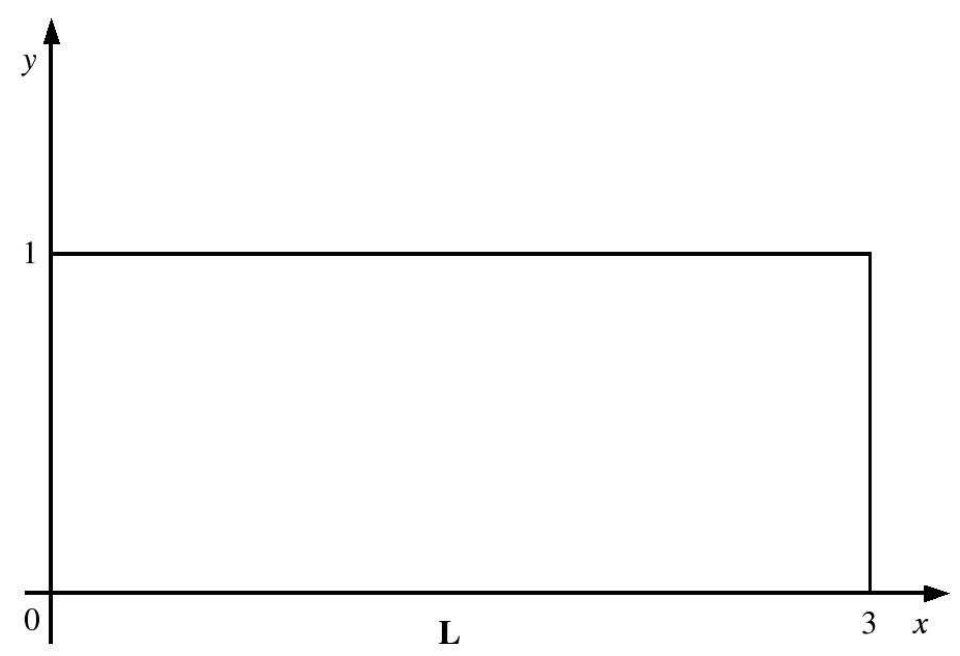

Figura 2.16: Canal retangular

É um teste onde temos 3 fronteiras onde a solução é conhecida e uma, que é a saída em $x=3$, onde apenas a derivada em relação a normal é conhecida, ou seja, 3 fronteiras Dirichlet e 1 Neumann. A reconstrução, na fronteira sob condição de Neumann, para segunda e quarta é feita é feita usando reconstrução de terceira ordem por motivos comentados anteriormente. Assim sendo, como em um teste de ordem o que prevalece é a menor ordem, o esperado nos testes usando reconstrução de segunda ordem e que se tenha como resultado ordem 2, e no de quarta ordem que se tenha ordem 3. A tabela 2.8 mostra os parâmetros das malhas utilizadas e a tabela 2.5 .2 as ordens obtidas. As figuras 2.17, 2.18 e 2.19 mostram o efeito da ordem de reconstrução no cálculo das integrais dos fluxos.

\begin{tabular}{ccc}
\hline \hline MALHA & VÉRTICES & TRIÂNGULOS \\
\hline \hline 1 & 411 & 740 \\
2 & 1560 & 2958 \\
3 & 6067 & 11812 \\
4 & 23829 & 47016 \\
\hline \hline
\end{tabular}

Tabela 2.8: Teste 2 - Parâmetros das malhas no problema do canal retangular

Na tabela 2.9, tem-se resultados que refletem o esperado. Nas reconstruções de segunda e terceira ordem, 


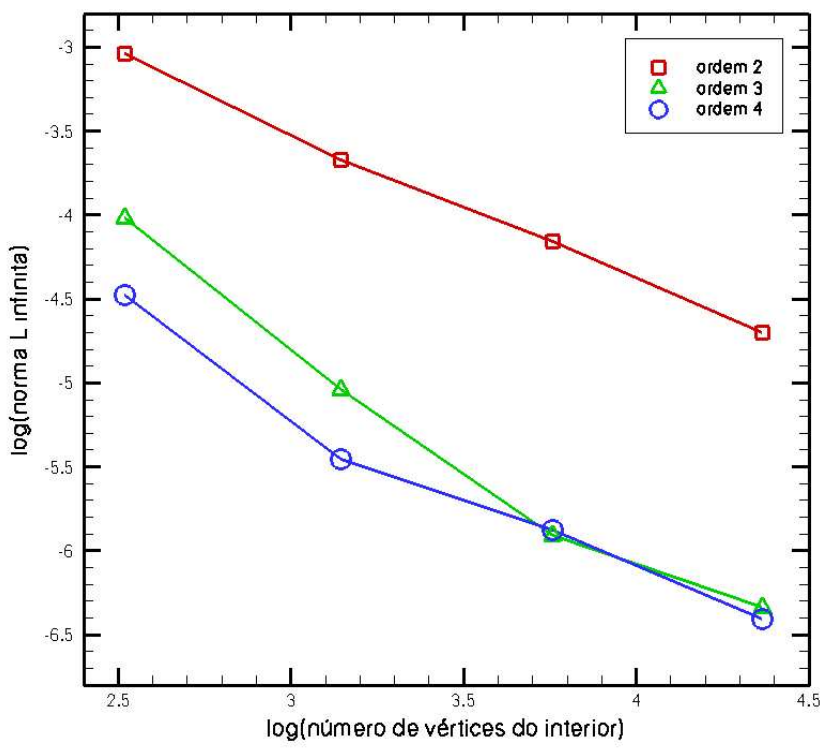

Figura 2.17: Teste 2 - canal retangular - norma $L_{\infty}$

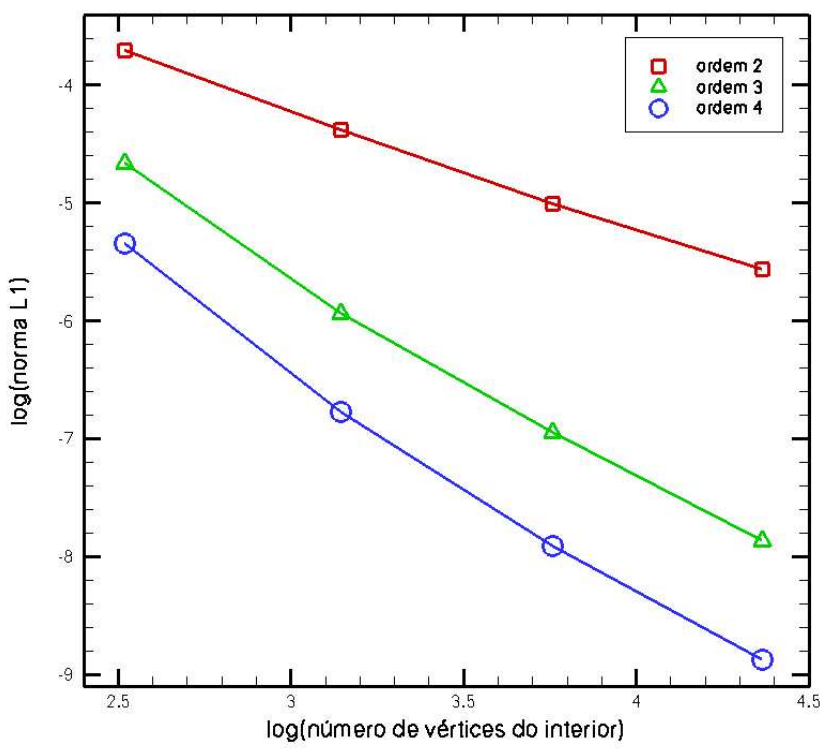

Figura 2.18: Teste 2 - canal retangular - norma $L_{1}$ 


\begin{tabular}{cccc}
\hline \hline ORDEM & $\mathrm{L}_{\infty}$ & $\mathrm{L}_{1}$ & $\mathrm{~L}_{2}$ \\
\hline \hline 2 & 1.78 & 2.01 & 2.00 \\
3 & 2.55 & 3.45 & 3.33 \\
4 & 2.02 & 3.82 & 2.91 \\
\hline \hline
\end{tabular}

Tabela 2.9: Teste 2 - canal retangular - volumes de controle internos

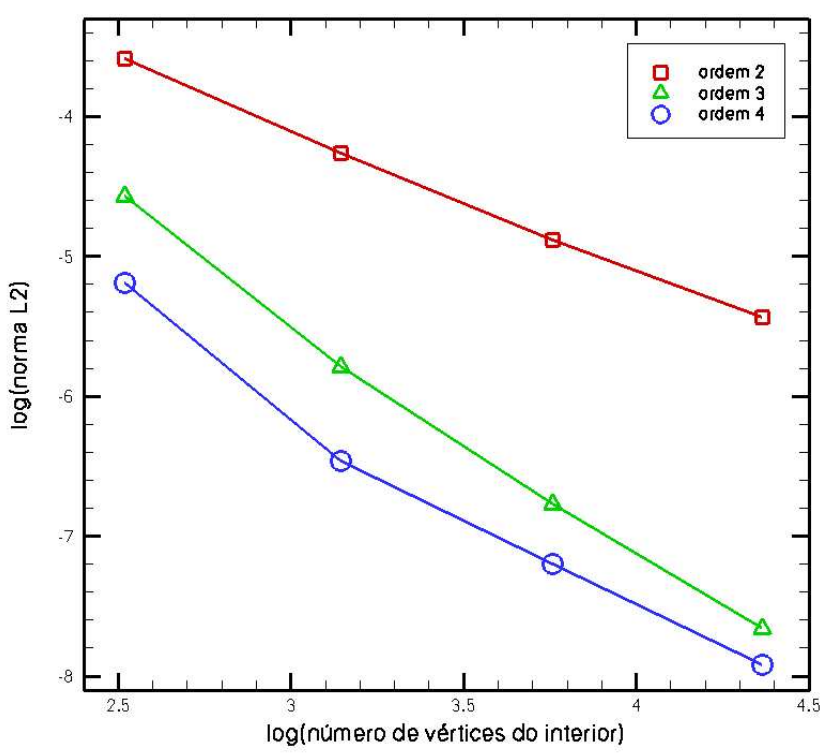

Figura 2.19: Teste 2 - canal retangular - norma $L_{2}$

as ordens se mantiveram. Na reconstrução de quarta ordem, isso já não ocorreu. Embora nos pontos do interior e das fronteiras, onde os valores da solução são conhecidos, tenhamos reconstrução de quarta ordem, na fronteira de Neuman temos reconstrução de terceira ordem. Isso se refletiu nos resultados que constam na tabela 2.5.2. Perceba que para $L_{2}$ na reconstrução de quarta ordem o que se obteve foi ordem 3.

O método de reconstrução de alta ordem, no que tange a sua implementação, tem a vantagem de que a estrutura do código se preserva. Note que nessa técnica, a dimensão das colunas do sistema linear é definida de acordo com a ordem da reconstrução utilizada, assim como o número de linhas, o qual deve respeitar a quantidade mínima, embora possa excedê-la, de vértices vizinhos necessários para cada ordem de reconstrução. Uma vez definida a ordem de reconstrução, e de posse da estrutura de dados da malha, perceba que a construção das matrizes dos coeficientes dos sistemas dependem apenas de informações das variáveis 
espaciais. A obtenção dos elementos dessas matrizes, para cada volume de controle, consiste essencialmente em integrar polinômios. Uma vez formadas, tais matrizes são sempre as mesmas, variando apenas o vetor dos termos independentes, durante a aplicação dessa técnica de reconstrução na resolução numérica de uma EDP via MVF.

A análise de ordem mostrou a efetividade do método. Em problemas onde o refinamento adaptativo da malha é uma necessidade para estudar com mais efetividade um determinado fenômeno físico, o método de reconstrução de alta ordem pode ser aplicado. Nesse caso, pode se fazer uso de uma malha mais grossa em regiões onde a propriedade associada ao fenômeno não tem grandes oscilações de gradientes. Se utilizarmos então reconstrução de quarta ordem, teremos boas aproximações para solução tanto onde a malha é mais grossa quanto onde é mais fina, sendo que na mais fina a ordem elevada irá capturar melhor o comportamento da propriedade física. No que tange as limitações de memória dos computadores, para resolver problemas que exigem uma quantidade enorme de vértices na malha, a técnica de reconstrução de alta ordem é um método conveniente para ser aplicado. Essa situação pode ser resolvida utilizando-se uma malha com um número menor de vértices e uma reconstrução de ordem mais elevada. Desse modo, o MVF que emprega essa técnica de reconstrução, para resolver uma determinada equação diferencial, irá gerar boas aproximações sem que haja necessidade de refinar muito a malha.

\subsection{O efeito da difusão numérica}

A difusão numérica na resolução numérica de uma EDP é proveniente dos erros de discretização provocados pelo método númerico utilizado. Para ilustrar o efeito da difusão numérica, considere a equação de advecção

$$
\nabla \cdot(\vec{\omega} \phi)=0 \quad(x, y) \in[0,1] \times[0,1]
$$

definida em um quadrado unitário, sendo a velocidade de advecção definida por $\vec{\omega}=(1,0)$, e suas condições de fronteira dadas por

$$
\begin{aligned}
& \phi(0, y)=\left\{\begin{array}{ll}
1, & 0 \leq y \leq 0.4 \\
5 y-1, & 0.4<y<0.6 \\
2, & 0.6 \leq y \leq 1.0
\end{array} \quad \phi(x, 0)=1 \quad 0<x \leq 1\right. \\
& \phi(x, 1)=2 \quad 0<x \leq 1 \quad \frac{\partial \phi(1, y)}{\partial n}=0 \quad 0<y<1
\end{aligned}
$$


Nessa ilustração, a equação de advecção (2.28) é resolvida em três malhas, cujas informações constam na tabela 2.10 .

\begin{tabular}{ccc}
\hline \hline MALHA & VÉRTICES & TRIÂNGULOS \\
\hline \hline 1 & 145 & 248 \\
2 & 314 & 566 \\
3 & 535 & 988 \\
\hline \hline
\end{tabular}

Tabela 2.10: Tabela com número de vértices e triângulos das malhas de triângulos

Com relação ao critério de parada, considerou-se a norma 2 do resíduo com tolerância $10^{-6}$. A tabela 2.11 apresenta o número de iterações e o tempo gasto para cada uma das três malhas.

\begin{tabular}{ccc}
\hline \hline MALHA & ITERAÇÕES & TEMPO $(\mathrm{seg})$ \\
\hline \hline 1 & 538 & 6 \\
2 & 664 & 17 \\
3 & 771 & 33 \\
\hline \hline
\end{tabular}

Tabela 2.11: Tabela com número de vértices e triângulos das malhas de triângulos

Por ser uma equação de puramente de transporte, a solução em $x=0$ tem que ser apenas transportada na direção do vetor $\vec{\omega}=(1,0)$. Utilizando reconstrução de terceira ordem, foram feitos dois testes. Uma com a malha 1 e outra com a malha 3. Perceba que a malha 1 (figura 2.20), que é uma malha grossa, mostra claramente o efeito da difusão numérica. Abre-se um leque característico de fenômenos difusivos. Já na malha 3 (figura 2.21), o efeito da difusão numérica é reduzido. Essa redução foi provocada pelo refinamento da malha.

No problema de estimar o coeficiente de difusão $\kappa$, da equação de advecção-difusão, a difusão numérica tende a dificultar a identificação dos parâmetros $\beta_{k}$ que definem $\kappa$. Toda malha, por mais fina que seja, apresenta alguma difusão numérica. O refinamento, como comentado, ameniza os efeitos da difusão numérica. Assim sendo, os resultados, que serão apresentado no capítulo 4, irão mostrar o que ocorre quando se utiliza o método adjunto, bem como o método de reconstrução de alta ordem, no processo de identificação de parâmetros. Os resultados obtidos calculando os gradientes via método adjunto e via diferenças finitas são comparados, mostrando os efeitos da difusão numérica sobre o problema de identificação de parâmetros.

O próximo capítulo apresenta a técnica do operador adjunto, detalhando o desenvolvimento da equação adjunta bem o processo de cálculo dos gradientes. 


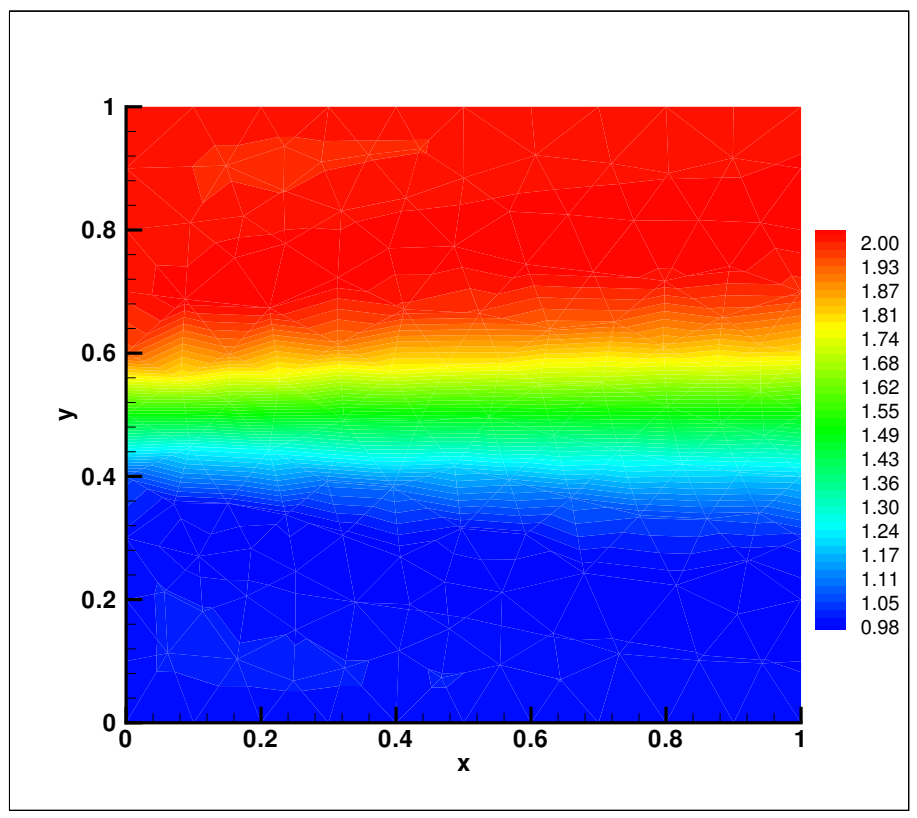

Figura 2.20: Efeito da difusão numérica na malha 1

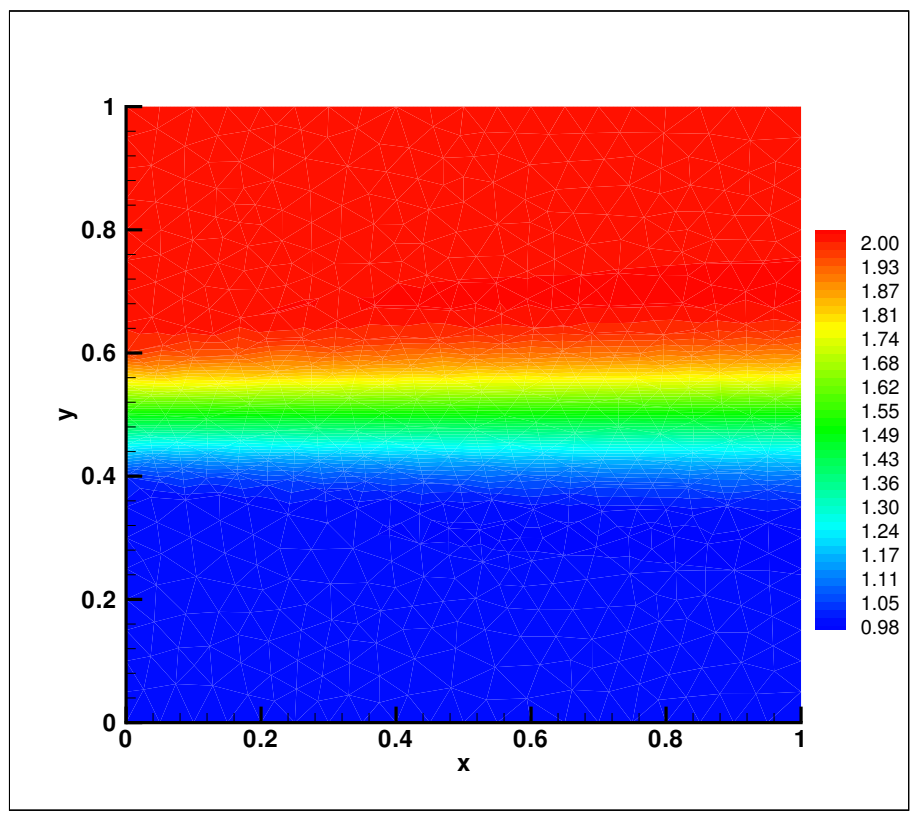

Figura 2.21: Efeito da difusão numérica na malha 3 


\section{Método do operador adjunto para identificação de parâmetros}

\subsection{Definição do problema geral}

O problema de identificação de parâmetros tratado nesse trabalho consiste em estimar os $\mathrm{N}$ coeficientes $\beta_{\mathrm{k}}$ que definem o coeficiente de difusão na forma

$$
\kappa(x, y)=C_{0}+\sum_{\mathrm{k}=1}^{\mathrm{N}} \beta_{\mathrm{k}} C_{\mathrm{k}}(x, y)
$$

sendo $C_{0}$ uma constante e $C_{\mathrm{k}}(x, y)$ um conjunto de $\mathrm{N}$ funções previamente escolhidas, de tal modo que o campo medido do escalar $\phi_{\mathrm{R}}$ seja recuperado. Para tal, deseja-se minimizar o funcional

$$
\mathcal{F}[\kappa]=\frac{1}{2} \iint_{\Omega}\left(\phi-\phi_{\mathrm{R}}\right)^{2} d \Omega
$$

restrito a equação de advecção-difusão

$$
\nabla \cdot(\vec{\omega} \phi)-\nabla \cdot(\kappa \nabla \phi)=0 \quad(x, y) \in \Omega \subset \mathbb{R}^{2}
$$

sendo $\vec{\omega}=(u, v) \in \mathbb{R}^{2}$ a velocidade de advecção com componentes $u$ e $v$, conhecidas em cada ponto do domínio, e $\kappa$ o coeficiente de difusão variável em $\Omega$.

Dependendo da geometria do problema, consideram-se condições de fronteira do tipo Dirichlet e Neumann em porções distintas do contorno 


$$
\begin{cases}\phi=f, & (x, y) \in \partial \Omega_{1} \\ \nabla \phi \cdot n=g, & (x, y) \in \partial \Omega_{2}\end{cases}
$$

onde $\partial \Omega=\partial \Omega_{1} \cup \partial \Omega_{2}$.

A equação de restrição (3.3) com suas condições de fronteira é conhecido como problema primal.

O integrando no funcional caracteriza um problema inverso e o problema de identificação de parâmetros, o qual consiste em determinar os referidos coeficientes de tal forma que a equação de restrição seja satisfeita e que o funcional seja minimizado, caracteriza um problema de otimização. A referência $\phi_{\mathrm{R}}$ está ligada a EDP de restrição e é o efeito de algum coeficiente de difusão $k$. O que pretende-se aqui é estimar a causa desse efeito na forma da função (3.51) estimando os valores dos coeficientes $\beta_{\mathrm{k}}$.

Os desenvolvimentos a seguir apresentam os detalhamentos teóricos envolvidos na obtenção da equação adjunta, suas condições de fronteira e o processo de cálculo dos gradientes. Os cálculos seguem as técnicas dos trabalhos de Orlande [7, 49] e Giles [15].

\subsection{Cálculo das sensibilidades}

A equação de sensibilidade tem por finalidade fornecer a função de sensibilidade $\delta_{\kappa} \phi$, a qual é definida como sendo a derivada direcional, da função incógnita $\phi$ da restrição do problema direto, na direção de perturbação do parâmetro que está sendo estimado $[1,40]$. Isso significa que $\delta_{\kappa} \phi$ é a variação em $\phi$ provocada pela variação em $\kappa$. Para obter a equação de sensibilidade utiliza-se algumas idéias bem simples.

Seja $\mathcal{L}$ o operador definido por

$$
\mathcal{L} \phi=\nabla \cdot(\vec{\omega} \phi)-\nabla \cdot(\kappa \nabla \phi)
$$

Utilizando esse operador, temos a partir de (3.3) que o resíduo tem a forma

$$
\mathcal{R}=\mathcal{L} \phi=0
$$

Uma perturbação $\varepsilon \delta \kappa$ em $\kappa$ irá provocar uma variação $\varepsilon \delta_{\kappa} \phi$ em $\phi$. Com isso, definimos o operador 
perturbado $\mathcal{L}_{\kappa}$ por

$$
\mathcal{L}_{\kappa} \phi=\nabla \cdot\left[\vec{\omega}\left(\phi+\varepsilon \delta_{\kappa} \phi\right)\right]-\nabla \cdot\left[(\kappa+\varepsilon \delta \kappa) \nabla\left(\phi+\varepsilon \delta_{\kappa} \phi\right)\right]
$$

Considerando essas perturbações provocadas em cada membro da equação (3.3), segue que

$$
\mathcal{L}_{\kappa} \phi=0
$$

Subtraindo (3.8) de (3.6) e passando ao limite com $\varepsilon$ tendendo a zero,

$$
\delta_{\kappa} \mathcal{R}=\lim _{\varepsilon \rightarrow 0} \frac{\mathcal{L}_{\kappa} \phi-\mathcal{L} \phi}{\varepsilon}=0
$$

a qual é chamada derivada de Gâteaux.

Abrindo $\mathcal{L}_{\kappa} \phi$, temos

$$
\mathcal{L}_{\kappa} \phi=\nabla \cdot\left[\vec{\omega}\left(\phi+\varepsilon \delta_{\kappa} \phi\right)\right]-\nabla \cdot\left[\kappa \nabla\left(\phi+\varepsilon \delta_{\kappa} \phi\right)\right]
$$

$$
\mathcal{L}_{\kappa} \phi=\frac{\partial u\left(\phi+\varepsilon \delta_{\kappa} \phi\right)}{\partial x}+\frac{\partial v\left(\phi+\varepsilon \delta_{\kappa} \phi\right)}{\partial y}-\frac{\partial}{\partial x}\left[(\kappa+\varepsilon \delta \kappa) \frac{\partial\left(\phi+\varepsilon \delta_{\kappa} \phi\right)}{\partial x}\right]-\frac{\partial}{\partial y}\left[(\kappa+\varepsilon \delta \kappa) \frac{\partial\left(\phi+\varepsilon \delta_{\kappa} \phi\right)}{\partial y}\right]
$$

$$
\mathcal{L}_{\kappa} \phi=\frac{\partial u \phi}{\partial x}+\frac{\partial v \phi}{\partial y}+\frac{\partial u \varepsilon \delta_{\kappa} \phi}{\partial x}+\frac{\partial v \varepsilon \delta_{\kappa} \phi}{\partial y}-\frac{\partial}{\partial x}\left[(\kappa+\varepsilon \delta \kappa)\left(\frac{\partial \phi}{\partial x}+\frac{\partial \varepsilon \delta_{\kappa} \phi}{\partial x}\right)\right]-\frac{\partial}{\partial y}\left[(\kappa+\varepsilon \delta \kappa)\left(\frac{\partial \phi}{\partial y}+\frac{\partial \varepsilon \delta_{\kappa} \phi}{\partial y}\right)\right]
$$




$$
\begin{gathered}
\mathcal{L}_{\kappa} \phi=\frac{\partial u \phi}{\partial x}+\frac{\partial v \phi}{\partial y}+\varepsilon \frac{\partial u \delta_{\kappa} \phi}{\partial x}+\varepsilon \frac{\partial v \delta_{\kappa} \phi}{\partial y}-\frac{\partial}{\partial x}\left(\kappa \frac{\partial \phi}{\partial x}+\varepsilon \kappa \frac{\partial \delta_{\kappa} \phi}{\partial x}+\varepsilon \delta \kappa \frac{\partial \phi}{\partial x}+\varepsilon^{2} \delta \kappa \frac{\partial \delta_{\kappa} \phi}{\partial x}\right)- \\
\frac{\partial}{\partial y}\left(\kappa \frac{\partial \phi}{\partial y}+\varepsilon \kappa \frac{\partial \delta_{\kappa} \phi}{\partial y}+\varepsilon \delta \kappa \frac{\partial \phi}{\partial y}+\varepsilon^{2} \delta \kappa \frac{\partial \delta_{\kappa} \phi}{\partial y}\right) \\
\mathcal{L}_{\kappa} \phi=\frac{\partial u \phi}{\partial x}+\frac{\partial v \phi}{\partial y}+\varepsilon \frac{\partial u \delta_{\kappa} \phi}{\partial x}+\varepsilon \frac{\partial v \delta_{\kappa} \phi}{\partial y}-\frac{\partial}{\partial x}\left(\kappa \frac{\partial \phi}{\partial x}\right)-\varepsilon \frac{\partial}{\partial x}\left(\kappa \frac{\partial \delta_{\kappa} \phi}{\partial x}\right)-\varepsilon \frac{\partial}{\partial x}\left(\delta \kappa \frac{\partial \phi}{\partial x}\right)-\varepsilon^{2} \frac{\partial}{\partial x}\left(\delta \kappa \frac{\partial \delta_{\kappa} \phi}{\partial x}\right)- \\
\frac{\partial}{\partial y}\left(\kappa \frac{\partial \phi}{\partial y}\right)-\varepsilon \frac{\partial}{\partial y}\left(\kappa \frac{\partial \delta_{\kappa} \phi}{\partial y}\right)-\varepsilon \frac{\partial}{\partial y}\left(\delta \kappa \frac{\partial \phi}{\partial y}\right)-\varepsilon^{2} \frac{\partial}{\partial y}\left(\delta \kappa \frac{\partial \delta_{\kappa} \phi}{\partial y}\right)
\end{gathered}
$$

Substituindo $\mathcal{L}_{\kappa} \phi$ e $\mathcal{L} \phi$ em (3.9) e calculando o limite chegaremos a

$$
\nabla \cdot\left(\vec{\omega} \delta_{\kappa} \phi\right)-\nabla \cdot\left[\kappa \nabla \delta_{\kappa} \phi+\delta \kappa \nabla \phi\right]=0
$$

a qual define a chamada equação de sensibilidade, com condições de fronteira dadas por

$$
\begin{cases}\delta_{\kappa} \phi=0, & (x, y) \in \partial \Omega_{1} \\ \nabla \delta_{\kappa} \phi \cdot n=0, & (x, y) \in \partial \Omega_{2}\end{cases}
$$

uma vez que $f$ e $g$ não dependem de $\kappa$. As condições de fronteira (3.12) são complementares as condições de fronteira do problema primal (3.4).

Considerando esse mesmo princípio de cálculo, podemos calcular também a variação em $\mathcal{F}$ provocada por variações em $\kappa$. Assim sendo, se $\mathcal{F}$ é o funcional

$$
\mathcal{F}[\kappa]=\frac{1}{2} \iint_{\Omega}\left(\phi-\phi_{\mathrm{R}}\right)^{2} d \Omega
$$

e $\mathcal{F}_{\kappa}$ o funcional perturbado pela perturbação em $\kappa$, que provocou por sua vez uma perturbação em $\phi$ dada por $\varepsilon \delta_{\kappa} \phi$, 


$$
\mathcal{F}_{\kappa}[\kappa]=\frac{1}{2} \iint_{\Omega}\left[\left(\phi+\varepsilon \delta_{\kappa} \phi\right)-\phi_{\mathrm{R}}\right]^{2} d \Omega
$$

Calculando então, segue

$$
\begin{aligned}
& \delta_{\kappa} \mathcal{F}=\lim _{\varepsilon \rightarrow 0} \frac{\mathcal{F}_{\kappa}[\phi(\kappa)]-\mathcal{F}[\phi(\kappa)]}{\varepsilon} \\
& \frac{1}{2} \iint\left[\left(\phi+\varepsilon \delta_{\kappa} \phi\right)-\phi_{\mathrm{R}}\right]^{2} d \Omega-\frac{1}{2} \iint\left(\phi-\phi_{\mathrm{R}}\right)^{2} d \Omega \\
& =\lim _{\varepsilon \rightarrow 0} \frac{\int_{\Omega}}{\varepsilon} \\
& =\lim _{\varepsilon \rightarrow 0} \frac{1}{2 \varepsilon} \iint_{\Omega}\left[\left(\phi+\varepsilon \delta_{\kappa} \phi\right)-\phi_{\mathrm{R}}\right]^{2}-\left(\phi-\phi_{\mathrm{R}}\right)^{2} d \Omega \\
& =\lim _{\varepsilon \rightarrow 0} \frac{1}{2 \varepsilon} \iint_{\Omega}\left[\left(\phi+\varepsilon \delta_{\kappa} \phi\right)^{2}-2\left(\phi+\varepsilon \delta_{\kappa} \phi\right) \phi_{\mathrm{R}}+\phi_{\mathrm{R}}^{2}-\left(\phi-\phi_{\mathrm{R}}\right)^{2}\right] d \Omega \\
& =\lim _{\varepsilon \rightarrow 0} \frac{1}{2 \varepsilon} \iint_{\Omega}\left(\phi^{2}+2 \phi \varepsilon \delta_{\kappa} \phi+\varepsilon^{2} \delta_{\kappa} \phi^{2}-2 \phi \phi_{\mathrm{R}}-2 \varepsilon \delta_{\kappa} \phi \phi_{\mathrm{R}}+\phi_{\mathrm{R}}^{2}-\phi^{2}+2 \phi \phi_{\mathrm{R}}-\phi_{\mathrm{R}}^{2}\right) d \Omega \\
& =\lim _{\varepsilon \rightarrow 0} \frac{1}{2 \varepsilon} \iint_{\Omega}\left(2 \phi \varepsilon \delta_{\kappa} \phi+\varepsilon^{2} \delta_{\kappa} \phi^{2}-2 \varepsilon \delta_{\kappa} \phi \phi_{\mathrm{R}}\right) d \Omega \\
& =\lim _{\varepsilon \rightarrow 0} \iint_{\Omega}\left(\phi \delta_{\kappa} \phi+\frac{\varepsilon \delta_{\kappa} \phi^{2}}{2}-\delta_{\kappa} \phi \phi_{\mathrm{R}}\right) d \Omega \\
& =\iint_{\Omega}\left(\phi-\phi_{\mathrm{R}}\right) \delta_{\kappa} \phi d \Omega . \\
& \delta_{\kappa} \mathcal{F}=\iint_{\Omega}\left(\phi-\phi_{\mathrm{R}}\right) \delta_{\kappa} \phi d \Omega
\end{aligned}
$$

Esses resultados serão empregados na obtenção da equação adjunta bem como das condições de fronteira. 


\subsection{Obtenção da equação adjunta}

Considere o variação do funcional $\mathcal{F}$ em relação a $\kappa$ aumentado

$$
\delta_{\kappa} \mathcal{F}=\delta_{\kappa} \mathcal{F}+\left\langle\psi, \delta_{\kappa} \mathcal{R}\right\rangle
$$

onde

$$
\left\langle\psi, \delta_{\kappa} \mathcal{R}\right\rangle=\iint_{\Omega} \psi \delta_{\kappa} \mathcal{R} d \Omega
$$

sendo $\psi$, uma função nas variáveis $x$ e $y$ definidas no mesmo domínio da função $\phi$, solução do problema primal. A função $\psi$ é a função incógnita na equação adjunta que será obtida nos desenvolvimentos. Perceba que o termo

$$
\left\langle\psi, \delta_{\kappa} \mathcal{R}\right\rangle=0
$$

é nulo, pois $\delta_{\kappa} \mathcal{R}=0$. Logo, ao somar esse produto interno o gradiente não está sendo alterado.

Continuando, da equação (3.16), temos

$$
\delta_{\kappa} \mathcal{F}=\iint_{\Omega}\left(\phi-\phi_{\mathrm{R}}\right) \delta_{\kappa} \phi d \Omega+\iint_{\Omega} \psi\left[\nabla \cdot\left(\vec{\omega} \delta_{\kappa} \phi\right)-\nabla \cdot\left(\kappa \nabla \delta_{\kappa} \phi+\delta \kappa \nabla \phi\right)\right] d \Omega
$$

As contas agora ficarão concentradas sobre

$$
\begin{gathered}
\mathcal{I}_{\mathrm{AD}}=\iint_{\Omega} \psi\left[\nabla \cdot\left(\vec{\omega} \delta_{\kappa} \phi\right)-\nabla \cdot\left(\kappa \nabla \delta_{\kappa} \phi+\delta \kappa \nabla \phi\right)\right] d \Omega \\
\mathcal{I}_{\mathrm{AD}}=\underbrace{\iint_{\Omega} \psi\left[\nabla \cdot\left(\vec{\omega} \delta_{\kappa} \phi\right)\right] d \Omega}_{\mathcal{I}_{\mathrm{A}}}-\underbrace{\iint_{\Omega}}_{\mathcal{I}_{\mathrm{D}} \iint_{\Omega} \psi\left[\nabla \cdot\left(\kappa \nabla \delta_{\kappa} \phi+\delta \kappa \nabla \phi\right)\right] d \Omega}
\end{gathered}
$$


e a técnica de integração por partes será constantemente empregada.

\subsubsection{Integração da parte advectiva}

De (3.18) temos a parte advectiva dada por

$$
\begin{aligned}
& \mathcal{I}_{\mathrm{A}}=\iint_{\Omega} \psi\left[\nabla \cdot\left(\vec{\omega} \delta_{\kappa} \phi\right)\right] d \Omega \\
&=\iint_{\Omega} \psi\left[\frac{\partial\left(u \delta_{\kappa} \phi\right)}{\partial x}+\frac{\partial\left(v \delta_{\kappa} \phi\right)}{\partial y}\right] d \Omega \\
&=\underbrace{\iint_{\Omega} \psi \frac{\partial\left(u \delta_{\kappa} \phi\right)}{\partial x} d \Omega}_{\mathcal{I}_{\mathrm{A}_{1}}}+\underbrace{\iint_{\Omega} \psi \frac{\partial\left(v \delta_{\kappa} \phi\right)}{\partial y} d \Omega}_{\mathcal{I}_{\mathrm{A}_{2}}} \\
& \mathcal{I}_{\mathrm{A}}=\mathcal{I}_{\mathrm{A}_{1}}+\mathcal{I}_{\mathrm{A}_{2}}
\end{aligned}
$$

A integral da parte advectiva fica dividida em duas partes, $\mathcal{I}_{\mathrm{A}_{1}}$ e $\mathcal{I}_{\mathrm{A}_{2}}$, as quais serão agora integradas utilizando a técnica de integração por partes.

Cálculo de $\mathcal{I}_{\mathrm{A}_{1}}$ :

$$
\mathcal{I}_{\mathrm{A}_{1}}=\iint_{\Omega} \psi \frac{\partial\left(u \delta_{\kappa} \phi\right)}{\partial x} d \Omega=\int_{c}^{d} \underbrace{\left[\int_{g_{1}(y)}^{g_{2}(y)} \psi \frac{\partial\left(u \delta_{\kappa} \phi\right)}{\partial x} d x\right]}_{\mathcal{I}_{\mathrm{A}_{1 x}}} d y
$$

Integrando $\mathcal{I}_{\mathrm{A}_{1 x}}$ por partes, segue

$$
\mathcal{I}_{\mathrm{A}_{1 x}}=\int_{g_{1}(y)}^{g_{2}(y)} \psi \frac{\partial\left(u \delta_{\kappa} \phi\right)}{\partial x} d x=\left[\psi u \delta_{\kappa} \phi\right]_{g_{1}(y)}^{g_{2}(y)}-\int_{g_{1}(y)}^{g_{2}(y)} u \delta_{\kappa} \phi \frac{\partial \psi}{\partial x} d x
$$




$$
\left\{\begin{array}{l}
p=\psi \Rightarrow d p=\frac{\partial \psi}{\partial x} d x \\
d q=\frac{\partial\left(u \delta_{\kappa} \phi\right)}{\partial x} d x \Rightarrow q=u \delta_{\kappa} \phi
\end{array}\right.
$$

Substituindo (3.21) em (3.20),

$$
\mathcal{I}_{\mathrm{A}_{1}}=\int_{c}^{d}\left\{\left[\psi u \delta_{\kappa} \phi\right]_{g_{1}(y)}^{g_{2}(y)}-\int_{g_{1}(y)}^{g_{2}(y)} u \delta_{\kappa} \phi \frac{\partial \psi}{\partial x} d x\right\} d y=\int_{c}^{d}\left[\psi u \delta_{\kappa} \phi\right]_{g_{1}(y)}^{g_{2}(y)} d y-\int_{c}^{d} \int_{g_{1}(y)}^{g_{2}(y)} u \delta_{\kappa} \phi \frac{\partial \psi}{\partial x} d x d y
$$

e obtém-se $\mathcal{I}_{\mathrm{A}_{1}}$, o qual é dado por

$$
\mathcal{I}_{\mathrm{A}_{1}}=\oint_{\partial \Omega} \psi u \delta_{\kappa} \phi n_{x} d s-\iint_{\Omega} u \delta_{\kappa} \phi \frac{\partial \psi}{\partial x} d \Omega
$$

Cálculo de $\mathcal{I}_{\mathrm{A}_{2}}$ :

$$
\mathcal{I}_{\mathrm{A}_{2}}=\iint_{\Omega} \psi \frac{\partial\left(v \delta_{\kappa} \phi\right)}{\partial y} d \Omega=\int_{a}^{b} \underbrace{\left[\int_{f_{1}(x)}^{f_{2}(x)} \psi \frac{\partial\left(v \delta_{\kappa} \phi\right)}{\partial y} d y\right]}_{\mathcal{I}_{\mathrm{A}_{2 y}}} d x
$$

Integrando $\mathcal{I}_{\mathrm{A}_{2 y}}$ por partes, segue

$$
\begin{gathered}
\mathcal{I}_{\mathrm{A}_{2 y}}=\int_{f_{1}(x)}^{f_{2}(x)} \psi \frac{\partial\left(v \delta_{\kappa} \phi\right)}{\partial y} d y=\left[\psi v \delta_{\kappa} \phi\right]_{f_{1}(x)}^{f_{2}(x)}-\int_{f_{1}(x)}^{f_{2}(x)} v \delta_{\kappa} \phi \frac{\partial \psi}{\partial y} d y \\
\left\{\begin{array}{l}
p=\psi \Rightarrow d p=\frac{\partial \psi}{\partial y} d y \\
d q=\frac{\partial\left(v \delta_{\kappa} \phi\right)}{\partial y} d y \Rightarrow q=v \delta_{\kappa} \phi
\end{array}\right.
\end{gathered}
$$


Substituindo (3.24) (3.23),

$$
\mathcal{I}_{\mathrm{A}_{2}}=\int_{a}^{b}\left\{\left[\psi v \delta_{\kappa} \phi\right]_{f_{1}(x)}^{f_{2}(x)}-\int_{f_{1}(x)}^{f_{2}(x)} v \delta_{\kappa} \phi \frac{\partial \psi}{\partial y} d y\right\} d x=\int_{a}^{b}\left[\psi v \delta_{\kappa} \phi\right]_{f_{1}(x)}^{f_{2}(x)} d x-\int_{a}^{b} \int_{f_{1}(x)}^{f_{2}(x)} v \delta_{\kappa} \phi \frac{\partial \psi}{\partial y} d x d y
$$

e obtém-se $\mathcal{I}_{\mathrm{A}_{2}}$, o qual é dado por

$$
\mathcal{I}_{\mathrm{A}_{2}}=\oint_{\partial \Omega} \psi v \delta_{\kappa} \phi n_{y} d s-\iint_{\Omega} v \delta_{\kappa} \phi \frac{\partial \psi}{\partial y} d \Omega
$$

Substituindo (3.22) e (3.25) em (3.19),

$$
\begin{aligned}
\mathcal{I}_{\mathrm{A}} & =\mathcal{I}_{\mathrm{A}_{1}}+\mathcal{I}_{\mathrm{A}_{2}} \\
& =\oint_{\partial \Omega} \psi u \delta_{\kappa} \phi n_{x} d s-\iint_{\Omega} u \delta_{\kappa} \phi \frac{\partial \psi}{\partial x} d \Omega+\oint_{\partial \Omega} \psi v \delta_{\kappa} \phi n_{y} d s-\iint_{\Omega} v \delta_{\kappa} \phi \frac{\partial \psi}{\partial y} d \Omega \\
& =\oint_{\partial \Omega} \psi \delta_{\kappa} \phi\left(u n_{x}+v n_{y}\right) d s-\iint_{\Omega} \delta_{\kappa} \phi\left(u \frac{\partial \psi}{\partial x}+v \frac{\partial \psi}{\partial y}\right) d \Omega
\end{aligned}
$$

Finalizando, a integral da parte advectiva então é dada por

$$
\mathcal{I}_{\mathrm{A}}=\oint_{\partial \Omega} \psi \delta_{\kappa} \phi \vec{\omega} \cdot n d s-\iint_{\Omega} \delta_{\kappa} \phi \vec{\omega} \cdot \nabla \psi d \Omega
$$

Continuando, segue a integração da parte difusiva.

\subsubsection{Integração da parte difusiva}

De (3.18), temos a parte difusiva dada por

$$
\mathcal{I}_{\mathrm{D}}=\iint_{\Omega} \psi\left[\nabla \cdot\left(\kappa \nabla \delta_{\kappa} \phi+\delta \kappa \nabla \phi\right)\right] d \Omega=\underbrace{\iint_{\Omega} \psi\left[\nabla \cdot\left(\kappa \nabla \delta_{\kappa} \phi\right)\right] d \Omega}_{\mathcal{I}_{\mathrm{D}_{1}}}+\underbrace{\int_{\Omega} \psi[\nabla \cdot(\delta \kappa \nabla \phi)] d \Omega}_{\mathcal{I}_{\mathrm{D}_{2}}}
$$




$$
\mathcal{I}_{\mathrm{D}}=\mathcal{I}_{\mathrm{D}_{1}}+\mathcal{I}_{\mathrm{D}_{2}}
$$

A integral da parte difusiva fica dividida em duas partes, $\mathcal{I}_{\mathrm{D}_{1}}$ e $\mathcal{I}_{\mathrm{D}_{2}}$, as quais serão agora integradas utilizando, assim como foi feito na parte advectiva, a técnica de integração por partes.

Cálculo de $\mathcal{I}_{\mathrm{D}_{1}}$ :

$$
\begin{aligned}
& \mathcal{I}_{\mathrm{D}_{1}}=\iint_{\Omega} \psi\left[\nabla \cdot\left(\kappa \nabla \delta_{\kappa} \phi\right)\right] d \Omega \\
& =\iint_{\Omega} \psi\left[\frac{\partial}{\partial x}\left(\kappa \frac{\partial \delta_{\kappa} \phi}{\partial x}\right)+\frac{\partial}{\partial y}\left(\kappa \frac{\partial \delta_{\kappa} \phi}{\partial y}\right)\right] d \Omega \\
& =\iint_{\Omega} \psi \frac{\partial}{\partial x}\left(\kappa \frac{\partial \delta_{\kappa} \phi}{\partial x}\right) d \Omega+\iint_{\Omega} \psi \frac{\partial}{\partial y}\left(\kappa \frac{\partial \delta_{\kappa} \phi}{\partial y}\right) d \Omega \\
& =\iint_{\Omega} \psi\left(\frac{\partial \kappa}{\partial x} \frac{\partial \delta_{\kappa} \phi}{\partial x}+\kappa \frac{\partial^{2} \delta_{\kappa} \phi}{\partial x^{2}}\right) d \Omega+\iint_{\Omega} \psi\left(\frac{\partial \kappa}{\partial y} \frac{\partial \delta_{\kappa} \phi}{\partial y}+\kappa \frac{\partial^{2} \delta_{\kappa} \phi}{\partial y^{2}}\right) d \Omega \\
& =\underbrace{\iint_{\Omega} \psi \frac{\partial \kappa}{\partial x} \frac{\partial \delta_{\kappa} \phi}{\partial x} d \Omega}_{\mathcal{I}_{\mathrm{D}_{1 \mathrm{~A}}}}+\underbrace{\iint_{\Omega} \psi \kappa \frac{\partial^{2} \delta_{\kappa} \phi}{\partial x^{2}} d \Omega}_{\mathcal{I}_{\mathrm{D}_{1 \mathrm{~B}}}}+\underbrace{\iint_{\Omega} \psi \frac{\partial \kappa}{\partial y} \frac{\partial \delta_{\kappa} \phi}{\partial y} d \Omega}_{\mathcal{I}_{\mathrm{D}_{1 \mathrm{C}}}}+\underbrace{\iint_{\Omega} \psi \kappa \frac{\partial^{2} \delta_{\kappa} \phi}{\partial y^{2}} d \Omega}_{\mathcal{I}_{\mathrm{D}_{1 \mathrm{D}}}}
\end{aligned}
$$

Aqui torna-se necessário uma nova divisão do processo em quatro integrais $\mathcal{I}_{\mathrm{D}_{1 \mathrm{~A}}}, \mathcal{I}_{\mathrm{D}_{1 \mathrm{~B}}}$, $\mathcal{I}_{\mathrm{D}_{1 \mathrm{C}}}$ e $\mathcal{I}_{\mathrm{D}_{1 \mathrm{D}}}$. No processo a seguir a técnica da integração por partes é novamente utilizada.

Cálculo de $\mathcal{I}_{\mathrm{D}_{1 \mathrm{~A}}}$ :

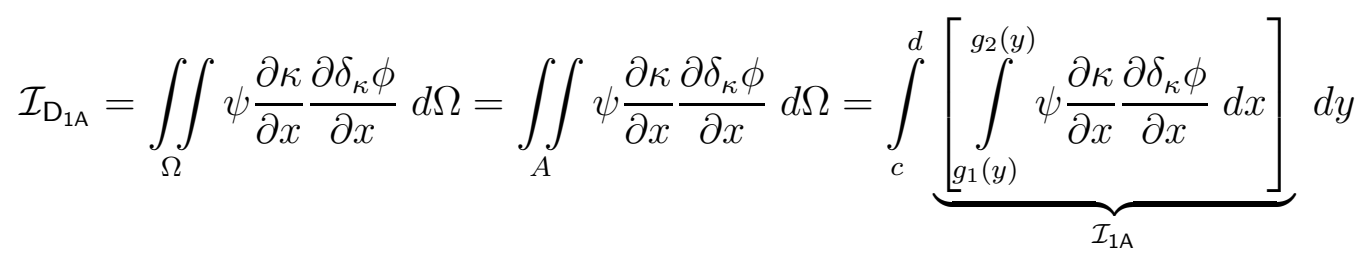

Integrando por partes $\mathcal{I}_{1 \mathrm{~A}}$, segue 


$$
\begin{aligned}
\mathcal{I}_{1 \mathrm{~A}}=\int_{g_{1}(y)}^{g_{2}(y)} \psi \frac{\partial \kappa}{\partial x} \frac{\partial \delta_{\kappa} \phi}{\partial x} d x=\left[\psi \frac{\partial \kappa}{\partial x} \delta_{\kappa} \phi\right]_{g_{1}(y)}^{g_{2}(y)}-\int_{g_{1}(y)}^{g_{2}(y)} \delta_{\kappa} \phi \frac{\partial}{\partial x}\left(\psi \frac{\partial \kappa}{\partial x}\right) d x \\
\\
\left\{\begin{array}{l}
p=\psi \frac{\partial \kappa}{\partial x} \Rightarrow d p=\frac{\partial}{\partial x}\left(\psi \frac{\partial \kappa}{\partial x}\right) d x \\
d q=\frac{\partial \delta_{\kappa} \phi}{\partial x} d x \Rightarrow q=\delta_{\kappa} \phi \\
\left.\mathcal{I}_{\mathrm{D}_{1 \mathrm{~A}}}=\int_{c}^{d}\left\{\psi \frac{\partial \kappa}{\partial x} \delta_{\kappa} \phi\right]_{g_{1}(y)}^{g_{2}(y)}-\int_{g_{1}(y)}^{g_{2}(y)} \delta_{\kappa} \phi \frac{\partial}{\partial x}\left(\psi \frac{\partial \kappa}{\partial x}\right) d x\right\} d y \\
=\int_{c}^{d}\left[\psi \frac{\partial \kappa}{\partial x} \delta_{\kappa} \phi\right]_{g_{1}(y)}^{g_{2}(y)} d y-\int_{c}^{g_{2}(y)} \int_{g_{1}(y)}^{\delta_{\kappa} \phi} \frac{\partial}{\partial x}\left(\psi \frac{\partial \kappa}{\partial x}\right) d x d y
\end{array}\right. \\
=
\end{aligned}
$$

Substituindo (3.29) em (3.28), obtém-se $\mathcal{I}_{\mathrm{D}_{1 \mathrm{~A}}}$, o qual é dado por

$$
\mathcal{I}_{\mathrm{D}_{1 \mathrm{~A}}}=\oint_{\partial \Omega} \psi \frac{\partial \kappa}{\partial x} \delta_{\kappa} \phi n_{x} d s-\iint_{\Omega} \delta_{\kappa} \phi \frac{\partial}{\partial x}\left(\psi \frac{\partial \kappa}{\partial x}\right) d \Omega
$$

Cálculo de $\mathcal{I}_{\mathrm{D}_{1 \mathrm{~B}}}$ : Essa integral envolve derivadas parciais de segunda ordem. Devido a isso, a técnica de integração por partes é aplicada duas vezes, como apresentado nos cálculos a seguir.

$$
\mathcal{I}_{\mathrm{D}_{1 \mathrm{~B}}}=\iint_{\Omega} \psi \kappa \frac{\partial^{2} \delta_{\kappa} \phi}{\partial x^{2}} d \Omega=\int_{c}^{d} \underbrace{\left[\int_{g_{1}(y)}^{g_{2}(y)} \psi \kappa \frac{\partial^{2} \delta_{\kappa} \phi}{\partial x^{2}} d x\right]}_{\mathcal{I}_{1 \mathrm{~B}}} d y
$$

Integrando por partes $\mathcal{I}_{1 \mathrm{~B}}$, segue

$$
\mathcal{I}_{1 \mathrm{~B}}=\int_{g_{1}(y)}^{g_{2}(y)} \psi \kappa \frac{\partial^{2} \delta_{\kappa} \phi}{\partial x^{2}} d x
$$




$$
\left\{\begin{array}{l}
p=\psi \kappa \Rightarrow d p=\left(\kappa \frac{\partial \psi}{\partial x}+\psi \frac{\partial \kappa}{\partial x}\right) d x \\
d q=\frac{\partial^{2} \delta_{\kappa} \phi}{\partial x^{2}} d x \Rightarrow q=\frac{\partial \delta_{\kappa} \phi}{\partial x}
\end{array}\right.
$$

resultando em

$$
\mathcal{I}_{1 \mathrm{~B}}=\left[\psi \kappa \frac{\partial \delta_{\kappa} \phi}{\partial x}\right]_{g_{1}(y)}^{g_{2}(y)}-\underbrace{\int_{g_{1}(y)}^{g_{2}(y)} \kappa \frac{\partial \psi}{\partial x} \frac{\partial \delta_{\kappa} \phi}{\partial x} d x}_{\mathcal{I}_{1 \mathrm{~B}_{1}}}-\underbrace{\int_{g_{1}(y)}^{g_{2}(y)} \psi \frac{\partial \kappa}{\partial x} \frac{\partial \delta_{\kappa} \phi}{\partial x} d x}_{\mathcal{I}_{1 \mathrm{~B}_{2}}}
$$

Os termos $\mathcal{I}_{1 \mathrm{~B}_{1}}$ e $\mathcal{I}_{1 \mathrm{~B}_{2}}$ em (3.33) precisam ser novamente integrados. Aplicando a técnica de integração por partes em $\mathcal{I}_{1 \mathrm{~B}_{1}}$ obtém-se

$$
\begin{gathered}
\mathcal{I}_{1 \mathrm{~B}_{1}}=\int_{g_{1}(y)}^{g_{2}(y)} \kappa \frac{\partial \psi}{\partial x} \frac{\partial \delta_{\kappa} \phi}{\partial x} d x=\left[\kappa \frac{\partial \psi}{\partial x} \delta_{\kappa} \phi\right]_{g_{1}(y)}^{g_{2}(y)}-\int_{g_{1}(y)}^{g_{2}(y)} \delta_{\kappa} \phi \frac{\partial}{\partial x}\left(\kappa \frac{\partial \psi}{\partial x}\right) d x \\
\left\{\begin{array}{l}
p=\kappa \frac{\partial \psi}{\partial x} \Rightarrow d p=\frac{\partial}{\partial x}\left(\kappa \frac{\partial \psi}{\partial x}\right) d x \\
d q=\frac{\partial \delta_{\kappa} \phi}{\partial x} d x \Rightarrow q=\delta_{\kappa} \phi
\end{array}\right.
\end{gathered}
$$

e para $\mathcal{I}_{1 \mathrm{~B}_{2}}$

$$
\begin{gathered}
\mathcal{I}_{1 \mathrm{~B}_{2}}=\int_{g_{1}(y)}^{g_{2}(y)} \psi \frac{\partial \kappa}{\partial x} \frac{\partial \delta_{\kappa} \phi}{\partial x} d x=\left[\psi \frac{\partial \kappa}{\partial x} \delta_{\kappa} \phi\right]_{g_{1}(y)}^{g_{2}(y)}-\int_{g_{1}(y)}^{g_{2}(y)} \delta_{\kappa} \phi \frac{\partial}{\partial x}\left(\psi \frac{\partial \kappa}{\partial x}\right) d x \\
\left\{\begin{array}{l}
p=\psi \frac{\partial \kappa}{\partial x} \Rightarrow d p=\frac{\partial}{\partial x}\left(\psi \frac{\partial \kappa}{\partial x}\right) d x \\
d q=\frac{\partial \delta_{\kappa} \phi}{\partial x} d x \Rightarrow q=\delta_{\kappa} \phi
\end{array}\right.
\end{gathered}
$$


Substituindo os resultados (3.34) e (3.35) em (3.33), segue

$$
\begin{gathered}
\mathcal{I}_{1 \mathrm{~B}}=\left[\psi \kappa \frac{\partial \delta_{\kappa} \phi}{\partial x}\right]_{g_{1}(y)}^{g_{2}(y)}-\left[\kappa \frac{\partial \psi}{\partial x} \delta_{\kappa} \phi\right]_{g_{1}(y)}^{g_{2}(y)}+\int_{g_{1}(y)}^{g_{2}(y)} \delta_{\kappa} \phi \frac{\partial}{\partial x}\left(\kappa \frac{\partial \psi}{\partial x}\right) d x- \\
\mathcal{I}_{1 \mathrm{~B}}=\left[\psi \kappa \frac{\partial \kappa}{\partial x} \delta_{\kappa} \phi\right]_{g_{1}(y)}^{g_{2}(y)}+\int_{g_{1}(y)}^{g_{2}(y)} \delta_{\kappa} \phi \frac{\partial}{\partial x}\left(\psi \frac{\partial \psi}{\partial x} \delta_{\kappa} \phi-\psi \frac{\partial \kappa}{\partial x} \delta_{\kappa} \phi\right]_{g_{1}(y)}^{g_{2}(y)}+\int_{g_{1}(y)}^{g_{2}(y)} \delta_{\kappa} \phi \frac{\partial}{\partial x}\left(\kappa \frac{\partial \psi}{\partial x}+\psi \frac{\partial \kappa}{\partial x}\right) d x \\
\mathcal{I}_{1 \mathrm{~B}}=\left[\psi \kappa \frac{\partial \delta_{\kappa} \phi}{\partial x}-\delta_{\kappa} \phi \frac{\partial(\kappa \psi)}{\partial x}\right]_{g_{1}(y)}^{g_{2}(y)}+\int_{g_{1}(y)}^{g_{2}(y)} \delta_{\kappa} \phi \frac{\partial}{\partial x}\left[\frac{\partial(\kappa \psi)}{\partial x}\right] d x
\end{gathered}
$$

Substituindo (3.36) em (3.31), obtém-se

$$
\begin{gathered}
\mathcal{I}_{\mathrm{D}_{1 \mathrm{~B}}}=\int_{c}^{d}\left\{\left[\psi \kappa \frac{\partial \delta_{\kappa} \phi}{\partial x}-\delta_{\kappa} \phi \frac{\partial(\kappa \psi)}{\partial x}\right]_{g_{1}(y)}^{g_{2}(y)}+\int_{g_{1}(y)}^{g_{2}(y)} \delta_{\kappa} \phi \frac{\partial}{\partial x}\left[\frac{\partial(\kappa \psi)}{\partial x}\right] d x\right\} d y \\
\mathcal{I}_{\mathrm{D}_{1 \mathrm{~B}}}=\int_{c}^{d}\left[\psi \kappa \frac{\partial \delta_{\kappa} \phi}{\partial x}-\delta_{\kappa} \phi \frac{\partial(\kappa \psi)}{\partial x}\right]_{g_{1}(y)}^{g_{2}(y)} d y+\int_{c}^{d} \int_{g_{1}(y)}^{g_{2}(y)} \delta_{\kappa} \phi \frac{\partial}{\partial x}\left[\frac{\partial(\kappa \psi)}{\partial x}\right] d x d y
\end{gathered}
$$

Com isso, $\mathcal{I}_{\mathrm{D}_{1 \mathrm{~B}}}$ é dado por

$$
\mathcal{I}_{\mathrm{D}_{1 \mathrm{~B}}}=\oint_{\partial \Omega}\left[\psi \kappa \frac{\partial \delta_{\kappa} \phi}{\partial x}-\delta_{\kappa} \phi \frac{\partial(\kappa \psi)}{\partial x}\right] n_{x} d s+\iint_{\Omega} \delta_{\kappa} \phi \frac{\partial}{\partial x}\left[\frac{\partial(\kappa \psi)}{\partial x}\right] d \Omega
$$

Cálculo de $\mathcal{I}_{\mathrm{D}_{1 \mathrm{C}}}$ : 
$\mathrm{O}$ cálculo de $\mathcal{I}_{1 \mathrm{C}}$ é análogo ao feito para $\mathcal{I}_{1 \mathrm{~A}}$. Daí, temos

$$
\mathcal{I}_{\mathrm{D}_{1 \mathrm{C}}}=\oint_{\partial \Omega} \psi \frac{\partial \kappa}{\partial y} \delta_{\kappa} \phi n_{y} d s-\iint_{\Omega} \delta_{\kappa} \phi \frac{\partial}{\partial y}\left(\psi \frac{\partial \kappa}{\partial y}\right) d \Omega
$$

Cálculo de $\mathcal{I}_{\mathrm{D}_{1 \mathrm{D}}}$ :

$\mathrm{O}$ cálculo de $\mathcal{I}_{1 \mathrm{D}}$ é análogo ao feito para $\mathcal{I}_{1 \mathrm{~B}}$. Daí, temos

$$
\mathcal{I}_{\mathrm{D}_{1 \mathrm{D}}}=\oint_{\partial \Omega}\left[\psi \kappa \frac{\partial \delta_{\kappa} \phi}{\partial y}-\delta_{\kappa} \phi \frac{\partial(\kappa \psi)}{\partial y}\right] n_{y} d s+\iint_{\Omega} \delta_{\kappa} \phi \frac{\partial}{\partial y}\left[\frac{\partial(\kappa \psi)}{\partial y}\right] d \Omega
$$

Somando os resultados de cada uma das quatro integrais (3.30), (3.37), (3.38) e (3.39), segue

$$
\begin{aligned}
\mathcal{I}_{\mathrm{D}_{1}}=\oint_{\partial \Omega} \psi \frac{\partial \kappa}{\partial x} \delta_{\kappa} \phi n_{x} d s-\iint_{\Omega} \delta_{\kappa} \phi \frac{\partial}{\partial x}\left(\psi \frac{\partial \kappa}{\partial x}\right) d \Omega+ \\
\oint_{\partial \Omega}\left[\psi \kappa \frac{\partial \delta_{\kappa} \phi}{\partial x}-\delta_{\kappa} \phi \frac{\partial(\kappa \psi)}{\partial x}\right] n_{x} d s+\iint_{\Omega} \delta_{\kappa} \phi \frac{\partial}{\partial x}\left[\frac{\partial(\kappa \psi)}{\partial x}\right] d \Omega+ \\
\oint_{\partial \Omega} \psi \frac{\partial \kappa}{\partial y} \delta_{\kappa} \phi n_{y} d s-\iint_{\Omega} \delta_{\kappa} \phi \frac{\partial}{\partial y}\left(\psi \frac{\partial \kappa}{\partial y}\right) d \Omega+ \\
\oint_{\partial \Omega}\left[\psi \kappa \frac{\partial \delta_{\kappa} \phi}{\partial y}-\delta_{\kappa} \phi \frac{\partial(\kappa \psi)}{\partial y}\right] n_{y} d s+\iint_{\Omega} \delta_{\kappa} \phi \frac{\partial}{\partial y}\left[\frac{\partial(\kappa \psi)}{\partial y}\right] d \Omega
\end{aligned}
$$

$\mathcal{I}_{\mathrm{D}_{1}}=\oint_{\partial \Omega}\left\{\delta_{\kappa} \phi[\psi \nabla \kappa-\nabla(\kappa \psi)]+\kappa \psi \nabla \delta_{\kappa} \phi\right\} \cdot n d s+\iint_{\Omega}\left\{\delta_{\kappa} \phi\left[\nabla^{2}(\kappa \psi)-\nabla \cdot(\psi \nabla \kappa)\right]\right\} d \Omega$

Para obter $\mathcal{I}_{\mathrm{D}_{2}}$, as operações são análogas as feitas para obter $\mathcal{I}_{\mathrm{D}_{1}}$. Com isso, temos

$$
\mathcal{I}_{\mathrm{D}_{2}}=\oint_{\partial \Omega}\{\phi[\psi \nabla \delta \kappa-\nabla(\delta \kappa \psi)]+\delta \kappa \psi \nabla \phi\} \cdot n d s+\iint_{\Omega}\left\{\phi\left[\nabla^{2}(\delta \kappa \psi)-\nabla \cdot(\psi \nabla \delta \kappa)\right]\right\} d \Omega
$$


Substituindo (3.41) e (3.42) em (3.27), obtém-se a integral da parte difusiva $\mathcal{I}_{\mathrm{D}}$, a qual é dada por

$$
\begin{aligned}
\mathcal{I}_{\mathrm{D}}=\oint_{\partial \Omega}\left\{\delta_{\kappa} \phi[\psi \nabla \kappa-\nabla(\kappa \psi)]+\kappa \psi \nabla \delta_{\kappa} \phi\right\} \cdot n d s+\iint_{\Omega}\left\{\delta_{\kappa} \phi\left[\nabla^{2}(\kappa \psi)-\nabla \cdot(\psi \nabla \kappa)\right]\right\} d \Omega+ \\
\oint_{\partial \Omega}\{\phi[\psi \nabla \delta \kappa-\nabla(\delta \kappa \psi)]+\delta \kappa \psi \nabla \phi\} \cdot n d s+\iint_{\Omega}\left\{\phi\left[\nabla^{2}(\delta \kappa \psi)-\nabla \cdot(\psi \nabla \delta \kappa)\right]\right\} d \Omega
\end{aligned}
$$

Logo, somando $\mathcal{I}_{\mathrm{A}}(3.26)$, a integral da parte advectiva, com $\mathcal{I}_{\mathrm{D}}$ (3.43), a integral da parte difusiva, obtem-se $\mathcal{I}_{\mathrm{AD}}$, a soma das integrais da parte advectiva e difusiva, a qual é dada por

$$
\begin{aligned}
& \mathcal{I}_{\mathrm{AD}}=\oint_{\partial \Omega} \psi \delta_{\kappa} \phi \vec{\omega} \cdot n d s-\iint_{\Omega} \delta_{\kappa} \phi \vec{\omega} \cdot \nabla \psi d \Omega- \\
& \oint_{\partial \Omega}\left\{\delta_{\kappa} \phi[\psi \nabla \kappa-\nabla(\kappa \psi)]+\kappa \psi \nabla \delta_{\kappa} \phi\right\} \cdot n d s-\iint_{\Omega}\left\{\delta_{\kappa} \phi\left[\nabla^{2}(\kappa \psi)-\nabla \cdot(\psi \nabla \kappa)\right]\right\} d \Omega- \\
& \oint_{\partial \Omega}\{\phi[\psi \nabla \delta \kappa-\nabla(\delta \kappa \psi)]+\delta \kappa \psi \nabla \phi\} \cdot n d s-\iint_{\Omega}\left\{\phi\left[\nabla^{2}(\delta \kappa \psi)-\nabla \cdot(\psi \nabla \delta \kappa)\right]\right\} d \Omega \\
& \mathcal{I}_{\mathrm{AD}}=\int_{\Omega} \delta_{\kappa} \phi\{-\vec{\omega} \cdot \nabla \psi-\nabla \cdot[\nabla(\kappa \psi)-\psi \nabla \kappa]\} d \Omega+\oint_{\partial \Omega}\{\phi[\psi \nabla \delta \kappa-\nabla(\delta \kappa \psi)]+\delta \kappa \psi \nabla \phi\} \cdot n d s- \\
& \oint_{\partial \Omega}\left\{\delta_{\kappa} \phi[\psi(\vec{\omega}-\nabla \kappa)+\nabla(\kappa \psi)]-\kappa \psi \nabla \delta_{\kappa} \phi\right\} \cdot n d s-\int_{\Omega}\left\{\phi\left[\nabla^{2}(\delta \kappa \psi)-\nabla \cdot(\psi \nabla \delta \kappa)\right]\right\} d \Omega \text { (3.44) }
\end{aligned}
$$

\subsubsection{Equação adjunta}

Voltando agora ao gradiente $\delta_{\kappa} \mathcal{F}$, substituindo (3.44) em (3.15), segue 


$$
\begin{aligned}
& \delta_{\kappa} \mathcal{F}=\iint_{\Omega}\left(\phi-\phi_{\mathrm{R}}\right) \delta_{\kappa} \phi d \Omega+ \\
& \iint_{\Omega} \delta_{\kappa} \phi\{-\vec{\omega} \cdot \nabla \psi-\nabla \cdot[\nabla(\kappa \psi)-\psi \nabla \kappa]\} d \Omega+ \\
& \oint_{\partial \Omega}\left\{\delta_{\kappa} \phi[\psi(\vec{\omega}-\nabla \kappa)+\nabla(\kappa \psi)]-\kappa \psi \nabla \delta_{\kappa} \phi\right\} \cdot n d s- \\
& \oint_{\partial \Omega}\{\phi[\psi \nabla \delta \kappa-\nabla(\delta \kappa \psi)]+\delta \kappa \psi \nabla \phi\} \cdot n d s- \\
& \iint_{\Omega}\left\{\phi\left[\nabla^{2}(\delta \kappa \psi)-\nabla \cdot(\psi \nabla \delta \kappa)\right]\right\} d \Omega \\
& \delta_{\kappa} \mathcal{F}=\iint_{\Omega} \delta_{\kappa} \phi\left\{-\vec{\omega} \cdot \nabla \psi-\nabla \cdot[\nabla(\kappa \psi)-\psi \nabla \kappa]+\left(\phi-\phi_{\mathrm{R}}\right)\right\} d \Omega+ \\
& \oint_{\partial \Omega}\left\{\delta_{\kappa} \phi[\psi(\vec{\omega}-\nabla \kappa)+\nabla(\kappa \psi)]-\kappa \psi \nabla \delta_{\kappa} \phi\right\} \cdot n d s- \\
& \oint_{\partial \Omega}\{\phi[\psi \nabla \delta \kappa-\nabla(\delta \kappa \psi)]+\delta \kappa \psi \nabla \phi\} \cdot n d s- \\
& \iint_{\Omega}\left\{\phi\left[\nabla^{2}(\delta \kappa \psi)-\nabla \cdot(\psi \nabla \delta \kappa)\right]\right\} d \Omega
\end{aligned}
$$




$$
\begin{aligned}
\delta_{\kappa} \mathcal{F}= & \iint_{\Omega} \delta_{\kappa} \phi\left\{-\vec{\omega} \cdot \nabla \psi-\nabla \cdot(\kappa \nabla \psi)+\left(\phi-\phi_{\mathrm{R}}\right)\right\} d \Omega+ \\
& \oint_{\partial \Omega}\left\{\delta_{\kappa} \phi[\vec{\omega} \psi+\kappa \nabla \psi]-\kappa \psi \nabla \delta_{\kappa} \phi\right\} \cdot n d s+ \\
& \oint_{\partial \Omega}\{\delta \kappa[\phi \nabla \psi-\psi \nabla \phi]\} \cdot n d s- \\
& \int_{\Omega}\{\phi \nabla \cdot(\delta \kappa \nabla \psi)\} d \Omega
\end{aligned}
$$

No cálculo dos gradientes $\delta_{\kappa} \mathcal{F}$, não há interesse que os mesmos dependam dos cálculos das variações $\delta_{\kappa} \phi$. Para tirar essa dependência, basta impor que

$$
-\vec{\omega} \cdot \nabla \psi-\nabla \cdot(\kappa \nabla \psi)+\left(\phi-\phi_{\mathrm{R}}\right)=0 \Rightarrow-\vec{\omega} \cdot \nabla \psi-\nabla \cdot(\kappa \nabla \psi)=\phi_{\mathrm{R}}-\phi
$$

\section{A equação}

$$
-\vec{\omega} \cdot \nabla \psi-\nabla \cdot(\kappa \nabla \psi)=\phi_{\mathrm{R}}-\phi
$$

define a chamada equação adjunta ou equação dual. Perceba que em (3.45) ainda resta uma integral de linha no contorno do domínio contendo $\delta_{\kappa} \phi$.

$$
\oint_{\partial \Omega}\left\{\delta_{\kappa} \phi[\vec{\omega} \psi+\kappa \nabla \psi]-\kappa \psi \nabla \delta_{\kappa} \phi\right\} \cdot n d s
$$

É com essa integral e as condições de fronteira (3.12) da equação de sensibilidade (??) , que são obtidas as condições de fronteira da equação adjunta. Na parte do contorno que $\delta_{\kappa} \phi$ se anula, devemos impor $\psi=0$, e na parte onde $\nabla \delta_{\kappa} \phi=0$, devemos impor $\vec{\omega} \psi+\kappa \nabla \psi=0$. Dessa forma, podemos obter os gradientes $\delta_{\kappa} \mathcal{F}$ 
sem a necessidade de calcular $\delta_{\kappa} \phi$. Em cada iteração do processo de otimização é necessário calcular uma solução primal e uma solução adjunta para obter os gradientes.

Uma observação importante deve ser feita aqui. Note que

$$
\nabla \cdot(\vec{\omega} \psi)=\vec{\omega} \cdot \nabla \psi+\psi \nabla \cdot \vec{\omega} \Rightarrow-\vec{\omega} \cdot \nabla \psi=-\nabla \cdot(\vec{\omega} \psi)+\psi \nabla \cdot \vec{\omega}
$$

Nesse trabalho, os testes são feitos considerando $\vec{\omega}$ conhecido em todo domínio e o escoamento incompressível. Logo, o divergente $\nabla \cdot \vec{\omega}=0$. Daí, segue-se

$$
-\vec{\omega} \cdot \nabla \psi=-\nabla \cdot(\vec{\omega} \psi)
$$

Substituindo (3.48) na equação adjunta (3.46), obtém-se

$$
-\nabla \cdot(\vec{\omega} \psi)-\nabla \cdot(\kappa \nabla \psi)=\phi_{\mathrm{R}}-\phi
$$

A equação adjunta utilizada então é dada por (3.49). A equação nessa forma tem a vantagem de apresentar a forma conservativa.

Os gradientes são calculados usando dos termos restantes em (3.45)

$$
\delta_{\kappa} \mathcal{F}=\oint_{\partial \Omega}\{\delta \kappa[\phi \nabla \psi-\psi \nabla \phi]\} \cdot n d s-\iint_{\Omega}\{\phi \nabla \cdot(\delta \kappa \nabla \psi)\} d \Omega
$$

Um detalhe que pode ser observado tanto no problema primal como no problema adjunto é que na implementação, tanto o problema primal como o adjunto utilizam a mesma máquina de solução. Não é necessário utilizar métodos distintos.

A próxima seção faz a apresentação do problema de teste modelo utilizado nesse trabalho.

\subsection{Problema de teste}

O problema de teste é um problema de identificação de parâmetros, está inserido em um problema de otimização, o qual consiste em estimar os coeficientes $\beta_{k}$ na expressão 


$$
\kappa(x, y)=C_{0}+\sum_{\mathrm{k}=1}^{\mathrm{N}} \beta_{\mathrm{k}} C_{\mathrm{k}}(x, y)
$$

sendo $C_{0}$ uma constante, $C_{\mathrm{k}}(x, y)$ funções previamente escolhidas, de tal forma que minimize o funcional

$$
\mathcal{F}[\kappa]=\frac{1}{2} \iint_{\Omega}\left(\phi-\phi_{\mathrm{R}}\right)^{2} d \Omega
$$

restrito a equação de advecção-difusão

$$
\nabla \cdot(\vec{\omega} \phi)-\nabla(\kappa \nabla \phi)=0 \quad(x, y) \in[0,1] \times[0,1] \quad \vec{\omega}=(1,0)
$$

definida em um quadrado unitário, sendo as condições de fronteiras dadas por

$$
\begin{aligned}
& \phi(0, y)= \begin{cases}1, & 0 \leq y \leq 0.4 \\
5 y-1, & 0.4<y<0.6 \\
2, & 0.6 \leq y \leq 1.0\end{cases} \\
& \phi(x, 0)=1 \quad 0<x \leq 1 \\
& \phi(x, 1)=2 \quad 0<x \leq 1 \\
& \frac{\partial \phi(1, y)}{\partial n}=0 \quad 0<y<1
\end{aligned}
$$

Considere agora a formulação das condições de fronteira do problema adjunto (ou problema dual) para esse problema teste. Na seção anterior, para montar as condições de fronteira do problema adjunto, foi explicado que na parte do contorno onde $\delta_{\kappa} \phi$ se anula, devemos impor $\psi=0$, e na parte onde $\nabla \delta_{\kappa} \phi=0$, devemos impor $\vec{\omega} \psi+\kappa \nabla \psi=0$. Perceba que as condições de fronteira para equação de sensibilidade desse problema teste são dadas por

$$
\delta_{\kappa} \phi(0, y)= \begin{cases}0, & 0 \leq y \leq 0.4 \\ 0, & 0.4<y<0.6 \\ 0, & 0.6 \leq y \leq 1.0\end{cases}
$$




$$
\begin{array}{cl}
\delta_{\kappa} \phi(x, 0)=0 & 0<x \leq 1 \\
\delta_{\kappa} \phi(x, 1)=0 & 0<x \leq 1 \\
\frac{\partial \delta_{\kappa} \phi(1, y)}{\partial n}=0 & 0<y<1
\end{array}
$$

Assim sendo, analisando (3.56), (3.57), (3.58) e (3.59), segue que as condições de fronteira para o problema adjunto são dadas por

$$
\begin{aligned}
& \psi(0, y)= \begin{cases}0, & 0 \leq y \leq 0.4 \\
0, & 0.4<y<0.6 \\
0, & 0.6 \leq y \leq 1.0\end{cases} \\
& \psi(x, 0)=0 \quad 0<x \leq 1 \\
& \psi(x, 1)=0 \quad 0<x \leq 1 \\
& {[\vec{\omega} \psi+\kappa \nabla \psi] \cdot n=0 \quad x=1 \quad \text { e } \quad 0<y<1}
\end{aligned}
$$

A próxima seção descreve como são feitos os cálculos das integrais presentes na função objetivo e na fórmula do gradiente.

\subsection{Cálculo das integrais}

O funcional

$$
\mathcal{F}[\kappa]=\frac{1}{2} \iint_{\Omega}\left(\phi-\phi_{\mathrm{R}}\right)^{2} d \Omega
$$

e os gradientes 


$$
\delta_{\kappa} \mathcal{F}=\oint_{\partial \Omega}\{\delta \kappa[\phi \nabla \psi-\psi \nabla \phi]\} \cdot n d s-\iint_{\Omega}\{\phi \nabla \cdot(\delta \kappa \nabla \psi)\} d \Omega
$$

exigem cálculo de integrais para serem avaliadas. Para calcular o funcional integral, primeiro faz-se a reconstrução de $\left(\phi-\phi_{\mathrm{R}}\right)^{2}$, uma vez que ambas as parcelas nessas diferenças são conhecidas em todos os vértices. Tendo obtido a reconstrução, que são polinômios, estes são integrados utilizando a técnica de integração, baseado em quadratura gaussiana, que utiliza 13 pontos [12], e foi apresentada no segundo capítulo. Para calcular as integrais de área que aparece no gradiente, utiliza-se a mesma idéia. Emprega-se o mesmo esquema. Já para integral de linha, utilizam-se as reconstruções de $\phi$ e $\psi$, os pontos de Gauss e pesos, sendo que estes dois últimos (pontos e pesos) também constam no segundo capítulo. Os termos $\delta \kappa$ que aparecem, considerando a forma funcional dos coeficientes de difusão tratados nesse trabalho,

$$
\kappa(x, y)=C_{0}+\sum_{\mathrm{k}=1}^{\mathrm{N}} \beta_{k} C_{\mathrm{k}}(x, y)
$$

de uma forma generalizada, são dados por

$$
\delta_{\beta_{\mathrm{k}}} \kappa=C_{\mathrm{k}}(x, y)
$$

Para calcular essas variações, utiliza-se o mesmo princípio para calcular $\delta_{\kappa} \mathcal{F}$.

$$
\delta_{\beta_{\mathrm{k}}} \kappa=\lim _{\varepsilon \rightarrow 0} \frac{\kappa\left(\beta_{\mathrm{k}}+\delta \beta_{\mathrm{k}}\right)-\kappa\left(\beta_{\mathrm{k}}\right)}{\varepsilon}
$$

O próximo capítulo apresenta um conjunto de testes feitos envolvendo estimação de parâmetros, no caso, para determinar os parâmetros que define a função coeficiente de difusão na forma

$$
\kappa(x, y)=C_{0}+\sum_{\mathrm{k}=1}^{\mathrm{N}} \beta_{\mathrm{k}} C_{\mathrm{k}}(x, y)
$$

onde $C_{0} \neq 0$ é uma constante fixa e $C_{\mathrm{k}}(x, y), \mathrm{k}=1, \ldots, \mathrm{N}$, são funções previamente estabelecidas. 


\section{Estudo numérico do problema de identificação de parâmetros}

Esse capítulo tem por finalidade descrever, bem como apresentar os resultados obtidos, no estudo do problema de identificação de parâmetros. A primeira seção apresenta, em linhas gerais, o que precisa ser calculado durante a resolução numérica do problema de otimização, além de alguns detalhes no que tange aos objetivos desse estudo. A segunda seção apresenta o algoritmo utilizado no problema de otimização em seus detalhes. As demais seções apresentam os testes realizados, bem como a análise dos resultados.

\subsection{Introdução}

Como comentado no primeiro capítulo, o problema de identificação de parâmetros utiliza um problema de otimização com restrição, baseado em gradiente, como ferramenta para estimar os parâmetros. Assim sendo, dado um método de otimização, a função objetivo e o gradiente precisam ser avaliados em cada iteração. No caso desse trabalho, para cada conjunto de parâmetros $\beta_{\mathrm{k}}, \mathrm{k}=1, \ldots, \mathrm{N}$, que definem a função coeficiente de difusão, é necessário, para avaliar a função objetivo, resolver o problema primal e calcular uma integral de área. Para obter os gradientes, é necessário resolver o problema adjunto utilizando a solução do problema primal, a qual é necessária para determinar o termo fonte do problema adjunto, e calcular integrais de área e de linha. Note também que as soluções do problema primal e do adjunto são dependentes dos parâmetros $\beta_{\mathrm{k}}$. A figura 4.1 descreve essas relações de dependência.

Uma seqüência de procedimentos, baseada nessas relações de dependência, é levada a efeito para obter os parâmetros. O método de otimização utilizado é o método dos gradientes conjugados (MGC) [32]. O MGC realiza as pesquisas de busca nas direções conjugadas aos gradientes. Nesse método, dado um ponto e uma direção de busca, no qual a função objetivo é decrescente, em cada iteração deve ser dado um passo na referida direção. Para obter o tamanho desse passo, é utilizado o método do segmento aúreo (Golden 


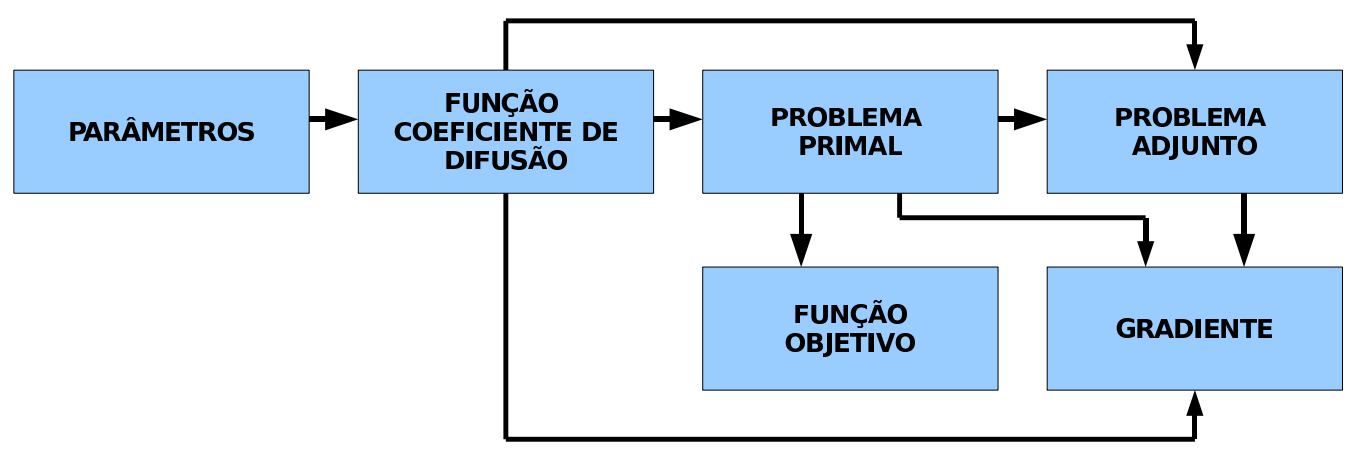

Figura 4.1: Relações de dependência

Search) [32]. O critério de parada é baseado na norma da função objetivo juntamente com um número máximo de iterações. Esse critério foi utilizado por que as soluções de referência $\phi_{\mathrm{R}}$ foram calculadas a partir de parâmetros de referência $\beta_{\mathrm{k}}^{\mathrm{R}}$.

No processo de identificação dos parâmetros, o método dos gradientes conjugados é uma capa externa em todo processo. As rotinas de cálculo da função objetivo e das componentes do vetor gradiente são internas ao MGC. Assim sendo, o MGC controla todo processo de busca e dentro dele ficam as rotinas para avaliar a função objetivo e os gradientes, os quais são chamados sempre que necessário.

A próxima seção apresenta detalhamentos com relação ao algoritmo utilizado para estimar os parâmetros.

\subsection{Descrição do algoritmo}

O algoritmo exige a resolução de duas EDPs, problemas primal e adjunto, e o cálculo de integrais de área e linha para avaliar a função objetivo e o gradiente. Cada uma dessas EDPs e integrais são dadas por:

\section{- Problema primal:}

$$
\nabla \cdot(\vec{\omega} \phi)-\nabla \cdot(\kappa \nabla \phi)=0 \quad(x, y) \in \Omega \subset \mathbb{R}^{2}
$$

com condições de fronteira

$$
\begin{cases}\phi=f, & (x, y) \in \partial \Omega_{1} \\ \nabla \phi \cdot n=g, & (x, y) \in \partial \Omega_{2}\end{cases}
$$

sendo $\omega=(u, v)$ conhecido. Essa equação é resolvida utilizando o método dos volumes finitos, a técnica de reconstrução de alta ordem (para calcular os fluxos advectivos e difusivos) e o método de Runge-Kutta multiestágio, conforme foi descrito no segundo capítulo. 


\section{- Problema adjunto:}

$$
-\nabla \cdot(\omega \psi)-\nabla \cdot(\kappa \nabla \psi)=\phi_{\mathrm{R}}-\phi \quad(x, y) \in \Omega \subset \mathbb{R}^{2}
$$

com condições de fronteira

$$
\begin{cases}\psi=0, & (x, y) \in \partial \Omega_{1} \\ {[\vec{\omega} \psi+\kappa \nabla \psi] \cdot n=0,} & (x, y) \in \partial \Omega_{2}\end{cases}
$$

sendo $\vec{\omega}=(u, v)$ conhecido. Essa equação é resolvida utilizando o mesmo método utilizado para o resolver o problema primal.

- Função objetivo:

$$
\mathcal{F}[\phi(\beta)]=\frac{1}{2} \iint_{\Omega}\left(\phi-\phi_{\mathrm{R}}\right)^{2} d \Omega
$$

\section{- Gradiente:}

- Via método adjunto:

$$
\delta_{\beta_{\mathrm{k}}} \mathcal{F}=\oint_{\partial \Omega}\left\{\delta_{\beta_{\mathrm{k}}} \kappa[\phi \nabla \psi-\psi \nabla \phi]\right\} \cdot n d s-\iint_{\Omega}\left\{\phi \nabla \cdot\left(\delta_{\beta_{\mathrm{k}}} \kappa \nabla \psi\right)\right\} d \Omega \quad \mathrm{k}=1, \ldots, \mathrm{N}
$$

Em (4.2) o que se tem são as componentes do vetor gradiente. Além disso, os termos $\delta_{\beta_{k}} \kappa$ são dados por $\delta_{\beta_{\mathrm{k}}} \kappa=C_{\mathrm{k}}(x, y)$, onde $C_{\mathrm{k}}$ são as funções previamente definidas no coeficiente difusão $\kappa(x, y)$.

\section{- Via diferenças finitas:}

$$
\delta_{\beta_{\mathrm{k}}} \mathcal{F}=\frac{\mathcal{F}\left[\phi\left(\beta_{\mathrm{k}}+\delta \beta_{\mathrm{k}}\right)\right]-\mathcal{F}\left[\phi\left(\beta_{\mathrm{k}}\right)\right]}{\delta \beta_{k}} \quad \mathrm{k}=1,2, \ldots, \mathrm{N}
$$

Em (4.3) também o que se tem são as componentes do vetor gradiente. Para calcular o gradiente em relação a um determinado $\beta_{\mathrm{k}}$, deve-se avaliar $\mathcal{F}$ para os valores dos $\mathrm{N}$ parâmetros $\beta_{\mathrm{k}}$ da última iteração. Depois disso, deve-se pertubar cada $\beta_{\mathrm{k}}$ por um valor $\delta \beta_{\mathrm{k}}$ (valor pequeno), manter os demais parâmetros inalterados, avaliar $\mathcal{F}$ e calcular a razão em (4.3). Essa pertubação deve ser feita parâmetro a parâmetro para obter todas as componentes do vetor gradiente. 
Reiterando o que foi descrito na introdução desse capítulo, para avaliar a função objetivo (4.1) é necessário resolver o problema primal para um determinado conjunto formado pelos $\mathrm{N}$ parâmetros $\beta_{\mathrm{k}}$, e em seguida calcular uma integral de área. Para calcular os gradientes (4.2) é necessário além da solução do problema primal, para obter o termo fonte do problema adjunto, resolver o problema adjunto e calcular uma integral de área e outra de linha. A integral da função objetivo é avaliada de uma única vez para o domínio todo a cada vez que é executada. No caso do gradiente, uma integral de área e outra de linha tem que ser avaliada para cada parâmetro $\beta_{\mathrm{k}}$. Nas explicações a seguir, o gradiente é denotado por $\nabla \mathcal{F}$.

O fluxograma 4.2 apresenta os passos necessários para estimar, utilizando o método dos gradientes conjugados, os parâmetros $\beta_{\mathrm{k}}$ em

$$
\kappa(x, y)=C_{0}+\sum_{\mathrm{k}=1}^{\mathrm{N}} \beta_{\mathrm{k}} C_{\mathrm{k}}(x, y)
$$

onde $C_{0} \neq 0$ é uma constante fixa e $C_{\mathrm{k}}(x, y), \mathrm{k}=1, \ldots, \mathrm{N}$, são funções previamente estabelecidas. Segue detalhamento de cada um dos passos do fluxograma:

(1)

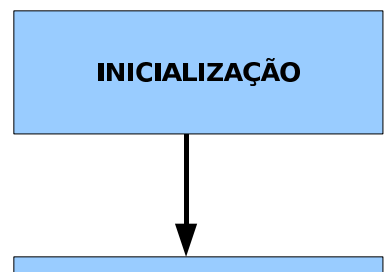

(2)

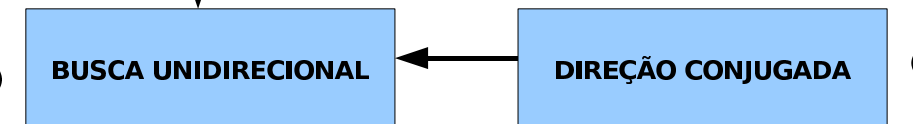

(5)

(3)
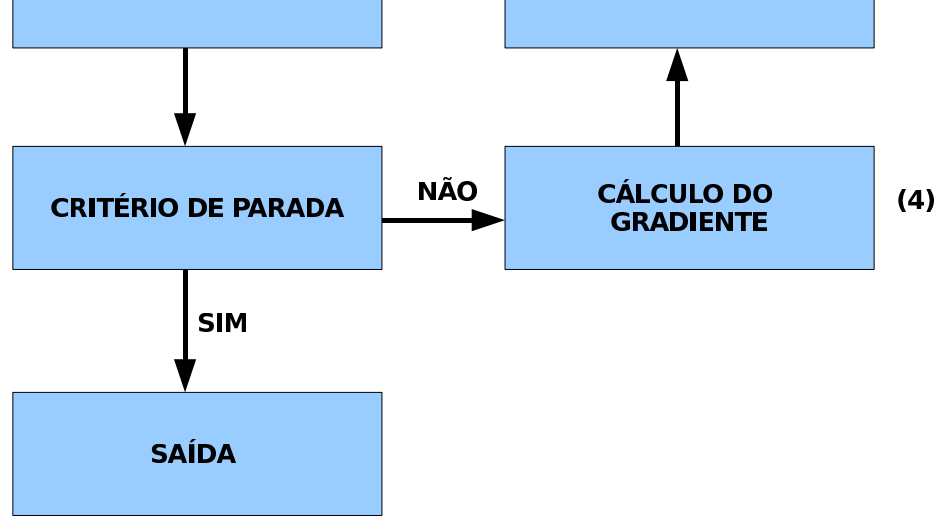

Figura 4.2: Fluxograma para o problema de identificação de parâmetros utilizando o método dos gradientes conjugados

- (1) Inicialização: Nesse estágio, definem-se: 
1. Um campo de valores da propriedade transportada $\phi_{\mathrm{R}}$ numa região onde se deseje estimar os $\mathrm{N}$ coeficientes $\beta_{\mathrm{k}}$ que definem a função coeficiente de difusão (4.4).

2. Uma aproximação inicial $\beta_{\mathrm{k}}^{(0)}$ para os $\mathrm{N}$ parâmetros.

3. Uma aproximação inicial para o vetor gradiente $\nabla \mathcal{F}\left(\beta^{(0)}\right)$, onde $\beta_{\mathrm{k}}^{(0)}$ são os $\mathrm{N}$ parâmetros iniciais. Chame $g^{(0)}=\nabla \mathcal{F}\left(\beta^{(0)}\right)$ e $s^{(0)}=-g^{(0)}$.

Observações: Para definir esse campo inicial $\phi_{\mathrm{R}}$, pode-se utilizar $\mathrm{N}$ parâmetros de referência $\beta_{\mathrm{k}}^{\mathrm{R}}$ e rodar o problema primal para obter $\phi_{\mathrm{R}}$. Essa estratégia foi utilizada nos testes desse trabalho com fins de estudar o efeito das técnicas utilizadas, as quais foram comentadas na primeira seção desse capítulo.

- (2) Busca unidirecional: A busca unidirecional consiste em realizar uma busca na direção conjugada ao gradiente, direção esta onde a função objetivo é decrescente. Existe um ponto no caminho de busca onde a função objetivo $\mathcal{F}$ assume o menor valor. Para ir até esse ponto, é necessário dar um passo na direção do gradiente conjugado utilizando a relação $\beta^{(\mathrm{n}+1)}=\beta^{(\mathrm{n})}+\alpha_{n} s^{(\mathrm{n})}$, onde $\alpha_{n}$ é o tamanho do passo na direção de pesquisa e $\beta$ é o vetor dos parâmetros, o qual tem dimensão igual a $\mathrm{N}$.

Para determinar o tamanho desse passo, faz-se uso do método do segmento áureo [32]. Nesse método, a função objetivo tem que ser avaliada várias vezes. Isso implica que o problema primal também tem que ser resolvido para cada avaliação da função objetivo. O algoritmo do método do segmento áureo é dado a seguir.

\section{Algoritmo - Método do Segmento Áureo}

Dados um intervalo de busca $[a, b]$ do tamanho de busca do tamanho do passo $\alpha_{n}$ e uma tolerância tol, segue:

$$
\begin{aligned}
& \tau=0.5(\sqrt{5}-1) \\
& \alpha_{1}=a+(1-\tau)(b-a) \\
& \mathcal{F}_{1}=\mathcal{F}\left(\beta^{(\mathrm{n})}+\alpha_{1} s^{(\mathrm{n})}\right) \\
& \alpha_{2}=a+\tau(b-a) \\
& \mathcal{F}_{2}=\mathcal{F}\left(\beta^{(\mathrm{n})}+\alpha_{2} s^{(\mathrm{n})}\right) \\
& \text { Enquanto }(b-a)>\text { tol faça }
\end{aligned}
$$

$$
\text { Se } \mathcal{F}_{1}>\mathcal{F}_{2}
$$




$$
\begin{aligned}
& a=\alpha_{1} \\
& \alpha_{1}=\alpha_{2} \\
& \mathcal{F}_{1}=\mathcal{F}_{2} \\
& \alpha_{2}=a+\tau(b-a) \\
& \mathcal{F}_{2}=\mathcal{F}\left(\beta^{(\mathrm{n})}+\alpha_{2} s^{(\mathrm{n})}\right)
\end{aligned}
$$

Senão

$$
\begin{aligned}
& b=\alpha_{2} \\
& \alpha_{2}=\alpha_{1} \\
& \mathcal{F}_{2}=\mathcal{F}_{1} \\
& \alpha_{1}=a+(1-\tau)(b-a) \\
& \mathcal{F}_{1}=\mathcal{F}\left(\beta^{(\mathrm{n})}+\alpha_{1} s^{(\mathrm{n})}\right)
\end{aligned}
$$

fim

fim

Ao ter alcançado a tolerância tol, considere o tamanho do passo $\alpha_{n}$ como sendo o ponto médio do intervalo $[a, b]$, ou seja, $\alpha_{n}=0.5(a+b)$. Daí calcula-se $\beta^{(\mathrm{n}+1)}=\beta^{(\mathrm{n})}+\alpha_{n} s^{(\mathrm{n})}$.

- (3) Critério de parada: De posse do tamanho do passo, verifica-se se $\left\|\mathcal{F}\left(\beta^{(\mathrm{n}+1)}\right)\right\|<\mathrm{TOL}$, onde TOL é a tolerância, a qual define o critério de parada. Se esse critério for satisfeito, então pare o processo e vá para a saída (6). Caso contrário vá para o passo (5).

- (4) Cálculo dos gradientes: Com relação ao cálculo dos gradientes existem as duas seguintes possibilidades:

- Via adjunto: Com os parâmetros contidos no vetor $\beta^{(\mathrm{n}+1)}$, resolve-se o problema primal. Feito isso, faz-se a reconstrução do termo fonte do problema adjunto $\phi_{\mathrm{R}}-\phi$ e resolve-se o problema adjunto. Com as soluções do problema primal e adjunto, calcula-se as componentes do vetor gradiente $\nabla \mathcal{F}\left(\beta^{(\mathrm{n}+1)}\right)$ usando

$$
\delta_{\beta_{\mathrm{k}}} \mathcal{F}=\oint_{\partial \Omega}\left\{\delta_{\beta_{\mathrm{k}}} \kappa[\phi \nabla \psi-\psi \nabla \phi]\right\} \cdot n d s-\iint_{\Omega}\left\{\phi \nabla \cdot\left(\delta_{\beta_{\mathrm{k}}} \kappa \nabla \psi\right)\right\} d \Omega \quad \mathrm{k}=1, \ldots, \mathrm{N}
$$

Com isso, gera-se as componentes do vetor gradiente $\nabla \mathcal{F}\left(\beta^{(\mathrm{n}+1)}\right)$ e faz-se então $g=\nabla \mathcal{F}\left(\beta^{(\mathrm{n}+1)}\right)$. 
- Via diferenças finitas: Com os parâmetros contidos no vetor $\beta^{(\mathrm{n}+1)}$, resolve-se o problema primal e em seguida avalie o valor da função objetivo. Esse valor vai ser utilizado para calcular os gradientes em relação a cada parâmetro $\beta_{\mathrm{k}}$. Continuando, calcule os gradientes pelo seguinte processo

Para $\mathrm{k}=1, \ldots, \mathrm{N}$ faça

Pertube o parâmetro $\beta_{\mathrm{k}}$ e deixe os outros inalterados

Resolva o problema primal

Avalie $\mathcal{F}$ para essa perturbação

Calcule o gradiente, o qual é dado pela razão

$$
\delta_{\beta_{\mathrm{k}}} \mathcal{F}=\frac{\mathcal{F}\left[\phi\left(\beta_{\mathrm{k}}+\delta \beta_{\mathrm{k}}\right)\right]-\mathcal{F}\left[\phi\left(\beta_{\mathrm{k}}\right)\right]}{\delta \beta_{k}} \quad \mathrm{k}=1,2, \ldots, \mathrm{N}
$$

Volte o parâmetro perturbado ao valor antes da perturbação

Fim para

Com isso obtém-se as componentes do vetor gradiente $\nabla \mathcal{F}\left(\beta^{(\mathrm{n}+1)}\right)$ e faz-se $g=\nabla \mathcal{F}\left(\beta^{(\mathrm{n}+1)}\right)$.

- (5) Direção conjugada: Calcula-se o gradiente conjugado, que vai determinar a direção de pesquisa.

$$
\gamma_{\mathrm{n}+1}=\frac{\left[g^{(\mathrm{n}+1)}\right]^{\top}\left[g^{(\mathrm{n}+1)}\right]}{\left[g^{(\mathrm{n})}\right]^{\top}\left[g^{(\mathrm{n})}\right]} \Rightarrow s^{(\mathrm{n}+1)}=-g^{(\mathrm{n}+1)}+\gamma_{\mathrm{n}+1} s^{(\mathrm{n})}
$$

No para o passo (2) faz-se a busca unidirecional. O ciclo formado pelos passos (2), (3), (4) e (5) devem ser repetidos até que o critério de parada seja satisfeito.

- (6) Saída: Os parâmetros que satisfazem o critério de parada são as componentes do vetor $\beta^{(\mathrm{n}+1)}$

Os testes realizados tem por objetivo verificar a efetividade do método de obtenção dos parâmetros bem como compará-los aos métodos baseados em diferenças finitas. Os testes foram feitos em três tipos de malhas, os quais são dados na tabela 4.1

Ao aplicar o método de Runge-Kutta de multiestágio para resolver os problemas primais e duais, existe um critério de parada. O critério de parada utilizado nesse trabalho é baseado na norma 2 do resíduo, que no 


\begin{tabular}{ccc}
\hline \hline MALHA & VÉRTICES & TRIÂNGULOS \\
\hline \hline 1 & 145 & 248 \\
2 & 535 & 988 \\
3 & 2074 & 3986 \\
\hline \hline
\end{tabular}

Tabela 4.1: Parâmetros das malhas no quadrado

caso desses testes é $10^{-6}$, tanto para o primal como para o dual. O critério de parada do método de busca é baseado no valor da norma 2 do funcional. Assim sendo, a busca dos parâmetros é realizada até que a norma 2 funcional fique menor que $10^{-6}$. Outro parâmetro utilizado no método de busca é o tamanho do passo. A busca é feita até que o comprimento do intervalo $\left[\alpha_{1}, \alpha_{2}\right]$ atinja uma determinada tolerância. A tolerância utilizada é $10^{-2}$. Em todos os testes, a ordem de reconstrução utilizada é a mesma tanto para o primal como para o adjunto. Assim sendo, se o teste é feito com ordem 3, então ambos estão utilizando essa ordem de reconstrução. Se é ordem 4, o mesmo acontece.

$\mathrm{O}$ processo de busca começa com parâmetros iniciais $\beta_{\mathrm{k}}^{(0)}$ nulos para daí convergirem para $\beta_{\mathrm{k}}^{\mathrm{R}}$. Logo, a tendência da função objetivo é a convergência para zero. Os testes mostraram que a convergência ocorre mas fica limitada no sentido de que, para cada malha utilizada, existe um valor mínimo, variando entre $10^{-10}$ e $10^{-5}$, que o valor da função objetivo alcança. Essas limitações, no valor do teto mínimo alcançado, foram provocadas basicamente pelo grau de refinamento da malha e pela difusão numérica. Como será visto mais adiante com os resultados, a difusão numérica, provocada pelos erros de discretização da equação, atrapalha a busca dos parâmetros que determinam a difusão física, que existe na equação de advecção-difusão, e precisa ser determinada.

Um dos objetivos desse trabalho reside justamente em estudar o efeito da técnica de reconstrução de alta ordem ao empregar a técnica do operador adjunto no cálculo dos gradientes, quando comparado com os gradientes calculados via técnica de diferenças finitas. Como a difusão numérica tem efeito nessas duas estratégias, um outro objetivo reside em estudar sobre qual das referidas estratégias a difusão numérica tem menos efeito. Em outras palavras, isso significa estudar qual das duas técnicas de cálculo de gradientes, usando reconstrução de alta ordem, consegue separar a difusão numérica da difusão física. Separar cem por cento não é possível, mas o que se deseja é identificar qual estratégia consegue estimar com um bom nível de precisão os parâmetros que determinam o coeficiente de difusão. 
Todos os testes foram realizados no Cluster Beowulf Alcatéia, constituido de um nó servidor e 32 nós clientes conectados via Ethernet, baseados em CPUs Intel Dual Xeon de $2.8 \mathrm{GHz}$ e 2.5GB de memória RAM, equipado com discos SCSI com 72GB para armazenamento dos usuários e aritmética de 32 bits e o cluster está localizado no Laboratório de Computação Científica Avançada (LCCA) do Centro de Computação Eletrônica (CCE) da Universidade de São Paulo.

As malhas utilizadas foram geradas utilizando-se o emc2, um gerador de malhas bidimensional, desenvolvido pelo INRIA na França por Eric e Hecht [54].

As próximas seções apresentam os testes realizados bem como os resultados com seus respectivos comentários.

\subsection{Problema teste 1: estimação de dois parâmetros - função linear}

Esse problema teste consiste em estimar os parâmetros $a$ e $b$ do coeficiente de difusão,

$$
\kappa(x, y)=a x+b y+C_{0}
$$

onde $C_{0}=0.05$, presente na equação de advecção-difusão

$$
\nabla \cdot(\vec{\omega} \phi)-\nabla \cdot(\kappa \nabla \phi)=0 \quad(x, y) \in \Omega \subset \mathbb{R}^{2}
$$

definida no quadrado unitário $\Omega=[0,1] \times[0,1]$, sendo $\vec{\omega}=(1,0)$, e tendo como condições de fronteira

$$
\begin{aligned}
& \phi(0, y)=\left\{\begin{array}{ll}
1, & 0 \leq y \leq 0.4 \\
5 y-1, & 0.4<y<0.6 \\
2, & 0.6 \leq y \leq 1.0
\end{array} \quad \phi(x, 0)=1 \quad 0<x \leq 1\right. \\
& \phi(x, 1)=2 \quad 0<x \leq 1 \quad \frac{\partial \phi(1, y)}{\partial n}=0 \quad 0<y<1
\end{aligned}
$$

utilizando como ferramenta um problema otimização que consiste em minimizar o funcional

$$
\mathcal{F}[\kappa]=\frac{1}{2} \iint_{\Omega}\left(\phi-\phi_{\mathrm{R}}\right)^{2} d \Omega
$$

tendo como restrição a equação de advecção-difusão apresentada.

Perceba que essa função de teste foi apresentada no capítulo 2, no qual a equação de advecção com condições de fronteira pela função teste foi resolvida em três tipos de malhas. No referido capítulo, na última 
seção, foram apresentados dois gráficos mostrando que o refinamento da malha ameniza os efeitos difusão numérica, os quais são provocados pelos erros de discretização, no caso, da equação de advecção.

Continuando, o problema adjunto é dado por

$$
-\nabla \cdot(\vec{\omega} \psi)-\nabla \cdot(\kappa \nabla \psi)=0 \quad(x, y) \in \Omega \subset \mathbb{R}^{2}
$$

definida no quadrado unitário $\Omega=[0,1] \times[0,1]$, sendo $\vec{\omega}=(1,0)$, e tendo como condições de fronteira

$$
\begin{gathered}
\psi(0, y)=\left\{\begin{array}{ll}
0, & 0 \leq y \leq 0.4 \\
0, & 0.4<y<0.6 \\
0, & 0.6 \leq y \leq 1.0
\end{array} \quad \psi(x, 0)=0 \quad 0<x \leq 1\right. \\
\psi(x, 1)=0 \quad 0<x \leq 1 \quad[\vec{\omega} \psi+\kappa \nabla \psi] \cdot n=0 \quad 0<y<1
\end{gathered}
$$

Para esse teste, foi gerado um $\phi^{\mathrm{R}}$ de referência utilizando $a_{\mathrm{R}}=b_{\mathrm{R}}=0.1$. O processo de busca foi inicializado tomando como parâmetros iniciais $a=b=0$. Assim sendo, o processo de busca deverá levar os parâmetros iniciais para $a_{\mathrm{R}}=b_{\mathrm{R}}=0.1$. Para comparação, a estimativa de parâmetros também foi feita calculando os gradientes usando diferenças finitas, sendo $\delta \beta_{\mathrm{k}}=0.01$ a perturbação (ou tamanho do passo). Assim sendo, no caso, as perturbações utilizadas para calcular os gradientes via diferenças finitas foram $\delta a=\delta b=0.01$.

A tabela 4.2 apresenta uma descrição das colunas das tabelas de resultados. Nas tabelas de resultados, MGC é abreviação de Método dos Gradientes Conjugados e MRAO de Método de Reconstrução de Alta Ordem.

\begin{tabular}{|c|c|l|}
\hline $1^{a}$ & it & iterações \\
\hline $2^{a}$ & $\mathcal{F}$ & valor do funcional \\
\hline $3^{a}$ & $\delta_{a} \mathcal{F}$ & gradiente em relação a $a$ \\
\hline $4^{a}$ & $\delta_{b} \mathcal{F}$ & gradiente em relação a $b$ \\
\hline $5^{a}$ & $\|\delta \mathcal{F}\|$ & norma 2 do gradiente \\
\hline $7^{a}$ & $\mathrm{CP}$ & número de chamadas do primal \\
\hline $8^{a}$ & $\mathrm{t}(\mathrm{seg})$ & tempo gasto na busca unidirecional (em segundos) \\
\hline $9^{a}$ & $\mathrm{TCG}$ & tempo de cálculo do gradiente (em segundos) \\
\hline $10^{a}$ & $\mathrm{a}$ & estimativa do primeiro parâmetro \\
\hline $11^{a}$ & $\mathrm{~b}$ & estimativa do segundo parâmetro \\
\hline
\end{tabular}

Tabela 4.2: Teste 1: Descrição das tabelas

As figuras 4.3 e 4.4 mostram, respectivamente, os gráficos da solução de referência $\left(\right.$ com $\left.a_{\mathrm{R}}=b_{\mathrm{R}}=0.1\right)$ 
e o gráfico do coeficiente de difusão com os parâmetros de referência. Note que a variação da função $\kappa(x, y)$ é linear e seus valores variam de 0.05 até 0.25 . Essa variação provoca no gráfico 4.6 a abertura em forma de leque, um espalhamento, característica de fenômenos difusivos. A figura 4.5 mostra a solução $\phi$ inicial, onde $k(x, y)=0.05$ em todo domínio. Comparando $4.6 \mathrm{com} 4.5$ pode-se ver que os valores em valor absoluto são pequenos. As figuras 4.6 e 4.7 apresentam, respectivamente, os gráficos dos termos fontes do adjunto inicial e a solução do problema adjunto inicial. Note que os valores do termo fonte são pequenos, assim como da solução do problema adjunto inicial. Esses valores tendem a diminuir cada vez mais, pois a medida que os parâmetros se aproximam dos parâmetros ótimos, a solução do problema adjunto tende a zero, uma vez que o termo fonte do problema adjunto é dado por $\phi-\phi_{\mathrm{R}}$.

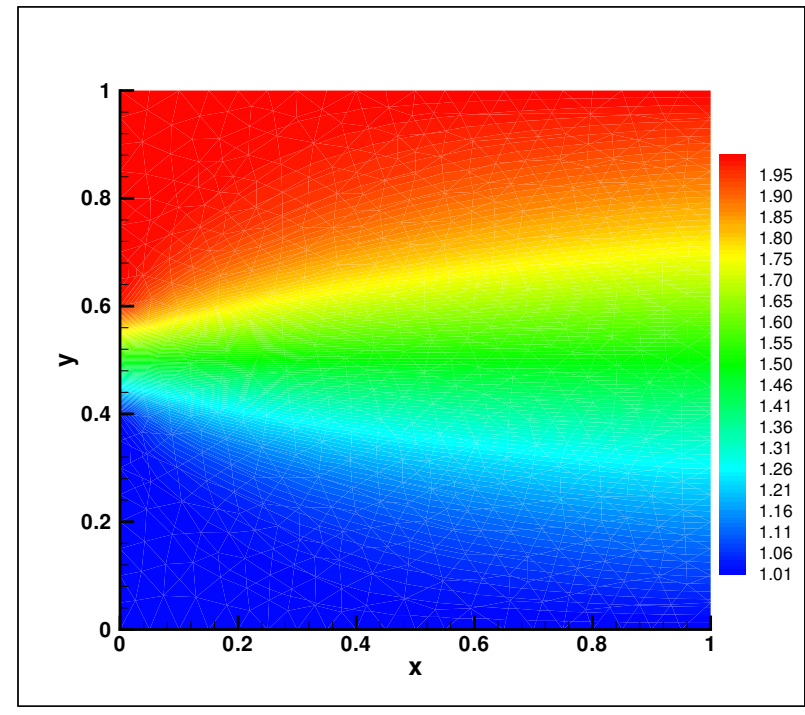

Figura 4.3: Solução de referência $\phi_{R}$

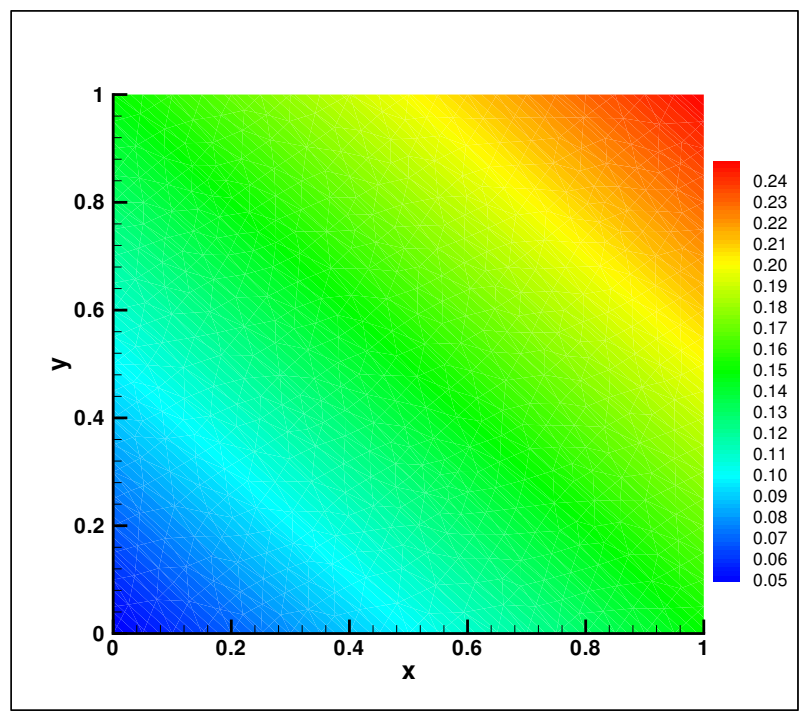

Figura 4.4: Gráfico de $k(x, y)=0.1 x+0.1 y+0.05$

Uma observação deve ser feita aqui. Perceba que o problema dual tem 3 fronteiras sob condição de Dirichlet onde $\psi=0$, e uma fronteira sob condição de Robin $[\vec{\omega} \psi+\kappa \nabla \psi] \cdot n=0$. A medida que o vetor dos parâmetros $(a, b)$ tende a $\left(a_{\mathrm{R}}, b_{\mathrm{R}}\right)$, o termo fonte $\phi_{\mathrm{R}}-\phi$ tende a zero. Logo a solução do problema adjunto tende a zero em todo o domínio, uma vez que a solução da fronteira nula $(\psi=0)$ tende a se propagar por todo o domínio. Note ainda que na equação adjunta, quando o termo fonte tende a zero, independente do quanto $\kappa$ esteja impreciso, a solução tende a zero. No caso dos gradientes calculados via diferenças finitas, nas proximidades de $\left(a_{\mathrm{R}}, b_{\mathrm{R}}\right)$ ocorre muita pouca variação, quase nula, nas soluções $\phi$ do problema primal ao fazer as perturbações nos parâmetros $\beta_{k}$ para calcular os gradientes. Com isso, a razão em (4.3) é muito pequena. Isso dificulta obter um vetor gradiente que proporcione avanços na direção dos parâmetros $\left(a_{\mathrm{R}}, b_{\mathrm{R}}\right)$. 


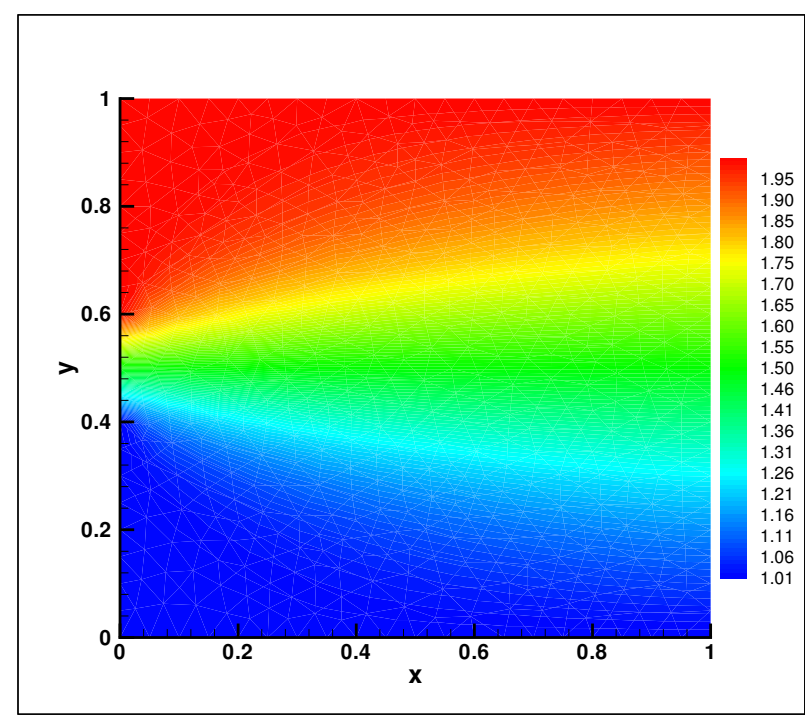

Figura 4.5: Gráfico de $\phi$ inicial

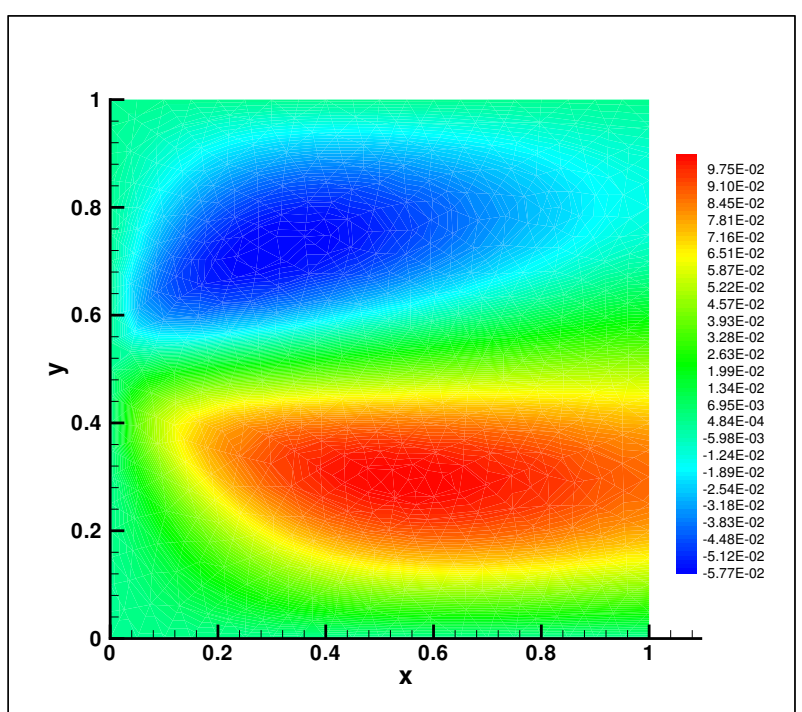

Figura 4.6: Termo fonte do adjunto inicial $\phi_{R}-\phi$

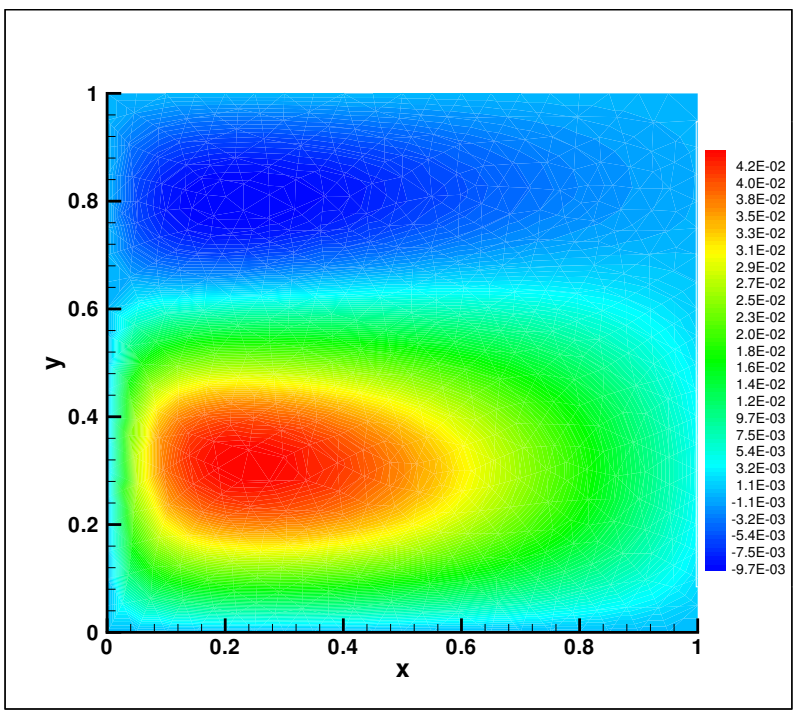

Figura 4.7: Gráfico da solução adjunta inicial $\psi$ 
Assim sendo, tanto o gradiente calculado via método adjunto como via diferenças finitas tem seus problemas, e um dos objetivos dos testes consistem em verificar qual das duas abordagens consegue capturar melhor os gradientes.

As tabelas 4.3 e 4.4 apresentam, respectivamente, os resultados obtidos, usando recontrução de ordem 3 na malha 1, calculando os gradientes via método adjunto e via diferenças finitas. O gráfico 4.8 apresenta a evolução dos parâmetros referentes a forma funcional da difusão.

\begin{tabular}{cccccccccc}
\hline \hline it & $\mathcal{F}$ & $\delta_{a} \mathcal{F}$ & $\delta_{b} \mathcal{F}$ & $\|\delta \mathcal{F}\|$ & CP & t(seg) & TCG & a & b \\
\hline \hline 0 & $1.3 \mathrm{e}-03$ & $2.0 \mathrm{e}-02$ & $6.2 \mathrm{e}-02$ & $6.5 \mathrm{e}-02$ & 1 & 24 & 41 & 0.000 & 0.000 \\
1 & $2.2 \mathrm{e}-05$ & $1.0 \mathrm{e}-03$ & $-4.6 \mathrm{e}-04$ & $1.1 \mathrm{e}-03$ & 13 & 161 & 28 & 0.035 & 0.109 \\
2 & $7.5 \mathrm{e}-06$ & $2.8 \mathrm{e}-03$ & $5.8 \mathrm{e}-04$ & $2.8 \mathrm{e}-03$ & 21 & 217 & 23 & 0.071 & 0.093 \\
3 & $1.2 \mathrm{e}-08$ & $2.0 \mathrm{e}-05$ & $1.7 \mathrm{e}-04$ & $1.7 \mathrm{e}-04$ & 19 & 169 & 25 & 0.101 & 0.100 \\
\hline \hline
\end{tabular}

Tabela 4.3: Malha 1: MGC com MRAO de ordem 3 usando método adjunto

\begin{tabular}{cccccccccc}
\hline \hline it & $\mathcal{F}$ & $\delta_{a} \mathcal{F}$ & $\delta_{b} \mathcal{F}$ & $\|\delta \mathcal{F}\|$ & CP & t(seg) & TCG & a & b \\
\hline \hline 0 & $1.4 \mathrm{e}-03$ & $1.6 \mathrm{e}-02$ & $2.8 \mathrm{e}-02$ & $3.2 \mathrm{e}-02$ & 1 & 24 & 53 & 0.000 & 0.000 \\
1 & $7.5 \mathrm{e}-06$ & $3.1 \mathrm{e}-04$ & $-6.6 \mathrm{e}-04$ & $7.3 \mathrm{e}-04$ & 14 & 193 & 34 & 0.061 & 0.107 \\
2 & $6.4 \mathrm{e}-06$ & $4.2 \mathrm{e}-04$ & $-4.8 \mathrm{e}-04$ & $6.4 \mathrm{e}-04$ & 21 & 239 & 39 & 0.063 & 0.102 \\
3 & $6.3 \mathrm{e}-06$ & $6.1 \mathrm{e}-04$ & $-4.8 \mathrm{e}-04$ & $7.7 \mathrm{e}-04$ & 22 & 254 & 31 & 0.064 & 0.100 \\
4 & $6.1 \mathrm{e}-06$ & $7.7 \mathrm{e}-04$ & $-3.7 \mathrm{e}-04$ & $8.5 \mathrm{e}-04$ & 21 & 242 & 41 & 0.067 & 0.098 \\
5 & $5.6 \mathrm{e}-06$ & $1.1 \mathrm{e}-03$ & $-2.3 \mathrm{e}-04$ & $1.2 \mathrm{e}-03$ & 21 & 236 & 42 & 0.072 & 0.095 \\
6 & $3.7 \mathrm{e}-06$ & $1.4 \mathrm{e}-03$ & $6.9 \mathrm{e}-05$ & $1.4 \mathrm{e}-03$ & 20 & 195 & 49 & 0.091 & 0.092 \\
7 & $2.1 \mathrm{e}-06$ & $2.6 \mathrm{e}-04$ & $1.7 \mathrm{e}-04$ & $3.1 \mathrm{e}-04$ & 20 & 188 & 46 & 0.108 & 0.092 \\
8 & $1.1 \mathrm{e}-06$ & $3.1 \mathrm{e}-04$ & $1.2 \mathrm{e}-04$ & $3.3 \mathrm{e}-04$ & 23 & 273 & 35 & 0.115 & 0.097 \\
9 & $1.1 \mathrm{e}-06$ & $1.8 \mathrm{e}-04$ & $-5.4 \mathrm{e}-05$ & $1.8 \mathrm{e}-04$ & 22 & 225 & 42 & 0.115 & 0.097 \\
10 & $1.1 \mathrm{e}-06$ & $4.1 \mathrm{e}-05$ & $-2.3 \mathrm{e}-04$ & $2.3 \mathrm{e}-04$ & 24 & 282 & 29 & 0.115 & 0.097 \\
11 & $1.1 \mathrm{e}-06$ & $-9.6 \mathrm{e}-05$ & $-4.0 \mathrm{e}-04$ & $4.1 \mathrm{e}-04$ & 23 & 261 & 33 & 0.115 & 0.097 \\
12 & $1.1 \mathrm{e}-06$ & $-2.3 \mathrm{e}-04$ & $-5.9 \mathrm{e}-04$ & $6.3 \mathrm{e}-04$ & 22 & 247 & 32 & 0.115 & 0.097 \\
13 & $1.1 \mathrm{e}-06$ & $-3.7 \mathrm{e}-04$ & $-7.8 \mathrm{e}-04$ & $8.6 \mathrm{e}-04$ & 21 & 270 & 53 & 0.115 & 0.097 \\
14 & $1.1 \mathrm{e}-06$ & $-4.9 \mathrm{e}-04$ & $-9.1 \mathrm{e}-04$ & $1.0 \mathrm{e}-03$ & 21 & 247 & 34 & 0.115 & 0.097 \\
15 & $1.1 \mathrm{e}-06$ & $-6.5 \mathrm{e}-04$ & $-1.1 \mathrm{e}-03$ & $1.3 \mathrm{e}-03$ & 20 & 214 & 47 & 0.115 & 0.097 \\
16 & $1.1 \mathrm{e}-06$ & $-7.9 \mathrm{e}-04$ & $-1.3 \mathrm{e}-03$ & $1.5 \mathrm{e}-03$ & 20 & 239 & 45 & 0.115 & 0.097 \\
17 & $1.1 \mathrm{e}-06$ & $-8.8 \mathrm{e}-04$ & $-1.4 \mathrm{e}-03$ & $1.7 \mathrm{e}-03$ & 20 & 238 & 39 & 0.115 & 0.097 \\
18 & $1.1 \mathrm{e}-06$ & $-1.0 \mathrm{e}-03$ & $-1.6 \mathrm{e}-03$ & $1.9 \mathrm{e}-03$ & 19 & 239 & 49 & 0.115 & 0.097 \\
19 & $1.1 \mathrm{e}-06$ & $-1.2 \mathrm{e}-03$ & $-1.8 \mathrm{e}-03$ & $2.2 \mathrm{e}-03$ & 19 & 248 & 43 & 0.115 & 0.097 \\
20 & $1.1 \mathrm{e}-06$ & $-1.3 \mathrm{e}-03$ & $-1.9 \mathrm{e}-03$ & $2.3 \mathrm{e}-03$ & 19 & 236 & 31 & 0.115 & 0.097 \\
\hline \hline
\end{tabular}

Tabela 4.4: Malha 1: MGC com MRAO de ordem 3 usando diferenças finitas

O método adjunto na primeira iteração não chega tão perto da solução quanto diferenças finitas, porém 
em pouca iterações atinge o critério de parada e os parâmetros estimados tem precisão superior ao calculado via diferenças finitas. Note que diferenças finitas logo na primeira iteração já chegou perto da convergência. Entretanto levou mais 20 iterações e não atingiu o critério de convergência. Isso se deve ao fato da fórmula de primeira ordem utilizado para o cálculo do gradiente envolver o cálculo de dois problemas primais com coeficientes de difusão diferindo de 0.01. Essa razão de valores pequenos aparentemente não é precisa o suficiente para o cálculo correto do gradiente. Percebe-se que apesar de afetados pelo erro numérico, ambos os métodos se aproximam da solução do problema inverso de teste. Entretanto, na proximidade da solução o cálculo do gradiente por diferenças finitas não tem a precisão necessária para atingir o critério de convergência.

As tabelas 4.5 e 4.6 apresentam, respectivamente, os resultados obtidos, usando recontrução de ordem 3 na malha 2, calculando os gradientes via método adjunto e via diferenças finitas. O gráfico 4.10 apresenta a evolução dos parâmetros. Os resultados mostram que, essencialmente, houve o mesmo comportamento da malha 1 , com o crescimento no tempo computacional decorrente do maior número de vértices. Note que novamente o método de diferenças finitas não alcançou a precisão especificada.

\begin{tabular}{cccccccccc}
\hline \hline it & $\mathcal{F}$ & $\delta_{a} \mathcal{F}$ & $\delta_{b} \mathcal{F}$ & $\|\delta \mathcal{F}\|$ & CP & t(seg) & TCG & a & b \\
\hline \hline 0 & $1.3 \mathrm{e}-03$ & $1.9 \mathrm{e}-02$ & $4.8 \mathrm{e}-02$ & $5.1 \mathrm{e}-02$ & 1 & 89 & 148 & 0.000 & 0.000 \\
1 & $1.6 \mathrm{e}-05$ & $7.2 \mathrm{e}-04$ & $-5.6 \mathrm{e}-04$ & $9.2 \mathrm{e}-04$ & 13 & 454 & 53 & 0.044 & 0.109 \\
2 & $9.7 \mathrm{e}-06$ & $1.7 \mathrm{e}-03$ & $1.3 \mathrm{e}-04$ & $1.7 \mathrm{e}-03$ & 21 & 464 & 73 & 0.059 & 0.097 \\
3 & $6.4 \mathrm{e}-09$ & $3.0 \mathrm{e}-05$ & $-9.3 \mathrm{e}-06$ & $3.2 \mathrm{e}-05$ & 20 & 325 & 30 & 0.100 & 0.100 \\
\hline \hline
\end{tabular}

Tabela 4.5: Malha 2: MGC com MRAO de ordem 3 usando método adjunto

As tabelas 4.7 e 4.8 apresentam, respectivamente, os resultados obtidos, usando recontrução de ordem 3 na malha 3, calculando os gradientes via método adjunto e via diferenças finitas. O gráfico 4.12 apresenta a evolução dos parâmetros. Nesse teste, apenas ocorre o aumento do custo computacional devido ao aumento do número de vértices mantendo as tendências dos casos anteriores.

Nas três malhas, utilizando reconstrução de ordem 3, o método adjunto conseguiu atingir a precisão especificada, enquanto que o método de diferenças finitas não. Os resultados indicam que, mesmo com a difusão numérica envolvida no processo, o método adjunto consegue capturar os gradientes com precisão o suficiente para caminhar em direção aos parâmetros ótimos.

Considere agora os testes com ordem mais alta, no caso ordem 4. As tabelas 4.9 e 4.10 apresentam, respectivamente, os resultados obtidos, usando recontrução de ordem 4 na malha 3, calculando os gradientes via método adjunto e via diferenças finitas, sendo que o gráfico 4.9 apresenta a evolução dos parâmetros. No 


\begin{tabular}{cccccccccc}
\hline \hline it & $\mathcal{F}$ & $\delta_{a} \mathcal{F}$ & $\delta_{b} \mathcal{F}$ & $\|\delta \mathcal{F}\|$ & $\mathrm{CP}$ & $\mathrm{t}(\mathrm{seg})$ & $\mathrm{TCG}$ & $\mathrm{a}$ & $\mathrm{b}$ \\
\hline \hline 0 & $1.3 \mathrm{e}-03$ & $1.6 \mathrm{e}-02$ & $2.8 \mathrm{e}-02$ & $3.3 \mathrm{e}-02$ & 1 & 162 & 402 & 0.000 & 0.000 \\
1 & $7.5 \mathrm{e}-06$ & $3.1 \mathrm{e}-04$ & $-6.5 \mathrm{e}-04$ & $7.2 \mathrm{e}-04$ & 14 & 547 & 85 & 0.061 & 0.107 \\
2 & $6.2 \mathrm{e}-06$ & $4.1 \mathrm{e}-04$ & $-4.9 \mathrm{e}-04$ & $6.4 \mathrm{e}-04$ & 21 & 609 & 176 & 0.064 & 0.102 \\
3 & $5.9 \mathrm{e}-06$ & $5.4 \mathrm{e}-04$ & $-3.6 \mathrm{e}-04$ & $6.5 \mathrm{e}-04$ & 22 & 744 & 164 & 0.066 & 0.099 \\
4 & $5.7 \mathrm{e}-06$ & $8.1 \mathrm{e}-04$ & $-2.1 \mathrm{e}-04$ & $8.4 \mathrm{e}-04$ & 22 & 583 & 160 & 0.071 & 0.096 \\
5 & $4.2 \mathrm{e}-06$ & $1.4 \mathrm{e}-03$ & $4.8 \mathrm{e}-05$ & $1.4 \mathrm{e}-03$ & 21 & 498 & 297 & 0.089 & 0.091 \\
6 & $2.4 \mathrm{e}-06$ & $2.3 \mathrm{e}-04$ & $1.8 \mathrm{e}-04$ & $2.9 \mathrm{e}-04$ & 20 & 411 & 170 & 0.111 & 0.092 \\
7 & $1.2 \mathrm{e}-06$ & $2.0 \mathrm{e}-04$ & $9.2 \mathrm{e}-05$ & $2.2 \mathrm{e}-04$ & 23 & 530 & 75 & 0.117 & 0.097 \\
8 & $1.2 \mathrm{e}-06$ & $1.0 \mathrm{e}-04$ & $-9.2 \mathrm{e}-05$ & $1.4 \mathrm{e}-04$ & 23 & 442 & 122 & 0.118 & 0.097 \\
9 & $1.2 \mathrm{e}-06$ & $-6.0 \mathrm{e}-05$ & $-2.8 \mathrm{e}-04$ & $2.8 \mathrm{e}-04$ & 24 & 471 & 89 & 0.118 & 0.097 \\
10 & $1.2 \mathrm{e}-06$ & $-2.1 \mathrm{e}-04$ & $-4.7 \mathrm{e}-04$ & $5.2 \mathrm{e}-04$ & 23 & 591 & 73 & 0.118 & 0.097 \\
11 & $1.2 \mathrm{e}-06$ & $-3.9 \mathrm{e}-04$ & $-7.3 \mathrm{e}-04$ & $8.2 \mathrm{e}-04$ & 22 & 524 & 197 & 0.118 & 0.097 \\
12 & $1.2 \mathrm{e}-06$ & $-5.0 \mathrm{e}-04$ & $-8.6 \mathrm{e}-04$ & $9.9 \mathrm{e}-04$ & 21 & 475 & 77 & 0.118 & 0.097 \\
13 & $1.2 \mathrm{e}-06$ & $-6.5 \mathrm{e}-04$ & $-1.0 \mathrm{e}-03$ & $1.2 \mathrm{e}-03$ & 20 & 501 & 88 & 0.118 & 0.097 \\
14 & $1.2 \mathrm{e}-06$ & $-8.0 \mathrm{e}-04$ & $-1.2 \mathrm{e}-03$ & $1.5 \mathrm{e}-03$ & 20 & 466 & 96 & 0.118 & 0.097 \\
15 & $1.2 \mathrm{e}-06$ & $-1.0 \mathrm{e}-03$ & $-1.6 \mathrm{e}-03$ & $1.9 \mathrm{e}-03$ & 20 & 665 & 129 & 0.118 & 0.097 \\
16 & $1.2 \mathrm{e}-06$ & $-1.2 \mathrm{e}-03$ & $-1.8 \mathrm{e}-03$ & $2.1 \mathrm{e}-03$ & 19 & 503 & 151 & 0.118 & 0.097 \\
17 & $1.2 \mathrm{e}-06$ & $-1.2 \mathrm{e}-03$ & $-1.8 \mathrm{e}-03$ & $2.2 \mathrm{e}-03$ & 19 & 462 & 90 & 0.118 & 0.097 \\
18 & $1.2 \mathrm{e}-06$ & $-1.4 \mathrm{e}-03$ & $-2.0 \mathrm{e}-03$ & $2.4 \mathrm{e}-03$ & 19 & 684 & 73 & 0.118 & 0.097 \\
19 & $1.2 \mathrm{e}-06$ & $-1.5 \mathrm{e}-03$ & $-2.2 \mathrm{e}-03$ & $2.7 \mathrm{e}-03$ & 19 & 461 & 83 & 0.118 & 0.097 \\
20 & $1.2 \mathrm{e}-06$ & $-1.7 \mathrm{e}-03$ & $-2.3 \mathrm{e}-03$ & $2.9 \mathrm{e}-03$ & 18 & 483 & 73 & 0.118 & 0.097 \\
\hline \hline
\end{tabular}

Tabela 4.6: Malha 2: MGC com MRAO de ordem 3 usando diferenças finitas

\begin{tabular}{cccccccccc}
\hline \hline it & $\mathcal{F}$ & $\delta_{a} \mathcal{F}$ & $\delta_{b} \mathcal{F}$ & $\|\delta \mathcal{F}\|$ & CP & t(seg) & TCG & a & b \\
\hline \hline 0 & $1.3 \mathrm{e}-03$ & $1.8 \mathrm{e}-02$ & $4.4 \mathrm{e}-02$ & $4.8 \mathrm{e}-02$ & 1 & 277 & 459 & 0.000 & 0.000 \\
1 & $1.6 \mathrm{e}-05$ & $6.8 \mathrm{e}-04$ & $-3.9 \mathrm{e}-04$ & $7.9 \mathrm{e}-04$ & 14 & 1241 & 137 & 0.045 & 0.109 \\
2 & $6.8 \mathrm{e}-06$ & $2.1 \mathrm{e}-03$ & $1.9 \mathrm{e}-04$ & $2.1 \mathrm{e}-03$ & 21 & 956 & 22 & 0.072 & 0.094 \\
3 & $3.4 \mathrm{e}-07$ & $2.0 \mathrm{e}-04$ & $4.2 \mathrm{e}-04$ & $4.7 \mathrm{e}-04$ & 19 & 911 & 32 & 0.106 & 0.097 \\
\hline \hline
\end{tabular}

Tabela 4.7: Malha 3: MGC com MRAO de ordem 3 usando método adjunto 


\begin{tabular}{cccccccccc}
\hline \hline it & $\mathcal{F}$ & $\delta_{a} \mathcal{F}$ & $\delta_{b} \mathcal{F}$ & $\|\delta \mathcal{F}\|$ & $C P$ & $\mathrm{t}(\mathrm{seg})$ & $\mathrm{TCG}$ & $\mathrm{a}$ & $\mathrm{b}$ \\
\hline \hline 0 & $1.3 \mathrm{e}-03$ & $1.6 \mathrm{e}-02$ & $2.8 \mathrm{e}-02$ & $3.3 \mathrm{e}-02$ & 1 & 321 & 862 & 0.000 & 0.000 \\
1 & $7.7 \mathrm{e}-06$ & $3.2 \mathrm{e}-04$ & $-6.2 \mathrm{e}-04$ & $6.9 \mathrm{e}-04$ & 14 & 1208 & 448 & 0.062 & 0.107 \\
2 & $6.3 \mathrm{e}-06$ & $4.2 \mathrm{e}-04$ & $-4.7 \mathrm{e}-04$ & $6.3 \mathrm{e}-04$ & 21 & 1286 & 323 & 0.064 & 0.102 \\
3 & $5.6 \mathrm{e}-06$ & $4.9 \mathrm{e}-04$ & $-3.5 \mathrm{e}-04$ & $6.0 \mathrm{e}-04$ & 22 & 1168 & 482 & 0.067 & 0.099 \\
4 & $5.6 \mathrm{e}-06$ & $7.9 \mathrm{e}-04$ & $-2.9 \mathrm{e}-04$ & $8.4 \mathrm{e}-04$ & 22 & 1038 & 244 & 0.069 & 0.097 \\
5 & $4.6 \mathrm{e}-06$ & $1.3 \mathrm{e}-03$ & $-1.1 \mathrm{e}-04$ & $1.3 \mathrm{e}-03$ & 21 & 924 & 407 & 0.080 & 0.093 \\
6 & $2.6 \mathrm{e}-06$ & $7.2 \mathrm{e}-04$ & $2.0 \mathrm{e}-04$ & $7.4 \mathrm{e}-04$ & 20 & 818 & 245 & 0.100 & 0.091 \\
7 & $1.1 \mathrm{e}-06$ & $4.0 \mathrm{e}-05$ & $-6.8 \mathrm{e}-06$ & $4.0 \mathrm{e}-05$ & 21 & 982 & 335 & 0.113 & 0.095 \\
8 & $1.1 \mathrm{e}-06$ & $-6.7 \mathrm{e}-05$ & $-5.4 \mathrm{e}-05$ & $8.6 \mathrm{e}-05$ & 27 & 1020 & 327 & 0.113 & 0.095 \\
9 & $1.1 \mathrm{e}-06$ & $-1.7 \mathrm{e}-04$ & $-1.0 \mathrm{e}-04$ & $2.0 \mathrm{e}-04$ & 26 & 1195 & 403 & 0.113 & 0.095 \\
10 & $1.1 \mathrm{e}-06$ & $-2.8 \mathrm{e}-04$ & $-1.5 \mathrm{e}-04$ & $3.2 \mathrm{e}-04$ & 24 & 910 & 247 & 0.113 & 0.095 \\
11 & $1.1 \mathrm{e}-06$ & $-3.9 \mathrm{e}-04$ & $-1.9 \mathrm{e}-04$ & $4.3 \mathrm{e}-04$ & 23 & 1008 & 224 & 0.113 & 0.095 \\
12 & $1.1 \mathrm{e}-06$ & $-4.9 \mathrm{e}-04$ & $-2.4 \mathrm{e}-04$ & $5.5 \mathrm{e}-04$ & 22 & 889 & 333 & 0.113 & 0.095 \\
13 & $1.1 \mathrm{e}-06$ & $-6.0 \mathrm{e}-04$ & $-2.9 \mathrm{e}-04$ & $6.6 \mathrm{e}-04$ & 22 & 1007 & 278 & 0.113 & 0.095 \\
14 & $1.1 \mathrm{e}-06$ & $-7.0 \mathrm{e}-04$ & $-3.3 \mathrm{e}-04$ & $7.7 \mathrm{e}-04$ & 21 & 859 & 155 & 0.113 & 0.095 \\
15 & $1.1 \mathrm{e}-06$ & $-8.1 \mathrm{e}-04$ & $-3.8 \mathrm{e}-04$ & $8.9 \mathrm{e}-04$ & 21 & 1127 & 175 & 0.113 & 0.095 \\
16 & $1.1 \mathrm{e}-06$ & $-9.2 \mathrm{e}-04$ & $-4.2 \mathrm{e}-04$ & $1.0 \mathrm{e}-03$ & 21 & 970 & 207 & 0.113 & 0.095 \\
17 & $1.1 \mathrm{e}-06$ & $-1.0 \mathrm{e}-03$ & $-4.7 \mathrm{e}-04$ & $1.1 \mathrm{e}-03$ & 20 & 888 & 186 & 0.113 & 0.095 \\
18 & $1.1 \mathrm{e}-06$ & $-1.1 \mathrm{e}-03$ & $-5.1 \mathrm{e}-04$ & $1.2 \mathrm{e}-03$ & 20 & 795 & 199 & 0.113 & 0.095 \\
19 & $1.1 \mathrm{e}-06$ & $-1.2 \mathrm{e}-03$ & $-5.6 \mathrm{e}-04$ & $1.4 \mathrm{e}-03$ & 20 & 915 & 181 & 0.113 & 0.095 \\
20 & $1.1 \mathrm{e}-06$ & $-1.3 \mathrm{e}-03$ & $-6.0 \mathrm{e}-04$ & $1.5 \mathrm{e}-03$ & 20 & 847 & 147 & 0.113 & 0.095 \\
\hline \hline
\end{tabular}

Tabela 4.8: Malha 3: MGC com MRAO de ordem 3 usando diferenças finitas 
caso adjunto, em uma única iteração a precisão do funcional foi atingida. Entretando o custo computacional foi maior por se utilizar quarta ordem (cerca de 50\%). Para essa ordem o cálculo do gradiente por diferenças finitas permitiu atingir a ordem de convergência do funcional em 7 iterações, entretanto a um custo cerca de 8 vezes maior se comparar com o método adjunto.

Se compararmos o gráfico 4.8 com o gráfico 4.9 , percebe-se que:

- O aumento de ordem fez com que o primeiro cálculo do gradiente fosse mais preciso, levando a convergência num único passo.

- O aumento de ordem permitiu a convergência do método de diferenças finitas, entretanto ainda exibindo dificuldade de convergência na proximidade da solução.

- Na figura 4.8 percebe-se que mesmo partindo de um gradiente inicial menos preciso, o método adjunto foi capaz de convergir para a solução na precisão especificada.

\begin{tabular}{cccccccccc}
\hline \hline it & $\mathcal{F}$ & $\delta_{a} \mathcal{F}$ & $\delta_{b} \mathcal{F}$ & $\|\delta \mathcal{F}\|$ & CP & t(seg) & TCG & a & b \\
\hline \hline 0 & $1.3 \mathrm{e}-03$ & $1.1 \mathrm{e}-02$ & $9.8 \mathrm{e}-03$ & $1.5 \mathrm{e}-02$ & 1 & 97 & 168 & 0.000 & 0.000 \\
1 & $4.8 \mathrm{e}-07$ & $6.6 \mathrm{e}-06$ & $2.8 \mathrm{e}-04$ & $2.8 \mathrm{e}-04$ & 16 & 685 & 79 & 0.110 & 0.098 \\
\hline \hline
\end{tabular}

Tabela 4.9: Malha 1: MGC com MRAO de ordem 4 usando método adjunto

\begin{tabular}{cccccccccc}
\hline \hline it & $\mathcal{F}$ & $\delta_{a} \mathcal{F}$ & $\delta_{b} \mathcal{F}$ & $\|\delta \mathcal{F}\|$ & CP & t(seg) & TCG & a & b \\
\hline \hline 0 & $1.3 \mathrm{e}-03$ & $1.5 \mathrm{e}-02$ & $2.7 \mathrm{e}-02$ & $3.1 \mathrm{e}-02$ & 1 & 115 & 262 & 0.000 & 0.000 \\
1 & $7.0 \mathrm{e}-06$ & $3.0 \mathrm{e}-04$ & $-5.9 \mathrm{e}-04$ & $6.6 \mathrm{e}-04$ & 15 & 772 & 159 & 0.061 & 0.106 \\
2 & $6.1 \mathrm{e}-06$ & $4.0 \mathrm{e}-04$ & $-4.1 \mathrm{e}-04$ & $5.7 \mathrm{e}-04$ & 21 & 1071 & 163 & 0.064 & 0.101 \\
3 & $5.9 \mathrm{e}-06$ & $5.7 \mathrm{e}-04$ & $-3.6 \mathrm{e}-04$ & $6.7 \mathrm{e}-04$ & 22 & 1083 & 210 & 0.066 & 0.099 \\
4 & $5.6 \mathrm{e}-06$ & $7.7 \mathrm{e}-04$ & $-2.4 \mathrm{e}-04$ & $8.1 \mathrm{e}-04$ & 22 & 1163 & 161 & 0.069 & 0.097 \\
5 & $4.4 \mathrm{e}-06$ & $1.2 \mathrm{e}-03$ & $-4.0 \mathrm{e}-05$ & $1.2 \mathrm{e}-03$ & 21 & 979 & 191 & 0.081 & 0.093 \\
6 & $2.3 \mathrm{e}-06$ & $4.6 \mathrm{e}-04$ & $1.9 \mathrm{e}-04$ & $5.0 \mathrm{e}-04$ & 20 & 939 & 165 & 0.103 & 0.092 \\
7 & $9.8 \mathrm{e}-07$ & $3.9 \mathrm{e}-04$ & $3.9 \mathrm{e}-05$ & $3.9 \mathrm{e}-04$ & 22 & 959 & 167 & 0.115 & 0.097 \\
\hline \hline
\end{tabular}

Tabela 4.10: Malha 1: MGC com MRAO de ordem 4 usando diferenças finitas

Prosseguindo, as tabelas 4.11 e 4.12 apresentam, respectivamente, os resultados obtidos, usando recontrução de ordem 4 na malha 2, calculando os gradientes via método adjunto e via diferenças finitas, sendo que a figura 4.11 apresenta a evolução dos parâmetros. O refinamento elevou o custo computacional, porém 


\begin{tabular}{cccccccccc}
\hline \hline it & $\mathcal{F}$ & $\delta_{a} \mathcal{F}$ & $\delta_{b} \mathcal{F}$ & $\|\delta \mathcal{F}\|$ & CP & t(seg) & TCG & a & b \\
\hline \hline 0 & $1.3 \mathrm{e}-03$ & $1.3 \mathrm{e}-02$ & $1.5 \mathrm{e}-02$ & $2.0 \mathrm{e}-02$ & 1 & 343 & 583 & 0.000 & 0.000 \\
1 & $6.4 \mathrm{e}-07$ & $4.7 \mathrm{e}-05$ & $-1.6 \mathrm{e}-04$ & $1.7 \mathrm{e}-04$ & 16 & 1826 & 271 & 0.089 & 0.102 \\
\hline \hline
\end{tabular}

Tabela 4.11: Malha 2: MGC com MRAO de ordem 4 usando método adjunto

\begin{tabular}{cccccccccc}
\hline \hline it & $\mathcal{F}$ & $\delta_{a} \mathcal{F}$ & $\delta_{b} \mathcal{F}$ & $\|\delta \mathcal{F}\|$ & CP & t(seg) & TCG & a & b \\
\hline \hline 0 & $1.3 \mathrm{e}-03$ & $1.6 \mathrm{e}-02$ & $2.7 \mathrm{e}-02$ & $3.2 \mathrm{e}-02$ & 1 & 426 & 1106 & 0.000 & 0.000 \\
1 & $7.1 \mathrm{e}-06$ & $3.0 \mathrm{e}-04$ & $-6.4 \mathrm{e}-04$ & $7.1 \mathrm{e}-04$ & 15 & 2534 & 392 & 0.062 & 0.107 \\
2 & $5.9 \mathrm{e}-06$ & $3.9 \mathrm{e}-04$ & $-4.9 \mathrm{e}-04$ & $6.3 \mathrm{e}-04$ & 21 & 2613 & 413 & 0.064 & 0.102 \\
3 & $5.7 \mathrm{e}-06$ & $5.3 \mathrm{e}-04$ & $-4.2 \mathrm{e}-04$ & $6.8 \mathrm{e}-04$ & 22 & 2599 & 570 & 0.067 & 0.099 \\
4 & $5.6 \mathrm{e}-06$ & $7.2 \mathrm{e}-04$ & $-2.8 \mathrm{e}-04$ & $7.7 \mathrm{e}-04$ & 21 & 2443 & 407 & 0.069 & 0.097 \\
5 & $4.8 \mathrm{e}-06$ & $1.3 \mathrm{e}-03$ & $-1.2 \mathrm{e}-04$ & $1.3 \mathrm{e}-03$ & 21 & 2056 & 589 & 0.080 & 0.093 \\
6 & $3.1 \mathrm{e}-06$ & $9.7 \mathrm{e}-04$ & $2.2 \mathrm{e}-04$ & $9.9 \mathrm{e}-04$ & 20 & 1822 & 621 & 0.101 & 0.091 \\
7 & $1.8 \mathrm{e}-06$ & $3.6 \mathrm{e}-05$ & $8.1 \mathrm{e}-05$ & $8.9 \mathrm{e}-05$ & 20 & 1834 & 497 & 0.114 & 0.093 \\
8 & $9.0 \mathrm{e}-07$ & $7.9 \mathrm{e}-05$ & $2.3 \mathrm{e}-04$ & $2.4 \mathrm{e}-04$ & 25 & 2555 & 531 & 0.116 & 0.098 \\
\hline \hline
\end{tabular}

Tabela 4.12: Malha 2: MGC com MRAO de ordem 4 usando diferenças finitas

ocorreu o mesmo comportamento observado com a malha 1. Mesmo com a difusão numérica agindo, ambos os métodos atingiram a precisão especificada sendo que o método adjunto gerou gradientes mais precisos.

As tabelas 4.13 e 4.14 apresentam, respectivamente, os resultados obtidos, usando recontrução de ordem 4 na malha 3, calculando os gradientes via método adjunto e via diferenças finitas, sendo que o gráfico 4.13 apresenta a evolução dos parâmetros. Nesse caso o processo utilizando o método adjunto exibiu o mesmo comportamento observado para o método de diferenças finitas nas malhas anteriores, requerendo inclusive o mesmo tempo computacional. Acredita-se que isso se deve ao fato dos níveis de tolerância para o primal e adjunto estarem inadequados para esse nível de malha, exigindo portanto uma precisão mais elevada para as soluções do problema primal e adjunto. Essa hipótese será investigada nos estudos de sensibilidade do próximo capítulo.

Note que em todos os casos, o TCG (tempo de cálculo do gradiente) em cada iteração, de um modo geral, é menor utilizando métodos adjuntos do que diferenças finitas. Para dois parâmetros, a diferença entre os TCGs calculados via adjunto e via diferenças finitas não é tão grande, mas a medida que aumenta a quantidade de parâmetros, o TCG entre adjunto e diferença finita aumenta bastante. Os testes feitos com três e quatro parâmetros, apresentados nas próximas seções, mostrarão isso. 


\begin{tabular}{cccccccccc}
\hline \hline it & $\mathcal{F}$ & $\delta_{a} \mathcal{F}$ & $\delta_{b} \mathcal{F}$ & $\|\delta \mathcal{F}\|$ & CP & t(seg) & TCG & a & b \\
\hline \hline 0 & $1.3 \mathrm{e}-03$ & $1.4 \mathrm{e}-02$ & $1.9 \mathrm{e}-02$ & $2.4 \mathrm{e}-02$ & 1 & 927 & 1584 & 0.000 & 0.000 \\
1 & $3.3 \mathrm{e}-06$ & $1.6 \mathrm{e}-04$ & $-3.5 \mathrm{e}-04$ & $3.8 \mathrm{e}-04$ & 15 & 4250 & 417 & 0.078 & 0.106 \\
2 & $1.9 \mathrm{e}-06$ & $2.3 \mathrm{e}-04$ & $-3.7 \mathrm{e}-04$ & $4.4 \mathrm{e}-04$ & 23 & 3965 & 210 & 0.080 & 0.102 \\
3 & $1.7 \mathrm{e}-06$ & $2.7 \mathrm{e}-04$ & $-2.8 \mathrm{e}-04$ & $3.9 \mathrm{e}-04$ & 22 & 3795 & 195 & 0.081 & 0.100 \\
4 & $1.7 \mathrm{e}-06$ & $3.1 \mathrm{e}-04$ & $-2.0 \mathrm{e}-04$ & $3.7 \mathrm{e}-04$ & 22 & 3731 & 178 & 0.083 & 0.098 \\
5 & $1.6 \mathrm{e}-06$ & $4.2 \mathrm{e}-04$ & $-1.4 \mathrm{e}-04$ & $4.4 \mathrm{e}-04$ & 23 & 3538 & 199 & 0.085 & 0.097 \\
6 & $1.4 \mathrm{e}-06$ & $8.1 \mathrm{e}-04$ & $-7.7 \mathrm{e}-05$ & $8.1 \mathrm{e}-04$ & 22 & 3127 & 234 & 0.091 & 0.095 \\
7 & $1.1 \mathrm{e}-06$ & $1.1 \mathrm{e}-03$ & $1.1 \mathrm{e}-04$ & $1.1 \mathrm{e}-03$ & 21 & 2550 & 200 & 0.101 & 0.094 \\
8 & $8.5 \mathrm{e}-07$ & $9.5 \mathrm{e}-04$ & $2.9 \mathrm{e}-04$ & $9.9 \mathrm{e}-04$ & 20 & 2334 & 324 & 0.106 & 0.095 \\
\hline \hline
\end{tabular}

Tabela 4.13: Malha 3: MGC com MRAO de ordem 4 usando método adjunto

\begin{tabular}{cccccccccc}
\hline \hline it & $\mathcal{F}$ & $\delta_{a} \mathcal{F}$ & $\delta_{b} \mathcal{F}$ & $\|\delta \mathcal{F}\|$ & CP & t(seg) & TCG & a & b \\
\hline \hline 0 & $1.3 \mathrm{e}-03$ & $1.6 \mathrm{e}-02$ & $2.8 \mathrm{e}-02$ & $3.2 \mathrm{e}-02$ & 1 & 892 & 2171 & 0.000 & 0.000 \\
1 & $7.6 \mathrm{e}-06$ & $3.1 \mathrm{e}-04$ & $-6.2 \mathrm{e}-04$ & $7.0 \mathrm{e}-04$ & 15 & 4812 & 761 & 0.062 & 0.107 \\
2 & $6.1 \mathrm{e}-06$ & $4.1 \mathrm{e}-04$ & $-4.8 \mathrm{e}-04$ & $6.3 \mathrm{e}-04$ & 21 & 4051 & 743 & 0.064 & 0.102 \\
3 & $5.8 \mathrm{e}-06$ & $5.4 \mathrm{e}-04$ & $-3.5 \mathrm{e}-04$ & $6.5 \mathrm{e}-04$ & 22 & 4520 & 815 & 0.067 & 0.099 \\
4 & $5.4 \mathrm{e}-06$ & $7.7 \mathrm{e}-04$ & $-2.0 \mathrm{e}-04$ & $8.0 \mathrm{e}-04$ & 22 & 3780 & 718 & 0.071 & 0.096 \\
5 & $3.8 \mathrm{e}-06$ & $1.2 \mathrm{e}-03$ & $6.1 \mathrm{e}-05$ & $1.2 \mathrm{e}-03$ & 21 & 2810 & 698 & 0.090 & 0.091 \\
6 & $2.1 \mathrm{e}-06$ & $3.3 \mathrm{e}-04$ & $2.1 \mathrm{e}-04$ & $3.9 \mathrm{e}-04$ & 20 & 2350 & 662 & 0.103 & 0.092 \\
7 & $5.8 \mathrm{e}-07$ & $1.4 \mathrm{e}-04$ & $1.5 \mathrm{e}-05$ & $1.4 \mathrm{e}-04$ & 22 & 2655 & 527 & 0.110 & 0.096 \\
\hline \hline
\end{tabular}

Tabela 4.14: Malha 3: MGC com MRAO de ordem 4 usando diferenças finitas 


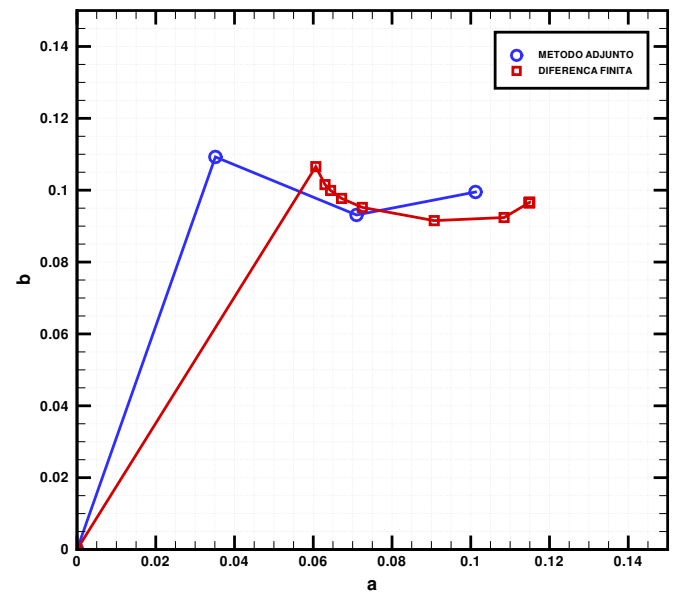

Figura 4.8: Malha 1 - MGC - MRAO - ordem 3

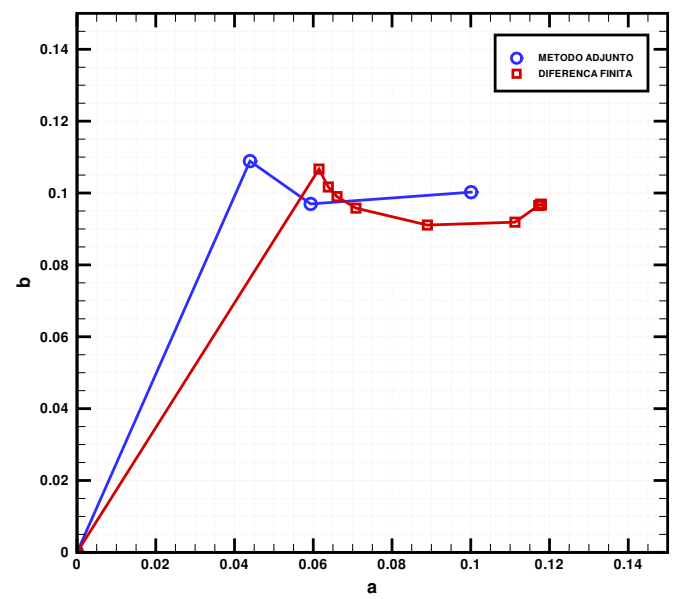

Figura 4.10: Malha 2 - MGC - MRAO - ordem 3

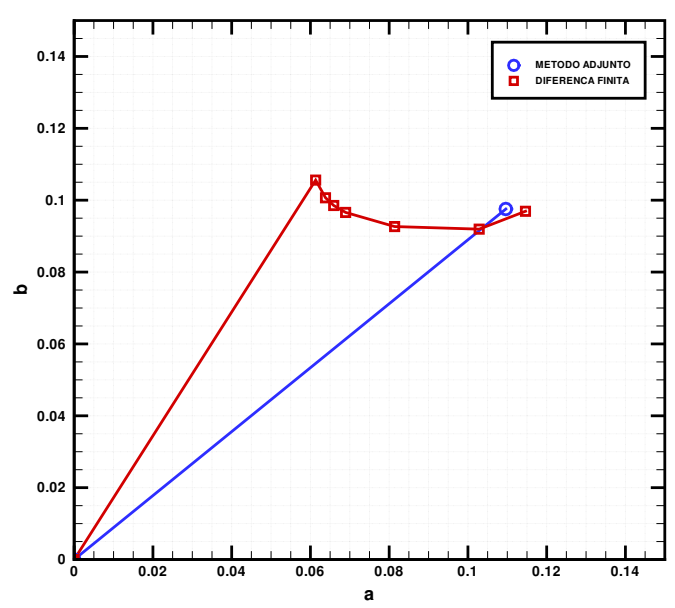

Figura 4.9: Malha 1 - MGC - MRAO - ordem 4

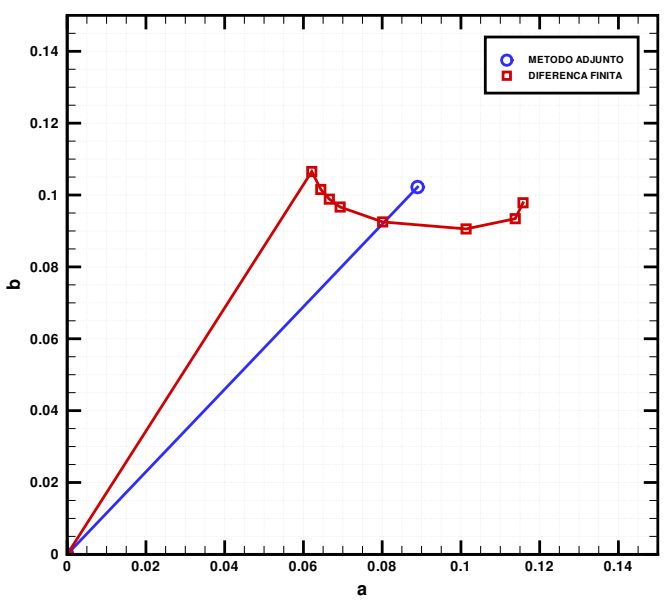

Figura 4.11: Malha 2 - MGC - MRAO - ordem 4 


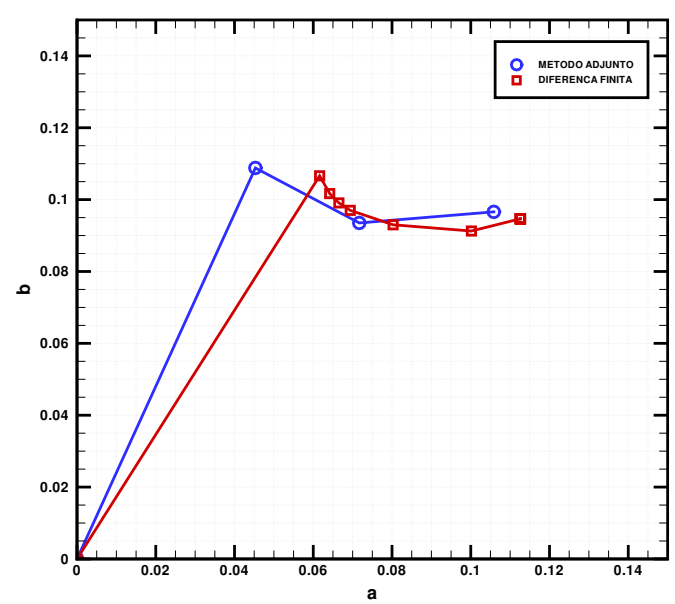

Figura 4.12: Malha 3 - MGC - MRAO - ordem 3

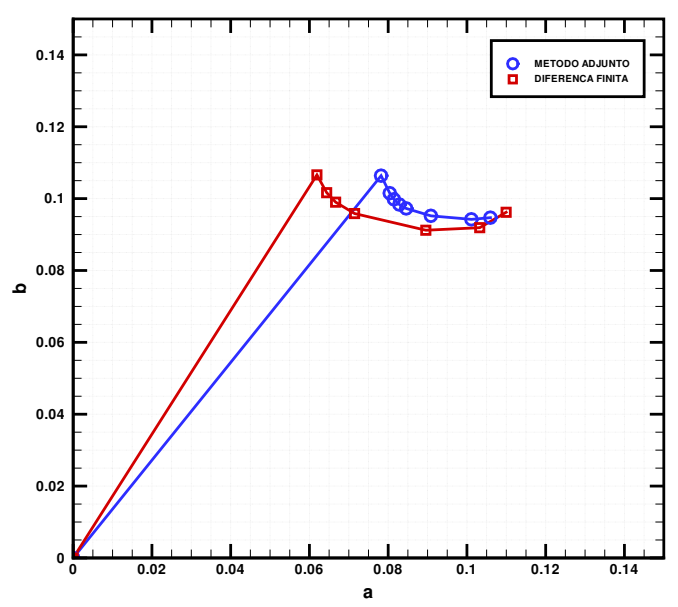

Figura 4.13: Malha 3 - MGC - MRAO - ordem 4

\subsection{Problema teste 2: estimação de três parâmetros - função quadrática}

Este teste consiste em estimar $a, b$ e $c$ da função $k(x, y)=a x^{2}+b x y+c y^{2}+C_{0}$, com $C_{0}=0.05$, que define o coeficiente de difusão na equação de advecção-difusão. Todo o processo é realizado utilizando o mesmo problema de otimização no teste da seção anterior. A $\phi_{\mathrm{R}}$ é obtida utilizando $a_{\mathrm{R}}=b_{\mathrm{R}}=c_{\mathrm{R}}=0.1$. O valor máximo que $k(x, y)$ pode assumir no domínio é 0.35 . Os parâmetros iniciais foram $a=b=c=0$. Logo, ao executar o método, os parâmetros devem convergir para $a_{\mathrm{R}}=b_{\mathrm{R}}=c_{\mathrm{R}}=0.1$. Para calcular os gradientes por diferenças finitas tomou-se $\delta a=\delta b=\delta c=0.01$.

As figuras 4.14, 4.15, 4.16 e 4.17 apresentam, respectivamente, a solução de referência $\phi_{R}$, a função $k(x, y)$ de referência ( onde $a_{\mathrm{R}}=b_{\mathrm{R}}=c_{\mathrm{R}}=0.1$ ), termo fonte $\phi_{\mathrm{R}}-\phi$ do problema adjunto inicial e a solução inicial $\psi$ do problema adjunto. Assim como no teste 1 , note que os valores do termo fonte são pequenos, assim como da solução do problema adjunto inicial. Esses valores tendem a diminuir cada vez mais, pois a medida que os parâmetros se aproximam dos parâmetros ótimos, a solução do problema adjunto tende a zero, uma vez que o termo fonte do problema adjunto é dado por $\phi-\phi_{R}$.

A tabela 4.15 apresenta uma descrição das colunas das tabelas de resultados. Reiterando, nas tabelas de resultados, MGC é abreviação de Método dos Gradientes Conjugados e MRAO de Método de Reconstrução de Alta Ordem.

As tabelas 4.16 e 4.17 apresentam, respectivamente, os resultados obtidos, usando recontrução de ordem 


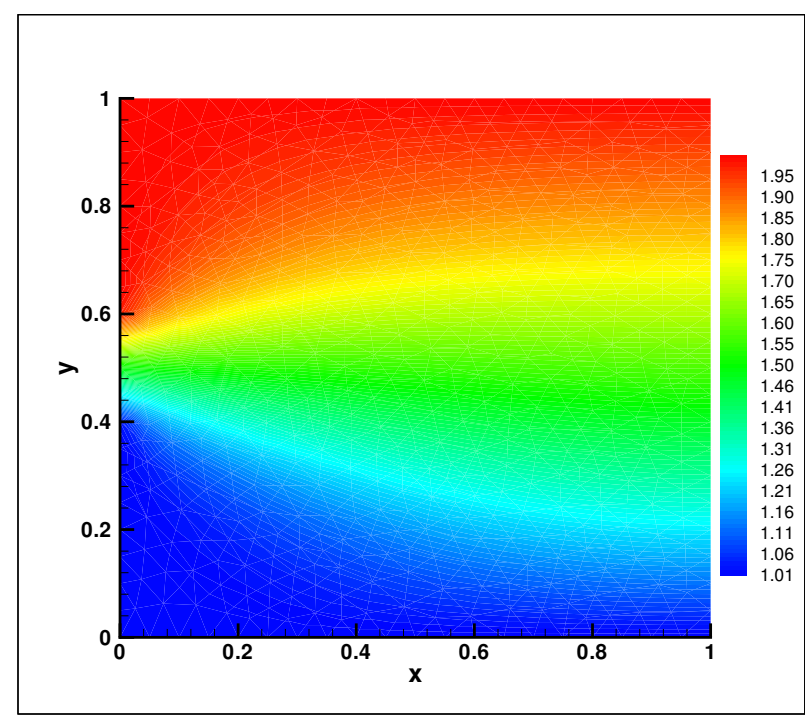

Figura 4.14: Gráfico de $\phi_{\mathrm{R}}$

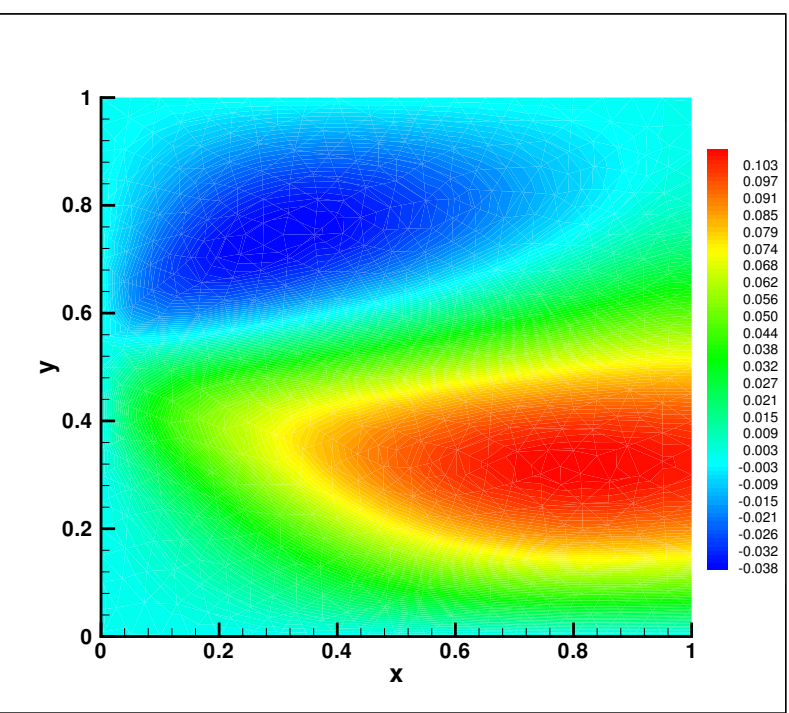

Figura 4.16: Termo fonte do dual inicial $\phi_{R}-\phi$

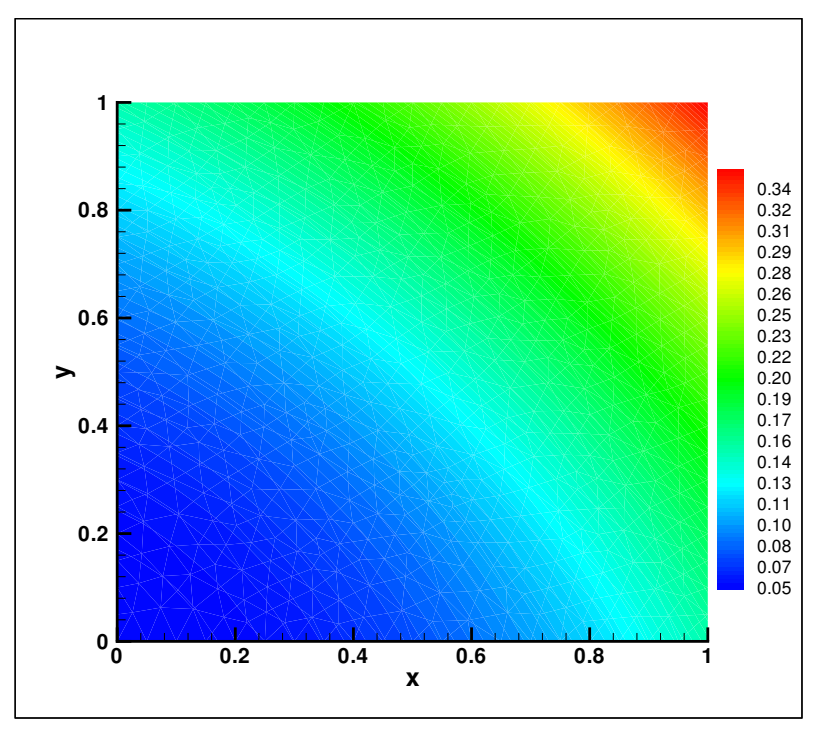

Figura 4.15: Função de referência

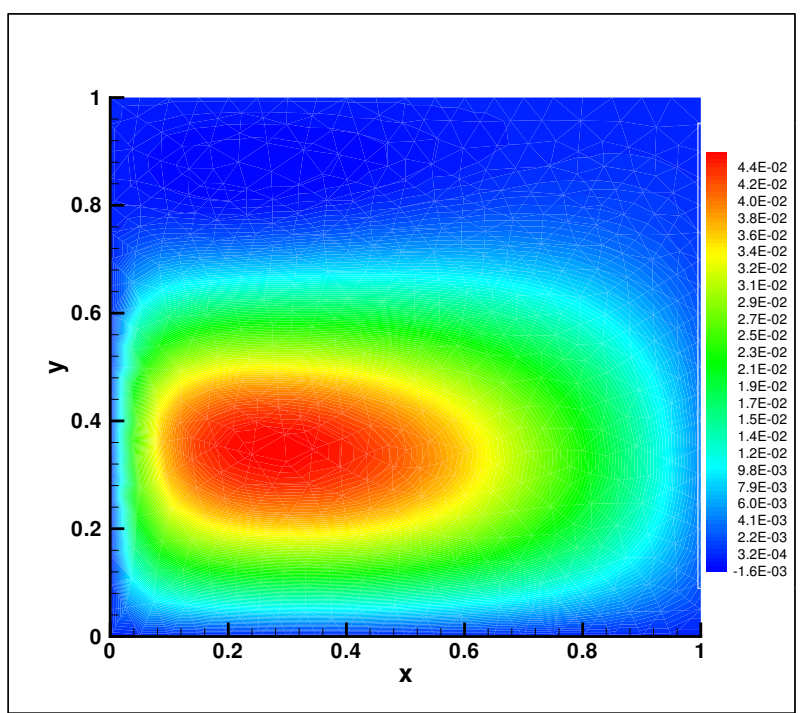

Figura 4.17: Gráfico da solução dual inicial $\psi$ 


\begin{tabular}{|c|c|l|}
\hline $1^{a}$ & it & iterações \\
\hline $2^{a}$ & $\mathcal{F}$ & valor do funcional \\
\hline $3^{a}$ & $\delta_{a} \mathcal{F}$ & gradiente em relação a $a$ \\
\hline $4^{a}$ & $\delta_{b} \mathcal{F}$ & gradiente em relação a $b$ \\
\hline $5^{a}$ & $\delta_{c} \mathcal{F}$ & gradiente em relação a $c$ \\
\hline $6^{a}$ & $\|\delta \mathcal{F}\|$ & norma 2 do gradiente \\
\hline $7^{a}$ & $\mathrm{CP}$ & número de chamadas do primal \\
\hline $8^{a}$ & $\mathrm{t}(\mathrm{seg})$ & tempo gasto na busca unidirecional (em segundos) \\
\hline $9^{a}$ & $\mathrm{TCG}$ & tempo de cálculo do gradiente (em segundos) \\
\hline $10^{a}$ & $\mathrm{a}$ & estimativa do primeiro parâmetro \\
\hline $11^{a}$ & $\mathrm{~b}$ & estimativa do segundo parâmetro \\
\hline $12^{a}$ & $\mathrm{c}$ & estimativa do terceiro parâmetro \\
\hline
\end{tabular}

Tabela 4.15: Teste 2: Descrição das tabelas

3 na malha 1, calculando os gradientes via método adjunto e via diferenças finitas, e as tabelas 4.18 e 4.19 apresentam, respectivamente, os resultados obtidos, usando recontrução de ordem 4 na malha 1, calculando os gradientes via método adjunto e via diferenças finitas. Percebe-se nesse caso que o método adjunto não foi efetivo em comparação com o método de diferenças finitas. Comparando-se as duas tabelas não existe uma diferença significativa de qualidade, sendo inclusive o método adjunto um pouco pior. Aumentando para quarta ordem na mesma malha 1 , como apresentado na tabela 4.18, observa-se um agravamento na capacidade de convergência do método adjunto, sendo esse incapaz de atingir a precisão especificada antes do número máximo de iterações. Já o método de diferenças finitas apresenta um comportamento bem mais adequado.

\begin{tabular}{cccccccccccc}
\hline \hline it & $\mathcal{F}$ & $\delta_{a} \mathcal{F}$ & $\delta_{b} \mathcal{F}$ & $\delta_{c} \mathcal{F}$ & $\|\delta \mathcal{F}\|$ & CP & t(seg) & TCG & a & b & c \\
\hline \hline 0 & $1.2 \mathrm{e}-03$ & $5.7 \mathrm{e}-03$ & $1.6 \mathrm{e}-02$ & $5.7 \mathrm{e}-02$ & $5.9 \mathrm{e}-02$ & 1 & 23 & 40 & 0.000 & 0.000 & 0.000 \\
1 & $4.7 \mathrm{e}-05$ & $8.6 \mathrm{e}-04$ & $1.1 \mathrm{e}-03$ & $-3.3 \mathrm{e}-05$ & $1.4 \mathrm{e}-03$ & 13 & 167 & 30 & 0.014 & 0.038 & 0.137 \\
2 & $3.0 \mathrm{e}-05$ & $3.5 \mathrm{e}-03$ & $3.5 \mathrm{e}-03$ & $-2.5 \mathrm{e}-03$ & $5.5 \mathrm{e}-03$ & 21 & 239 & 23 & 0.040 & 0.070 & 0.136 \\
3 & $3.1 \mathrm{e}-06$ & $6.5 \mathrm{e}-04$ & $2.1 \mathrm{e}-04$ & $-1.3 \mathrm{e}-03$ & $1.5 \mathrm{e}-03$ & 18 & 203 & 29 & 0.099 & 0.129 & 0.094 \\
4 & $1.4 \mathrm{e}-06$ & $5.1 \mathrm{e}-06$ & $-1.4 \mathrm{e}-04$ & $1.2 \mathrm{e}-05$ & $1.4 \mathrm{e}-04$ & 20 & 229 & 20 & 0.104 & 0.130 & 0.086 \\
5 & $1.0 \mathrm{e}-06$ & $1.8 \mathrm{e}-06$ & $-1.4 \mathrm{e}-03$ & $5.8 \mathrm{e}-04$ & $1.5 \mathrm{e}-03$ & 25 & 273 & 18 & 0.104 & 0.122 & 0.086 \\
6 & $5.1 \mathrm{e}-07$ & $-6.1 \mathrm{e}-05$ & $-1.5 \mathrm{e}-03$ & $1.2 \mathrm{e}-03$ & $1.9 \mathrm{e}-03$ & 20 & 208 & 17 & 0.104 & 0.104 & 0.094 \\
\hline \hline
\end{tabular}

Tabela 4.16: Malha 1: MGC com MRAO de ordem 3 usando método adjunto - 3 parâmetros

As tabelas 4.4 e 4.21 apresentam, respectivamente, os resultados obtidos, usando recontrução de ordem 3 na malha 2, calculando os gradientes via método adjunto e via diferenças finitas, e as tabelas 4.22 e 4.23 apresentam, respectivamente, os resultados obtidos, usando recontrução de ordem 4 na malha 2, calculando 


\begin{tabular}{cccccccccccc}
\hline \hline it & $\mathcal{F}$ & $\delta_{a} \mathcal{F}$ & $\delta_{b} \mathcal{F}$ & $\delta_{c} \mathcal{F}$ & $\|\delta \mathcal{F}\|$ & CP & t(seg) & TCG & a & b & c \\
\hline \hline 0 & $1.3 \mathrm{e}-03$ & $6.5 \mathrm{e}-03$ & $1.1 \mathrm{e}-02$ & $1.7 \mathrm{e}-02$ & $2.1 \mathrm{e}-02$ & 1 & 24 & 65 & 0.000 & 0.000 & 0.000 \\
1 & $1.3 \mathrm{e}-05$ & $3.6 \mathrm{e}-04$ & $1.5 \mathrm{e}-05$ & $-5.4 \mathrm{e}-04$ & $6.5 \mathrm{e}-04$ & 16 & 202 & 48 & 0.045 & 0.075 & 0.117 \\
2 & $1.1 \mathrm{e}-05$ & $4.6 \mathrm{e}-04$ & $2.6 \mathrm{e}-04$ & $-2.5 \mathrm{e}-04$ & $5.8 \mathrm{e}-04$ & 22 & 261 & 48 & 0.051 & 0.075 & 0.108 \\
3 & $5.5 \mathrm{e}-06$ & $7.7 \mathrm{e}-04$ & $7.0 \mathrm{e}-04$ & $-1.8 \mathrm{e}-06$ & $1.0 \mathrm{e}-03$ & 22 & 239 & 48 & 0.077 & 0.090 & 0.094 \\
4 & $1.4 \mathrm{e}-07$ & $1.2 \mathrm{e}-04$ & $7.1 \mathrm{e}-05$ & $-2.1 \mathrm{e}-04$ & $2.5 \mathrm{e}-04$ & 21 & 239 & 49 & 0.098 & 0.109 & 0.094 \\
\hline \hline
\end{tabular}

Tabela 4.17: Malha 1: MGC com MRAO de ordem 3 usando diferenças finitas - 3 parâmetros

\begin{tabular}{|c|c|c|c|c|c|c|c|c|c|c|c|}
\hline it & $\overline{\overline{\mathcal{F}}}$ & $\overline{\delta_{a} \mathcal{F}}$ & $\overline{\delta_{b} \mathcal{F}}$ & $\overline{\overline{\delta_{c} \mathcal{F}}}$ & $\overline{\|\| \delta \mathcal{F} \mid}$ & $\overline{C \mathrm{CP}}$ & t(seg) & $\overline{\mathrm{TCG}}$ & a & b & c \\
\hline$\overline{c 0}$ & 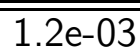 & $\overline{5.1 \mathrm{e}-03}$ & 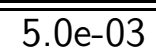 & $=7.4 \mathrm{e}-03$ & 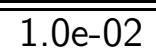 & $\overline{\overline{1}}$ & $\overline{\overline{101}}$ & $\overline{c 178}$ & 0.000 & 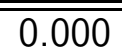 & 0.000 \\
\hline 1 & $3.1 \mathrm{e}-06$ & $2.2 \mathrm{e}-05$ & $-1.5 \mathrm{e}-04$ & $-5.0 e-04$ & $5.2 \mathrm{e}-04$ & 17 & 868 & 110 & 0.079 & 0.076 & 0.114 \\
\hline 2 & $3.0 \mathrm{e}-06$ & $2.1 \mathrm{e}-05$ & $-2.4 \mathrm{e}-04$ & $-8.5 \mathrm{e}-04$ & $8.9 \mathrm{e}-04$ & 22 & 1400 & 148 & 0.079 & 0.075 & 0.113 \\
\hline 3 & $3.0 \mathrm{e}-06$ & $2.4 \mathrm{e}-05$ & $-3.6 \mathrm{e}-04$ & $-1.3 e-03$ & $1.3 \mathrm{e}-03$ & 21 & 1322 & 84 & 0.079 & 0.075 & 0.112 \\
\hline 4 & $3.0 \mathrm{e}-06$ & $3.1 \mathrm{e}-05$ & $-4.6 e-04$ & $-1.7 \mathrm{e}-03$ & $1.7 \mathrm{e}-03$ & 20 & 1020 & 69 & 0.079 & 0.075 & 0.112 \\
\hline 5 & $3.0 \mathrm{e}-06$ & $4.4 \mathrm{e}-05$ & $-5.9 \mathrm{e}-04$ & $-2.1 \mathrm{e}-03$ & 2.2e- 03 & 20 & 1014 & 68 & 0.079 & 0.075 & 0.112 \\
\hline 6 & $3.0 \mathrm{e}-06$ & $4.9 \mathrm{e}-05$ & $-7.0 \mathrm{e}-04$ & $-2.5 e-03$ & 2.6e- -03 & 19 & 963 & 77 & 0.079 & 0.075 & 0.112 \\
\hline 7 & 3.0e-06 & $5.6 \mathrm{e}-05$ & $-8.7 \mathrm{e}-04$ & $-3.1 \mathrm{e}-03$ & $3.3 e-03$ & 19 & 961 & 115 & 0.079 & 0.075 & 0.112 \\
\hline 8 & $3.0 \mathrm{e}-06$ & $5.7 \mathrm{e}-05$ & $-9.3 \mathrm{e}-04$ & $-3.4 \mathrm{e}-03$ & $3.5 \mathrm{e}-03$ & 18 & 947 & 71 & 0.079 & 0.075 & 0.112 \\
\hline 9 & $3.0 \mathrm{e}-06$ & $7.4 \mathrm{e}-05$ & $-1.1 \mathrm{e}-03$ & $-4.0 \mathrm{e}-03$ & $4.1 \mathrm{e}-03$ & 18 & 915 & 69 & 0.079 & 0.075 & 0.112 \\
\hline 10 & $3.0 \mathrm{e}-06$ & $7.9 \mathrm{e}-05$ & $-1.2 \mathrm{e}-03$ & $-4.5 e-03$ & $4.7 \mathrm{e}-03$ & 18 & 915 & 111 & 0.079 & 0.075 & 0.112 \\
\hline 11 & $3.0 \mathrm{e}-06$ & $8.2 \mathrm{e}-05$ & $-1.3 e-03$ & $-4.8 \mathrm{e}-03$ & $5.0 \mathrm{e}-03$ & 18 & 892 & 47 & 0.079 & 0.075 & 0.112 \\
\hline 12 & $3.0 \mathrm{e}-06$ & $8.9 \mathrm{e}-05$ & $-1.5 \mathrm{e}-03$ & $-5.3 e-03$ & $5.5 \mathrm{e}-03$ & 17 & 860 & 47 & 0.079 & 0.075 & 0.112 \\
\hline 13 & $3.0 \mathrm{e}-06$ & $9.7 \mathrm{e}-05$ & $-1.6 \mathrm{e}-03$ & $-5.8 e-03$ & $6.0 \mathrm{e}-03$ & 17 & 847 & 47 & 0.079 & 0.075 & 0.112 \\
\hline 14 & $3.0 \mathrm{e}-06$ & $1.1 \mathrm{e}-04$ & $-1.7 \mathrm{e}-03$ & $-6.3 e-03$ & $6.5 \mathrm{e}-03$ & 17 & 848 & 47 & 0.079 & 0.075 & 0.112 \\
\hline 15 & $3.0 \mathrm{e}-06$ & $1.2 \mathrm{e}-04$ & $-1.8 \mathrm{e}-03$ & $-6.7 e-03$ & 7.0e-03 & 17 & 865 & 47 & 0.079 & 0.075 & 0.112 \\
\hline 16 & $3.0 \mathrm{e}-06$ & $1.2 \mathrm{e}-04$ & $-1.8 \mathrm{e}-03$ & $-6.6 e-03$ & $6.9 \mathrm{e}-03$ & 17 & 885 & 68 & 0.079 & 0.075 & 0.112 \\
\hline 17 & $3.0 \mathrm{e}-06$ & $1.1 \mathrm{e}-04$ & $-1.8 \mathrm{e}-03$ & $-6.6 e-03$ & $6.9 \mathrm{e}-03$ & 17 & 855 & 48 & 0.079 & 0.075 & 0.112 \\
\hline 18 & $3.0 \mathrm{e}-06$ & $1.2 \mathrm{e}-04$ & $-2.0 \mathrm{e}-03$ & $-7.2 \mathrm{e}-03$ & $7.5 \mathrm{e}-03$ & 17 & 842 & 46 & 0.079 & 0.075 & 0.112 \\
\hline 19 & $3.0 \mathrm{e}-06$ & $1.4 \mathrm{e}-04$ & $-2.1 \mathrm{e}-03$ & $-7.8 \mathrm{e}-03$ & $8.1 \mathrm{e}-03$ & 17 & 865 & 46 & 0.079 & 0.075 & 0.112 \\
\hline 20 & $3.0 \mathrm{e}-06$ & $1.5 \mathrm{e}-04$ & $-2.3 e-03$ & $-8.4 \mathrm{e}-03$ & $8.7 \mathrm{e}-03$ & 16 & 803 & 47 & 0.079 & 0.075 & 0.112 \\
\hline
\end{tabular}

Tabela 4.18: Malha 1: MGC com MRAO de ordem 4 usando método adjunto - 3 parâmetros

\begin{tabular}{cccccccccccc}
\hline \hline it & $\mathcal{F}$ & $\delta_{a} \mathcal{F}$ & $\delta_{b} \mathcal{F}$ & $\delta_{c} \mathcal{F}$ & $\|\delta \mathcal{F}\|$ & CP & t(seg) & TCG & a & b & c \\
\hline \hline 0 & $1.2 \mathrm{e}-03$ & $6.3 \mathrm{e}-03$ & $1.1 \mathrm{e}-02$ & $1.6 \mathrm{e}-02$ & $2.0 \mathrm{e}-02$ & 1 & 91 & 243 & 0.000 & 0.000 & 0.000 \\
1 & $1.3 \mathrm{e}-05$ & $3.6 \mathrm{e}-04$ & $2.3 \mathrm{e}-05$ & $-5.2 \mathrm{e}-04$ & $6.3 \mathrm{e}-04$ & 16 & 812 & 192 & 0.044 & 0.075 & 0.116 \\
2 & $1.1 \mathrm{e}-05$ & $4.5 \mathrm{e}-04$ & $2.6 \mathrm{e}-04$ & $-2.5 \mathrm{e}-04$ & $5.8 \mathrm{e}-04$ & 22 & 1245 & 346 & 0.050 & 0.075 & 0.108 \\
3 & $5.8 \mathrm{e}-06$ & $8.6 \mathrm{e}-04$ & $7.7 \mathrm{e}-04$ & $-4.1 \mathrm{e}-05$ & $1.2 \mathrm{e}-03$ & 22 & 1270 & 241 & 0.076 & 0.090 & 0.093 \\
4 & $3.1 \mathrm{e}-07$ & $5.2 \mathrm{e}-05$ & $3.1 \mathrm{e}-05$ & $-1.6 \mathrm{e}-04$ & $1.7 \mathrm{e}-04$ & 21 & 961 & 191 & 0.100 & 0.111 & 0.092 \\
\hline \hline
\end{tabular}

Tabela 4.19: Malha 1: MGC com MRAO de ordem 4 usando diferenças finitas - 3 parâmetros 
os gradientes via método adjunto e via diferenças finitas. Aumentando o refinamento da malha passando para malha 2. O método adjunto recupera o comportamento observado anteriormente, atingindo a convergência com cerca de metade do custo computacional de diferenças finitas. Em ordem 4, observa-se o mesmo comportamento de ordem 3 com o método adjunto consumindo menos da metade do tempo requerido por diferenças finitas para atingir a convergência. Com esses resultados, ao que parece, existe uma relação entre a forma função funcional de $\kappa(x, y)$ e do grau de refinamento da malha para que o método adjunto funcione bem. O refinamento ajudou o método adjunto a se comportar melhor e a atingir a convergência.

\begin{tabular}{cccccccccccc}
\hline \hline it & $\mathcal{F}$ & $\delta_{a} \mathcal{F}$ & $\delta_{b} \mathcal{F}$ & $\delta_{c} \mathcal{F}$ & $\|\delta \mathcal{F}\|$ & CP & t(seg) & TCG & a & b & c \\
\hline \hline 0 & $1.2 \mathrm{e}-03$ & $6.4 \mathrm{e}-03$ & $1.4 \mathrm{e}-02$ & $4.1 \mathrm{e}-02$ & $4.4 \mathrm{e}-02$ & 1 & 98 & 162 & 0.000 & 0.000 & 0.000 \\
1 & $3.8 \mathrm{e}-05$ & $6.9 \mathrm{e}-04$ & $5.8 \mathrm{e}-04$ & $-5.1 \mathrm{e}-04$ & $1.0 \mathrm{e}-03$ & 14 & 471 & 79 & 0.021 & 0.045 & 0.135 \\
2 & $4.9 \mathrm{e}-06$ & $8.9 \mathrm{e}-04$ & $1.1 \mathrm{e}-03$ & $4.4 \mathrm{e}-04$ & $1.5 \mathrm{e}-03$ & 22 & 482 & 47 & 0.077 & 0.091 & 0.094 \\
3 & $3.9 \mathrm{e}-07$ & $9.0 \mathrm{e}-05$ & $5.9 \mathrm{e}-05$ & $8.5 \mathrm{e}-05$ & $1.4 \mathrm{e}-04$ & 20 & 460 & 48 & 0.086 & 0.103 & 0.099 \\
\hline \hline
\end{tabular}

Tabela 4.20: Malha 2: MGC com MRAO de ordem 3 usando método adjunto - 3 parâmetros

\begin{tabular}{cccccccccccc}
\hline \hline it & $\mathcal{F}$ & $\delta_{a} \mathcal{F}$ & $\delta_{b} \mathcal{F}$ & $\delta_{c} \mathcal{F}$ & $\|\delta \mathcal{F}\|$ & CP & t(seg) & TCG & a & b & c \\
\hline \hline 0 & $1.3 \mathrm{e}-03$ & $6.6 \mathrm{e}-03$ & $1.1 \mathrm{e}-02$ & $1.7 \mathrm{e}-02$ & $2.1 \mathrm{e}-02$ & 1 & 117 & 432 & 0.000 & 0.000 & 0.000 \\
1 & $1.2 \mathrm{e}-05$ & $3.5 \mathrm{e}-04$ & $-7.7 \mathrm{e}-06$ & $-5.7 \mathrm{e}-04$ & $6.7 \mathrm{e}-04$ & 16 & 726 & 227 & 0.046 & 0.076 & 0.117 \\
2 & $1.0 \mathrm{e}-05$ & $4.3 \mathrm{e}-04$ & $2.3 \mathrm{e}-04$ & $-2.6 \mathrm{e}-04$ & $5.5 \mathrm{e}-04$ & 22 & 816 & 231 & 0.051 & 0.076 & 0.108 \\
3 & $6.0 \mathrm{e}-06$ & $8.7 \mathrm{e}-04$ & $7.5 \mathrm{e}-04$ & $-7.8 \mathrm{e}-05$ & $1.2 \mathrm{e}-03$ & 23 & 542 & 169 & 0.075 & 0.088 & 0.095 \\
4 & $3.4 \mathrm{e}-07$ & $2.0 \mathrm{e}-05$ & $1.8 \mathrm{e}-05$ & $-1.3 \mathrm{e}-04$ & $1.3 \mathrm{e}-04$ & 21 & 466 & 169 & 0.100 & 0.110 & 0.092 \\
\hline \hline
\end{tabular}

Tabela 4.21: Malha 2: MGC com MRAO de ordem 3 usando diferenças finitas - 3 parâmetros

\begin{tabular}{cccccccccccc}
\hline \hline it & $\mathcal{F}$ & $\delta_{a} \mathcal{F}$ & $\delta_{b} \mathcal{F}$ & $\delta_{c} \mathcal{F}$ & $\|\delta \mathcal{F}\|$ & CP & t $($ seg $)$ & TCG & a & b & c \\
\hline \hline 0 & $1.2 \mathrm{e}-03$ & $5.8 \mathrm{e}-03$ & $6.7 \mathrm{e}-03$ & $7.4 \mathrm{e}-03$ & $1.2 \mathrm{e}-02$ & 1 & 425 & 737 & 0.000 & 0.000 & 0.000 \\
1 & $1.1 \mathrm{e}-06$ & $8.2 \mathrm{e}-05$ & $-4.6 \mathrm{e}-05$ & $-2.0 \mathrm{e}-04$ & $2.2 \mathrm{e}-04$ & 17 & 2366 & 191 & 0.082 & 0.094 & 0.104 \\
2 & $9.3 \mathrm{e}-07$ & $7.1 \mathrm{e}-05$ & $-4.8 \mathrm{e}-07$ & $-1.0 \mathrm{e}-04$ & $1.2 \mathrm{e}-04$ & 24 & 2342 & 118 & 0.083 & 0.094 & 0.102 \\
\hline \hline
\end{tabular}

Tabela 4.22: Malha 2: MGC com MRAO de ordem 4 usando método adjunto - 3 parâmetros

As tabelas 4.24 e 4.25 apresentam, respectivamente, os resultados obtidos, usando recontrução de ordem 3 na malha 3, calculando os gradientes via método adjunto e via diferenças finitas, e as tabelas 4.26 e 4.27 apresentam, respectivamente, os resultados obtidos, usando recontrução de ordem 4 na malha 3, calculando os gradientes via método adjunto e via diferenças finitas. No refinamento da malha observa-se em ordem 3 a mesma eficiência computacional esperada do método adjunto. Entretanto, na malha 3, em ordem 4 tem-se 


\begin{tabular}{cccccccccccc}
\hline \hline it & $\mathcal{F}$ & $\delta_{a} \mathcal{F}$ & $\delta_{b} \mathcal{F}$ & $\delta_{c} \mathcal{F}$ & $\|\delta \mathcal{F}\|$ & CP & t(seg) & TCG & a & b & c \\
\hline \hline 0 & $1.2 \mathrm{e}-03$ & $6.5 \mathrm{e}-03$ & $1.1 \mathrm{e}-02$ & $1.6 \mathrm{e}-02$ & $2.1 \mathrm{e}-02$ & 1 & 342 & 802 & 0.000 & 0.000 & 0.000 \\
1 & $1.2 \mathrm{e}-05$ & $3.5 \mathrm{e}-04$ & $-1.0 \mathrm{e}-05$ & $-6.3 \mathrm{e}-04$ & $7.2 \mathrm{e}-04$ & 16 & 2091 & 617 & 0.046 & 0.076 & 0.117 \\
2 & $1.0 \mathrm{e}-05$ & $4.2 \mathrm{e}-04$ & $1.9 \mathrm{e}-04$ & $-3.4 \mathrm{e}-04$ & $5.7 \mathrm{e}-04$ & 22 & 2313 & 784 & 0.050 & 0.075 & 0.109 \\
3 & $8.4 \mathrm{e}-06$ & $8.7 \mathrm{e}-04$ & $6.5 \mathrm{e}-04$ & $-2.7 \mathrm{e}-04$ & $1.1 \mathrm{e}-03$ & 22 & 2155 & 777 & 0.061 & 0.080 & 0.100 \\
4 & $1.6 \mathrm{e}-06$ & $3.7 \mathrm{e}-05$ & $1.6 \mathrm{e}-04$ & $8.6 \mathrm{e}-05$ & $1.8 \mathrm{e}-04$ & 21 & 1715 & 753 & 0.101 & 0.110 & 0.088 \\
5 & $4.3 \mathrm{e}-07$ & $2.5 \mathrm{e}-05$ & $2.3 \mathrm{e}-04$ & $-2.2 \mathrm{e}-05$ & $2.4 \mathrm{e}-04$ & 24 & 2187 & 747 & 0.102 & 0.115 & 0.091 \\
\hline \hline
\end{tabular}

Tabela 4.23: Malha 2: MGC com MRAO de ordem 4 usando diferenças finitas - 3 parâmetros

o mesmo efeito observado para o caso da difusão linear com dois parâmetros. Método adjunto e o método de diferenças finitas se equivalem. Além disso, esses resultados parecem indicar que a forma funcional de $\kappa(x, y)$ e o grau de refinamento da malha estão relacionadas. No caso do teste 1 , onde a função $k(x, y)$ é uma função linear, não houve problemas de comportamento do adjunto nas três malhas. Para o corrente caso, $k(x, y)$ é uma função quadrática e o refinamento mostrou-se necessário para o método adjunto.

\begin{tabular}{cccccccccccc}
\hline \hline it & $\mathcal{F}$ & $\delta_{a} \mathcal{F}$ & $\delta_{b} \mathcal{F}$ & $\delta_{c} \mathcal{F}$ & $\|\delta \mathcal{F}\|$ & CP & t(seg) & TCG & a & b & c \\
\hline \hline 0 & $1.2 \mathrm{e}-03$ & $6.4 \mathrm{e}-03$ & $1.3 \mathrm{e}-02$ & $3.4 \mathrm{e}-02$ & $3.7 \mathrm{e}-02$ & 1 & 194 & 330 & 0.000 & 0.000 & 0.000 \\
1 & $3.4 \mathrm{e}-05$ & $6.6 \mathrm{e}-04$ & $4.9 \mathrm{e}-04$ & $-2.4 \mathrm{e}-04$ & $8.6 \mathrm{e}-04$ & 14 & 943 & 86 & 0.025 & 0.048 & 0.131 \\
2 & $1.3 \mathrm{e}-06$ & $2.0 \mathrm{e}-04$ & $-4.5 \mathrm{e}-05$ & $-4.6 \mathrm{e}-04$ & $5.0 \mathrm{e}-04$ & 22 & 656 & 66 & 0.104 & 0.107 & 0.103 \\
3 & $1.7 \mathrm{e}-07$ & $1.1 \mathrm{e}-05$ & $-1.7 \mathrm{e}-05$ & $-8.4 \mathrm{e}-06$ & $2.2 \mathrm{e}-05$ & 22 & 866 & 29 & 0.107 & 0.107 & 0.096 \\
\hline \hline
\end{tabular}

Tabela 4.24: Malha 3: MGC com MRAO de ordem 3 usando método adjunto - 3 parâmetros

\begin{tabular}{cccccccccccc}
\hline \hline it & $\mathcal{F}$ & $\delta_{a} \mathcal{F}$ & $\delta_{b} \mathcal{F}$ & $\delta_{c} \mathcal{F}$ & $\|\delta \mathcal{F}\|$ & CP & t(seg) & TCG & a & b & c \\
\hline \hline 0 & $1.3 \mathrm{e}-03$ & $6.7 \mathrm{e}-03$ & $1.1 \mathrm{e}-02$ & $1.7 \mathrm{e}-02$ & $2.1 \mathrm{e}-02$ & 1 & 226 & 506 & 0.000 & 0.000 & 0.000 \\
1 & $1.3 \mathrm{e}-05$ & $3.6 \mathrm{e}-04$ & $2.0 \mathrm{e}-05$ & $-5.3 \mathrm{e}-04$ & $6.4 \mathrm{e}-04$ & 16 & 1100 & 393 & 0.047 & 0.076 & 0.117 \\
2 & $9.9 \mathrm{e}-06$ & $4.4 \mathrm{e}-04$ & $2.8 \mathrm{e}-04$ & $-1.9 \mathrm{e}-04$ & $5.5 \mathrm{e}-04$ & 22 & 1177 & 243 & 0.053 & 0.076 & 0.107 \\
3 & $2.6 \mathrm{e}-06$ & $2.5 \mathrm{e}-04$ & $3.5 \mathrm{e}-04$ & $1.4 \mathrm{e}-04$ & $4.5 \mathrm{e}-04$ & 23 & 795 & 229 & 0.095 & 0.103 & 0.089 \\
4 & $2.5 \mathrm{e}-07$ & $9.8 \mathrm{e}-05$ & $1.1 \mathrm{e}-04$ & $-1.4 \mathrm{e}-04$ & $2.0 \mathrm{e}-04$ & 22 & 1029 & 380 & 0.102 & 0.113 & 0.093 \\
\hline \hline
\end{tabular}

Tabela 4.25: Malha 3: MGC com MRAO de ordem 3 usando diferenças finitas - 3 parâmetros

Note que em todos os casos, o custo computacional para calcular os gradientes, em cada iteração, foi menor nos métodos adjuntos do que com diferenças finitas. Um outro detalhe é que quando os parâmetros $a$, $b$ e $c$ se aproximam de $a_{\mathrm{R}}=b_{\mathrm{R}}=c_{\mathrm{R}}=0.1$, o termo fonte do adjunto tende a se anular. Devido a isso e mais as três condições de fronteira serem nulas $(\psi=0)$, o método adjunto tende a ter um tempo de execução menor. Perceba que quando o problema adjunto tem termo fonte totalmente nulo, a solução no domínio é nula, isto é $\psi=0$. 


\begin{tabular}{cccccccccccc}
\hline \hline it & $\mathcal{F}$ & $\delta_{a} \mathcal{F}$ & $\delta_{b} \mathcal{F}$ & $\delta_{c} \mathcal{F}$ & $\|\delta \mathcal{F}\|$ & CP & t(seg) & TCG & a & b & c \\
\hline \hline 0 & $1.2 \mathrm{e}-03$ & $6.0 \mathrm{e}-03$ & $7.9 \mathrm{e}-03$ & $8.9 \mathrm{e}-03$ & $1.3 \mathrm{e}-02$ & 1 & 784 & 1334 & 0.000 & 0.000 & 0.000 \\
1 & $2.7 \mathrm{e}-06$ & $1.3 \mathrm{e}-04$ & $-4.1 \mathrm{e}-05$ & $-1.8 \mathrm{e}-04$ & $2.3 \mathrm{e}-04$ & 17 & 4041 & 251 & 0.072 & 0.094 & 0.106 \\
2 & $2.0 \mathrm{e}-06$ & $1.4 \mathrm{e}-04$ & $1.7 \mathrm{e}-05$ & $-1.0 \mathrm{e}-04$ & $1.8 \mathrm{e}-04$ & 24 & 3858 & 203 & 0.075 & 0.093 & 0.103 \\
3 & $1.7 \mathrm{e}-06$ & $2.3 \mathrm{e}-04$ & $6.7 \mathrm{e}-05$ & $-1.2 \mathrm{e}-04$ & $2.6 \mathrm{e}-04$ & 25 & 3715 & 148 & 0.079 & 0.094 & 0.100 \\
4 & $1.5 \mathrm{e}-06$ & $4.5 \mathrm{e}-04$ & $2.1 \mathrm{e}-04$ & $-1.2 \mathrm{e}-04$ & $5.1 \mathrm{e}-04$ & 24 & 3302 & 206 & 0.083 & 0.095 & 0.097 \\
5 & $9.1 \mathrm{e}-07$ & $8.6 \mathrm{e}-04$ & $5.2 \mathrm{e}-04$ & $-6.1 \mathrm{e}-05$ & $1.0 \mathrm{e}-03$ & 22 & 2555 & 212 & 0.102 & 0.104 & 0.092 \\
\hline \hline
\end{tabular}

Tabela 4.26: Malha 3: MGC com MRAO de ordem 4 usando método adjunto - 3 parâmetros

\begin{tabular}{cccccccccccc}
\hline \hline it & $\mathcal{F}$ & $\delta_{a} \mathcal{F}$ & $\delta_{b} \mathcal{F}$ & $\delta_{c} \mathcal{F}$ & $\|\delta \mathcal{F}\|$ & CP & t(seg) & TCG & a & b & c \\
\hline \hline 0 & $1.2 \mathrm{e}-03$ & $6.6 \mathrm{e}-03$ & $1.1 \mathrm{e}-02$ & $1.7 \mathrm{e}-02$ & $2.2 \mathrm{e}-02$ & 1 & 792 & 2169 & 0.000 & 0.000 & 0.000 \\
1 & $1.3 \mathrm{e}-05$ & $3.7 \mathrm{e}-04$ & $1.9 \mathrm{e}-05$ & $-5.4 \mathrm{e}-04$ & $6.6 \mathrm{e}-04$ & 16 & 4318 & 873 & 0.045 & 0.076 & 0.117 \\
2 & $1.0 \mathrm{e}-05$ & $4.5 \mathrm{e}-04$ & $2.8 \mathrm{e}-04$ & $-2.0 \mathrm{e}-04$ & $5.6 \mathrm{e}-04$ & 22 & 4323 & 891 & 0.051 & 0.077 & 0.107 \\
3 & $3.0 \mathrm{e}-06$ & $3.3 \mathrm{e}-04$ & $4.1 \mathrm{e}-04$ & $1.4 \mathrm{e}-04$ & $5.5 \mathrm{e}-04$ & 23 & 3206 & 1059 & 0.093 & 0.103 & 0.088 \\
4 & $3.3 \mathrm{e}-07$ & $4.8 \mathrm{e}-05$ & $4.7 \mathrm{e}-05$ & $-1.3 \mathrm{e}-04$ & $1.5 \mathrm{e}-04$ & 22 & 3716 & 807 & 0.101 & 0.113 & 0.091 \\
\hline \hline
\end{tabular}

Tabela 4.27: Malha 3: MGC com MRAO de ordem 4 usando diferenças finitas - 3 parâmetros

\subsection{Problema teste 3: estimação de quatro parâmetros - função quadrática}

Este teste consiste em estimar $a, b, c$ e $d$ na função $k(x, y)=a x+b y+c x(1-x)+d y(1-y)+C_{0}$, com $C_{0}=0.05$, que define o coeficiente de difusão na equação de advecção-difusão. $\mathrm{A} \phi_{\mathrm{R}}$ foi obtida utilizando $a_{\mathrm{R}}=b_{\mathrm{R}}=c_{\mathrm{R}}=d_{\mathrm{R}}=0.1$. O valor máximo que $k(x, y)$ pode assumir então é 0.25 . Os parâmetros iniciais foram $a=b=c=d=0$. Logo, ao executar o método, os parâmetros devem convergir para $a_{\mathrm{R}}=b_{\mathrm{R}}=$ $c_{\mathrm{R}}=d_{\mathrm{R}}=0.1$. Para calcular os gradientes por diferenças finitas tomou-se $\delta a=\delta b=\delta c=\delta d=0.01$.

Os gráficos 4.18, 4.19, 4.20 e 4.21 apresentam, respectivamente, a solução de referência $\phi_{R}$, a função $k(x, y)$ de referência ( onde $a_{\mathrm{R}}=b_{\mathrm{R}}=c_{\mathrm{R}}=c_{\mathrm{R}}=0.1$ ), termo fonte $\phi_{\mathrm{R}}-\phi$ do problema adjunto inicial e a solução inicial $\psi$ do problema adjunto. Assim como nos teste 1 e 2 , note que os valores do termo fonte são pequenos, assim como da solução do problema adjunto inicial. Esses valores tendem a diminuir cada vez mais, pois a medida que os parâmetros se aproximam dos parâmetros ótimos, a solução do problema adjunto tende a zero, uma vez que o termo fonte do problema adjunto é dado por $\phi-\phi_{R}$.

A tabela 4.28 apresenta uma descrição das colunas das tabelas de resultados. Reiterando, nas tabelas de resultados, MGC é abreviação de Método dos Gradientes Conjugados e MRAO de Método de Reconstrução de Alta Ordem.

Uma observação a ser feita aqui é que os parâmetros $a$ e $c$ e os parâmetros $b$ e $d$ estão correlacionados, 


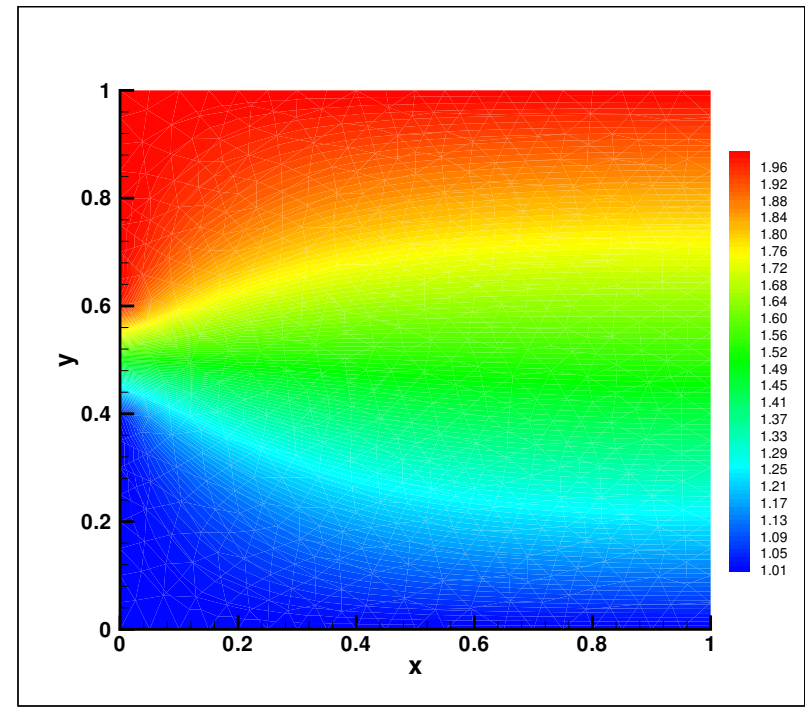

Figura 4.18: Gráfico de $\phi_{\mathrm{R}}$

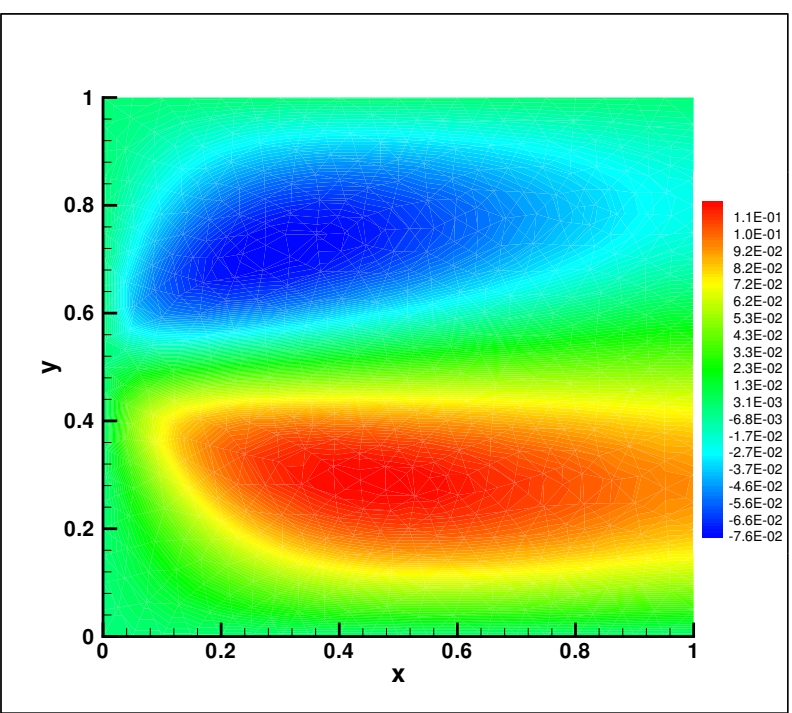

Figura 4.20: Termo fonte do dual inicial $\phi_{R}-\phi$

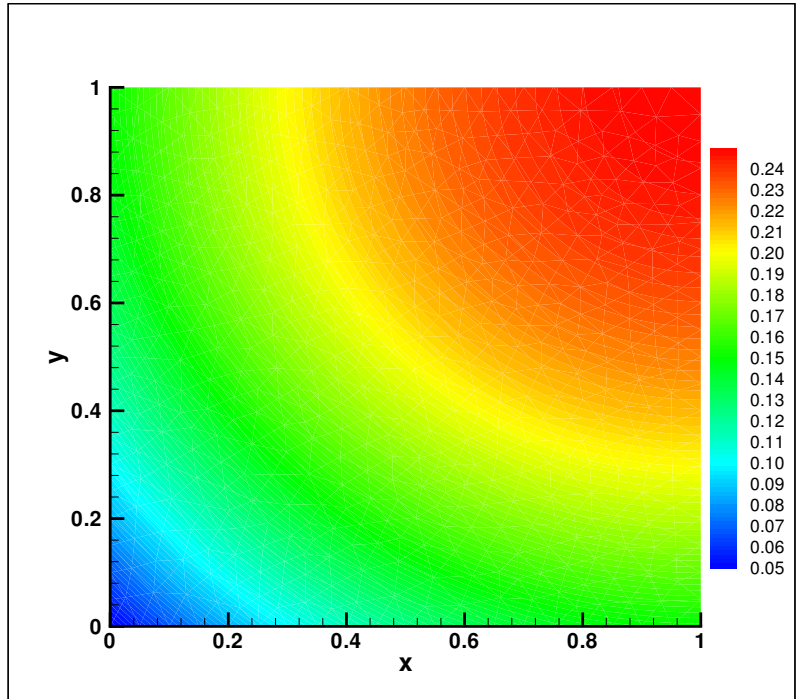

Figura 4.19: Função de referência

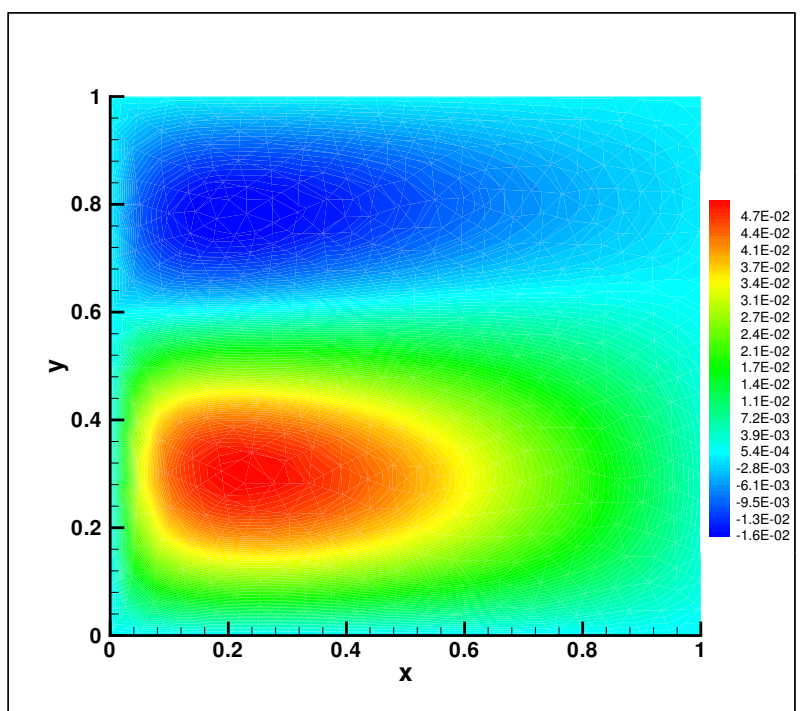

Figura 4.21: Gráfico da solução dual inicial $\psi$ 


\begin{tabular}{|c|c|l|}
\hline $1^{a}$ & it & iterações \\
\hline $2^{a}$ & $\mathcal{F}$ & valor do funcional \\
\hline $3^{a}$ & $\delta_{a} \mathcal{F}$ & gradiente em relação a $a$ \\
\hline $4^{a}$ & $\delta_{b} \mathcal{F}$ & gradiente em relação a $b$ \\
\hline $5^{a}$ & $\delta_{c} \mathcal{F}$ & gradiente em relação a $c$ \\
\hline $6^{a}$ & $\delta_{d} \mathcal{F}$ & gradiente em relação a $d$ \\
\hline $7^{a}$ & $\|\delta \mathcal{F}\|$ & norma 2 do gradiente \\
\hline $8^{a}$ & $\mathrm{CP}$ & número de chamadas do primal \\
\hline $9^{a}$ & $\mathrm{t}(\mathrm{seg})$ & tempo gasto na busca unidirecional (em segundos) \\
\hline $10^{a}$ & $\mathrm{TCG}$ & tempo de cálculo do gradiente (em segundos) \\
\hline $11^{a}$ & $\mathrm{a}$ & estimativa do primeiro parâmetro \\
\hline $12^{a}$ & $\mathrm{~b}$ & estimativa do segundo parâmetro \\
\hline $13^{a}$ & $\mathrm{c}$ & estimativa do terceiro parâmetro \\
\hline $14^{a}$ & $\mathrm{~d}$ & estimativa do quarto parâmetro \\
\hline
\end{tabular}

Tabela 4.28: Teste 2: Descrição das tabelas

o que pode ser verificado analisando $k(x, y)=a x+b y+c x(1-x)+d y(1-y)+C_{0}$. Assim sendo, deve-se ter na convergência que $a+c=0.2$ e $b+d=0.2$.

O comportamento dos métodos de busca, nesse caso de quatro parâmetros, tanto usando método adjunto como diferença finita, teve comportamento análogo ao que ocorreu no caso anterior de estimativa de três parâmetros. Esses resultados podem ser vistos nas tabelas a seguir.

\begin{tabular}{|c|c|c|c|c|c|c|c|c|c|c|c|c|c|}
\hline$\overline{\text { it }}$ & $\overline{\mathcal{F}}$ & $\overline{\overline{\delta_{a} \mathcal{F}}}$ & $\overline{\delta_{b} \mathcal{F}}$ & $\overline{\delta_{c} \mathcal{F}}$ & $\overline{\delta_{d} \mathcal{F}}$ & $\mid \overline{\delta \mathcal{F} \mid}$ & $\overline{C P}$ & t(seg) & TCG & $\bar{a}$ & $\bar{b}$ & $\mathrm{c}$ & $\bar{d}$ \\
\hline 0 & $1.9 \mathrm{e}-03$ & $2.3 e-02$ & $5.2 \mathrm{e}-02$ & $1.4 \mathrm{e}-02$ & $3.4 \mathrm{e}-02$ & $6.8 \mathrm{e}-02$ & 1 & 23 & 40 & 0.000 & 0.000 & 0.000 & 0.000 \\
\hline 1 & $3.4 \mathrm{e}-05$ & $9.9 \mathrm{e}-04$ & $-1.2 \mathrm{e}-03$ & $3.4 \mathrm{e}-04$ & $5.4 \mathrm{e}-06$ & $1.6 \mathrm{e}-03$ & 14 & 169 & 30 & 0.051 & 0.116 & 0.030 & 0.076 \\
\hline 2 & $2.1 \mathrm{e}-05$ & $1.3 e-03$ & $7.4 \mathrm{e}-05$ & $5.8 e-04$ & $5.9 e-04$ & $1.6 e-03$ & 21 & 192 & 19 & 0.067 & 0.097 & 0.036 & 0.076 \\
\hline 3 & $1.5 \mathrm{e}-07$ & $-1.9 e-05$ & $9.0 \mathrm{e}-05$ & $3.0 \mathrm{e}-06$ & 3.0e-05 & $9.7 e-05$ & 21 & 135 & 14 & 0.123 & 0.100 & 0.060 & 0.100 \\
\hline
\end{tabular}

Tabela 4.29: Malha 1: MGC com MRAO de ordem 3 usando método adjunto - 4 parâmetros

\begin{tabular}{|c|c|c|c|c|c|c|c|c|c|c|c|c|c|}
\hline it & $\mathcal{F}$ & $\delta_{a} \mathcal{F}$ & $\delta_{b} \mathcal{F}$ & $\delta_{c} \mathcal{F}$ & $\delta_{d} \mathcal{F}$ & $\|\delta \mathcal{F}\|$ & $\mathrm{CP}$ & $t(\operatorname{seg})$ & TCG & $a$ & $\mathrm{~b}$ & c & $\mathrm{d}$ \\
\hline 0 & $1.9 \mathrm{e}-03$ & $2.0 \mathrm{e}-02$ & $3.4 \mathrm{e}-02$ & $1.1 \mathrm{e}-02$ & $1.6 \mathrm{e}-02$ & $4.4 e-02$ & 1 & 23 & 75 & 0.000 & 0.000 & 0.000 & 0.000 \\
\hline 1 & $3.0 e-05$ & $5.4 \mathrm{e}-04$ & $-1.0 \mathrm{e}-03$ & $2.6 \mathrm{e}-04$ & $3.6 \mathrm{e}-04$ & $1.3 e-03$ & 15 & 162 & 45 & 0.070 & 0.118 & 0.039 & 0.058 \\
\hline 2 & $1.7 \mathrm{e}-05$ & $5.2 \mathrm{e}-04$ & $-2.3 e-04$ & $3.1 \mathrm{e}-04$ & $5.1 \mathrm{e}-04$ & $8.3 e-04$ & 21 & 195 & 44 & 0.080 & 0.100 & 0.044 & 0.064 \\
\hline 3 & $6.8 \mathrm{e}-06$ & $4.7 e-04$ & $3.6 e-04$ & $3.2 \mathrm{e}-04$ & $5.7 e-04$ & $8.8 e-04$ & 22 & 167 & 41 & 0.108 & 0.088 & 0.060 & 0.092 \\
\hline 4 & 5.6e-07 & $-2.5 e-05$ & $-1.1 \mathrm{e}-04$ & $9.9 \mathrm{e}-07$ & $-7.2 e-06$ & $1.2 \mathrm{e}-04$ & 22 & 158 & 37 & 0.123 & 0.099 & 0.070 & 0.110 \\
\hline
\end{tabular}

Tabela 4.30: Malha 1: MGC com MRAO de ordem 3 usando diferenças finitas - 4 parâmetros 


\begin{tabular}{|c|c|c|c|c|c|c|c|c|c|c|c|c|c|}
\hline it & $\overline{\mathcal{F}}$ & $\overline{\overline{\delta_{a} \mathcal{F}}}$ & $\overline{\delta_{b} \mathcal{F}}$ & $\overline{\delta_{c} \mathcal{F}}$ & $\overline{\delta_{d} \mathcal{F}}$ & $\overline{\|\delta \mathcal{F}\|}$ & $\overline{C P}$ & t(seg) & $\overline{\overline{T C G}}$ & $\bar{a}$ & $\bar{b}$ & c & $\bar{d}$ \\
\hline $\bar{~} 1$ & $7.6 \mathrm{e}-04$ & $1.3 e-02$ & $6.3 e-03$ & $6.4 \mathrm{e}-03$ & $1.4 \mathrm{e}-02$ & $2.1 \mathrm{e}-02$ & 1 & 95 & $\bar{~} 164$ & 0.000 & 0.000 & 0.000 & 0.000 \\
\hline 1 & $3.2 \mathrm{e}-05$ & 8.3e-05 & $2.3 e-03$ & $4.2 \mathrm{e}-05$ & $1.9 \mathrm{e}-04$ & $2.3 e-03$ & 16 & 519 & 86 & 0.162 & 0.076 & 0.077 & 0.166 \\
\hline 2 & $1.7 e-05$ & $1.0 \mathrm{e}-04$ & $1.5 \mathrm{e}-03$ & 4.0e-05 & $-8.6 e-06$ & $1.5 e-03$ & 19 & 600 & 78 & 0.163 & 0.100 & 0.077 & 0.168 \\
\hline 3 & $1.7 \mathrm{e}-05$ & $1.9 \mathrm{e}-04$ & $2.1 \mathrm{e}-03$ & $7.0 \mathrm{e}-05$ & $-5.8 e-05$ & $2.1 \mathrm{e}-03$ & 20 & 566 & 42 & 0.163 & 0.102 & 0.077 & 0.168 \\
\hline 4 & $1.7 \mathrm{e}-05$ & $2.8 e-04$ & $2.8 \mathrm{e}-03$ & $1.1 \mathrm{e}-04$ & $-1.1 \mathrm{e}-04$ & $2.8 \mathrm{e}-03$ & 19 & 530 & 34 & 0.164 & 0.103 & 0.077 & 0.168 \\
\hline 5 & $1.7 e-05$ & $3.8 e-04$ & $3.4 \mathrm{e}-03$ & $1.4 \mathrm{e}-04$ & $-1.5 e-04$ & $3.4 \mathrm{e}-03$ & 19 & 526 & 55 & 0.164 & 0.103 & 0.077 & 0.168 \\
\hline 6 & $1.7 e-05$ & $5.1 \mathrm{e}-04$ & 4.3e-03 & $1.9 \mathrm{e}-04$ & $-2.0 e-04$ & 4.3e-03 & 18 & 501 & 26 & 0.164 & 0.103 & 0.077 & 0.168 \\
\hline 7 & $1.7 e-05$ & $6.5 e-04$ & $5.2 \mathrm{e}-03$ & $2.4 \mathrm{e}-04$ & $-2.6 e-04$ & $5.2 \mathrm{e}-03$ & 18 & 504 & 27 & 0.164 & 0.103 & 0.077 & 0.168 \\
\hline 8 & $1.7 e-05$ & $7.4 \mathrm{e}-04$ & $5.8 \mathrm{e}-03$ & $2.7 e-04$ & $-3.0 e-04$ & $5.8 e-03$ & 17 & 477 & 49 & 0.164 & 0.103 & 0.077 & 0.168 \\
\hline 9 & $1.7 e-05$ & $8.8 e-04$ & $6.7 \mathrm{e}-03$ & $3.3 e-04$ & $-3.6 e-04$ & $6.8 e-03$ & 17 & 478 & 26 & 0.164 & 0.103 & 0.077 & 0.168 \\
\hline 10 & $1.7 e-05$ & $1.0 \mathrm{e}-03$ & $7.7 e-03$ & $3.8 \mathrm{e}-04$ & $-4.2 \mathrm{e}-04$ & $7.8 \mathrm{e}-03$ & 17 & 477 & 26 & 0.164 & 0.103 & 0.077 & 0.168 \\
\hline 11 & $1.7 \mathrm{e}-05$ & $1.2 \mathrm{e}-03$ & $8.6 e-03$ & $4.3 e-04$ & $-4.7 e-04$ & $8.7 e-03$ & 16 & 460 & 29 & 0.164 & 0.103 & 0.077 & 0.168 \\
\hline 12 & $1.7 e-05$ & $1.2 \mathrm{e}-03$ & $8.8 \mathrm{e}-03$ & $4.5 e-04$ & $-5.0 e-04$ & $8.9 e-03$ & 16 & 455 & 47 & 0.164 & 0.103 & 0.077 & 0.168 \\
\hline 13 & $1.7 \mathrm{e}-05$ & $1.4 \mathrm{e}-03$ & $9.8 \mathrm{e}-03$ & $5.0 \mathrm{e}-04$ & $-5.6 e-04$ & $9.9 e-03$ & 16 & 453 & 27 & 0.164 & 0.103 & 0.077 & 0.168 \\
\hline 14 & $1.7 e-05$ & $1.5 e-03$ & $1.1 \mathrm{e}-02$ & $5.6 e-04$ & $-6.2 e-04$ & $1.1 \mathrm{e}-02$ & 16 & 452 & 26 & 0.164 & 0.104 & 0.077 & 0.168 \\
\hline 15 & $1.7 e-05$ & $1.7 e-03$ & $1.2 \mathrm{e}-02$ & $6.1 \mathrm{e}-04$ & $-6.8 e-04$ & $1.2 \mathrm{e}-02$ & 16 & 476 & 27 & 0.164 & 0.104 & 0.077 & 0.168 \\
\hline 16 & $1.7 \mathrm{e}-05$ & $1.7 \mathrm{e}-03$ & $1.2 \mathrm{e}-02$ & $6.1 \mathrm{e}-04$ & $-6.8 e-04$ & $1.2 \mathrm{e}-02$ & 16 & 460 & 43 & 0.164 & 0.104 & 0.077 & 0.168 \\
\hline 17 & $1.7 e-05$ & $1.8 \mathrm{e}-03$ & $1.3 e-02$ & $6.6 e-04$ & $-7.5 e-04$ & $1.3 e-02$ & 16 & 449 & 26 & 0.164 & 0.104 & 0.077 & 0.168 \\
\hline 18 & $1.7 e-05$ & $2.0 \mathrm{e}-03$ & $1.4 \mathrm{e}-02$ & 7.3e-04 & $-8.2 \mathrm{e}-04$ & $1.4 \mathrm{e}-02$ & 15 & 440 & 25 & 0.164 & 0.104 & 0.077 & 0.168 \\
\hline 19 & $1.7 e-05$ & $2.0 \mathrm{e}-03$ & $1.4 \mathrm{e}-02$ & 7.3e-04 & $-8.4 e-04$ & $1.4 \mathrm{e}-02$ & 15 & 444 & 41 & 0.164 & 0.104 & 0.077 & 0.168 \\
\hline 20 & $1.7 \mathrm{e}-05$ & $2.1 \mathrm{e}-03$ & $1.5 \mathrm{e}-02$ & $7.7 \mathrm{e}-04$ & $-8.9 e-04$ & $1.5 \mathrm{e}-02$ & 15 & 426 & 25 & 0.164 & 0.104 & 0.077 & 0.168 \\
\hline
\end{tabular}

Tabela 4.31: Malha 1: MGC com MRAO de ordem 4 usando método adjunto - 4 parâmetros 


\begin{tabular}{|c|c|c|c|c|c|c|c|c|c|c|c|c|c|}
\hline it & $\overline{\mathcal{F}}$ & $\delta_{a} \mathcal{F}$ & $\overline{\delta_{b} \mathcal{F}}$ & $\overline{\delta_{c} \mathcal{F}}$ & $\overline{\delta_{d} \mathcal{F}}$ & $\mid \overline{\mid \delta \mathcal{F}}$ & $\mathrm{CP}$ & $t(\operatorname{seg})$ & TCG & $a$ & $\mathrm{~b}$ & $\mathrm{c}$ & $\bar{d}$ \\
\hline$\overline{0}$ & $1.8 \mathrm{e}-03$ & $1.9 \mathrm{e}-02$ & $3.1 \mathrm{e}-02$ & $1.1 \mathrm{e}-02$ & $1.5 \mathrm{e}-02$ & $4.1 \mathrm{e}-02$ & $\overline{1}$ & $\overline{122}$ & 397 & 0.000 & 0.000 & 0.000 & 0.000 \\
\hline 1 & $2.8 \mathrm{e}-05$ & $5.1 \mathrm{e}-04$ & $-1.0 e-03$ & $2.5 \mathrm{e}-04$ & $3.5 e-04$ & $1.2 \mathrm{e}-03$ & 15 & 875 & 243 & 0.072 & 0.117 & 0.040 & 0.056 \\
\hline 2 & $1.6 \mathrm{e}-05$ & $4.8 \mathrm{e}-04$ & $-2.0 e-04$ & $2.8 \mathrm{e}-04$ & $4.7 e-04$ & $7.5 e-04$ & 21 & 1055 & 243 & 0.081 & 0.099 & 0.044 & 0.062 \\
\hline 3 & $6.6 \mathrm{e}-06$ & 4.3e-04 & 3.3e-04 & $2.9 \mathrm{e}-04$ & $5.3 e-04$ & 8.1e-04 & 22 & 925 & 224 & 0.106 & 0.089 & 0.059 & 0.087 \\
\hline 4 & $2.0 \mathrm{e}-07$ & $1.5 e-05$ & $-1.4 e-04$ & $2.4 \mathrm{e}-05$ & $3.8 e-05$ & $1.5 \mathrm{e}-04$ & 22 & 894 & 263 & 0.121 & 0.100 & 0.069 & 0.105 \\
\hline
\end{tabular}

Tabela 4.32: Malha 1: MGC com MRAO de ordem 4 usando diferenças finitas - 4 parâmetros

\begin{tabular}{|c|c|c|c|c|c|c|c|c|c|c|c|c|c|}
\hline it & $\mathcal{F}$ & $\delta_{a} \mathcal{F}$ & $\delta_{b} \mathcal{F}$ & $\delta_{c} \mathcal{F}$ & $\delta_{d} \mathcal{F}$ & $\overline{|\delta \mathcal{F}| \mid}$ & $\mathrm{CP}$ & $t$ (seg) & $\overline{\mathrm{TCG}}$ & a & $\bar{b}$ & c & $\bar{d}$ \\
\hline 0 & $1.9 \mathrm{e}-03$ & $2.3 e-02$ & $4.2 \mathrm{e}-02$ & $1.3 e-02$ & $2.9 \mathrm{e}-02$ & $5.7 e-02$ & 1 & 86 & 145 & 0.000 & 0.000 & 0.000 & 0.000 \\
\hline 1 & $2.5 e-05$ & $7.0 \mathrm{e}-04$ & $-9.6 e-04$ & $2.6 e-04$ & $1.2 \mathrm{e}-04$ & $1.2 \mathrm{e}-03$ & 14 & 475 & 67 & 0.062 & 0.114 & 0.035 & 0.078 \\
\hline 2 & $1.5 \mathrm{e}-05$ & $7.4 \mathrm{e}-04$ & $3.6 e-05$ & $3.5 e-04$ & $4.7 e-04$ & $9.5 e-04$ & 21 & 451 & 45 & 0.074 & 0.097 & 0.039 & 0.080 \\
\hline 3 & $1.3 e-07$ & $-1.1 \mathrm{e}-05$ & $3.0 \mathrm{e}-05$ & $2.9 \mathrm{e}-06$ & 8.7e-06 & 3.3e-05 & 22 & 260 & 27 & 0.117 & 0.099 & 0.060 & 0.107 \\
\hline
\end{tabular}

Tabela 4.33: Malha 2: MGC com MRAO de ordem 3 usando método adjunto - 4 parâmetros

\begin{tabular}{|c|c|c|c|c|c|c|c|c|c|c|c|c|c|}
\hline it & $\mathcal{F}$ & $\delta_{a} \mathcal{F}$ & $\delta_{b} \mathcal{F}$ & $\delta_{c} \mathcal{F}$ & $\delta_{d} \mathcal{F}$ & $\|\delta \mathcal{F}\|$ & $\mathrm{CP}$ & $t(\operatorname{seg})$ & TCG & $a$ & $\mathrm{~b}$ & C & $\mathrm{d}$ \\
\hline 0 & $1.9 \mathrm{e}-03$ & $2.0 \mathrm{e}-02$ & $3.4 \mathrm{e}-02$ & $1.1 \mathrm{e}-02$ & $1.6 \mathrm{e}-02$ & $4.4 \mathrm{e}-02$ & 1 & 79 & 233 & 0.000 & 0.000 & 0.000 & 0.000 \\
\hline 1 & $2.9 \mathrm{e}-05$ & $5.3 e-04$ & $-9.9 e-04$ & $2.7 e-04$ & $3.7 \mathrm{e}-04$ & $1.2 \mathrm{e}-03$ & 14 & 426 & 98 & 0.071 & 0.118 & 0.039 & 0.058 \\
\hline 2 & $1.6 \mathrm{e}-05$ & $4.9 \mathrm{e}-04$ & $-1.6 e-04$ & $2.9 \mathrm{e}-04$ & $4.9 \mathrm{e}-04$ & $7.7 e-04$ & 21 & 407 & 94 & 0.081 & 0.099 & 0.044 & 0.065 \\
\hline 3 & $4.7 \mathrm{e}-06$ & $2.6 \mathrm{e}-04$ & $3.2 \mathrm{e}-04$ & $1.9 \mathrm{e}-04$ & $3.5 e-04$ & $5.7 e-04$ & 22 & 293 & 91 & 0.110 & 0.089 & 0.062 & 0.094 \\
\hline 4 & 8.3e-08 & $2.9 \mathrm{e}-05$ & $-1.2 \mathrm{e}-04$ & $3.3 e-05$ & $5.0 \mathrm{e}-05$ & $1.4 \mathrm{e}-04$ & 22 & 356 & 87 & 0.118 & 0.100 & 0.068 & 0.105 \\
\hline
\end{tabular}

Tabela 4.34: Malha 2: MGC com MRAO de ordem 3 usando diferenças finitas - 4 parâmetros

Novamente, perceba que o TCG para os métodos adjuntos em cada iteração é bem menor que o tempo gasto para diferenças finitas. Os testes mostraram que o método adjunto, para o caso das formas funcionais $\kappa(x, y)$, que são funções quadráticas, é sensível ao grau de refinamento da malha. A malha 1 é uma malha mais grossa que as malhas 2 e 3 . A medida que houve o refinamento das malha, o método adjunto começou a se comportar melhor atingindo o critério de convergência. Já diferenças finitas não mostrou ser muito sensível na malha 1 nos teste 2 e 3, tanto para reconstruções de ordem 3 como ordem 4.

Os 3 testes realizados mostraram a validade do método adjunto como ferramenta para estimar parâmetros. Em comparação diferenças finitas, o método adjunto em tem um custo computacional mais baixo para calcular os gradientes do que as técnicas baseadas em diferenças finitas. A diferença nesse custo entre as duas técnicas aumenta a medida que o número de parâmetros a serem estimados aumenta, com o método adjunto gastando bem menos. Tem-se ainda que parece existir uma relação entre a forma funcional do coeficiente de difusão e o 


\begin{tabular}{|c|c|c|c|c|c|c|c|c|c|c|c|c|c|}
\hline it & $\mathcal{F}$ & $\delta_{a} \mathcal{F}$ & $\delta_{b} \mathcal{F}$ & $\overline{\delta_{c} \mathcal{F}}$ & $\overline{\delta_{d} \mathcal{F}}$ & $\|\delta \mathcal{F}\|$ & $\mathrm{CP}$ & $\mathrm{t}(\mathrm{seg})$ & TCG & a & $\mathrm{b}$ & $\mathrm{c}$ & $\bar{d}$ \\
\hline 0 & $1.8 \mathrm{e}-03$ & $1.7 \mathrm{e}-02$ & $1.7 \mathrm{e}-02$ & $8.4 \mathrm{e}-03$ & $1.4 \mathrm{e}-02$ & $2.9 \mathrm{e}-02$ & 1 & 342 & 567 & 0.000 & 0.000 & 0.000 & 0.000 \\
\hline 1 & 3.0e-06 & 8.6e-05 & $-4.2 \mathrm{e}-04$ & 4.5e-05 & $5.5 e-05$ & $4.4 \mathrm{e}-04$ & 15 & 1576 & 139 & 0.107 & 0.108 & 0.053 & 0.087 \\
\hline 2 & $1.6 \mathrm{e}-06$ & $4.4 \mathrm{e}-05$ & $-1.1 \mathrm{e}-04$ & $3.2 \mathrm{e}-05$ & $7.1 \mathrm{e}-05$ & $1.4 \mathrm{e}-04$ & 23 & 1724 & 84 & 0.108 & 0.101 & 0.053 & 0.088 \\
\hline 3 & $1.5 \mathrm{e}-06$ & $4.8 e-05$ & $-5.3 e-05$ & $3.7 e-05$ & $9.2 \mathrm{e}-05$ & $1.2 \mathrm{e}-04$ & 25 & 1736 & 76 & 0.109 & 0.099 & 0.054 & 0.089 \\
\hline 4 & $1.4 \mathrm{e}-06$ & $1.1 \mathrm{e}-04$ & $-2.3 e-05$ & $9.1 \mathrm{e}-05$ & $2.4 \mathrm{e}-04$ & $2.8 \mathrm{e}-04$ & 26 & 1647 & 91 & 0.111 & 0.097 & 0.056 & 0.094 \\
\hline 5 & $7.6 e-07$ & $7.9 \mathrm{e}-04$ & $1.1 \mathrm{e}-04$ & $6.5 \mathrm{e}-04$ & $1.8 \mathrm{e}-03$ & 2.0e-03 & 24 & 1142 & 245 & 0.117 & 0.096 & 0.060 & 0.106 \\
\hline
\end{tabular}

Tabela 4.35: Malha 2: MGC com MRAO de ordem 4 usando método adjunto - 4 parâmetros

\begin{tabular}{|c|c|c|c|c|c|c|c|c|c|c|c|c|c|}
\hline it & $\mathcal{F}$ & $\delta_{a} \mathcal{F}$ & $\delta_{b} \mathcal{F}$ & $\delta_{c} \mathcal{F}$ & $\delta_{d} \mathcal{F}$ & $\|\delta \mathcal{F}\|$ & $\mathrm{CP}$ & $t(\operatorname{seg})$ & TCG & $a$ & $b$ & $\mathrm{C}$ & $\mathrm{d}$ \\
\hline 0 & $1.8 \mathrm{e}-03$ & $2.0 \mathrm{e}-02$ & $3.2 \mathrm{e}-02$ & $1.1 \mathrm{e}-02$ & $1.6 \mathrm{e}-02$ & $4.2 \mathrm{e}-02$ & 1 & 339 & 1600 & 0.000 & 0.000 & 0.000 & 0.000 \\
\hline 1 & $2.8 e-05$ & $5.1 \mathrm{e}-04$ & $-1.0 \mathrm{e}-03$ & $2.6 e-04$ & $3.5 e-04$ & $1.2 \mathrm{e}-03$ & 15 & 1967 & 405 & 0.072 & 0.118 & 0.040 & 0.057 \\
\hline 2 & $1.6 \mathrm{e}-05$ & $4.7 e-04$ & $-2.2 \mathrm{e}-04$ & $2.8 \mathrm{e}-04$ & $4.7 \mathrm{e}-04$ & $7.5 e-04$ & 21 & 1825 & 409 & 0.081 & 0.100 & 0.045 & 0.063 \\
\hline 3 & $6.7 \mathrm{e}-06$ & 4.6e-04 & $3.1 \mathrm{e}-04$ & $3.1 \mathrm{e}-04$ & $5.5 e-04$ & $8.4 \mathrm{e}-04$ & 22 & 1441 & 390 & 0.106 & 0.088 & 0.059 & 0.088 \\
\hline 4 & $3.1 \mathrm{e}-07$ & $-1.8 e-05$ & $-1.0 \mathrm{e}-04$ & $3.1 \mathrm{e}-06$ & $-2.7 e-07$ & $1.0 \mathrm{e}-04$ & 22 & 1400 & 366 & 0.121 & 0.098 & 0.069 & 0.106 \\
\hline
\end{tabular}

Tabela 4.36: Malha 2: MGC com MRAO de ordem 4 usando diferenças finitas - 4 parâmetros

\begin{tabular}{|c|c|c|c|c|c|c|c|c|c|c|c|c|c|}
\hline$\overline{\text { it }}$ & $\overline{\mathcal{F}}$ & $\overline{\delta_{a} \mathcal{F}}$ & $\overline{\overline{\delta_{b} \mathcal{F}}}$ & $\overline{\overline{\delta_{c} \mathcal{F}}}$ & $\overline{\delta_{d} \mathcal{F}}$ & $\mid \overline{\mid \delta \mathcal{F} \|}$ & $\overline{C \mathrm{CP}}$ & $\bar{t}$ t(seg) & $\overline{\overline{T C G}}$ & $\bar{a}$ & $\bar{b}$ & $\bar{c}$ & $\overline{\mathrm{d}}$ \\
\hline$\overline{\overline{0}}$ & $\overline{1.9 \mathrm{e}-03}$ & $2.2 \mathrm{e}-02$ & 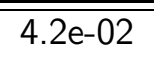 & 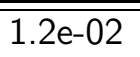 & $\overline{2.6 e-02}$ & $\overline{5.6 \mathrm{e}-02}$ & 1 & $\overline{195}$ & 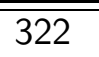 & 0.000 & 0.000 & 0.000 & 0.000 \\
\hline 1 & $3.1 \mathrm{e}-05$ & $7.3 e-04$ & $-9.6 e-04$ & $2.9 \mathrm{e}-04$ & $2.1 \mathrm{e}-04$ & $1.3 e-03$ & 14 & 912 & 147 & 0.061 & 0.117 & 0.034 & 0.071 \\
\hline 2 & $1.6 \mathrm{e}-05$ & $6.6 \mathrm{e}-04$ & $1.9 \mathrm{e}-05$ & 3.3e-04 & 4.7e-04 & 8.7e-04 & 21 & 875 & 96 & 0.075 & 0.098 & 0.040 & 0.075 \\
\hline 3 & $1.2 \mathrm{e}-07$ & $-3.4 e-06$ & $-1.9 e-05$ & $5.6 \mathrm{e}-06$ & $3.1 \mathrm{e}-06$ & $2.0 \mathrm{e}-05$ & 22 & 524 & 58 & 0.119 & 0.099 & 0.061 & 0.105 \\
\hline
\end{tabular}

Tabela 4.37: Malha 3: MGC com MRAO de ordem 3 usando método adjunto - 4 parâmetros

\begin{tabular}{|c|c|c|c|c|c|c|c|c|c|c|c|c|c|}
\hline it & $\mathcal{F}$ & $\delta_{a} \mathcal{F}$ & $\delta_{b} \mathcal{F}$ & $\delta_{c} \mathcal{F}$ & $\delta_{d} \mathcal{F}$ & $\overline{\|\delta \mathcal{F}\|}$ & $\mathrm{CP}$ & $t(\operatorname{seg})$ & TCG & $a$ & $\bar{b}$ & $\mathrm{C}$ & $\bar{d}$ \\
\hline 0 & $1.9 \mathrm{e}-03$ & $2.0 \mathrm{e}-02$ & $3.4 \mathrm{e}-02$ & $1.1 \mathrm{e}-02$ & $1.6 \mathrm{e}-02$ & $4.4 \mathrm{e}-02$ & 1 & 191 & 877 & 0.000 & 0.000 & 0.000 & 0.000 \\
\hline 1 & $2.9 \mathrm{e}-05$ & 5.3e-04 & $-9.4 e-04$ & $3.7 e-04$ & $4.5 e-04$ & $1.2 \mathrm{e}-03$ & 15 & 945 & 678 & 0.071 & 0.118 & 0.040 & 0.058 \\
\hline 2 & $1.4 \mathrm{e}-05$ & $4.5 e-04$ & $-1.0 \mathrm{e}-04$ & $3.2 \mathrm{e}-04$ & $5.0 \mathrm{e}-04$ & $7.6 \mathrm{e}-04$ & 21 & 870 & 543 & 0.082 & 0.098 & 0.047 & 0.067 \\
\hline 3 & $2.5 \mathrm{e}-06$ & $7.8 \mathrm{e}-05$ & $2.0 \mathrm{e}-04$ & 7.7e-05 & $1.4 \mathrm{e}-04$ & $2.7 e-04$ & 22 & 657 & 176 & 0.109 & 0.092 & 0.067 & 0.097 \\
\hline 4 & $4.0 \mathrm{e}-08$ & $7.9 \mathrm{e}-05$ & $-1.7 e-05$ & $8.7 e-05$ & $1.4 \mathrm{e}-04$ & $1.8 \mathrm{e}-04$ & 24 & 708 & 167 & 0.113 & 0.100 & 0.070 & 0.103 \\
\hline
\end{tabular}

Tabela 4.38: Malha 3: MGC com MRAO de ordem 3 usando diferenças finitas - 4 parâmetros

\begin{tabular}{|c|c|c|c|c|c|c|c|c|c|c|c|c|c|}
\hline it & $\overline{\mathcal{F}}$ & $\overline{\overline{\delta_{a} \mathcal{F}}}$ & $\overline{\delta_{b} \mathcal{F}}$ & $\overline{\delta_{c} \mathcal{F}}$ & $\overline{\delta_{d} \mathcal{F}}$ & $\overline{\|\delta \mathcal{F}\|}$ & $\overline{C P}$ & $t$ (seg) & TCG & $\bar{a}$ & $\bar{b}$ & $\bar{C}$ & $\overline{\mathrm{d}}$ \\
\hline 0 & $1.8 \mathrm{e}-03$ & $1.8 \mathrm{e}-02$ & $2.3 e-02$ & $8.8 \mathrm{e}-03$ & $1.3 e-02$ & $3.3 e-02$ & 1 & 975 & 1609 & 0.000 & 0.000 & 0.000 & 0.000 \\
\hline 1 & $1.5 \mathrm{e}-05$ & 3.0e-04 & $-6.1 e-04$ & $1.6 \mathrm{e}-04$ & $2.4 \mathrm{e}-04$ & $7.4 \mathrm{e}-04$ & 15 & 3169 & 323 & 0.086 & 0.112 & 0.042 & 0.065 \\
\hline 2 & $9.7 \mathrm{e}-06$ & $2.9 \mathrm{e}-04$ & $-2.9 e-04$ & $1.7 \mathrm{e}-04$ & 3.0e-04 & $5.4 \mathrm{e}-04$ & 22 & 3240 & 291 & 0.092 & 0.100 & 0.046 & 0.070 \\
\hline 3 & $8.3 e-06$ & $4.1 \mathrm{e}-04$ & $3.8 e-07$ & $2.6 \mathrm{e}-04$ & $5.2 \mathrm{e}-04$ & $7.1 \mathrm{e}-04$ & 23 & 2931 & 282 & 0.101 & 0.091 & 0.051 & 0.079 \\
\hline 4 & $2.6 \mathrm{e}-06$ & $3.6 e-04$ & $3.8 e-04$ & $2.4 \mathrm{e}-04$ & $5.1 \mathrm{e}-04$ & $7.7 \mathrm{e}-04$ & 22 & 2002 & 170 & 0.120 & 0.091 & 0.063 & 0.104 \\
\hline 5 & $8.4 e-07$ & $1.6 \mathrm{e}-04$ & $5.3 e-04$ & $1.2 \mathrm{e}-04$ & $2.9 \mathrm{e}-04$ & $6.3 e-04$ & 22 & 2564 & 150 & 0.125 & 0.097 & 0.067 & 0.111 \\
\hline
\end{tabular}

Tabela 4.39: Malha 3: MGC com MRAO de ordem 4 usando método adjunto - 4 parâmetros 


\begin{tabular}{|c|c|c|c|c|c|c|c|c|c|c|c|c|c|}
\hline it & $\mathcal{F}$ & $\delta_{a} \mathcal{F}$ & $\delta_{b} \mathcal{F}$ & $\delta_{c} \mathcal{F}$ & $\delta_{d} \mathcal{F}$ & $\overline{\|\delta \mathcal{F}\|}$ & $\mathrm{CP}$ & $\mathrm{t}(\mathrm{seg})$ & TCG & $\bar{a}$ & $\overline{\bar{b}}$ & $\overline{\mathrm{C}}$ & $\overline{\bar{d}}$ \\
\hline$\overline{0}$ & $1.8 \mathrm{e}-03$ & $2.0 \mathrm{e}-02$ & $3.3 e-02$ & $1.1 \mathrm{e}-02$ & $1.6 \mathrm{e}-02$ & $4.3 e-02$ & $\overline{1}$ & 755 & 2068 & 0.000 & 0.000 & 0.000 & 0.000 \\
\hline 1 & $2.9 \mathrm{e}-05$ & $5.2 \mathrm{e}-04$ & $-9.4 e-04$ & $2.6 \mathrm{e}-04$ & $3.7 e-04$ & $1.2 \mathrm{e}-03$ & 15 & 3647 & 801 & 0.071 & 0.118 & 0.040 & 0.057 \\
\hline 2 & $1.5 \mathrm{e}-05$ & $4.7 \mathrm{e}-04$ & $-7.4 e-05$ & $2.8 \mathrm{e}-04$ & $4.8 e-04$ & $7.3 e-04$ & 21 & 3376 & 808 & 0.083 & 0.098 & 0.045 & 0.065 \\
\hline 3 & $2.4 \mathrm{e}-06$ & $2.1 \mathrm{e}-05$ & $2.2 \mathrm{e}-04$ & $3.6 e-05$ & 7.5e-05 & $2.3 e-04$ & 22 & 2211 & 725 & 0.121 & 0.091 & 0.068 & 0.104 \\
\hline 4 & $3.1 \mathrm{e}-07$ & $-4.3 e-05$ & $-4.6 e-05$ & $-6.9 e-06$ & $-1.5 e-05$ & $6.6 \mathrm{e}-05$ & 24 & 3163 & 713 & 0.122 & 0.098 & 0.069 & 0.106 \\
\hline
\end{tabular}

Tabela 4.40: Malha 3: MGC com MRAO de ordem 4 usando diferenças finitas - 4 parâmetros

grau de refinamento da malha para que o método adjunto funcione bem, como pode ser observado nos testes 2 e 3, onde ocorre uma melhoria no desempenho do método adjunto em relação ao método de diferenças finitas nas malhas mais refinadas.

O próximo capítulo tem por finalidade fazer análise da sensibilidade a perturbações nas tolerâncias no critério de parada do problema primal, problema dual, busca unidirecional e funcional, sendo este último utilizado como critério de parada para a busca dos parâmetros. 


\section{Análise de sensibilidade do processo de identificação de parâmetros}

\subsection{Introdução}

O processo de estimação de parâmetros envolve a resolução numérica de duas equações diferenciais parciais, a saber, a equação do problema primal

$$
\nabla(\vec{\omega} \phi)-\nabla(\kappa \nabla \phi)=0
$$

e a equação adjunta (ou dual)

$$
-\nabla(\vec{\omega} \psi)-\nabla(\kappa \nabla \psi)=\phi_{\mathrm{R}}-\phi
$$

para o cálculo dos gradientes. Como pode ser visto, nesse trabalho, faz-se uso do método dos volumes finitos para resolver as equações do problema primal e adjunto, o qual emprega o método de reconstrução de alta ordem [30] para calcular os fluxos nas faces dos volumes de controle. O método de Runge-Kutta multiestágio é um método de evolução temporal que é empregado na busca da solução das referidas EDPs. No Runge-Kutta o critério de parada é a norma do resíduo. Além disso, tem-se ainda a avaliação do funcional em cada iteração do processo de busca para verificar se o critério de parada foi atingido, e também a busca do tamanho do passo que é realizado utilizando o método do segmento áureo, tendo o mesmo uma tolerância como critério de parada. Variar então as tolerâncias do primal, dual e da busca unidimensional, pode provocar alterações, por exemplo, em fatores como qualidade dos parâmetros estimados, tempo de execução do código, número de chamadas do problema primal na busca do tamanho do passo ao aplicar o método do segmento áureo. Esse capítulo tem por finalidade realizar um estudo sobre as sensibilidades das tolerâncias dos problemas primal, dual (ou adjunto) e da busca unidirecional.

O problema proposto para comparar os efeitos na variação de três tolerâncias, a saber, tolerâncias primal, 
dual (ou adjunto) e busca unidirecional, corresponde ao problema teste de dois parâmetros do capítulo anterior. Nesses problemas as tolerâncias aplicadas constam na tabela 5.1. O estudo da sensibilidade será feito variando-se uma tolerância por vez, mantendo-se as outras fixas. Fixada uma malha e uma ordem de reconstrução, cada uma dessas tolerâncias será modificada, sendo essas modificações apresentadas nas tabelas 5.5, 5.7 e 5.9. O critério de parada em todos os testes é o da tolerância do funcional, fixado em $10^{-6}$. As colunas das tabelas são descritas na tabela 5.2 , sendo $C_{0}=0.05$.

\begin{tabular}{lc}
\hline \hline Fator & Tolerância \\
\hline \hline Primal & $10^{-6}$ \\
Dual & $10^{-6}$ \\
Busca unidirecional & $10^{-2}$ \\
\hline \hline
\end{tabular}

Tabela 5.1: Fatores base

\begin{tabular}{|l|l|}
\hline $1^{a}$ & Teste: número do teste \\
\hline $2^{a}$ & Malha: tipo de malha que varia de 1 a 3 de acordo com a tabela 5.3. \\
\hline $3^{a}$ & Ordem: ordem do gradiente \\
\hline $4^{a}$ & Fator perturbado: primal, dual e busca unidirecional \\
\hline $5^{a}$ & a: primeiro parâmetro estimado da função $k(x, y)=a x+b y+C_{0}$ \\
\hline $6^{a}$ & b: segundo parâmetro estimado da função $k(x, y)=a x+b y+C_{0}$ \\
\hline $7^{a}$ & Iterações: número de iterações do processo de busca (em segundos) \\
\hline $8^{a}$ & Tempo: Tempo total gasto até atingir o critério de parada (tolerância do funcional) \\
\hline $9^{a}$ & $\mathcal{F}(\kappa):$ valor do funcional ao atingir o critério de parada. \\
\hline
\end{tabular}

Tabela 5.2: Descrição das tabelas para análise de sensibilidade

\begin{tabular}{ccc}
\hline \hline MALHA & VÉRTICES & TRIÂNGULOS \\
\hline \hline 1 & 145 & 248 \\
2 & 535 & 988 \\
3 & 2074 & 3986 \\
\hline \hline
\end{tabular}

Tabela 5.3: Parâmetros das malhas

Reiterando, foram feitas algumas observações importantes no capítulo anterior quanto a solução do problema primal e da solução do problema adjunto. Em toda discretização de EDPs existem os erros que provocam as chamadas difusões numéricas. O refinamento da malha é uma prática que tem por finalidade amenizar o efeito da difusão numérica, fato este que foi sentido nos resultados apresentados no capítulo 
anterior. Tanto é que melhorou a qualidade dos gradientes. Um outro fato importante é a questão do termo fonte do adjunto. Em conseqüência a solução do problema adjunto tende a zero, uma vez que as condições de três fronteiras são nulas e o vetor velocidade de advecção é $\vec{\omega}=(1,0)$ faz com que a solução nula da fronteira se espalhe domínio adentro. Note ainda que na equação adjunta, quando o termo fonte tende a zero, independente do quanto $\kappa$ esteja impreciso, a solução tende a zero. A difusão numérica portanto é um ponto que deve ser levado em consideração na análise de sensibilidades que serão apresentadas nas próximas seções.

\subsection{Estudo da sensibilidade do primal}

Na tabela 5.5 temos os resultados para variações da tolerância de convergência do problema primal no processo de identificação da difusão com dois parâmetros.

\begin{tabular}{ccccccccc}
\hline \hline Teste & Malha & Ordem & Primal & $\mathrm{a}$ & $\mathrm{b}$ & Iterações & Tempo(seg) & $\mathcal{F}[\kappa]$ \\
\hline \hline 1 & 1 & 3 & $\mathbf{1 e - 0 6}$ & 0.101 & 0.100 & 3 & 807 & $1.2 \mathrm{e}-08$ \\
\hline 2 & 1 & 3 & $\mathbf{1 e - 0 5}$ & 0.096 & 0.104 & 3 & 564 & $7.8 \mathrm{e}-07$ \\
3 & 1 & 3 & $\mathbf{1 e - 0 7}$ & 0.101 & 0.099 & 3 & 1792 & $1.4 \mathrm{e}-08$ \\
\hline \hline 4 & 2 & 3 & $\mathbf{1 e - 0 6}$ & 0.100 & 0.100 & 3 & 2070 & $6.3 \mathrm{e}-09$ \\
\hline \hline 5 & 2 & 3 & $\mathbf{1 e - 0 5}$ & 0.110 & 0.101 & 4 & 1964 & $5.5 \mathrm{e}-07$ \\
6 & 2 & 3 & $\mathbf{1 e - 0 7}$ & 0.099 & 0.100 & 3 & 4481 & $1.5 \mathrm{e}-09$ \\
\hline \hline 7 & 3 & 3 & $\mathbf{1 e - 0 6}$ & 0.106 & 0.097 & 3 & 4844 & $3.3 \mathrm{e}-07$ \\
\hline \hline 8 & 3 & 3 & $\mathbf{1 e - 0 5}$ & 0.115 & 0.092 & 3 & 2183 & $3.2 \mathrm{e}-07$ \\
9 & 3 & 3 & $\mathbf{1 e - 0 7}$ & 0.095 & 0.102 & 3 & 6687 & $2.4 \mathrm{e}-07$ \\
\hline \hline 10 & 1 & 4 & $\mathbf{1 e - 0 6}$ & 0.110 & 0.098 & 1 & 1202 & $4.8 \mathrm{e}-07$ \\
\hline \hline 11 & 1 & 4 & $\mathbf{1 e - 0 5}$ & 0.109 & 0.097 & 1 & 959 & $3.9 \mathrm{e}-07$ \\
12 & 1 & 4 & $\mathbf{1 e - 0 7}$ & 0.110 & 0.098 & 1 & 2598 & $4.5 \mathrm{e}-07$ \\
\hline \hline 13 & 2 & 4 & $\mathbf{1 e - 0 6}$ & 0.089 & 0.102 & 1 & 3478 & $6.3 \mathrm{e}-07$ \\
\hline \hline 14 & 2 & 4 & $\mathbf{1 e - 0 5}$ & 0.101 & 0.094 & 1 & 11455 & $8.4 \mathrm{e}-07$ \\
15 & 2 & 4 & $\mathbf{1 e - 0 7}$ & 0.088 & 0.102 & 1 & 6492 & $5.7 \mathrm{e}-07$ \\
\hline \hline 16 & 3 & 4 & $\mathbf{1 e - 0 6}$ & 0.106 & 0.095 & 8 & 39594 & $8.4 \mathrm{e}-07$ \\
\hline \hline 17 & 3 & 4 & $\mathbf{1 e - 0 5}$ & 0.227 & 0.039 & 20 & 23226 & $1.1 \mathrm{e}-04$ \\
\hline \hline & 3 & 4 & $\mathbf{1 e - 0 7}$ & 0.100 & 0.098 & 3 & 27522 & $1.2 \mathrm{e}-07$ \\
\hline \hline
\end{tabular}

Tabela 5.5: Tabela com a sensibilidade dos primais

Considerando os testes feitos com a malha 1, em terceira ordem, que na tabela 5.5 correspondem aos 
testes 1,2 e 3 , observa-se que ao degradar a precisão do primal (teste 2), percebe-se uma degradação da qualidade do funcional de cerca de uma ordem de grandeza em relação ao teste de referência 1 , entretanto existe uma redução no custo computacional refletida na queda do tempo necessária para atingir a convergência do funcional de $10^{-6}$. Quando aumentamos a precisão do primal em relação ao teste 1 não há alteração em relação ao nível de convergência do funcional, entretanto existe um aumento no tempo gasto devido a esse aumento de precisão. Em todos os três testes (1,2 e 3) a precisão de convergência do funcional foi atingida, mas percebe-se a necessidade de um nível mínimo para precisão do primal para se alcançar uma certa tolerância do funcional.

Mantida a terceira ordem e refinando-se a malha, observa-se efeito semelhante de redução do custo computacional com a degradação da precisão do primal com era de se esperar devido essenciallmente ao menor número de iterações necessárias para cada convergência do problema primal. O número de iterações do método dos gradients conjugados para esse conjunto de testes ( 1 a 9 ) é praticamente o mesmo.

Aumentando a ordem da reconstrução de terceira para quarta ordem observa-se que na maiora dos casos ocorre convergência do funcional em apenas uma iteração. Quando isso não ocorre, especificamente nos casos 14 e 17, a dificuldade reside no fato da tolerância do primal estar incompatível com o refinamento de

malha e a escolha de ordem. Já no teste 18 , a precisão $10^{-7}$ exigida como tolerância para o primal tornou-se compatível com a ordem e o grau de refinamento da malha, exigindo menos da metade do número de iterações do teste 16 .

\subsection{Estudo da sensibilidade do dual}

Na tabela 5.7 temos os resultados para variações da tolerância de convergência do problema dual no processo de identificação da difusão com dois parâmetros.

Analisando os testes de 1 a 9 percebe-se que não é possível trabalhar com a tolerância degradada do dual para a malha mais fina pois nesse caso não há convergência após 20 iterações do processo (teste 8). Para os outros testes os resultados são equivalentes demonstrando pouca sensibilidade da tolerância do problema dual. Comportamento idêntico é observado para o conjunto de testes de quarta ordem (de 10 a 18). Quando a precisão do dual é degradadada (teste 17) novamente não se alcança convergência.

Nos testes 3, 6 e 9, o aumento na precisão do dual não provoca melhoria em qualidade nas estimativas dos parâmetros relação aos testes base 1, 4 e 7. A mesma coisa se observa-se para reconstrução de quarta 


\begin{tabular}{ccccccccc}
\hline \hline Teste & Malha & Ordem & Dual & a & b & Iterações & Tempo(seg) & $\mathcal{F}[\kappa]$ \\
\hline \hline 1 & 1 & 3 & $\mathbf{1 e - 0 6}$ & 0.101 & 0.100 & 3 & 807 & $1.2 \mathrm{e}-08$ \\
\hline 2 & 1 & 3 & $\mathbf{1 e - 0 5}$ & 0.101 & 0.101 & 3 & 1165 & $8.7 \mathrm{e}-08$ \\
3 & 1 & 3 & $\mathbf{1 e - 0 7}$ & 0.102 & 0.096 & 3 & 1412 & $5.7 \mathrm{e}-07$ \\
\hline \hline 4 & 2 & 3 & $\mathbf{1 e - 0 6}$ & 0.100 & 0.100 & 3 & 2070 & $6.3 \mathrm{e}-09$ \\
\hline \hline 5 & 2 & 3 & $\mathbf{1 e - 0 5}$ & 0.100 & 0.098 & 2 & 1908 & $1.1 \mathrm{e}-07$ \\
6 & 2 & 3 & $\mathbf{1 e - 0 7}$ & 0.098 & 0.101 & 3 & 2775 & $5.6 \mathrm{e}-08$ \\
\hline \hline 7 & 3 & 3 & $\mathbf{1 e - 0 6}$ & 0.106 & 0.097 & 3 & 4844 & $3.3 \mathrm{e}-07$ \\
\hline \hline 8 & 3 & 3 & $\mathbf{1 e - 0 5}$ & 0.055 & 0.118 & 20 & 19129 & $1.8 \mathrm{e}-05$ \\
9 & 3 & 3 & $\mathbf{1 e - 0 7}$ & 0.097 & 0.103 & 3 & 4211 & $4.8 \mathrm{e}-07$ \\
\hline \hline 10 & 1 & 4 & $\mathbf{1 e - 0 6}$ & 0.110 & 0.098 & 1 & 1202 & $4.8 \mathrm{e}-07$ \\
\hline \hline 11 & 1 & 4 & $\mathbf{1 e - 0 5}$ & 0.113 & 0.096 & 1 & 1907 & $9.1 \mathrm{e}-07$ \\
12 & 1 & 4 & $\mathbf{1 e - 0 7}$ & 0.109 & 0.097 & 1 & 1712 & $4.4 \mathrm{e}-07$ \\
\hline \hline 13 & 2 & 4 & $\mathbf{1 e - 0 6}$ & 0.088 & 0.102 & 1 & 3478 & $6.3 \mathrm{e}-07$ \\
\hline \hline 14 & 2 & 4 & $\mathbf{1 e - 0 5}$ & 0.089 & 0.101 & 1 & 3613 & $4.6 \mathrm{e}-07$ \\
15 & 2 & 4 & $\mathbf{1 e - 0 7}$ & 0.088 & 0.102 & 1 & 4342 & $6.4 \mathrm{e}-07$ \\
\hline \hline 16 & 3 & 4 & $\mathbf{1 e - 0 6}$ & 0.106 & 0.095 & 8 & 39594 & $8.4 \mathrm{e}-07$ \\
\hline \hline 17 & 3 & 4 & $\mathbf{1 e - 0 5}$ & 0.076 & 0.107 & 3 & 76902 & $3.8 \mathrm{e}-06$ \\
\hline \hline & 3 & 4 & $\mathbf{1 e - 0 7}$ & 0.102 & 0.096 & 5 & 24345 & $3.2 \mathrm{e}-07$ \\
\hline \hline
\end{tabular}

Tabela 5.7: Tabela com a sensibilidade dos duais 
ordem nos testes 12 e 15 em relação aos testes base 13 e 10. Agora na malha mais fina e na ordem mais alta, comparando-se o teste 18 com o teste 16 , pode-se observar que o aumento da ordem de precisão do dual provocou uma melhoria significativa na qualidade dos parâmetros, gastando quase metade do número de iterações.

\subsection{Estudo da sensibilidade da busca unidimensional}

Na tabela 5.9 temos os resultados para variações da tolerância de convergência da busca unidimensional via método do segmento áureo no processo de identificação da difusão com dois parâmetros.

\begin{tabular}{ccccccccc}
\hline \hline Teste & Malha & Ordem & Busca & $\mathrm{a}$ & $\mathrm{b}$ & Iteraç̃os & Tempo(seg) & $\mathcal{F}[\kappa]$ \\
\hline \hline 1 & 1 & 3 & $\mathbf{0 . 0 1}$ & 0.101 & 0.100 & 3 & 807 & $1.2 \mathrm{e}-08$ \\
\hline \hline 2 & 1 & 3 & $\mathbf{0 . 0 0 5}$ & 0.100 & 0.099 & 3 & 1232 & $7.9 \mathrm{e}-09$ \\
3 & 1 & 3 & $\mathbf{0 . 0 2}$ & 0.102 & 0.097 & 3 & 945 & $2.4 \mathrm{e}-07$ \\
\hline \hline 4 & 2 & 3 & $\mathbf{0 . 0 1}$ & 0.100 & 0.100 & 3 & 2070 & $6.3 \mathrm{e}-09$ \\
\hline \hline 5 & 2 & 3 & $\mathbf{0 . 0 0 5}$ & 0.093 & 0.102 & 3 & 2537 & $4.1 \mathrm{e}-07$ \\
6 & 2 & 3 & $\mathbf{0 . 0 2}$ & 0.098 & 0.100 & 3 & 2222 & $4 \mathrm{e}-08$ \\
\hline \hline 7 & 3 & 3 & $\mathbf{0 . 0 1}$ & 0.106 & 0.097 & 3 & 4844 & $3.3 \mathrm{e}-07$ \\
\hline \hline 8 & 3 & 3 & $\mathbf{0 . 0 0 5}$ & 0.100 & 0.098 & 3 & 4894 & $8.2 \mathrm{e}-08$ \\
9 & 3 & 3 & $\mathbf{0 . 0 2}$ & 0.101 & 0.101 & 3 & 3676 & $9.8 \mathrm{e}-08$ \\
\hline \hline 10 & 1 & 4 & $\mathbf{0 . 0 1}$ & 0.110 & 0.098 & 1 & 1202 & $4.8 \mathrm{e}-07$ \\
\hline \hline 11 & 1 & 4 & $\mathbf{0 . 0 0 5}$ & 0.109 & 0.097 & 1 & 1374 & $4.8 \mathrm{e}-07$ \\
12 & 1 & 4 & $\mathbf{0 . 0 2}$ & 0.109 & 0.097 & 1 & 1493 & $4.8 \mathrm{e}-07$ \\
\hline \hline 13 & 2 & 4 & $\mathbf{0 . 0 1}$ & 0.089 & 0.102 & 1 & 3478 & $6.3 \mathrm{e}-07$ \\
\hline \hline 14 & 2 & 4 & $\mathbf{0 . 0 0 5}$ & 0.088 & 0.102 & 1 & 3361 & $6.3 \mathrm{e}-07$ \\
15 & 2 & 4 & $\mathbf{0 . 0 2}$ & 0.089 & 0.102 & 1 & 3537 & $6.4 \mathrm{e}-07$ \\
\hline \hline 16 & 3 & 4 & $\mathbf{0 . 0 1}$ & 0.106 & 0.095 & 8 & 39594 & $8.4 \mathrm{e}-07$ \\
\hline \hline 17 & 3 & 4 & $\mathbf{0 . 0 0 5}$ & 0.100 & 0.097 & 4 & 24585 & $1.9 \mathrm{e}-07$ \\
\hline \hline & 3 & 4 & $\mathbf{0 . 0 2}$ & 0.102 & 0.098 & 4 & 18410 & $7.7 \mathrm{e}-08$ \\
\hline \hline
\end{tabular}

Tabela 5.9: Tabela com a sensibilidade das buscas unidimensionais

O efeito da degradação da tolerância da busca unidirecional parece ser mais acentuado, adquirido uma tendência monotônica nas malhas mais finas. Percebe-se que nas malhas mais mais finas, tanto utilizando reconstrução de terceira como reconstrução de quarta ordem, a diminuição da tolerância aumenta a precisão dos parâmetros. Em geral não se obtém uma relação direta entre custo, precisão do funcional e tolerância 
da busca unidirecional para malhas mais grossas.

O resumo das conclusões sobre estes testes será realizado no próximo capítulo. 


\section{Conclusões}

O método adjunto juntamente com o método de reconstrução de alta ordem, o qual foi utilizado no método dos volumes finitos para calcular os fluxos na faces, e portanto para resolver os problemas primais e adjuntos, mostrou sua validade no que tange sua aplicação em problemas de identificação de parâmetros.

A análise dos resultados nos permite dizer que a tolerância do funcional define uma região de vizinhança da solução com um certo raio $r_{f}$ que se deseja atingir. A escolha de malha e ordem define uma região de raio $r_{\varepsilon}$ onde o efeito da difusão numérica impede que o cálculo do gradiente seja preciso o suficiente para que o processo de identificação de parâmetros, no caso da própria difusão física seja eficaz. No método adjunto, esse raio é proporcional a ordem e tamanho da malha, no caso dos métodos de diferenças finitas temos mais um parâmetro de influência que é o divisor da fórmula de diferenças. A independência do método adjunto em relação a esse divisor, e a sua estrutura de implementação semelhante ao problema primal fazem dele uma escolha adequada e eficaz para a solução desses problemas.

Analisando os capítulos 2, 4 e 5, podemos reunir algumas conclusões sobre os pontos importantes envolvidos no processo processo de identificação de parâmetros:

1. Método de reconstrução de alta ordem: O método de reconstrução de alta ordem apresentado nesse trabalho mostrou ser eficaz no processo de cálculo dos fluxos nas faces tanto para o problema primal como para o problema adjunto. É uma técnica versátil no sentido da forma como é construído, permitindo que o método de reconstrução seja implementando de forma que a estrutura do código seja preservada. Como comentado no capítulo 2, uma vez que a ordem de reconstrução esteja definida, gerada a estrutura de dados para essa ordem, o cálculo dos elementos das matrizes dos coeficientes consiste essencialmente em integração de polinômios. Tendo as matrizes de reconstrução para cada volume de controle, as mesmas se preservam durante todo o processo de resolução de EDPs quando o MVF que emprega tal técnica de reconstrução.

Perceba ainda que, desde que se utilize a mesma ordem de reconstrução, a matriz dos coeficientes 
utilizada na reconstrução da solução em cada volume de controle do interior é a mesma tanto para o problema primal como para o problema adjunto, não exigindo a necessidade de gerar matrizes diferentes. $\mathrm{Na}$ fronteira o que varia apenas são as linhas de restrição pois o problema primal e adjunto tem condições de fronteira diferentes, sendo que as demais linhas são as mesmas. Esses fatores facilitam a implementação computacional.

2. Precisão de convergência do funcional: Para os problemas testes propostos, verificou-se que para atingir uma certa precisão do funcional, é necessária sintonização entre o nível de malha e ordem. Para malhas médias, no caso malha 2, o aumento da ordem parece ser mais efetivo. Para malhas mais finas, no caso malha 3, o aumento de ordem deve ser acompanhado por um aumento de precisão geral que pode incorrer no aumento excessivo do custo computacional. Entretanto, cada aplicação deve ter seus requisitos de precisão em sintonia com o problema em questão.

3. Tolerância da busca unidirecional: Aparentemente não se observa uma tendência monotônica na tolerância da busca unidirecional. Nesse trabalho, a escolha do método do segmento áureo para ser aplicado na busca do tamanho do passo teve por base sua simplicidade, e mostrou não ser o mais eficaz para o problema. Esse é um fator importante pois define o número de soluções do primal que corresponde a maior parte do custo computacional total no processo de estimação de parâmetros.

4. Efeito da ordem: Quando o ponto de partida encontra-se distante da solução percebe-se, para os problemas testados, que o uso do método adjunto em terceira ordem tem uma aproximação inicial do gradiente até pior que diferenças finitas. Entretanto, para as aproximações seguintes, os gradientes são consistentes o suficiente para permanecer na busca da solução. O uso de quarta ordem permite uma estimativa melhor desse gradiente inicial, levando a convergência rápida. O efeito da precisão do primeiro gradiente pode ser observada nas figuras 4.9, 4.11 e 4.13 para o caso de dois parâmetros. Nos testes 2 e 3 do capítulo 4, nas malhas mais refinadas e ordem mais elevada, o mesmo ocorre.

5. Custo computacional para calcular os gradientes: Comparando o método adjunto com o método de diferenças finitas, o método adjunto mostrou ter um custo computacional menor que o diferenças finitas para calcular os gradientes em cada iteração do método de otimização. A diferença nesse custo cresce gradativamente a medida que aumenta o número de parâmetros a serem estimados, o que pode ser verificado nas tabelas analisadas no capítulo 4, tendo o adjunto um desempenho melhor. 
6. Alta difusão: Em problemas onde o nível médio ou máximo de difusão é alto, o método adjunto sofre da mesma dificuldade de convergência do método de diferenças finitas, para os casos testados. Entretanto, mostra-se que com o refino da malha (geral ou adaptativo) o efeito do método adjunto se reestabeleça.

7. Difusão não-linear: Dependendo da forma funcional do coeficiente de difusão, o método adjunto mostrou ser sensível ao grau de refinamento da malha. Isso pode ser observado nos testes 2 e 3 do capítulo 4, cujas formas funcionais do coeficientes são funções quadráticas. Em malhas mais refinadas o método adjunto se comportou melhor, atingindo a convergência. Já o método de diferenças finitas não mostrou ser muito sensível para as referidas formas funcionais do coeficiente de difusão, embora tenha um custo computacional mais elevado do que o método adjunto.

8. Maior número de coeficientes $\mathbf{X}$ difusão não-linear: Para os casos testados, acredita-se que os resultados foram mais influenciados pela difusão mais alta, em relação ao caso linear, do que pelo número de coeficiente em si. Entretanto, observa-se a tendência compensador do uso do método adjunto em malhas mais refinadas.

Como propostas futuras de melhorias:

- O método Runke-Kutta multiestágio, utilizado nesse trabalho, é um método de evolução temporal que possui condições restritivas de estabilidade. Assim sendo, uma proposta futura consiste em procurar um método de resolução de EDPs baseado em MVF com condições menos restritivas de estabilidade e que tenha uma boa taxa de convergência como por exemplo, o GMRES matrix-free [46, 43, 48]. O GMRES matrix-free, nos artigos citados, surge no contexto onde a EDP é resolvida implicitamente utilizando o MVF em malha não-estruturada de triângulos. A matriz dos coeficientes envolvida no sistema linear que surge é esparsa e não-simétrica. Em essência, o GMRES matrix-free é uma técnica de resolução numérica do referido tipo de sistema linear sem que haja necessidade de montar a matriz dos coeficientes. As características do GMRES matrix-free servem então como motivação para propostas futuras no que tange ao método de resolução de EDPs.

- Busca do tamanho do passo: O método do segmento áureo mostrou-se um método lento para busca do tamanho do passo. Orlande [49] descreve em seu livro uma técnica de obtenção do tamanho do passo a ser explorada como proposta futura. 
- Com relação ao problema da fronteira de Neumann para reconstruções de segunda e quarta ordens, Gooch [47, 43], apresenta novas técnicas no que tange ao método de reconstrução de alta ordem. As técnicas apresentadas nessas referências podem ser exploradas em trabalhos futuros.

- Estudar os efeitos que podem ser provocados fazendo-se uso de ordens de reconstrução diferentes para o problema primal e para o adjunto em problemas de estimação de parâmetros. 


\section{Referências Bibliográficas}

[1] O. M. Alifanov. Inverse Heat Transfer Problems. Springer-Verlag, New York, 1994.

[2] M.V. Altena. High-Order Finite Volume Discretisations for Solving a Modified Advection-Diffusion Problem on Unstructured Triangular Meshes. PhD thesis, University of Waterloo, 1999.

[3] W.K. Anderson and V. Venkatakrishnan. Aerodynamic design optimization on unstructured grids with a continuous adjoint formulation. Computers \& Fluids, 1999.

[4] W.K. Anderson and V. Venkatakrishnan. Aerodynamic design optimization on unstructured grids with a continuous adjoint formulation. Computers and Fluids, pages 443-480, 1999.

[5] T. J. Barth. Recent improvements in high order k-exact reconstruction on unstructured meshes. $A I A A$ 93-0668, 1993.

[6] T.J. Barth and P.O. Frederickson. Higher order solution of the euler equations on unstructured grids using quadratic reconstruction. jan 8-11 1990.

[7] M.J. Colaço, H.R.B. Orlande, and G.S. Dulikravich. Inverse and optimization problems in heat transfer. Journal of the Brazilian Society of Mechanical, Sciences and Engineering, 2006.

[8] D.L. Darmofal and D A. Venditti. A multilevel error estimation and grid adaptative strategy for improving the accuracy of integral outputs. Technical report, Massachusetts Institute of Technology, 1999.

[9] D.L. Darmofal and D A. Venditti. Adjoint error estimation and grid adaptation for functional outputs: Application to quasi-one-dimensional flow. Journal of Computational Physics, 164:204-227, 2000.

[10] D.L. Darmofal and D A. Venditti. Grid adaptation for functional outputs: Application to two-dimensional inviscid flows. Journal of Computational Physics, 176:40-69, 2002. 
[11] D.L. Darmofal and D.A. Venditti. Anisotropic grid adaptation for functional output: Application to two-dimensional viscous flows. Journal of Computational Physics, 187:22-46, 2003.

[12] D. A. Dunavant. High degree efficient symmetrical gaussian quadrature rules for the triangle. International Journal for Numerical Methods in Engineering, 21:1129-1148, 1985.

[13] J.K. Elliot. Aerodynamic Optimization Based on the Euler and Navier-Stokes Equations Using Unstructured Grids. PhD thesis, Massachusetts Institute of Technology, 1998.

[14] M. Giles. On the use of runge-kutta time-marching and multigrid for the solution of steady adjoint equations. In AD2000 Conference in Nice, Oxford, June 19-23 2000.

[15] M. Giles and N. Pierce. Adjoint equations in cfd: Duality, boundary conditions and solution behavior. AIAA 97-1850, 1997.

[16] M. Giles and N. Pierce. Adjoint error correction for integral outputs. Technical report, Oxford University, 1998.

[17] M. Giles and N. Pierce. Adjoint recovery of superconvergent functionals from approximate solutions of partial differential equations. Technical report, Oxford University, 1998.

[18] M. Giles and N. Pierce. Improved lift and drag estimates using euler equations. Technical report, Oxford University, 1998.

[19] M. Giles and N. Pierce. On the properties of solutions of the adjoint euler equations. In 6th ICFD Conference on Numerical Methods for Fluid Dynamics, Oxford, 1998.

[20] M. Giles and N. Pierce. Adjoint recovery of superconvergent functionals from pde approximations. SIAM Review, 2000.

[21] M. Giles and N. Pierce. An introduction to the adjoint approach to design. Flow, Turbulence and Combustion, 65:393-415, 2000.

[22] M. Giles and N. Pierce. Analysis of adjoint error correction for superconvergent functional estimates. Technical report, 2001. 
[23] M. Giles and N. Pierce. Analytic adjoint solutions for the quasi-one-dimensional euler equations. Journal of Fluid Mechanics, 426:327-345, 2001.

[24] M. Giles and N. Pierce. Discrete adjoint approximations with shocks. Technical report, Oxford University, 2003.

[25] M. Giles, N. Pierce, M. C. Duta, and J.D. Müller. Algorithm developments for discrete adjoint methods. Technical report, 2003.

[26] G.H. Golub and C.F. Van Loan. Matrix Computation. The Johns Hopkins University Press, Baltimore, 1996.

[27] C.O. Gooch. Quasi-eno schemes for unstructured meshes based on unlimited data-dependent leastsquares reconstruction. Journal of Computational Physics, 133 (1):6-17, 1997.

[28] C.O. Gooch. Multigrid acceleration of an upwind euler solver on unstructured meshes. AIAA, 2002.

[29] C.O. Gooch. A toolkit for numerical simulation of pde's: I - fundamentals of generic finite-volume simulation. Computer Methods in Applied Mechanics and Engineering, 192 (9-10):1147-1175, 2003.

[30] C.O. Gooch and M.V. Altena. A high-order-accurate unstructured mesh finite-volume scheme for the advection-diffusion equation. Journal of Computational Physics, 181:729-752, 2002.

[31] A. Harten, S. Osher, and S.R. Chakravarthy. Some results on uniformly high-order accurate essentially nonoscillatory schemes. Applied Numerical Mathematics, 2:347-376, 1986.

[32] M.T. Heath. Scientific Computing - An Introductory Survey. McGraw-Hill, Illinois, 1997.

[33] A. Jameson. Aerodynamic design via control theory. Journal of Scientific Computing, 3:233-260, 1988.

[34] A. Jameson. Optimum aerodynamic design using cfd and control theory. AIAA, 1995.

[35] A. Jameson. Optimum aerodynamic design using control theory. Computational Fluid Dynamics Fluid Review, pages 495-528, 1995.

[36] A. Jameson. Aerodynamic shape optimization techniques based on control theory. AIAA 98 2538, 1998. 
[37] A. Jameson. Re-engineering the design process through computational. Journal Aircraft, 36:36-50, 1999.

[38] A. Jameson. Aerodynamic shape optimization using the adjoint method. Lectures at Von Karman Institute - Brussels, 2003.

[39] A. Jameson and S.K. Nadarajah. A comparation of the continuous and discrete adjoint approach to automatic aerodynamic optimization. In AIAA 38th Aerospace Sciences Meeting and Exhibit, Reno,NV, 2000.

[40] Y. Jarny, M. N. Özisik, and J. P. Bardon. A general optimization method using adjoint equation for solving multidimensional inverse heat conduction. International Journal Heat and Mass Transfer, pages 2911-2919, 1991.

[41] Dana Rae Lindquist. A Comparison of Numerical Schemes on Triangular and Qaudrilateral Meshes. PhD thesis, Massachusetts Institute of Technology, 1988.

[42] John Layer Lions. Optimal Control of Systems Governed by Partial Differential Equations. SpringerVerlag, New York, 1971.

[43] K. Michalak and C.O. Gooch. Matrix-explicit gmres for a higher-order accurate inviscid compressible flow solver. In Proceedings of 18th AIAA Computational Fluid Dynamics Conference. AIAA, 2007.

[44] B. Mohammadi. Fluid Dynamics Computation with NSC2KE. Inria, France, 1994.

[45] S. Nadarajah. The Discrete Adjoint Approach to Aerodynamic Shape Optimization. PhD thesis, Stanford University, 2003.

[46] A. Nejat and C.O.Gooch. A high-order accurate unstructured gmres solver for poisson's equation. In Eleventh Annual Conference of the Computational Fluid Dynamics Society of Canada, pages 344-349. CFD Society of Canada, 2003.

[47] A. Nejat, C.O.Gooch, and K. Michalak. On obtaining high-order finite-volume solutions to the euler equations on unstructured meshes. In Proceedings of 18th AIAA Computational Fluid Dynamics Conference. AIAA, june 25-28 2007. 
[48] A. Nejat and C.O. Gooch. On preconditioning of newton-gmres algorithm for a higher-order accurate unstructured solver. In Proceedings of the Fourteenth Annual Conference of the Computational Fluid Dynamics Society of Canada, 2006.

[49] H.R.B. Orlande and M.N. Özisik. Inverse Heat Transfer - Fundamentals and Applications. Taylor and Francis, New York, 2000.

[50] Kevin Parrot, Chris Aldridge, and Paul Nesvadba. Identification of the thermophysical properties of fish european study group with industry. Technical report, University of Greenwich, 1997.

[51] O. Pironneau. On optimum design in fluid mechanics. Journal of Fluid. Mech., 64:97-110, 1974.

[52] A. Quarteroni, R. Sacco, and F. Saleri. Numerical Mathematics. Springer-Verlag, New York, 2000.

[53] A. Rösch. Identification of nonlinear heat transfer laws by means of boundary data. Technical report, Technical University of Chemnitz Zwickau, 1999.

[54] E. Saltel and F. Hecht. EMC2 Wysiwyg 2D Finite Elements Mesh Generator. INRIA, www.inria.fr, out 1995.

[55] N. Zabaras. Adjoint methods for inverse free convection problems with application to solidification process. Adjoint Techniques for Inverse Free Convection Problems, 2000. 